\title{
CHARACTERIZATION OF ADHESIVELY BONDED JOINTS USING ACOUSTO-ULTRASONICS
}

by

\section{SAMSIR TANARY}

A thesis submitted to the Faculty of Graduate

Studies and Research in partial fulfillment

of the requirement for the degree of Master of Applied Science

Department of Mechanical Engineering

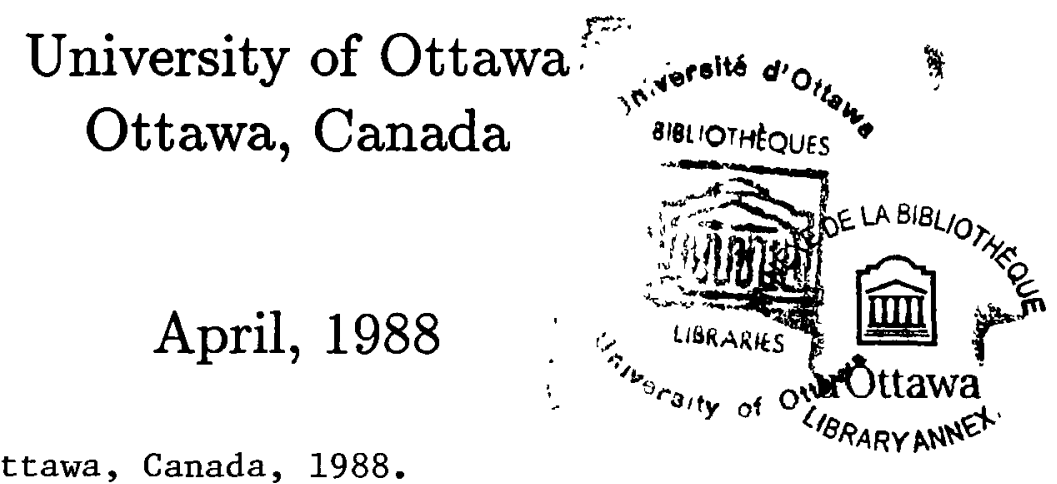

(C) Samsir Tanary, Ottawa, Canada, 1988. 
UMI Number: EC56146

\section{INFORMATION TO USERS}

The quality of this reproduction is dependent upon the quality of the copy submitted. Broken or indistinct print, colored or poor quality illustrations and photographs, print bleed-through, substandard margins, and improper alignment can adversely affect reproduction.

In the unlikely event that the author did not send a complete manuscript and there are missing pages, these will be noted. Also, if unauthorized copyright material had to be removed, a note will indicate the deletion.

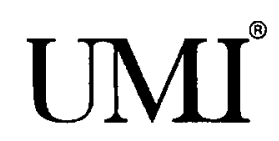

UMI Microform EC56146

Copyright 2011 by ProQuest LLC

All rights reserved. This microform edition is protected against unauthorized copying under Title 17, United States Code.

ProQuest LLC

789 East Eisenhower Parkway

P.O. Box 1346

Ann Arbor, Ml 48106-1346 


\section{ABSTRACT}

This thesis decribes the results of a research project under a work contract with the Structures and Materials Laboratory, NAE, NRC. The objective of the research program is to characterize the mechanical performance of adhesively bonded joints using nondestructive acousto-ultrasonic and destructive shear testing techniques. The research program is intended to assess the feasibility of utilizing acousto-ultrasonics in characterization and strength prediction of adhesively bonded joints.

The basic principles of the acousto-ultrasonic technique are described. The instruments, operating procedures and factors affecting acousto-ultrasonic measurements are explained. Factors affecting the joint strength such as the material and geometry of the joint are also discussed.

Experimental results obtained in a series of characterization tests on adhesively bonded specimens are presented. Single lap joint specimens were made of graphite/epoxy or steel adherends and American Cynamid FM300 adhesive film. Controlled degradation in the bond line strength was achieved by deliberate creation of voids or unbonds, adhesive aging and exposure to elevated temperatures. Good correlations were found between the acoustoultrasonic parameter and the average shear strength of the lap joint specimens. It was also found that the acousto-ultrasonic technique could be employed on foam core aluminium bonded sandwich panels to detect voids, fatigue and impact damaged areas, as well as variation in fabrication process. 


\section{PREFACE}

This thesis entitled "Characterization of Adhesively Bonded Joints" contains results of a research program that was being carried out at NAE Structures and Materials Laboratory. This thesis is intended to provide descriptive information on the feasibility of utilizing nondestructive acousto-ultrasonic technique to quantitatively evaluate adhesively bonded joints. The present research project relates to the use of this nondestructive technique to evaluate the variations in mechanical performance of the bonded joints or foam core aluminium bonded panels due to fabrication variations or exposure to environmental conditions.

In this thesis, the reader is given the information about the applications of adhesively bonded joints, their advantages and limitations. The plan of the research is divided into five phases: (i) Review of literature; (ii) Selection of materials, design and fabrication of test specimen; (iii) Nondestructive evaluation of test specimens; (iv) Mechanical testing and fractographic analysis and (v) Correlation of results. The opening chapter presents an introduction to the statement of problem in order for the reader to comprehend the purpose of the present research.

In Chapter 2, nondestructive techniques available today for adhesive bond quality inspection are reviewed. The basic principles of each technique are briefly described. Comparisons of these nondestructive techniques are then made in terms of their abilities to inspect bond quality and their practicality.

In Chapter 3, a brief review of the applications of acousto- ultrasonic technique to adhesively bonded joints and other structures are presented. The basic principles of the technique are described and factors that may affect the acousto-ultrasonic measurements are discussed. 
In Chapter 4, factors affecting joint strength such as the material and geometry of the bonded joints are analysed for the test specimens used in the present investigation. The analysis is carried out by using theoretical elastic and elastic-plastic solutions obtained by others.

In Chapter 5, experimental results of the characterization of bonded joint specimens in graphite/epoxy composite materials are presented. Controlled degradation in the bond line strength is achieved by deliberately creating voids or unbonds in the joints during fabrication or by aging the uncured adhesive film in room temperature before bonding.

In Chapter 6, experimental results of the characterization of bonded joint specimens in steel are presented. Controlled degradation in the bond line strength is achieved by subjecting the test specimens at elevated temperatures or cyclic thermal exposure. An analysis of wave propagation through the adherend-adhesive system, which supports the experimental data, is also discussed.

Chapter 7 provides experimental results obtained from the use of acousto-ultrasonic technique to inspect foam core aluminium panels in detection of fatigue or impact damaged areas as well as variation in fabrication process. Comparisons with other nondestructive testing techniques in terms of detecting the flaws and damaged areas will be mentioned.

Chapter 8 presents the experimental results obtained by using acousto-ultrasonic technique to monitor the change in stress wave propagation characteristics in adhesively bonded joint specimens under tensile loading. An approach based on the acousto-ultrasonic waveform partitioning method used to study the components of AU waveforms in certain frequency ranges is also presented.

In Chapter 9, general concluding remarks of the results of this thesis are given. Problems regarding the use of acousto- ultrasonic technique that still require further investigations are outlined and recommendations for future work are also presented. 


\section{ACKNOWLEDMENT}

The author is indebted to those people who have given their valuable assistance and suggestions in the development of this thesis. The author wishes to express his sincere gratitude to his research supervisor, Prof. Y. M. Haddad of University of Ottawa. The succesful completion of this thesis depended significantly on his useful ideas and constant encouragement to the author. The author also wishes to express his deep appreciation to Dr. A. Fahr and Mr. S. Lee of Structures and Materials Laboratory, NAE, NRC for their patient guidance and supervision throughout this research. The assistance from the staff members of Structures and Materials laboratory is greatly appreciated. The author also would like to specially thank the staff members of Department of Mechanical Engineering, University of Ottawa for their help and suggestions.

The author is greatly grateful to the National Aeronautical Establishment and Natural Science and Engineering Research Council for the financial assistance through the work contract with University of Ottawa. 


\section{TABLE OF CONTENTS}

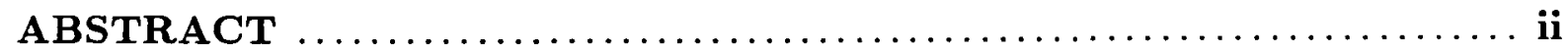

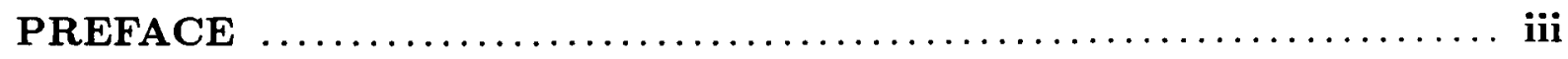

ACKNOWLEDGEMENT $\ldots \ldots \ldots \ldots \ldots \ldots \ldots \ldots \ldots \ldots \ldots \ldots \ldots, \mathbf{v}$

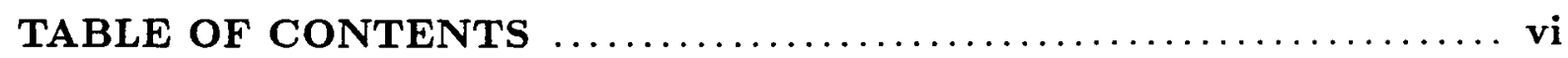

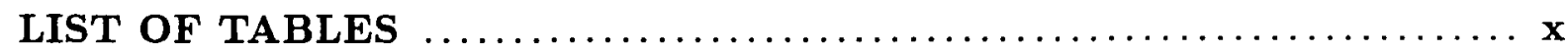

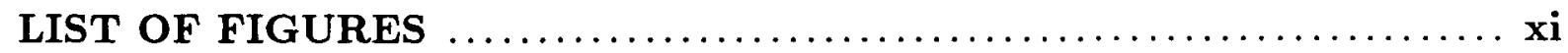

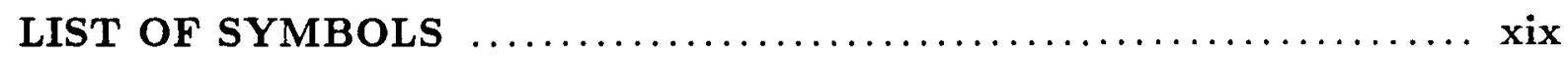

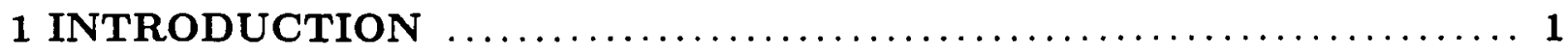

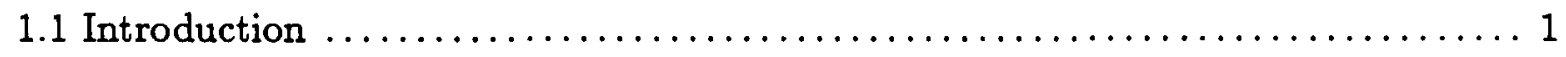

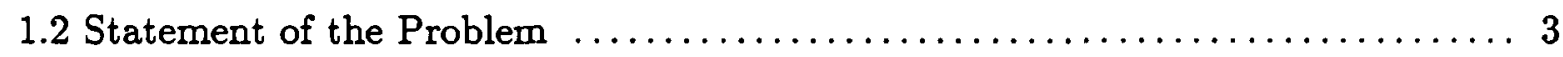

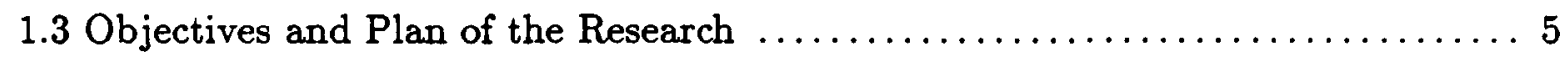

2 REVIEW OF NDT TECHNIQUES FOR ADHESIVELY

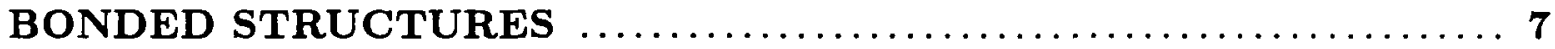

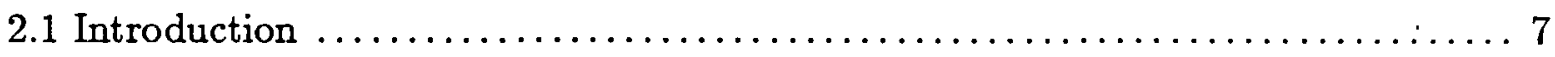

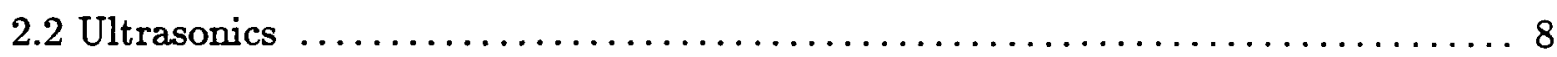

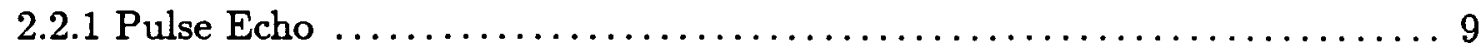

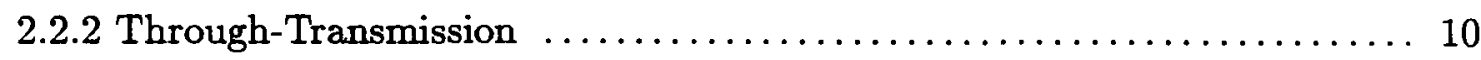

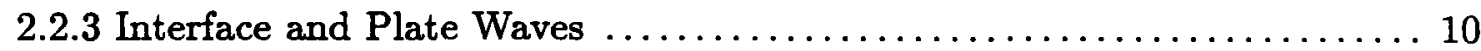

2.2.4 Resonance $\ldots \ldots \ldots \ldots \ldots \ldots \ldots \ldots \ldots \ldots \ldots \ldots \ldots \ldots \ldots \ldots \ldots, 11$

2.2.5 Ultrasonic Spectroscopy $\ldots \ldots \ldots \ldots \ldots \ldots \ldots \ldots \ldots \ldots \ldots \ldots \ldots \ldots, 12$

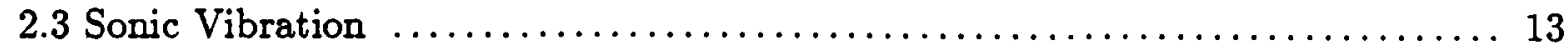

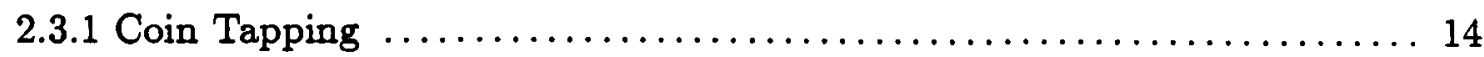

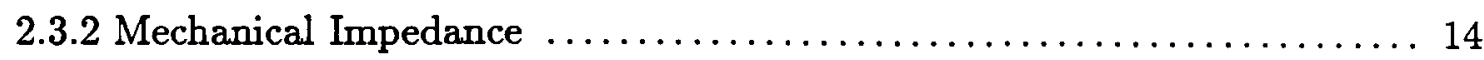

2.3.3 Membrane Resonance $\ldots \ldots \ldots \ldots \ldots \ldots \ldots \ldots \ldots \ldots \ldots \ldots \ldots \ldots \ldots$

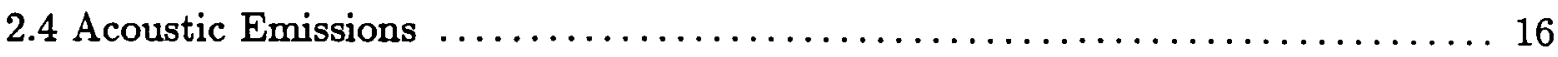

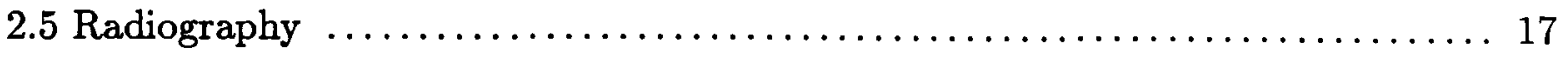




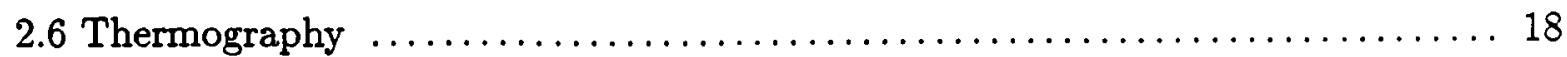

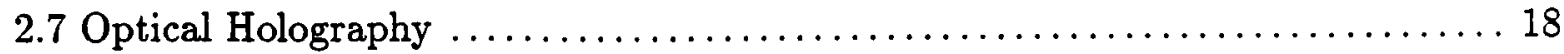

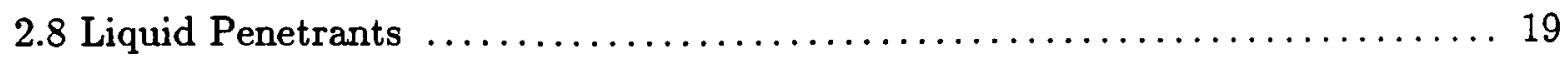

2.9 Comparisons of NDT Methods for

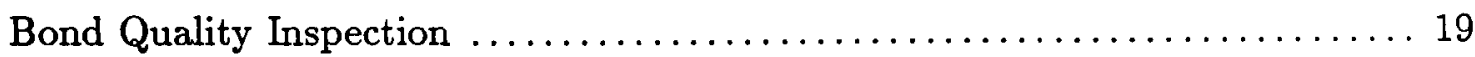

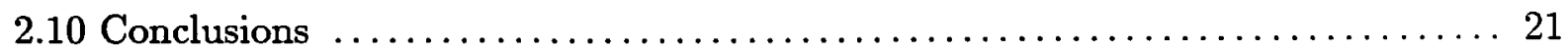

3 ACOUSTO-ULTRASONIC TECHNIQUE $\ldots \ldots \ldots \ldots \ldots \ldots \ldots \ldots \ldots 22$

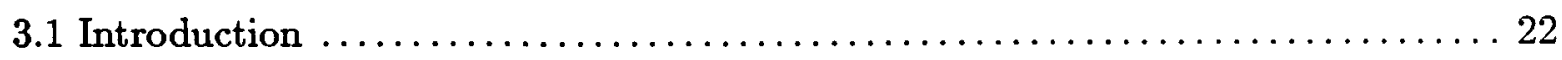

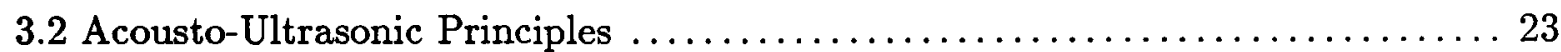

3.3 Factors Affecting Acousto-Ultrasonic Data ........................ 25

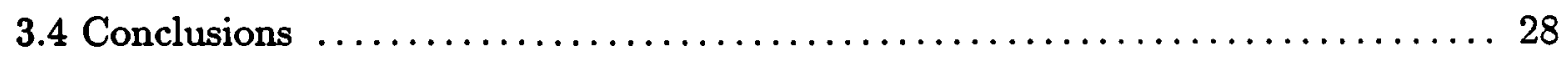

4 FACTORS AFFECTING JOINT STRENGTH $\ldots \ldots \ldots \ldots \ldots \ldots \ldots \ldots 29$

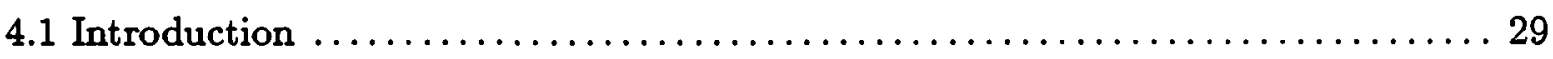

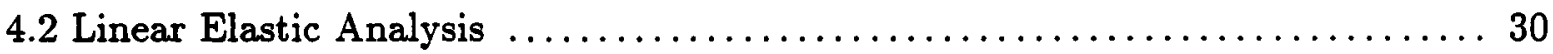

4.2.1 Volkersen's Analysis ................................... 30

4.2.2 Goland and Reissner's Analysis . . . . . . . . . . . . . . . . . . 31

4.2.3 Later Work on Elastic Analysis . .......................... 32

4.2.4 Effect of Parametric Variations

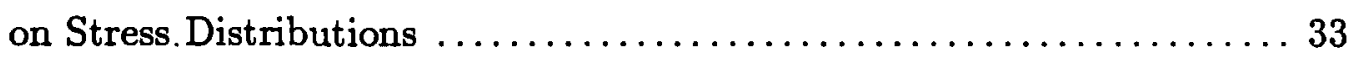

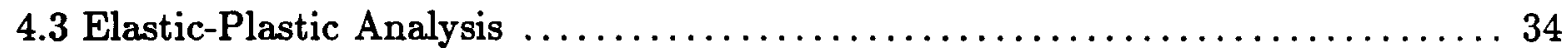

4.3.1 Hart-Smith's Analysis $\ldots \ldots \ldots \ldots \ldots \ldots \ldots \ldots \ldots \ldots \ldots \ldots \ldots \ldots, \ldots \ldots$

4.3.2 Effect of Parametric Variations

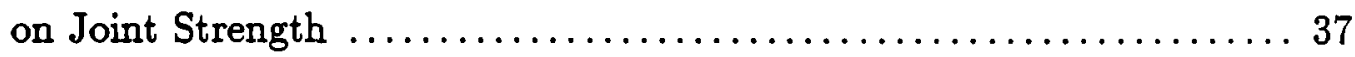

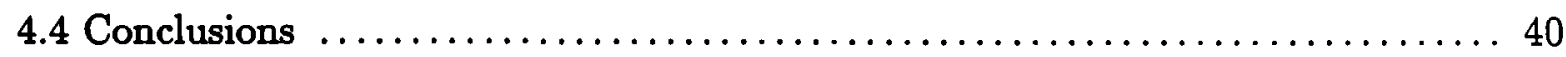

5 CHARACTERIZATION OF ADHESIVELY BONDED JOINTS

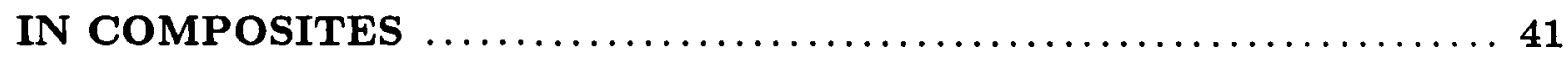

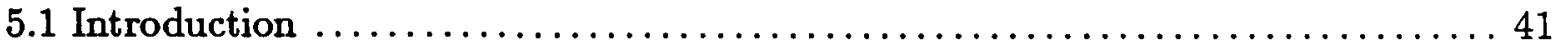

5.2 Experimental Procedure and Apparatus ......................... 42

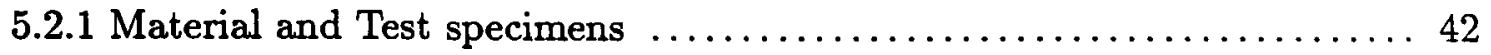


5.2 .2 Acousto-Ultrasonic System $\ldots \ldots \ldots \ldots \ldots \ldots \ldots \ldots \ldots \ldots \ldots \ldots \ldots 4$

5.2 .3 Mechanical Testing and Fractography $\ldots \ldots \ldots \ldots \ldots \ldots \ldots \ldots \ldots . \ldots 45$

5.3 Experimental Results and Discussions $\ldots \ldots \ldots \ldots \ldots \ldots \ldots \ldots \ldots \ldots \ldots$

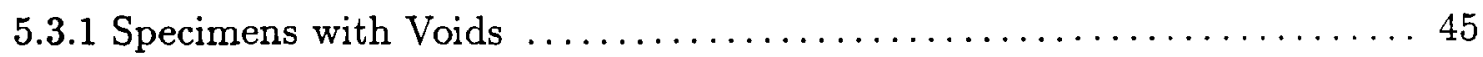

5.3.2 Specimens with Unbonds $\ldots \ldots \ldots \ldots \ldots \ldots \ldots \ldots \ldots \ldots \ldots \ldots . \ldots \ldots$

5.3.3 The Effect of Adhesive Aging ............................ 48

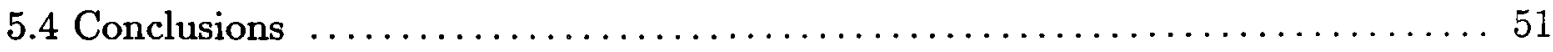

6 EVALUATION OF STEEL BONDED JOINTS AT

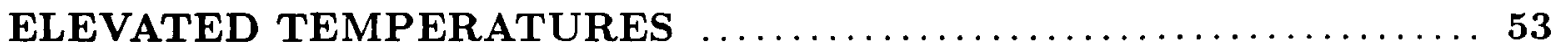

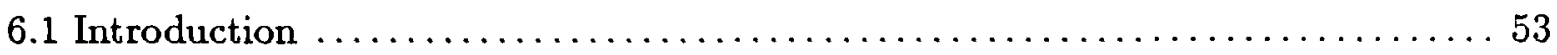

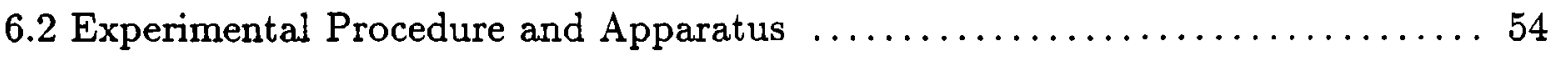

6.2.1 Material and Test Specimens ............................ 54

6.2.2 Acousto-Ultrasonic Measurement Apparatus ................... 55

6.2.3 Mechanical Testing and Fractography $\ldots \ldots \ldots \ldots \ldots \ldots \ldots \ldots \ldots, \ldots \ldots$

6.3 Experimental Results .................................... 57

6.3.1 Effect of Change in Temperature ........................ 57

6.3.2 Effect of Cyclic Thermal Exposure $\ldots \ldots \ldots \ldots \ldots \ldots \ldots \ldots \ldots \ldots . \ldots . \ldots . \ldots$

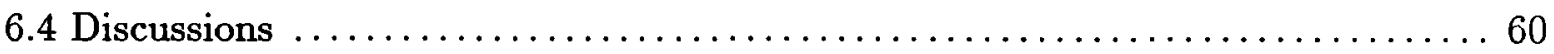

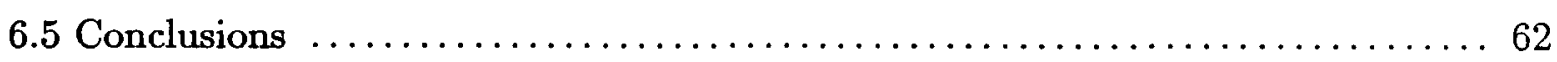

7 EVALUATION OF FOAM CORE ALUMINIUM PANELS $\ldots \ldots \ldots \ldots 63$

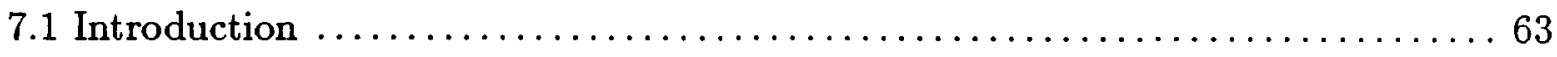

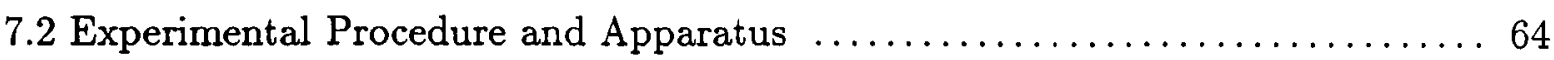

7.2.1 Test Panels ......................................... 64

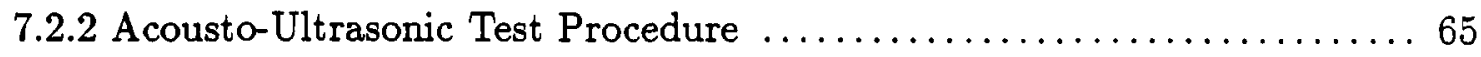

7.2.3 Mechanical Testing and Fractography $\ldots \ldots \ldots \ldots \ldots \ldots \ldots \ldots \ldots \ldots$

7.3 Experimental Results and Discussions $\ldots \ldots \ldots \ldots \ldots \ldots \ldots \ldots \ldots \ldots \ldots \ldots \ldots$

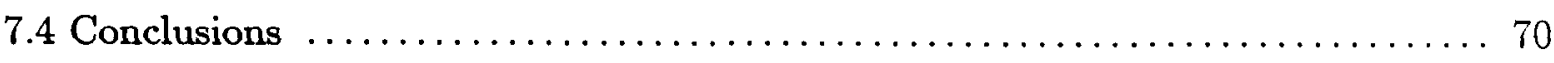

8 EVALUATION OF BONDED JOINTS UNDER

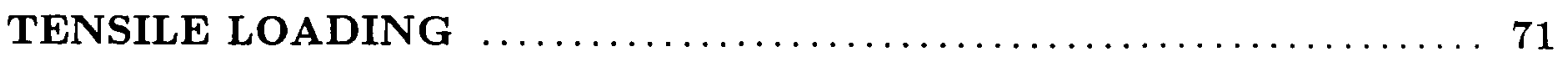




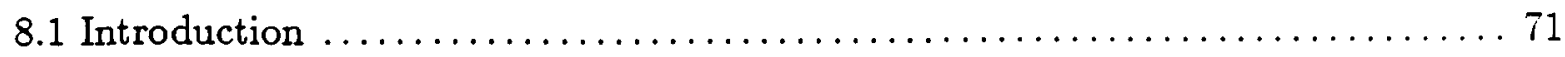

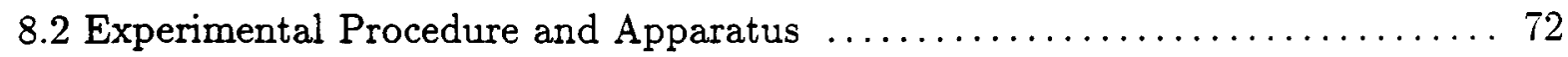

8.2.1 Material and Test Specimens ............................ 72

8.2.2 Acousto-Ultrasonic System Arrangement $\ldots \ldots \ldots \ldots \ldots \ldots \ldots \ldots \ldots . \ldots 3$

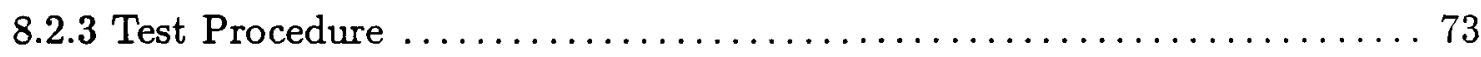

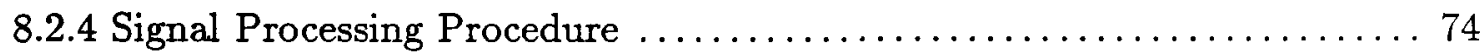

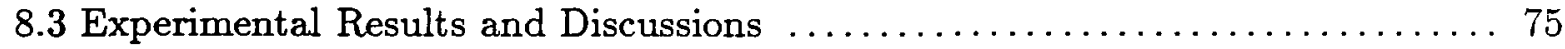

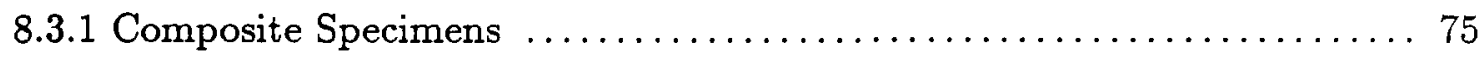

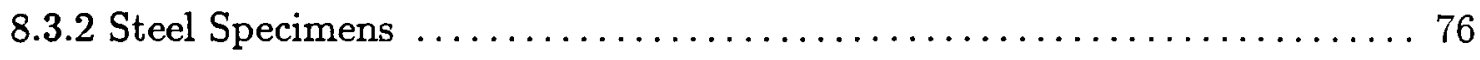

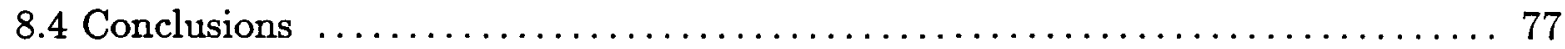

9 CONCLUDING REMARKS AND RECOMMENDATIONS ......... 79

REFERENCES $\ldots \ldots \ldots \ldots \ldots \ldots \ldots \ldots \ldots \ldots \ldots \ldots \ldots \ldots \ldots \ldots \ldots, \mathbf{8 2}$

APPENDIX $A \ldots \ldots \ldots \ldots \ldots \ldots \ldots \ldots \ldots \ldots \ldots \ldots \ldots \ldots \ldots \ldots \ldots, 93$

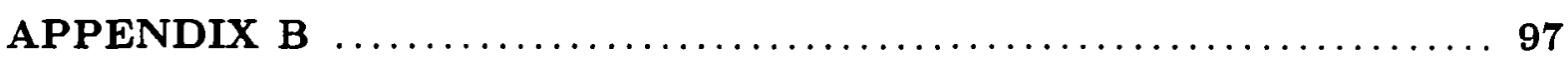




\section{LIST OF TABLES}

Table

3.1 Reproducibility of acousto-ultrasonic data in terms of ring-down count ( $\mathrm{RDC}$ ) and peak amplitude (PA) measurements ......... 105

4.1 Material properties used in theoretical analysis of stress distributions and strength predictions

5.1 Summary of the destructive testing results for specimens with voids

5.2 Summary of the destructive testing results for specimens with unbonds

6.1 Effect of cyclic thermal exposure on acousto-ultrasonic parameter and average shear strength

7.1 Flaw types and description of flaw sites

7.2 The AUP data and the destructive test results of the foam core aluminium panels

8.1 Fracture loads of the composite and steel specimens 


\section{LIST OF FIGURES}

Figure

1.1 Physical discontinuities that may occur in an adhesively bonded joint ..... 114

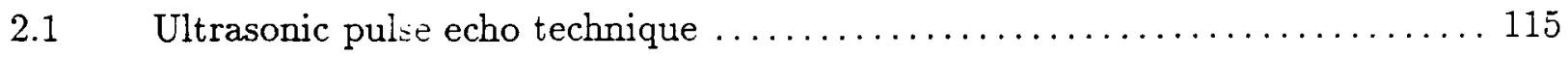

$2.2 \quad$ Ultrasonic through-transmission technique $\ldots \ldots \ldots \ldots \ldots \ldots \ldots \ldots \ldots$

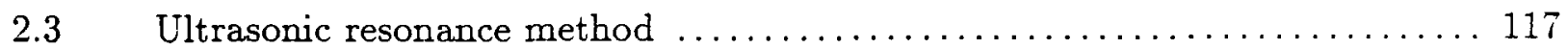

2.4 Simplified diagram of a typical acoustic emission system $\ldots \ldots \ldots \ldots \ldots \ldots 118$

3.1 A schematic diagram of the acousto-ultrasonic test set-up . . . . . . . 119

3.2 Parameters measurable from an acousto-ultrasonic waveform . . . . . . . 120

3.3 An illustration of acousto-ultrasonic parameter (AUP) calculation ........ 121

3.4 The transducer-specimen holding fixture designed for improved

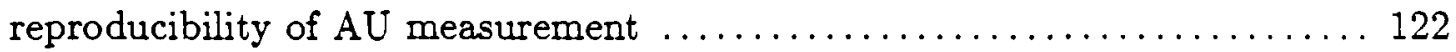

3.5 The change in AUP with respect to time for different coupling fluids used between the transducers and the specimen

3.6 (a) A typical waveform obtained from a bonded joint in graphite/epoxy composites with a resonant condition; (b) Enlargement of the early portion of the waveform in (a)

3.7 (a) A typical waveform obtained from a bonded joint in graphite/epoxy composites with an off-resonant condition; (b) Enlargement of the early

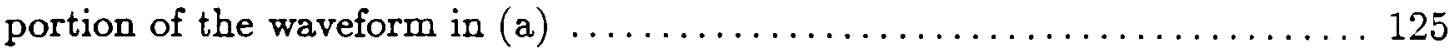

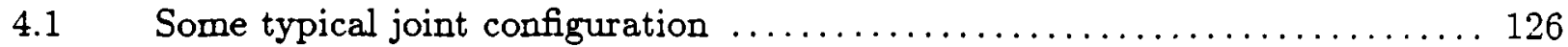

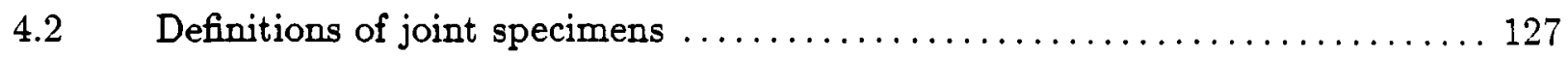

4.3 Adhesive shear stress distribution of a steel lap joint for different loads obtained using Equation A.13 with $\mathrm{L}=76.2 \mathrm{~mm} ; l=12.7 \mathrm{~mm}$;

$\mathrm{h}=0.178 \mathrm{~mm}$ and $\mathrm{t}=2.667 \mathrm{~mm}$

4.4 Adhesive peel stress distribution of a steel lap joint for different loads obtained using Equation A.14 with $\mathrm{L}=76.2 \mathrm{~mm} ; l=12.7 \mathrm{~mm}$; $\mathrm{h}=0.178 \mathrm{~mm}$ and $\mathrm{t}=2.667 \mathrm{~mm}$ 
4.5 Adhesive shear stress distribution of a unidirectional CFRP (composite fibre reinforced plastics) lap joint for different loads obtained using Equation A.13 with $\mathrm{L}=76.2 \mathrm{~mm} ; l=12.7 \mathrm{~mm} ; \mathrm{h}=0.178 \mathrm{~mm}$ and $\mathrm{t}=2.667 \mathrm{~mm}$

4.6 Adhesive peel stress distribution of a unidirectional CFRP lap joint for different loads obtained using Equation A.14 with $\mathrm{L}=76.2 \mathrm{~mm}$; $l=12.7 \mathrm{~mm} ; \mathrm{h}=0.178 \mathrm{~mm}$ and $\mathrm{t}=2.667 \mathrm{~mm}$

4.7 Adhesive shear stress distribution of unidirectional CFRP lap joints for various adhesive thicknesses obtained using Equation A.13 with $\mathrm{L}=76.2 \mathrm{~mm} ; l=12.7 \mathrm{~mm} ; \mathrm{T}=174.8 \mathrm{kN} / \mathrm{m}$ and $\mathrm{t}=2.667 \mathrm{~mm}$

4.8 Adhesive peel stress distribution of unidirectional CFRP lap joints for various adhesive thicknesses obtained using Equation A.14 with $\mathrm{L}=76.2 \mathrm{~mm} ; l=12.7 \mathrm{~mm} ; \mathrm{T}=174.8 \mathrm{kN} / \mathrm{m}$ and $\mathrm{t}=2.667 \mathrm{~mm}$

4.9 Adhesive shear stress distribution of unidirectional CFRP lap joints for several adherend thicknesses obtained using Equation A.13 with $\mathrm{L}=76.2 \mathrm{~mm} ; l=12.7 \mathrm{~mm} ; \mathrm{T}=174.8 \mathrm{kN} / \mathrm{m}$ and $\mathrm{h}=0.178 \mathrm{~mm}$

4.10 Adhesive peel stress distribution of unidirectional CFRP lap joints for several adherend thicknesses obtained using Equation A.14 with $\mathrm{L}=76.2 \mathrm{~mm} ; l=12.7 \mathrm{~mm} ; \mathrm{T}=174.8 \mathrm{kN} / \mathrm{m}$ and $\mathrm{h}=0.178 \mathrm{~mm}$

4.11 Adhesive shear stress distribution of unidirectional CFRP lap joints for various overlap lengths obtained using Equation A.13 with $\mathrm{L}=76.2 \mathrm{~mm} ; \mathrm{t}=2.667 \mathrm{~mm} ; \mathrm{T}=174 \mathrm{kN} / \mathrm{m}$ and $\mathrm{h}=0.178 \mathrm{~mm}$

4.12 Adhesive peel stress distribution of unidirectional CFRP lap joints for various overlap lengths obtained using Equation A.13 with $\mathrm{L}=76.2 \mathrm{~mm} ; \mathrm{t}=2.667 \mathrm{~mm} ; \mathrm{T}=174.8 \mathrm{kN} / \mathrm{m}$ and $\mathrm{h}=0.178 \mathrm{~mm}$

4.13 Elastic-plastic model representation of the actual American Cynamid

FM300K adhesive shear stress-strain property [16]

4.14 Influence of adhesive thickness upon the theoretical shear strength of unidirectional CFRP lap joints for several adherend thicknesses 
with $\mathrm{L}=76.2 \mathrm{~mm}$ and $l=12.70 \mathrm{~mm}$

4.15 Schematic presentation of the effect of elastic-plastic, as compared

with purely elastic behaviour of the adhesive on the stress distribution

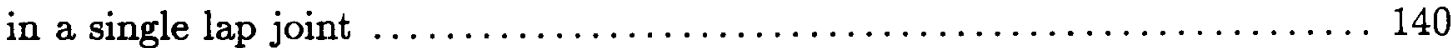

4.16 Effect of adhesive thickness on the theoretical shear strength

of unidirectional CFRP lap joints for several overlap lengths with

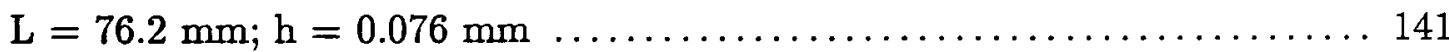

4.17 Effect of adhesive thickness on the theoretical shear strength

of unidirectional CFRP lap joints for several overlap lengths with

$\mathrm{L}=76.2 \mathrm{~mm} ; \mathrm{h}=0.229 \mathrm{~mm}$

4.18 Theoretical peel strength of unidirectional CFRP lap joints as

a function of adhesive thickness for various adherend thickness with

$\mathrm{L}=76.2 \mathrm{~mm}$ and $l=12.7 \mathrm{~mm}$

4.19 Theoretical peel strength of unidirectional CFRP lap joints as

a function of adherend thickness for various overlap lengths with

$\mathrm{L}=76.2 \mathrm{~mm}$ and $\mathrm{h}=0.076 \mathrm{~mm}$

4.20 Theoretical peel strength of unidirectional CFRP lap joints as

a function of adherend thickness for various overlap lengths with

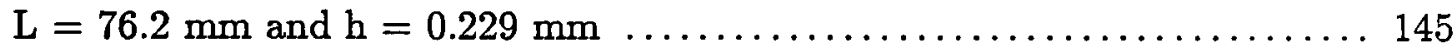

4.21 Influence of adherend thickness upon the adherend bending strength

of unidirectional CFRP lap joints with $\mathrm{L}=76.2 \mathrm{~mm}$ and $\mathrm{h}=0.076 \mathrm{~mm} \quad . .146$

5.1 Ultrasonic c-scan result of panel no.1 (specimen type A) . ............. 147

$5.2 \quad$ Ultrasonic c-scan result of panel no.2 (specimen type B) $\ldots \ldots \ldots \ldots \ldots 148$

$5.3 \quad$ Test specimen configurations (dimensions are in $\mathrm{mm}$ ) $\ldots \ldots \ldots \ldots \ldots \ldots . \ldots 149$

$5.4 \quad$ Ultrasonic c-scan result of specimens with unbonds $\ldots \ldots \ldots \ldots \ldots \ldots \ldots$

5.5 A schematic diagram of the acousto-ultrasonic system as used in the

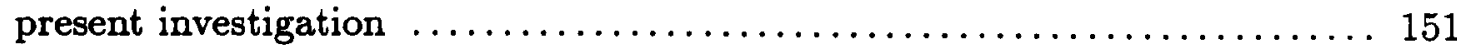

5.6 The frequency spectrum of the transmitting transducer (AET-FC500) … 152

5.7 The frequency spectrum of the receiving transducer (AET-AC375) . . . . . 153 
5.8 Input pulse to the transmitting transducer for $\mathrm{AU}$ measurement $\ldots \ldots \ldots 154$

5.9 (a) A typical acousto-ultrasonic waveform for a relatively good specimen ... 155

5.9 (b) Frequency spectrum of the acousto-ultrasonic waveform shown in

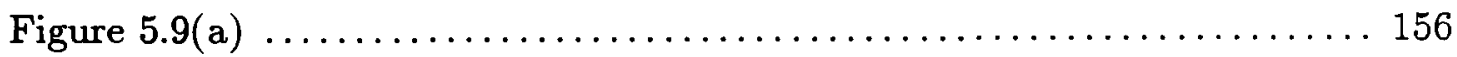

5.10 (a) A typical acousto-ultrasonic waveform for a specimen with

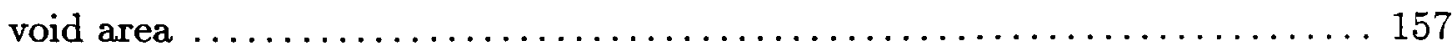

5.10 (b) Frequency spectrum of the acousto-ultrasonic waveform shown in

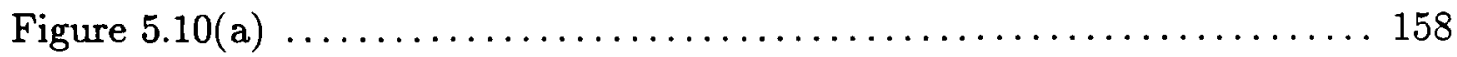

5.11 The normalized acousto-ultrasonic parameter (AUP) vs the average shear strength for specimens with voids $\ldots \ldots \ldots \ldots \ldots \ldots \ldots \ldots \ldots \ldots$

5.12 The change in frequency spectra of the acousto-ultrasonic signals from bonded composite joint specimens with increasing void size $\ldots \ldots \ldots \ldots 160$

5.13 The average shear strength vs the void area for single lap composite

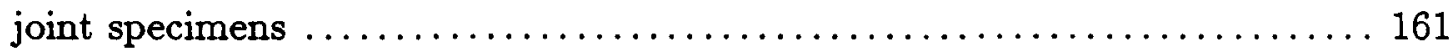

5.14 A typical fracture surface of a specimen without void area;

(b) cohesive failure in the adhesive; (c) interfacial failure; (d) fibre

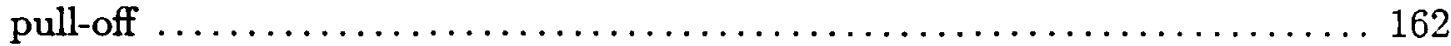

5.15 A typical fracture surface of a specimen with void area across the joint; (b) cohesive failure in the adhesive; (c) interfacial failure; (d) fibre pull-off 163

5.16 A typical fracture surface of a specimen with void area at the edge of the joint; (b) cohesive failure in the adhesive; (c) interfacial failure; (d) fibre pull-off $\ldots \ldots \ldots \ldots \ldots \ldots \ldots \ldots \ldots \ldots \ldots \ldots \ldots \ldots \ldots \ldots$

5.17 An acousto-ultrasonic waveform for a typical specimen without unbonded area

5.18 An acousto-ultrasonic waveform for a typical specimen with unbonded

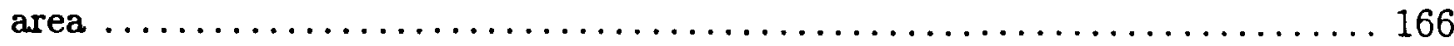

5.19 The correlation between the normalized acousto-ultrasonic parameter (AUP) and the average shear strength of the specimens with unbonds .... 167 
5.20 The change in normalized AUP with respect to the variation in the

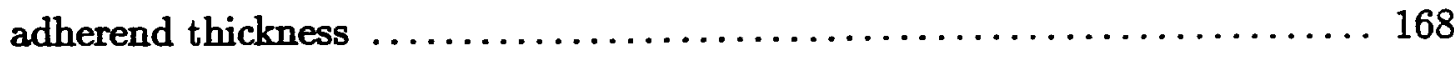

5.21 The change in the frequency spectra of the acousto- ultrasonic signals from bonded joint specimens with increasing unbonded area . ..... 169

5.22 The average shear strength vs the unbonded area for single lap composite joint specimens $\ldots \ldots \ldots \ldots \ldots \ldots \ldots \ldots \ldots \ldots \ldots \ldots \ldots \ldots \ldots \ldots \ldots \ldots \ldots \ldots \ldots, 170$

5.23 Photographs of typical fracture surfaces for specimens with unbonds showing the different failure modes $\ldots \ldots \ldots \ldots \ldots \ldots \ldots \ldots \ldots \ldots \ldots \ldots$

5.24 A typical ultrasonic pulse-echo signal for an adhesively bonded

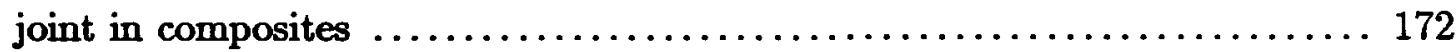

5.25 Adhesive thicknesses of specimens measured with ultrasonic pulse-echo method vs those measured with an optical microscope ........ 173

5.26 The effect of adhesive thickness on the AUP for specimens with (a) 3 weeks of aged adhesive; (b) 5 weeks of aged adhesive $\ldots \ldots \ldots \ldots 174$

5.27 The change in normalized AUP (Equation 5.1) as a function of

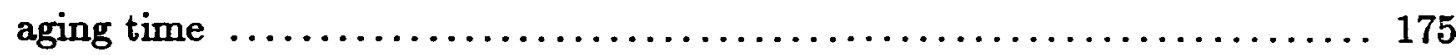

5.28 The change in normalized joint strength (Equation 5.2) as a

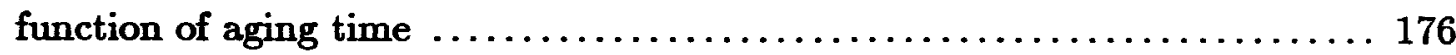

5.29 Plot of the normalized AUP vs the normalized joint strength $\ldots \ldots \ldots \ldots \ldots 177$

5.30 Photographs of fracture surfaces for specimens with aged adhesive

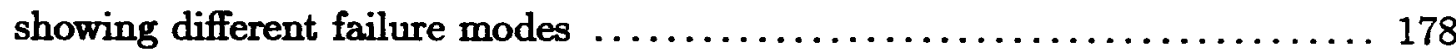

6.1 Acousto-ultrasonic system (a) for room temperature AU measurements and (b) for elevated temperature AU measurements ....... 179

6.2 The change in normalized acousto-ultrasonic parameter (AUP) with increasing temperature $\ldots \ldots \ldots \ldots \ldots \ldots \ldots \ldots \ldots \ldots \ldots \ldots \ldots \ldots \ldots \ldots \ldots \ldots \ldots, 180$

6.3 The change in frequency spectra of the acousto-ultrasonic signal from an adhesively bonded steel specimen with increasing temperature .... 181

6.4 The frequency spectra of the acousto-ultrasonic signal from a steel specimen without adhesive bond at different temperatures $\ldots \ldots \ldots \ldots \ldots 182$ 
6.5 The change in average shear strength of the adhesively bonded steel

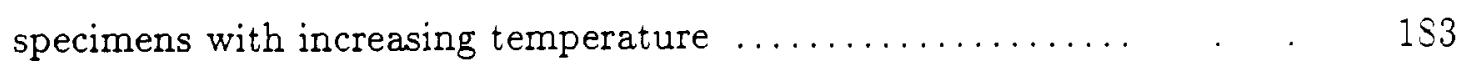

6.6 Fracture surfaces of the specimens tested at (a) $30^{\circ} \mathrm{C}$, (b) $90^{\circ} \mathrm{C}$, (c) $120^{\circ} \mathrm{C}$ and (d) $150^{\circ} \mathrm{C}$. The fracture surfaces generally consist of interfacial and cohesive failures as shown at higher magnification in (e) for $150^{\circ} \mathrm{C}$ and in (f) for $30^{\circ} \mathrm{C}$, respectively $\ldots \ldots \ldots \ldots \ldots \ldots \ldots \ldots$

6.7 Correlation of the acousto-ultrasonic parameter with the average shear strength at different temperatures $\ldots \ldots \ldots \ldots \ldots \ldots \ldots \ldots \ldots \ldots \ldots$

6.8 Correlation of the acousto-ultrasonic parameter with average shear strength of two groups of specimens; as-fabricated specimens and specimens subjected to cyclic thermal exposure $\ldots \ldots \ldots \ldots \ldots \ldots \ldots \ldots \ldots \ldots \ldots$

6.9 Typical fracture surfaces of (a) an as-fabricated specimen as compared with (b) a specimen subjected to cyclic thermal exposure $\ldots \ldots \ldots \ldots \ldots 187$

$7.1 \quad$ A foam core aluminium test specimen $\ldots \ldots \ldots \ldots \ldots \ldots \ldots \ldots \ldots \ldots \ldots \ldots$

7.2 Photographs of test panels after impact at three energy levels using two different impact heads

7.3 A schematic diagram of the acousto-ultrasonic test set-up for evaluating

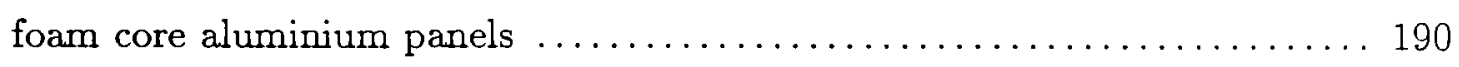

7.4 The acousto-ultrasonic test results for the panel Fo/Al-2 containing

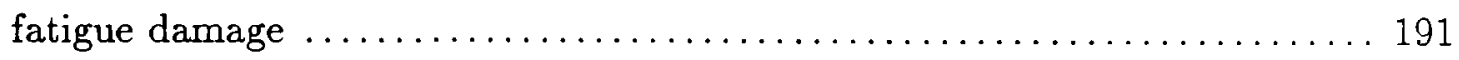

7.5 The acousto-ultrasonic test results for the panel Fo/Al-3 containing three visible impact damage sites introduced at 1.5, 2.9 and 14 joules using a $12.5 \mathrm{~mm}$ diameter semi-spherical impact head

7.6 The acousto-ultrasonic test results for the panel Fo/Al-4 containing two invisible and one visible impact damage sites introduced at $1.4,2.8$ and 13.9 joules using a nearly flat indentor $\ldots \ldots \ldots \ldots \ldots \ldots \ldots \ldots \ldots$

7.7 A typical acousto-ultrasonic waveform for the as-received (AR) panel . . ... 194

7.8 A typical acousto-ultrasonic waveform for the as-received panel

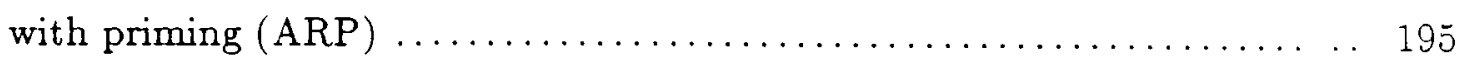


7.9 A typical acousto-ultrasonic waveform for the panel with surface preparation technique of NRP (new roller with priming) $\ldots \ldots \ldots \ldots \ldots \ldots 196$

7.10 Correlation of acousto-ultrasonic parameter (AUP) with peel torque of foam core aluminium panels $\ldots \ldots \ldots \ldots \ldots \ldots \ldots \ldots \ldots \ldots \ldots . \ldots \ldots$

7.11 A representative fracture surface for the as-received (AR) panel ......... 198

7.12 A representative fracture surface for the panel with old roller (OR) surface preparation technique ..................................... 199

7.13 A representative fracture surface for the panel with new roller

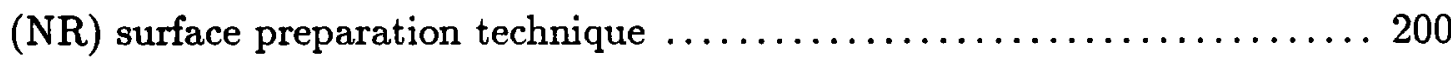

7.14 A representative fracture surface for the panel with new roller surface preparation technique and with two layers of adhesive (NR2) .......... 201

7.15 A representative fracture surface for the as-received panel

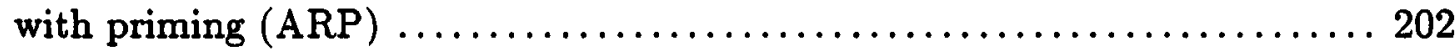

7.16 A representative fracture surface for the panel with surface preparation technique of ORP (old roller with priming) .............. 203

7.17 A representative fracture surface for the panel with surface preparation technique of NR2P (new roller with 2 layers of adhesive

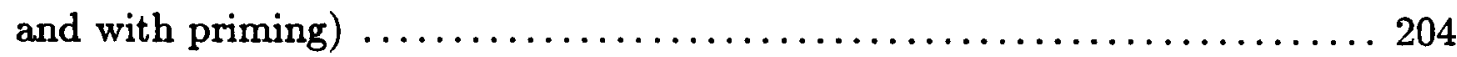

7.18 A representative fracture surface for the panel with surface preparation technique of NRP (new roller with priming) ........ 205

8.1 A schematic diagram of the acousto-ultrasonic monitoring a joint

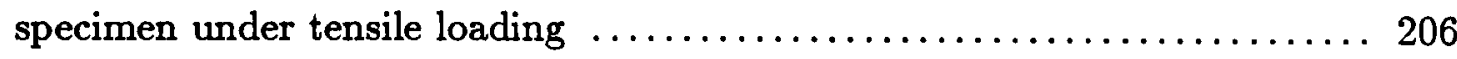

8.2 Apparatus for measuring acousto-ultrasonic parameter (AUP) during

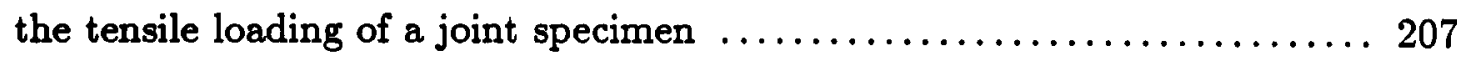

8.3 Constant force clamps are used to hold the transducers to the specimen

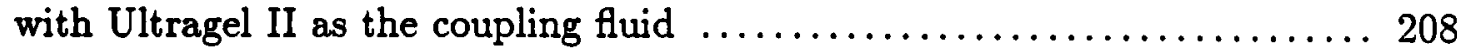

8.4 The frequency spectra of the typical acoustic emission signals when the tensile load applied to a composite joint specimen was about (a) $60 \%$ (b) $75 \%$ (c) $80 \%$ and (d) $95 \%$ of the fracture load .............. 209 
8.5 Typical AU waveforms with frequency content in the range of (a) 0.0 to $1.7 \mathrm{MHz}$ (b) 0.0 to $0.275 \mathrm{MHz}$ (c) 0.275 to $0.550 \mathrm{MHz}$ and (d) 0.550 to $0.875 \mathrm{MHz}$ for a representative bonded joint in composites .......... 210

8.6 The normalized AUP of the waveforms with four different ranges of frequency content vs the normalized load for a representative composite

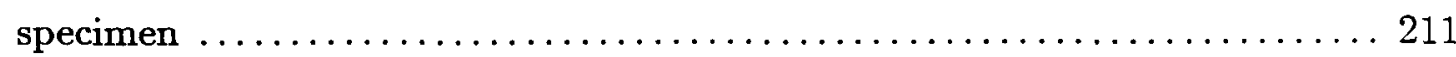

8.7 The normalized AUP of the waveforms with frequency content in the range 0.550 to $0.875 \mathrm{MHz}$ vs the normalized load for composite specimens . 212

8.8 A typical acousto-ultrasonic waveform in (a) time domain and (b) frequency domain signals for a representative bonded joint specimen in steel ....... 213

8.9 Typical acousto-ultrasonic waveforms with frequency content in the range of (a) 0.0 to $1.7 \mathrm{MHz}$ (b) 0.0 to $0.4 \mathrm{MHz}$ (c) 0.4 to $0.8 \mathrm{MHz}$ and (d) 0.8 to $1.2 \mathrm{MHz}$ for a representative steel bonded joint specimen ...... 214

8.10 The normalized AUP of the waveforms with four different ranges of frequency content vs the normalized load for a representative steel specimen ....

8.11 The normalized AUP of the waveforms with frequency content in the range of 0.4 to $0.8 \mathrm{MHz}$ vs the normalized load for steel specimens 


\title{
LIST OF SYMBOLS
}

\author{
AUP acousto-ultrasonic parameter \\ $b \quad$ joint width \\ $d$ length of the elastic zone in adhesive bond \\ $D_{t} \quad$ acoustic energy transmission coefficent \\ $E \quad$ Young's modulus of the adherend \\ $E_{a} \quad$ Young's modulus of the adhesive layer \\ $f$ frequency \\ $F \quad$ harmonic force input \\ $G_{a} \quad$ shear modulus of the adhesive layer \\ $h$ thickness of the adhesive layer \\ $k \quad$ constant defined in Equation A.9 \\ $k^{\prime} \quad$ constant defined in Equation A.10 \\ $k_{1} \quad$ constant defined in Equation A.21 \\ $k_{1}^{\prime} \quad$ constant defined in Equation A.22 \\ $k_{2}$ bending moment factor defined in Equation 4.8 \\ $k_{b} \quad$ bending stiffness parameter \\ $K \quad$ constant defined in Equation 4.13 \\ $l$ half overlap length \\ $M_{o} \quad$ internal bending moment per unit joint width \\ $R_{1} \quad$ constant defined in Equation A.6 \\ $R_{2} \quad$ constant defined in Equation A.7 \\ $R_{3} \quad$ constant defined in Equation A.8
}




\begin{tabular}{ll} 
SWF & stress wave factor defined in Equation 3.1 \\
$t$ & thickness of the adherend \\
$T$ & load per unit joint width \\
$T_{0}$ & internal axial force resultant per joint width \\
$U_{1}$ & constant defined in Equation A.11 \\
$U_{2}$ & constant defined in Equation A.12 \\
$U_{1}^{\prime}$ & constant defined in Equation A.27 \\
$U_{2}^{\prime}$ & constant defined in Equation A.28 \\
$v$ & velocity \\
$V_{o}$ & internal shear force resultant per joint width \\
$Z$ & mechanical impedance \\
$Z_{1}$ & acoustic impedance in material 1 \\
$\gamma$ & constant defined in Equation A.5 \\
$\gamma_{e}$ & elastic adhesive shear strain \\
$\gamma_{p}$ & plastic adhesive shear strain \\
$\delta$ & constant defined in Equation 4.2 \\
$\delta^{\prime}$ & constant defined in Equation A.3 \\
$\delta^{\prime \prime}$ & constant defined in Equation A.17 \\
$\lambda$ & constant defined in Equation A.4 \\
$\lambda_{1}$ & constant defined in Equation A.19 \\
$\lambda_{2}$ & constant defined in Equation 4.5 \\
$\lambda_{D}$ & the acoustic wavelength \\
$\mu$ & poisson's ratio \\
$\phi$ & constant defined in Equation A.26 \\
$\rho$ & density \\
$\sigma_{a v}$ & average normal stress \\
$\sigma_{m a x}$ & maximum adherend bending stress \\
$\sigma_{y}$ & peel stress \\
$\sigma_{y(m a x)}$ & maximum peel stress \\
\hline
\end{tabular}


$\begin{array}{ll}\tau & \text { shear stress } \\ \tau_{a p p} & \text { shear stress (applied) } \\ \tau_{a v} & \text { average shear stress } \\ \tau_{\text {max }} & \text { maximum shear strength } \\ \tau_{p} & \text { plastic adhesive shear stress } \\ \chi & \text { constant defined in Equation } 4.17 \\ \xi & \text { constant defined in Equation } 4.15 \\ \zeta & \text { constant defined in Equation A.18 }\end{array}$ 


\section{Chapter 1}

\section{INTRODUCTION}

\section{$1.1 \quad$ Introduction}

Ideally, a structure should be designed without joints, but a structure without joints generally would not be feasible due to its high cost of manufacturing and excess weight. The designer has essentially two basic techniques for joining components in a structure, namely, mechanical fastening and adhesive bonding.

In the past, only mechanical joining techniques were used for structural components and adhesive bonding methods were mostly utilized in nonstructural applications. In the 1940s, adhesives became more important in structural bonding when the aircraft industry promoted their use as structural bonding agents. In those times, casein adhesives were utilized for bonding joints in wooden aircraft constructions, but the adhesives were later found to have poor resistance to moisture and mould growth [1]. The subject of adhesives became even more interesting to scientists when the application of synthetic resins as adhesives for wood, rubber, glass and metals were discovered [2]. A major development in adhesively bonded joints has been the use of mixtures of synthetic resins to make aluminium alloy structures for aircraft. The advantage of the synthetic resins over the natural-type adhesives is in their excellent resistance to moisture, mould growth and other hazardous service conditions. In 1950s, new epoxy resin-based adhesives were first introduced [3]. These adhesives provide high cohesive strength and, in addition, their excellent creep resistance 
properties make them ideal for structural applications.

Today, the application of adhesives has expanded widely. The use of adhesives in paper, packaging, woodworking and footwear industries still remains important. However, it has extended its significance in industrial equipment, building and construction, vehicle manufacture, electrical and optical assemblies, military and aerospace applications.

A rapid increase in the use of adhesively bonded joints in structural or nonstructural components as a replacement for conventional mechanical joints in recent years could be accounted for the fact that designers and engineers are searching for lighter and more economical structural designs. The application of bonded joints in structural components results in cost savings in assembly and manufacturing due to reduced material requirements, weight reduction, joining of several components at the same time, and simplified assembly procedures. Besides, the use of adhesively bonded joints permits adaptation to many production processes due to the versatility of adhesive forms and methods of application. It also results in more uniform distribution of stresses over the entire bonded area due to minimization and distribution of the stress concentrations which commonly occur with mechanical fasteners. In addition, gaps and protruding mechanical fasteners such as bolts and rivets are eliminated to give an improved appearance of finished product with smooth external surfaces and contours. With bonded joints, it is possible to join various types of materials which may be different in composition, coefficients of expansion, moduli and thickness. It is also possible to join complex shapes, thin sheets of materials as well as heat-sensitive materials, where other joining methods are not possible. Furthermore, an adhesive layer in a bonded joint can be used to act as either an insulator or conductor depending on the choice of the designer by specifically formulating the adhesive. Adhesives can also be employed to avoid or reduce galvanic corrosion between different materials. Problems of corrosion often arise due to the electromotive-series relationship which occurs when dissimilar materials are joined by other conventional methods. The use of adhesives separates the two joined materials, thus eliminates the problems.

However, as with any technology, there are some disadvantages in using adhesively bonded joints. One of the disadvantages is that it may require complicated bonding pro- 
cesses such as surface preparation of adherends before joining and maintenance of clean components as well as preparation and application of adhesives. During curing of the adhesives, it may require to control processing temperature, pressure and humidity conditions. In some cases, high curing temperatures and different coefficients of thermal expansion of the adhesive and the adherends may result in residual stresses in adhesively bonded joints. Another limitations in the use of bonded joints is that once the joints are assembled, it is usually difficult for bond quality inspection. Also, it is generally difficult or even impossible to dismantle an adhesively bonded structure for repair without destroying the structure itself. In addition, during the service of a bonded joint, its performance may be affected by environmental conditions such as humidity and temperature. Thus, long term durability of adhesive bonding under severe service conditions is difficult to predict. The use of adhesively bonded joints replacing mechanical fasteners may reduce stress concentrations, but bonded joints require careful design to minimize peel, cleavage and thermal stresses.

Although the adhesive bonding technique offers many advantages in joining structural components, its limitations may make it an unattractive alternative. Thus, before deciding to use adhesively bonded joints, it is important to identify problems or limitations that are most critical. In the next section, problems regarding the use of bonded joints are discussed.

\subsection{Statement of the Problem}

The problem of large local stresses in mechanical joints can often be significantly reduced by the use of adhesive bonding, but even in a simple adhesively bonded joint the stress distribution is not uniform. If the adhesive used is a brittle one, the peak local stresses could be many times the average stress and failure could generally occur at a stress much lower than the failure stress determined by the bulk properties of the adhesive. The peak local stresses can approximately be determined by analytical or numerical methods if the dimensions of the joint and the bulk properties of the adhesive and the adherends are known. Both of these methods assume perfect bonding in the joint. However, perfect 
bonding in the joint cannot always be achieved because the bond quality depends on many factors. These factors include bonding technique, cleanliness of surfaces, effectiveness of surface preparation, application and distribution of the adhesive, and the curing process. Consequently, during the manufacture of the joint, defects can easily be introduced in the joint.

There are basically three main types of bond defects : i) physical discontinuities such as voids, unbond or porosity (Figure 1.1) ii) lack of cohesive bond strength iii) poor adhesive bond strength.

Voids in the adhesive are caused either by lack of adhesive or by the presence of foreign matter. An unbond is when there is a gap between the adhesive and the adherend. In the literature, this situation is sometimes referred to as debond, disbond or lack of bond. Porosity is due to air bubbles in the adhesive layer. The cohesive bond strength is the strength of the adhesive layer itself, while the adhesive bond strength is defined as the amount of molecular attraction of the adhesive to the adherend at the adhesive/adherend interface.

One of the major limitations on the use of adhesives in structural applications is associated with the difficulty encountered in making an accurate determination of bond quality or potential performance after the joint has been assembled. Furthermore, the performance of the bonded joint during service may be affected by the environmental conditions. Thus, it is very important that nondestructive evaluation (NDE) methods be available to ensure the structural performance of the bonded joint meets design and service requirements.

Over the past few years, the development of nondestructive evaluation techniques has increased rapidly. Most of the NDE techniques are sensitive to flaws or voids in materials, but few techniques can provide information on the overall performance of the material. Of the few techniques sensitive to material properties, acoustic emission and ultrasonics are the most widely used methods for evaluating material performance. Acousto- ultrasonics (AU) is a relatively new NDE method developed by Vary [4] which combines the beneficial aspects of conventional ultrasonic and acoustic emission techniques. Recent studies by various researchers [4-13] reveal that there is a strong correlation between the stress 
wave propagation characteristics determined by acousto-ultrasonics and various mechanical properties of materials. Based on these previous results, it was considered probable that the acousto-ultrasonic technique might provide information on the overall performance of adhesively bonded joints.

\subsection{Objectives and Plan of the Research}

The primary objective of this research program is to characterize the mechanical performance of adhesively bonded joints by nondestructive acousto-ultrasonic technique and destructive shear testing. In particular, characterization of adhesively bonded joints in terms of their ultimate tensile shear strength affected by various fabrication and service conditions will be carried out. Due to these conditions, different types of bond defects such as physical discontinuities, poor cohesive strength and poor adhesive strength will be introduced in the joints. The effect of each type of these defects on the mechanical performance of the joints will be examined and evaluated.

The research program is intended to assess the feasibility of utilizing acousto-ultrasonic techniques in characterization and strength prediction of adhesively bonded joints. On the initial stage, a nondestructive parameter measurable from an acousto-ultrasonic waveform will be established. Selection of this acousto-ultrasonic parameter is based on its sensitivity to material properties. Factors that affect the measurement of this acousto-ultrasonic parameter will then be identified and studied. Acousto-ultrasonic evaluation of the bonded joints will be analysed in time and frequency domains. The joint strength prediction can be achieved by correlating the acousto-ultrasonic parameter and the joint ultimate tensile shear strength after the measurement system has been calibrated for material and geometrical variations of the joints.

Characterization of the bonded joints by destructive testing will be carried out in order to verify the results of nondestructive evaluation and determine failure modes with analysis of the fracture surfaces of the joints. The relationship between the failure modes and the fracture load will be determined. 
It is the main objective of the present thesis to deal with the nondestructive evaluation of adhesively bonded joints. Accordingly, Chapter 2 of this thesis presents a literature review of nondestructive techniques available today for adhesive bond quality inspection or evaluation. The basic principles of each technique are briefly described. Advantages and limitations in the use of these nondestructive techniques are discussed. In the present research, nondestructive acousto-ultrasonic technique is employed to evaluate adhesively bonded joints. It is therefore considered to be essential for the reader to comprehend the basic principles of this technique and to identify factors that may affect the acousto-ultrasonic measurement. Hence, Chapter 3 is devoted entirely to describe the acousto-ultrasonic technique. However, before attempting to evaluate the bonded joints nondestructively, it is important to identify the factors which may affect the shear strength of the bonded joints. These factors can be examined by using elastic and elastic-plastic theoretical analyses as discussed in Chapter 4.

Fibre reinforced composite materials are normally joined with adhesive bonding, due to the inefficency of mechanical joining methods which usually results in the cutting of fibres. Thus, Chapter 5 deals with the application of acousto-ultrasonic technique to characterize adhesively bonded joints in graphite/epoxy composite materials. In order to obtain variation in joint strength, controlled degradation in the bond strength is accomplished by deliberate introduction of voids or unbonds in the joints, or by adhesive aging. Another application of acousto-ultrasonic technique in the present study is to evaluate adhesively bonded joints in steel at elevated temperatures which is discussed in Chapter 6. Then, Chapter 7 presents the use of acousto-ultrasonic technique to inspect foam core aluminium bonded panels, which may have potential applications in transport vehicles, in detection of fatigue and impact damaged areas as well as variation in fabrication process.

In Chapter 8, the effect of tensile load applied to an adhesively bonded joints on the acousto-ultrasonic stress wave propagation characteristics is studied. Finally, concluding remarks on the presented analysis and future research work are discussed in Chapter 9. 


\section{Chapter 2}

\section{REVIEW OF NDT TECHNIQUES FOR ADHESIVELY BONDED STRUCTURES}

\subsection{Introduction}

An ideal non-destructive testing (NDT) technique would have to be able to provide a direct prediction of the bond strength. But the strength prediction by NDT methods is usually difficult and indirect. It often requires to correlate bond strength with other properties such as stiffness, thickness of bond, and attenuation of signals in the adhesive. In addition, the stress distribution in a typical adhesively bonded joint is not uniform. Thus, the strength is more sensitive to the integrity of some areas of the joint than to others. In many cases, measurement of such properties as stiffness, thickness of bond line, void area, etc does not necessarily provide good correlations with joint strength. However, variations in these properties do give an indication that a joint may be defective.

As explained in Chapter 1, three types of defects can occur in an adhesively bonded joint, namely, physical discontinuities, poor cohesive strength and poor adhesive strength. Most non-destructive testing (NDT) methods can readily detect discontinuities such as void, gross unbond, porosity, etc in a joint. Some techniques can also provide correlations 
between certain properties of a joint and its cohesive strength, but there is no NDT technique that can detect poor adhesive bond strength accurately and reliably. The quality control of the adhesive bond strength at the interface between the adhesive and the adherends is usually carried out during or immediately after the surface treatment of the surfaces to be bonded [14]. Instruments which provide a measure of the adherend surface cleanliness by detecting changes in electron emission energy or surface impedance are available comercially $[15,16]$.

For the past decades, several NDT methods have been developed to detect discontinuities and to evaluate bond quality. The important NDT techniques for bond inspection are :

(a) Ultrasonics

(b) Sonic vibration

(c) Acoustic emission

(d) Radiography

(e) Thermography

(f) Optical holography

(g) Liquid penetrant

(h) Acousto-ultrasonics

In the following sections, each method will be described and its usefulness and limitations in evaluating bond quality will be discussed. The acousto-ultrasonic technique will be discussed in detail in the next chapter.

\subsection{Ultrasonics}

As the name implies, the ultrasonic techniques utilize high frequency (1-20 MHz) acoustic waves to interrogate the quality of bonded structures. Ultrasonic methods have been 
used widely due to their flexibility and effectiveness in inspecting bonded joints. Different ultrasonic techniques are available for non-destructive inspection of bond quality, namely, (i) Pulse echo, (ii) Through-transmission, (iii) Interface and plate waves, (iv) Resonance and (v) Ultrasonic spectroscopy.

\subsubsection{Pulse Echo}

The basic principle of the pulse-echo technique is shown in Figure 2.1. A repetitive acoustic pulse is transmitted into the bond through a transducer and the same transducer also receives the return signals (echoes) coming back from flaws in the bond or the interfaces of the joint which are displayed on a cathode-ray tube oscilloscope. Acoustic coupling between the transducer and the bonded joint can be achieved by direct contact through a layer of gel or grease (contact testing), or by immersing the joint in a water bath (immersion testing) or by a water jet. The latter is mostly used for inspection of large bonded structures.

In most cases, discontinuities in bonded joints can be successfully detected using the pulse-echo method [17-19]. However, there are difficulties in detecting echoes from the back layer of a multi-layered bonded structure, especially in a honeycomb structure, particularly where non-metallic cores or double honeycombs are employed $[20,21]$. The use of the pulseecho method for detection of discontinuities has been reviewed elsewhere [22-24]. Using the pulse-echo method, Alers et al [25] found a correlation between the cohesive shear strength of an aluminium lap joint and the longitudinal velocity of sound in the adhesive bond line. They also examined the relationship between the attenuation of longitudinal waves and cohesive shear strength of the bond. Rose and Meyer [18] showed a relationship between the failure load of adhesively bonded aluminium specimens and the ratio of front wall echo to bond echo. Even though the bonds were designed to fail in the adhesive failure mode, the bond line echo amplitude was shown to be governed by the bulk cohesive properties. In another study, Rose and Meyer [26] found that there was no distinguishable correlation between echo amplitude and bond strength of aluminium bonded joints at a test frequency of $10 \mathrm{MHz}$. However, when $20 \mathrm{MHz}$ was employed, a correlation was observed and the reason for this was not clear. Kline et al [27] utilized the pulse-echo technique to measure 
ultrasonic attenuation and phase velocity in adhesively bonded steel specimens. They monitored changes in the moduli of the adhesive during cure process as well as during cyclic loading.

\subsubsection{Through-Transmission}

The through-transmission method uses separate transmitting and receiving transducers positioned on either side of the joint as shown in Figure 2.2. The method requires the two transducers to be exactly aligned. The coupling between the transducers and the joint can be achieved in similar ways to those in pulse-echo method. The contact throughtransmission technique has found little practical use because of the difficulty of probe alignment and the requirement for good contact on both sides of the joint. With the immersion or water jet techniques, both probes are scanned along the bonded area and the magnitude of the transmitted signal is monitored. Scanning is often carried out in an automated C-scan system which produces a two-dimensional image presentation of flaws in the structure. The amplitude of the signal at the receiving transducer reduces when a defect is present. This method has a high level of confidence for detection of discontinuities [22]. It is particularly suited for inspection of honeycomb structures [28,29]. Cohesive bond strength of an adhesively bonded structure may also be assessed with the through-transmission methods by analysis of the amplitude of the received signals [14].

\subsubsection{Interface and Plate Waves}

Conventional ultrasonic methods use normally incident beam to inspect test components. Interface and plate waves are generated when the incident beam strikes the test material at oblique angles. In this technique, the plate and interface waves propagate along the bond line. Accordingly, it can be said that information about the interfacial bond strength might be obtained from the the velocity and attenuation of these waves.

Thompson et al [30] examined the change in cohesive strength of aluminium bonded honeycomb panels by measuring the velocity of surface waves at $300 \mathrm{kHz}$ frequency. 
They found a linear relationship between the surface wave velocity and cohesive strength. Rokhlin [31-33] investigated the application of ultrasonic surface and interface waves for evaluation of thin interface layers, in particularly, adhesively bonded structures. His arguments are that the interface waves produce shear stresses at the interface which are sensitive to variations in the adhesion quality. Also, the interface waves propagate along the interface and hence are sensitive to changes in the properties of the adhesive and of the interfacial bond between the adhesive and the adherends. Rokhlin evaluated the curing of structural adhesives by measuring the velocity of the interface wave which is related to the shear modulus of the adhesive and showed a correlation between the shear strength of the bond and the effective shear modulus which was calculated based on the velocity and the attenuation of interface waves. Kline and Hashemi [34] monitored fatigue damage development in single-lap shear aluminium specimens using interface waves, termed as guide waves. They noted that not only the amplitude of the transmitted signal decreased but also its phase velocity changed as cyclic loading was increased. Chapman [35] used a commercially available bond tester which relied on the plate waves (Lamb waves) to quantify the bond quality of fibreglass reinforced plastic bonded joints. Rokhlin [33] discussed the use of Lamb waves method to evaluate adhesive during curing, whereas Pike and Williams [36] studied the effect of cure temperature and heat-up rate on the performance of adhesively bonded honeycomb structures using the ultrasonic plate wave technique.

\subsubsection{Resonance}

In resonance methods, the frequency of a continuous acoustic wave, injected locally to a bonded joint, is changed until a resonance condition is produced. Standing waves are generated during the resonance as illustrated in Figure 2.3 for the cases of good and poor bond. At resonance frequency, the wavelength of the standing waves is twice the thickness of the joint given by:

$$
\lambda_{D}=2 D=\frac{v}{f_{D}}
$$


or

$$
f_{D}=\frac{v}{2 D}
$$

where $\lambda_{D}$ is the wavelength of the standing wave in the joint area; $D$ is the thickness of the joint; $v$ is the velocity of sound waves in the adherends (the thin adhesive layer is neglected) and $f_{D}$ is the resonant frequency. As shown in Figure 2.3, when there is a void, the resonant frequency will change sharply due to the presence of a gap which is "seen" by the transducer as change of thickness. Therefore the corresponding resonant frequency at the new thickness, $d$, is then:

$$
f_{d}=\frac{v}{2 d}
$$

One of the most widely used commercial instruments using the principles of ultrasonic resonance technique is the Fokker Bond Tester type II [37,38]. This instrument uses both resonant frequency and the amplitude of the standing waves in detecting discontinuities. In some cases, it can also predict the change in the cohesive strength of bonded joints due to variations in thickness of the substrates or the adhesive. Using the Fokker Bond Tester, Clemens [39] successfully estimated the change in the flatwise tensile strength of honeycomb sandwich panels due to variations in the foil thickness and the cell size. The application of resonance method in assessment of bond strength has also been reviewed by Curtis [23].

\subsubsection{Ultrasonic Spectroscopy}

In conventional ultrasonic testing techniques, a narrow frequency band is often used. Thus, the information obtained from the ultrasonic signals has to be deduced solely from amplitude variations. Ultrasonic spectroscopy, however, utilizes broadband ultrasonic transducers and instruments which, by analogy with visual inspection of white light, provide additional information corresponding to the colour of the inspected item. The techniques are generally carried out by using a pulse-echo method at a typical frequency range of 0.5 to $10 \mathrm{MHz}$. The echoes from the interfaces of bonded joints are then analysed in time domain (amplitude versus time) and frequency domain (amplitude versus frequency). The 
ultrasonic spectroscopy is based on the principle of constructive and destructive wave interferences to obtain information about ultrasound in the frequency domain.

Rose and Meyer [18] first reported the application of ultrasonic spectroscopy to nondestructive inspection of bond quality of aluminium bonded joints. They found that a change in the bond line thickness can be distinguished from the differences in the ultrasonic frequency spectra. When the bond line is sufficiently thick, the reflections of the ultrasonic beam from the interfaces can be resolved in the time domain provided that the ultrasonic pulses are shorter than the bond line thickness. However, when the bond line is thin, which is the usual case, the reflections generally can no longer be resolved clearly in time domain. The contribution of ultrasonic spectroscopy technique is to the generation and reception of high resolution short pulses of relatively low-frequency ultrasound which can be used to resolve the reflections from the thin bond line. Brown [40,41] demonstrated that the debond pattern in an aluminium bonded structure as well as the spacing of the honeycomb in an aircraft bonded-honeycomb structure can be clearly resolved with the wideband technique. The technique has been used to detect the quality variations of the aluminium alloy bonded joints due to fabrication processes $[42,43]$ and due to hydrothermal degradation [44]. Chang et al [45] reported a linear relationship between the bond strength in aluminium single lap-shear specimens and ultrasonic parameters obtained from the ultrasonic spectroscopy technique.

\subsection{Sonic Vibration}

There has been a wide range of sonic vibration techniques used for non-destructive inspection of adhesively bonded joints $[24,46]$. These techniques are based on the fact that a local change in stiffness due to a flaw will change the vibrational characteristics of the structure. The testing methods involve applying a vibrational force at several test points and measuring the vibration response of the structure. The typical frequency range used in these techniques is $20-30 \mathrm{kHz}$. Since the minimum void size detectable increases as the frequency decreases, the sonic vibration techniques will not be able to detect flaws as small 
as those detectable by ultrasonic techniques which operate in $\mathrm{MHz}$ range. However, the sonic vibration tests are usually faster than the ultrasonic techniques since they do not require a coupling agent between the transducer and test structure. These techniques are most sensitive to gross defects close to the surface of a stiff structure such as a disbond in a honeycomb structure. There are basically three categories of sonic vibration techniques, often referred to as coin tapping, mechanical impedance and membrane resonance.

\subsubsection{Coin Tapping}

The coin tap test is the oldest and simplest technique for inspecting the quality of bonded structures. It is still used widely in modern aviation industry due to its simplicity. In this method, The difference between good bond or disbond areas in a bonded structure is determined by listening to the change of sound produced when the structure is tapped. A common standard tapping device used in the US industry is the US 25 cent coin and the method is therefore often referred to as coin tapping. The technique is regularly used in the inspection of metal to metal bonded joints and honeycomb structures [19]. It can only detect disbond or void in the upper facing sheet to adhesive and is usually unable to detect voids at second, third or deeper adherends. Until now, the test method has remained largely subjective and furthermore, when the coin tapping test is used to inspect large bonded structures, it becomes tedious and impractical. A special device has been developed to overcome this problem [17].

\subsubsection{Mechanical Impedance}

The impedance technique has been used for almost 30 years in Soviet Union [47]. Only recently the application of this technique has increased in the west with the marketing of the Acoustic Flaw Detector and the Mechanical Impedance Analyser (MIA 3000), developed by Inspection Instruments Limited based on the original Soviet design. The technique utilizes the principle of a local mechanical impedance measurement to detect voids and 
disbonds in the plane parallel to the test surface. The point impedance, $Z$, is given by [47]:

$$
Z=\frac{F}{v}
$$

where $F$ is the harmonic force input to the structure and $v$ is the resultant velocity of the structure at the point of application of the force. The measurements with the commercially available instruments are generally carried out at a single frequency, typically between 1 to $10 \mathrm{kHz}$. Cawley $[47,48]$ showed that the impedance of a good bond area was generally higher than that of a disbond area below a frequency of $12 \mathrm{kHz}$. The fact that impedance decreases as the probe moves from a good to a defective area can be used to detect voids and delaminations. The technique is most sensitive when the voids are close to the test surface and the base structure is relatively stiff. Cawley [49] also reported the use of the mechanical impedance method to produce C- scan presentations of defects in carbon fibre reinforced plastic bonded honeycomb panels with deliberately introduced defects.

\subsubsection{Membrane Resonance}

The membrane resonance techniques are based on the principle that a planar void can be modelled as a plate restrained around the edges by the surrounding structure. This plate can resonate, the first mode being the membrane resonance and having a deflected shape similar to that of a diaphragm. As the frequency of the vibrational excitation approaches the membrane resonant frequency, the mechanical impedance measured over the defective area decreases to a minimum and the response amplitude for a given force input increases. Thus, at or close to membrane resonant frequency, the response amplitude of a defective area will be much greater than that of a sound region of the structure. Hence, a non-destructive test on bonded structures can be based on response measurements alone, assuming the input force to be approximately constant. The technique is most sensitive when the excitation is applied at membrane resonant frequency, but since frequency is dependent on flaw size and depth [46], wideband frequencies should be used. The Fokker Bond Tester Type I (which is different from the Fokker Bond Tester type II, explained in Section 2.1.4) uses this principle with wideband excitation covering a frequency range 
of 0.5 to $10 \mathrm{kHz}$ [37]. The technique can also be used with a single excitation frequency even though the sensitivity to defects will be reduced as the excitation frequency deriates from the membrane resonant frequencies of the defects. The Harmonic Bond Tester developed by Boeing from the Eddy Sonic Test System excites the test structure at a single frequency by inducing eddy current and measuring the materials response with a microphone placed within the eddy current coil $[50,51]$.

\subsection{Acoustic Emission}

Application of stress to a material beyond the yield point will eventually lead to dislocation, permanent deformation or crack growth. This internal response is usually associated with a local release of energy, part of which propagates as stress waves, known as acoustic emission. Since in many materials, acoustic emission is generated at stress levels lower than the fracture stress, it can be used to detect subcritical failure process in materials. Hence, if sufficient emissions are generated from the bonded joint at an acceptably low applied load, then acoustic emission technique has the basic requirements of a quantitative non-destructive test to characterize the joint. A typical acoustic emission test set-up for a bonded joint is illustrated in Figure 2.4.

The early attempt to apply acoustic emission technique to adhesively bonded structures was carried out by Schmitz and Frank [52]. They found a correlation between the density of emissions at frequency above $16 \mathrm{kHz}$ and strength of adhesively bonded aluminium honeycomb specimens. Another early work on acoustic emission for bonded joint is that of Beal [53] who used the acoustic emission technique to predict the ultimate strength of bonded joint specimens and then compared this with the actual breaking stress. The maximum error in the strength prediction was found to be about $30 \%$. Using a similar technique, Pollock [54] reported that the accuracy of the fracture stress prediction increased as the load approached the failure load during preloading. Curtis and Speake $[55,56]$ developed a broadband (0.01-2 $\mathrm{MHz}$ ) acoustic emission spectrum and energy analysis system to be used in studying the fracture of aluminium bonded joints loaded in tension. An empirical 
relationship was established between the cumulative acoustic emission energy at fracture per unit volume of adhesive and the ultimate shear strength of the joint. The latter was varied by changing the glue line thickness. The use of acoustic emission technique to study the effect of elevated temperatures on adhesive joints was carried out by Hill [5i]. The acoustic emission study of the effect of aging bonded joints in a warm and moist environment was reported by Brockmann and Fischer [58]. Most of these studies indicate that generally weak joints emit more acoustic emission during loading than strong joints.

\subsection{Radiography}

The fact that radiography can detect density variations by means of attenuation of rays passing through the material can be used to locate defects in bonded structures. The success of using radiography depends upon the ability to distinguish small changes in the absorption of X-rays which is determined largely by the density of the material. Generally, $\mathrm{X}$-radiography cannot be used for metal to metal bonded joints because the adhesive layer usually has a much lower density than that of the metallic adherends unless the adhesive contains metallic fillers or is X- ray opaque such as AF55 and FM400 [19]. However, radiography can be readily used for detecting voids in joints with fibre- reinforced plastic adherends because the densities of adherends and adhesive are generally of the same order [22]. X-radiography is also useful for inspecting honeycomb structures to detect water intrusion into honeycomb cells, crushed core and other defects $[19,50,59,60]$.

Neutron radiography is based on the absorption or scattering of neutrons by hydrogenous materials such as hydrocarbons which are generally present in adhesives. With the use of neutron radiography, a thin adhesive layer can be detected even through thick layers of metallic adherends [19,22]. Unfortunately, for the time being neutron sources which are typically nuclear reactors and accelerators are somewhat limited. 


\subsection{Thermography}

The application of thermography for inspection of bonded structures is based on variation in thermal conductivity, thermal emissivity or thermal capacity. All of these thermal properties are related to the density and the thickness of the adhesive layer. Thus, variation in the bond quality can be detected from differences in temperature during heating or cooling of the structure. There are a number of thermal inspection methods for adhesively bonded structures. One method is to heat the surface of the bonded assembly and detect temperature variations on the same side. In this case, disbond areas which resist transfer of heat, will be hotter than well bonded areas. Another method is to heat the component from the back surface and measure temperature differences at the front surface. In this case, the defective areas will be cooler due to the poor heat conduction through voids. An alternative method is to excite the structure at one of its resonant frequencies such that defective areas, due to frictional heating, will show a local rise in temperature [61]. Temperature sensing is normally done with a scanning infra-red camera and recently, the use of video recording techniques has considerably simplified the process of recording [62]. Some works on thermographic testing of aluminium to aluminium and aluminium to foam adhesively bonded structures [63] as well as honeycomb structures [50] have been reported. The use of temperature sensitive coatings such as liquid crystals and thermolumiscent coatings may also be used for the detection of temperature patterns on bonded panels $[14,50]$.

\subsection{Optical Holography}

Holography interferometry has the capability to measure changes of surface displacement in the order of half a wavelength which in the optical case, is less than $0.5 \mu \mathrm{m}$ [22]. To obtain such a displacement in an outer adherend, load is applied to a bonded structure by vibration, pressure or heat. Defective areas show as local perturbations in the holographic interferogram. The technique is useful for inspection of sandwich structures, but not for lap joints. The application of this technique for inspection honeycomb structures have been 
discussed by Schliekelmann [14], Hagemaier [17] and Kersch [64]. Holographic techniques can generally detect voids, delaminations, inclusions, water damage, and crushed core in honeycomb structures.

\subsection{Liquid Penetrants}

This technique can only be used when the flaw is open to the surface. This type of flaw does not often occur in bonded joints. However, Crane [65] has developed a relatively new penetrant system that proves to be useful in aircraft industry. The penetrant only becomes fluorescent when it comes in contact with metal substructure inside a honeycomb structure with composite skins or in an adhesively bonded structure with metallic adherends. This penetrant system would provide indications of the presence of the openings which would permit water entry and thus corrosion development.

\subsection{Comparison of NDT Methods for Bond Quality Inspection}

As discussed in previous sections, several nondestructive testing (NDT) techniques can be used for inspection of bond quality and most of these NDT methods are able to detect voids and disbonds in bonded structures. The ultrasonic techniques have been considered to be best suited for locating subtle defects such as a small void, bond line porosity and unbond. The acoustical mismatch of thin air layer or small air bubbles is sufficient to prevent the ultrasonic energy from penetrating the joint and therefore makes the unbond or porosity indication clear. In terms of the overall detection ability of the ultrasonic methods investigated in the Primary Adhesively Bonded Structure Technology (PABST) program $[19,22]$, immersion C-scan through transmission and pulse echo techniques have been rated highly for bond quality inspection of laminates. According to the work by Segal et al [66], when the unbond radius, $a$, is smaller than the transducer radius, $r$, the pulse echo technique is more accurate than the through transmission technique. On the other 
hand, in the case of an unbond situation when $a / r \geq 1$, both techniques are comparable in accuracy, but through transmission is usually preferable in this case because its cathode ray tube (CRT) presentation of the unbond is more easily followed especially for thin substrates with echoes of the ultrasonic beam from interfaces which are difficult to resolve.

The ultrasonic immersion techniques, although considered to be the most capable in detecting small defects, are not suitable for field inspection. These techniques are, however, particularly useful for inspecting components during production. For in-service inspection, the ultrasonic contact techniques or water jet probes can be used. To detect large defects in the adhesive layer, sonic vibration techniques are more practical because coupling agents are not required. Radiography is more effective to inspect complex geometry joints and honeycomb structures, but X-radiography has found little use for metal to metal joints in contrast to its use on fibre-reinforced plastic joints and honeycomb structures. Neutron radiography, however, can be used for bonded structures with metallic substrates. Similar to radiographic techniques, thermography and optical holography are more useful for inspection of honeycomb structures, but these techniques still need further developments in order to be used effectively for bonded structures and are costly to implement.

A number of studies has been carried out to predict cohesive bond strength, but yet the problem has not been solved satisfactorily. Cohesive bond strength depends on many variables such as bond line thickness, curing condition, porosity of bond line, and environmental effects. Fokker Bond Tester type II, a commercially available instrument, which employs the principle of resonance has been successful in predicting the cohesive bond strength of the bonded structures with varied glue line thickness and with flaws in the adhesive. It is, however, unable to determine the variation in cohesive strength due to other variables. Other ultrasonic methods such as ultrasonic spectroscopy and pulse echo using the velocity and attenuation of the signals can also be used to predict cohesive strength. Ultrasonic methods employing interface or plate waves are regarded to be more effective in evaluating the cohesive properties of the adhesive layer since the ultrasonic interface or plate waves propagate along the bond line.

Currently, there is no applied method that is capable of predicting reliably the adhesive 
bond strength of a bonded structure after it has been assembled. Ultrasonic methods utilizing interface or plate waves show some promise. The techniques are still in the research and development stage, and are yet to be established. Acoustic emission technique generally is able to evaluate the integrity of a bonded joint as a whole. However, whether this technique is truly nondestructive testing techniques is still a question because such emissions can only be generated when the joint undergoes an internal response induced by local stresses.

\subsection{Conclusions}

Gross defects in bonded structures can be easily detected with the available NDT methods. No single method is universally applicable for detection of different types of defects in adhesively bonded joints. Each technique has its own advantages and disadvantages under certain circumstances. The problem of estimating the cohesive bond strength is only partially solved, but continuing research in this area has shown considerable promise. The problem of estimating the strength at the adhesive- adherend interface or even detecting poor adhesion due to factors such as improper substrate surface preparation still remains unresolved.

Of all the NDT methods available, ultrasonic techniques tend to be more versatile and quantitative. Acoustic emission techniques, on the other hand, are more appropriate for evaluation of the integrity of a bonded joint as a whole, but because the structure has to be stressed to produce emissions from subtle flaw growth, an alternative or improvement to the acoustic emission techniques is required. 


\section{Chapter 3}

\section{ACOUSTO-ULTRASONIC TECHNIQUE}

\subsection{Introduction}

As discussed in Chapter 2, acoustic emission (AE) and ultrasonic techniques have been used extensively in evaluating the integrity of structures, investigating the properties of materials and detecting flaws. However, in many practical situations, the application of acoustic emission techniques to adhesively bonded joints is not recommended since the joints must be subjected to mechanical loading to create emissions and such loading can result in permanent damage to the bonded joints. Although conventional ultrasonic techniques, which often employ an incident beam normal to the plane of the bond, can detect defects such as voids and disbonds, they are not very sensitive to the properties of the bond line which constitutes a small fraction of the total beam path length. The acousto-ultrasonic ( $\mathrm{AU})$ technique provides an alternative approach to acoustic emission and ultrasonic methods in the characterization of adhesively bonded joints.

Recent laboratory studies demonstrated that the acousto- ultrasonic technique can be used to provide quantitative information about the mechanical performance of bonded joint specimens by correlating the AU stress wave propagation characteristics with the joint strength. Williams et al [67] used the technique to evaluate the strength of bonded joints 
in automotive glass fibre composites, while Kapoor and Prakash [68] applied the technique to evaluate the strength of bonded joints in aluminium. Dos Reis et al [69.70] showed a correlation between the peel strength for rubber sheets bonded to steel specimens and stress wave factor, which is a parameter measurable by the acousto-ultrasonic technique. There are numerous examples of successful applications that show the feasibility of the acousto- ultrasonic technique as a means of estimating mechanical strength variations in structural composites [4-9] as well as estimating residual strength and degradation from cyclic fatigue and impact damage $[10,11,71,72]$. The technique has also been used to evaluate wire rope strength [13], nylon rope tension [73], filler content in wood and paper product [74], strength of ceramic materials $[75,76]$, the effect of hydrothermal aging on composites $[77,78]$ and porous metal diffusion bond quality [79]. It has been reported that the acousto-ultrasonic technique can also be used to detect delaminations, hidden impact damage areas or other overt flaws [12].

Besides providing quantitative information in the characterization of materials, the acousto-ultrasonic technique also offers some practical advantages. The technique requires access to only one side of the structure which makes it effective to be used on existing in-service materials where two-side accessability and ability to disassemble the structure are not possible. Another advantageous aspect of the acousto-ultrasonic technique relative to the conventional ultrasonic techniques is its ability to overcome the high attenuation, common to most composite structures, because the acoustic emission sensor used as the $\mathrm{AU}$ receiving transducer provides the necessary sensitivity. In this chapter, the basic principles of the acousto-ultrasonic technique in the characterization of adhesively bonded joints are described. Factors that may affect the measurement of the acousto-ultrasonic data are examined and discussed.

\subsection{Acousto-Ultrasonic Principles}

In the acousto-ultrasonic technique, shown schematically in Figure 3.1, a broadband transducer is employed to transmit a repetitive series of ultrasonic pulses into the test specimen. 
A receiving $\mathrm{AE}$ transducer is placed on the same side of the specimen at a specific distance from the transmitting transducer to intercept the propagating stress waves resulting from the injected ultrasonic pulses. Although the transmitting transducer injects longitudinal waves normal to the joint specimen surface, the sound waves radiated into the material will produce oblique reflections and shear waves [80]. The resultant stress waves which consist of longitudinal and transverse components then undergo multiple reflections at the boundary surfaces of the specimen and interact with a significant fraction of the bond line volume that lies in their path. Thus, they can be affected by those microstructural and morphological properties which also determine structural performance. In many situations, it is possible to obtain information on mechanical behaviour from stress wave propagation data.

Stress wave propagation can be related to several parameters that are measurable from an acousto-ultrasonic waveform in time or frequency domains. The important parameters in time domain include signal amplitude, ring-down counts, duration and root-mean-square (RMS) amplitude (see Figure 3.2 for a definition of these parameters). Vary [4] defined another parameter known as stress wave factor (SWF) which is given by the expression :

$$
\mathrm{SWF}=R G C
$$

where $R$ is the repetition rate of input pulses; $G$ is a pre-determined time interval; and $C$ is number of oscillations in the received waveforms which exceed a preselected voltage threshold (Ring-Down Count). According to Vary [80], the stress wave factor may also be defined in many other ways. The definition given in Equation 3.1 is based directly on acoustic emission practice. The formulation of a definition for the stress wave factor depends on which features in a waveform are most relevant to a given probe configuration, material, or structure geometry. Williams and Lampert [10] used a somewhat different approach in calculating the magnitude of the stress wave factor by summing the amplitudes of the oscillations in the received waveform. In the present work, this parameter will be designated as the "acousto- ultrasonic parameter" or AUP to distinguish it from the stress wave factor (SWF). Since the AUP takes into account both ring- down counts and the amplitude of the signal, it may be more sensitive to material properties than the 
conventional SWF. For this reason, the AUP was used as the preferred acousto-ultrasonic parameter in the present work.

Based on the work of Williams and Lampert [10], the following procedure was developed to compute the value of the AUP from the acousto-ultrasonic signal. First, the noise level was determined and a threshold level was set just above the noise $\left(V_{0}\right)$. Then the total number of counts above $V_{0}$ was determined, the threshold was increased by a small interval $\Delta V$ and the number of counts above the new threshold was found. The difference of the two counts was obtained and multiplied by the amplitude at that threshold level. This process was repeated until the threshold was equal to or greater than the peak amplitude of the waveform. This procedure can be expressed by [81]:

$$
\text { AUP }=\sum_{i=0}^{p} V_{i}\left(C_{i}-C_{t+1}\right)
$$

where $V_{i}$ and $C_{1}$ are the threshold and number of counts at the $i$ th level respectively and $V_{p}$ corresponds to the peak amplitude of the waveform. This procedure is illustrated schematically in Figure 3.3.

Vary $[4,80]$ described the stress wave factor as a relative measure of stress wave energy transmission. His hypothesis was that the decrease in stress wave energy flow corresponds to the decrease in fracture resistance of materials. Since AUP is a parameter defining the stress wave factor, its measurement provides a means of rating the efficiency of stress wave energy transmission for a given joint specimen. If the joint specimen demonstrates a more efficient stress wave energy transmission (higher AUP value), then it will have higher joint strength. Since the AUP provides a relative measurement, it is usually better to normalize the AUP value for comparison with the strength variations of the joint specimens. It has been found practical to normalize the AUP values with respect to the maximum AUP value obtained for these quantities.

\subsection{Factors Affecting Acousto-Ultrasonic Data}

The results of acousto-ultrasonic measurements are affected by several factors which are related either to the transducer-specimen attachment configuration or to the settings and 
characteristics of the transmitting and receiving instruments. The important factors in transducer-specimen attachment are :

(a) the applied pressure on the transducers

(b) the type and amount of couplant

(c) the orientation of transducers

(d) the spacing between the transmitting and receiving transducers

(e) the location of transducers on the specimen.

A series of experiments was conducted in order to optimize the AU measurement system with respect to the above factors and the results are described in the following.

It has been reported [7] that for repeatable AU measurements, large loads (as much as 20 pounds) should be applied to the transducers. In our studies, we found that it was not necessary to apply a large load but it was important that the load be applied uniformly and with sufficient magnitude in order to eliminate unwanted reverberations within the couplant. A specially designed fixture (Figure 3.4 ) with a spring loading arrangement was found to be satisfactory in terms of the magnitude and uniformity of the applied pressure required to achieve repeatable $\mathrm{AU}$ data. In addition to the applied pressure, the type of coupling fluid used between the transducer and the specimen is also important. Among several couplants including water, motor oil, vacuum grease, and commercial coupling agents such as AET SC-6 and Ultragel II examined, the latter was found to produce the most reproducible data and was also the best in terms of wetting ability as well as simplicity of application and removal. The Ultragel II couplant has the fastest wetting ability as can be illustrated in Figure 3.5 which shows that early stabilization of the AU measurement with time was achieved when the Ultragel II was used as couplant. Also shown in the Figure, the Ultragel II couplant generally provides the highest value of AUP for a given joint specimen, thus indicating highest ultrasonic transmission. Other factors such as transducer orientation, distance and location were also studied and the optimum conditions were determined and implemented in this study. The overall repeatability of 
AU data using the test fixture was assessed and found to be satisfactory as indicated by the standard deviation of eight different trials on a reference specimen reported in Table 3.1 .

The next step in the system optimization process was to find the best combination of transmitting frequency and amplitude gain. The system gain was selected on the basis of the sensitivity of the transmitting and receiving transducers and set at a level such that the signal to noise ratio was sufficiently high while the maximum signal amplitude was held below the saturation level of the receiving instruments. The gain was held constant for all measurements. The frequency spectrum of the input pulse and the frequency response of the transducers determine the ultrasonic wavelength in the material. The relationship is expressed by :

$$
\lambda_{D}=\frac{v}{f}
$$

where

$$
\begin{aligned}
& \lambda_{D}=\text { wavelength } \\
& v=\text { wave velocity } \\
& f=\text { frequency. }
\end{aligned}
$$

Vary [4] has reported that optimum conditions for AU measurement occur when the wavelength is integrally related to the specimen thickness $(D)$ such that resonance effects can occur, i.e.,

$$
D=n \lambda_{D}
$$

where $n$ is an integer. From Equations 3.3 and 3.4, one should be able to estimate the resonant frequency of the test material, provided that the acoustic velocity in the material is known. Figure 3.6 shows a typical waveform with resonant conditions which was measured from an adhesively bonded joint in graphite/ epoxy composite specimen. The individual wavefronts of the AU waveform shown in the Figure are in phase and there is no overlapping between them. Figure 3.7 shows a typical waveform with off-resonant conditions which was measured from the same specimen but with different transducers which had the frequency response that did not satisfy Equation 3.4. As can be seen in the Figure there were overlappings between the individual wavefronts. 


\subsection{Conclusions}

Acousto-ultrasonic technique combines beneficial aspects of acoustic emission and ultrasonic technology. It also offers practical advantages such as one-side accessibility and overcoming high attenuation problem.

The conventional stress wave factor (SWF) defined by Vary [4] does not take into account the amplitude of oscillations of waveforms. A modified stress wave factor which takes into account both ring-down counts and amplitude of the signals was developed based on a concept outlined by Williams and Lampert [10]. This factor was named as the acousto-ultrasonic parameter (AUP) and was found to be a sensitive indicator.

For repeatable acousto-ultrasonic measurements, the test conditions and configuration such as the pressure applied on the transducers, the coupling medium, the transducer spacing, orientation and location should be carefully selected and optimized. Other important factors such as type of transducers and input signals may also affect the AU measurement and should be considered. These factors are dependent on the material and geometry of the test piece. For example, to obtain a resonant condition in the test specimen, appropriate transducers and input signals in terms of centre frequency and bandwidth are required for a particular test material and geometry. All of these test conditions should be kept constant during the $\mathrm{AU}$ measurements so that comparison of the mechanical performance between test specimens can be made. 


\section{Chapter 4}

\section{FACTORS AFFECTING JOINT STRENGTH}

\subsection{Introduction}

Before attempting to evaluate the performance of an adhesively bonded joint using acoustoultrasonic technique, it is necessary to examine the factors that may affect the joint strength measured from the destructive testing. Besides improper bonding or defects that may occur in the adhesive, other important factors that may affect the strength of an adhesively bonded joint include the joint configuration, the joint dimensions and the properties of the adhesive and the adherends.

Figure 4.1 shows some typical joint configurations which are commonly encountered in current engineering practices. Most of the joint configurations are designed to transfer load in shear. Of all the various joint configurations and methods of loading, the single lap joint loaded in tension has received the most attention from researchers in adhesive joints for two reasons. First, the single lap joint is one of the joint designs most commonly used in industry. Secondly, it is simple and convenient test configuration for evaluating adhesive joints, as may be seen from its common use in the standard test methods (for example, ASTM D1002-72). For these reasons, the single lap joint configuration is selected for this study. 
The strength of a given type of joint for a given type of load depends on the stress distribution within the joint. This stress distribution depends on the joint geometry, as illustrated in Figure 4.2, and the mechanical properties of the adhesive and the adherends such as their individual strengths and elastic moduli, and the adhesive stress-strain properties. The stress distribution within a joint can generally be determined by solving a boundary value problem through establishing a series of differential equations to describe the state of stress and strain in the joint. In the simple case where the adhesive and adherends are all elastic, it may be possible to solve the differential equations for a given boundary conditions. These methods become increasingly more difficult to use as nonlinearities arise. However, it is necessary to consider the non-linear adhesive behaviour if the joint strength is to be predicted, especially for the structural adhesives which may behave as elastic-plastic materials.

A considerable effort in solving the stress distribution and in predicting the strength of the joint has been undertaken by researchers in this field. A review on the theoretical analyses of the stress distribution and the strength of adhesively bonded joints has been published by Matthews et al [82]. In this chapter, studies on the elastic analysis of the stress distribution within a joint are briefly reviewed. Then, by using one of the analytical solutions, a parametric study is carried out to examine the effect of joint dimensions on

the stress distribution in the elastic region. Finally, the elastic- plastic situation when the adhesive becomes plastic is considered where a parametric study on the effect of joint dimensions upon the joint strength is attempted.

\subsection{Linear Elastic Analysis}

\subsubsection{Volkersen's Analysis}

The earliest theoretical stress analysis in a single lap joint was presented by Volkersen [83], the so-called shear lag analysis. In this analysis, it is assumed that the adhesive deforms only in shear, while the adherends deform only in tension and that there are no peel stresses attributed by the bending of the adherends. By setting up the differential equations of 
linear elasticity, Volkersen showed that the shear stress $\tau$ at any position $x$ along the overlap of a joint (Figure 4.2) is given by :

$$
\tau=\frac{T \delta \cosh \{\delta(x / l)\}}{2 l \sinh \delta}
$$

where

$$
\delta=l \sqrt{\frac{2 G_{a}}{E t h}}
$$

$T=$ Load per joint width

$G_{a}=$ Shear modulus of the adhesive layer

$E=$ Young's modulus of the adherends

Volkersen's analysis is unsatisfactory for two reasons. First, there is a bending moment applied to the joint in addition to the in-plane tension due to the noncollinearity of the two forces $T$ shown in Figure 4.2. Secondly, As the applied load increases, the adherends bend, allowing the joint to rotate. The rotation subsequently alters the direction of the load line in the region of the overlap, thus giving rise to a geometrically non-linear problem because the joint displacements are no longer proportional to the applied load.

\subsubsection{Goland and Reissner's Analysis}

An earlier analysis taking bending moment and peel stresses into account was carried out by Goland and Reissner [84] who considered two cases in their analysis. In the first case, it is assumed that the glue line is extremely thin (Figure 4.2 where $h$ is assumed approaching zero) and that both the adhesive and the adherends have the same young's moduli. Thus, the deformation of the adhesive is negligible in the stress analysis.

In the second theoretical approximation of Goland and Reissner, it is assumed that the bond line is thin, but its deformation is crucial to the stress distribution in the adhesive. In this case, they assumed that the adhesive layer is much less rigid than the adherends so that the transverse normal strain and the shear strain in the adherends are negligible compared with the corresponding strains in the adhesive. Also, they treated the adherends as cylindrically bent plates and the adhesive behaving like a system of infinitesimal springs placed between the two plates. With these assumptions, they derived the shear stress and 
peel stress distribution in the adhesive given by Equations A.1 and A.2, respectively in Appendix A.

They established the quantitative limits for the assumptions they made based on strain energy considerations, but later work [85] suggested that the limits were somewhat conservative. Sancaktar and Lawry [86] have recently examined the elastic tensile stresses using a photoelastic technique and reported a good agreement with the first theoretical predictions of Goland and Reissner. Ishai et al [87] confirmed that for joints which behave elastically, the second analytical solutions of Goland and Reissner were in good agreement with the experimental results for both the shear and tensile stress distributions in the adhesive layer.

\subsubsection{Later Work on Elastic Analysis}

The pioneering work of Volkersen and Goland-Reissner had clearly established the basic stress distribution in a single lap joint with elastic adherends and adhesive. However, in these early analyses, the following assumptions were made : (i) the shear and peel stresses were assumed to be constant across the thickness of the adhesive layer; (ii) the shear strains in the adherends are neglected; (iii) the longitudinal (in-plane) tensile stresses in the adhesive layer cannot always be deduced; (iv) the shear stress was a maximum, and not zero, at the overlap ends. Since the end face of the adhesive is a free surface, there can be no shear stress on it; ( $v$ ) the effect of the presence of adhesive spew fillets are ignored.

Recently, several authors [88-92], using closed-form analytical or finite element analysis, have subsequently developed elastic analyses which consider one or several of the above factors. However, Kinloch [93] reported that even with the assumptions mentioned above, the analytical solutions of Goland and Reissner [84] are relatively accurate. Cooper and Sawyer [94] also reported that based on the comparison with finite-element solutions, the closed-form analysis of Goland and Reissner was sufficiently accurate to be used in qualitative evaluation of the effects of the parametric variations on the shear and peel stresses along the mid-plane of the adhesive bond line. In using the theoretical analysis of Goland-Reissner, however, Cooper and Sawyer made slight modifications by assuming 
consistent plate behaviour throughout the derivation and by retaining small terms formerly neglected in Goland-Reissner analysis. With the modifications, they then obtained the modified shear and peel stress distributions in the adhesive along the bond line which are given by Equations A.13 and A.14, respectively.

In their analysis, Cooper and Sawyer [94] also indicated that the closed-form solution did not satisfy the adhesive free- edge shear boundary condition (zero shear stress at the free edges), but the influence of the free edge was confined to a small region and had little effect on the computed value of the shear or peel stress occuring in another larger part of the overlap region. In addition, by examining the finite-element solutions to the adhesive stress distributions, they found that the assumption of constant shear and peel stresses throughout the adhesive thickness was valid over the interior of the lap region but deteriorated in the vicinity of the free edges.

In the present work, the closed-form solutions of Cooper and Sawyer (Equations A.13 and A.14) will be used to examine the influence of the geometric variations on the adhesive stress distributions of adhesively bonded specimens used in this study in the elastic region. The properties of the materials used in these analyses are given in Table 4.1.

\subsubsection{Effect of Parametric Variations on Stress Distributions}

The single lap joint configuration used throughout this study is adopted from the ASTM D1002-72 test specimen used to obtain shear strength properties by tension loading. The geometry of the joint is shown in Figure 4.2 with a width , $b$, of $2.54 \mathrm{~cm}$. The materials that are mainly used for joints in this study are mild steel or unidirectional $\left(0^{\circ}\right)$ carbon

fibre reinforced plastic (CFRP) for adherends and modified-epoxy (FM300) for adhesive. The properties of these materials are given in Table 4.1.

According to Hart-Smith [98], it is necessary to introduce the bending stiffness parameter,$k_{b}$, in the bending stiffness, $D$, (Equation A.20) for the filamentary composite materials in the theoretical analysis such that it becomes

$$
D=k_{b} \frac{E t^{3}}{12\left(1-\mu^{2}\right)}
$$


For all $0^{\circ}$ fibres uniformly dispersed throughout the cross sectional area of the adherend. which is the case in this study, the value of $k_{b}$ is unity $\left(k_{b}=1\right)$ which is same with that for homogeneous metal adherends [98].

Using Equations A.13 and A.14, the computed adhesive nondimensional shear and peel stress distributions of steel lap joints for various loads are given in Figures 4.3 and 4.4, respectively. Using the same equations, the adhesive nondimensional shear and peel stress distributions of unidirectional CFRP lap joint can also be obtained as shown in Figures 4.j and 4.6, respectively. As can be seen from Figures 4.3-4.6, both the shear and peel distributions of steel and CFRP single lap joints used in this study are quite similar because the stiffness of the steel adherends is of the same order with the longitudinal stiffness of the CFRP adherends. As the applied load is increased, the shear and peel stress concentration factors (maximum $\tau / \tau_{a v}$ and $\sigma / \sigma_{a p p}$ in Equations A.13 and A.14) appear to decrease slightly and the overall stress distributions do not vary significantly with load.

Figures 4.7 and 4.8 show the change in the stress distributions due to variation in the adhesive thickness. Both shear and peel stress concentration factors show decreasing trend with increasing adhesive thickness. On the other hand, as the adherend thickness increases, the shear stress concentration factor decreases whereas the peel stress concentration factor increases as illustrated in Figures 4.9 and 4.10, respectively. Similarly, increasing the overlap length will increase the shear stress concentration factor, but will reduce the peel stress concentration factor as depicted in Figures 4.11 and 4.12, respectively.

\subsection{Elastic-Plastic Analysis}

The analysis discussed above considers the adherends and adhesive to behave as elastic materials, but most of the adhesives commonly used, especially structural adhesives such as modified-epoxy, exhibit elastic-plastic behaviour. Also, according to Matthews et al [82], if joint strength is to be predicted, nonlinear behaviour of adhesive should be accounted for. Several groups of researchers [98-101] have recently sought to include this behaviour in their analyses. The most extensive studies on the elastic-plastic analysis of a single lap 
joint have been carried out by Hart-Smith [98] who used closed-form analytical solutions and developed a computer program for iterative solutions. In this study, the work and computer program of Hart-Smith (see Appendix B) will be used to examine the change in joint strength due to variation in the geometry of the joint. The computer program is based on the analytical solutions of Hart-Smith to be discussed in the next subsection.

\subsubsection{Hart-Smith's Analysis}

Based on the work of Goland and Reissner [84], Hart-Smith first developed an equation for adhesive shear stress distribution in the perfectly elastic region, $\tau$, given by :

$$
\tau=A_{1} \cosh \left(2 \lambda_{2} x\right)+B_{1} \sinh \left(2 \lambda_{2} x\right)+C_{1}
$$

where

$$
\left(\lambda_{2}\right)^{2}=\left\{\frac{1+3\left(1-\mu^{2}\right) / k_{b}}{2}\right\} \frac{G_{a}}{E t h}
$$

$\mu=$ Poisson's ratio of the adherends

and $A_{1}, B_{1}$, and $C_{1}$ are integration constants. Using appropriate boundary conditions, the integration constants are found to be :

$$
A_{1}=\frac{G_{a}}{E t h} \frac{1}{2 \lambda_{2} \sinh \left(2 \lambda_{2} l\right)}\left\{T+\frac{6\left(1-\mu^{2}\right) M_{o}}{k_{b} t}\right\}
$$

where $M_{o}$ is the bending moment per unit width in the continuous adherend at the end of the joint, which can be expressed by :

$$
M_{\circ}=k_{2} T\left(\frac{t+h}{2}\right)
$$

where $k_{2}$ is bending moment factor expressed as :

$$
\begin{aligned}
k_{2} & =\frac{1}{1+\xi l+\frac{\xi^{2} l^{2}}{6}} \\
\xi & =\sqrt{\frac{T}{D}}
\end{aligned}
$$

For a single lap joint with identical adherends,

$$
\begin{aligned}
& B_{1}=0 \\
& C_{1}=\frac{1}{2 l}\left\{T-\frac{A_{1}}{\lambda_{2}} \sinh \left(2 \lambda_{2} l\right)\right\}
\end{aligned}
$$


From Equation 4.4. the ratio of average shear stress. $\left(\tau_{32}=I\right.$ 2l) to maximum shear stress along the overlap. $i_{22 x}$ can be found to be:

$$
\frac{\tau_{12}}{\tau_{m a x}}=\frac{1}{1+\left\{1+\frac{3 k_{2}\left(1-\mu^{2}\right)}{k_{b}}\left(1+\frac{h}{t}\right)\right\}\left\{\frac{\left(G_{2} /(E t h)\right)^{2}}{4\left(t_{2}\right)^{2}}\right\}\left\{\frac{2 t_{2} i}{\tanh \left(2 t_{2} i\right)}-1\right\}}
$$

which. for isotropic adherends. reduces to the solution obtained by Goland and Reissner 84 '.

Hart-Smith then extended the analysis to include the plastic region and found that the relation between the plastic $\left(\hat{\gamma}_{F}\right)$ and elastic $\left(\hat{i}_{e}\right)$ adhesive shear strains is given by :

$$
2\left(\frac{\hat{i p}_{i}}{\hat{i e}_{e}}\right)=K\left\{\left[2 \lambda_{2}\left(1-\frac{d}{2}\right)+\tanh \left(\lambda_{2} d\right)\right]^{2}-\tanh ^{2}\left(\lambda_{2} d\right)\right\}
$$

and the average adhesive shear stress $\left(\tau_{a t}=T / 2 l\right)$. in terms of plastic adhesive shear stress. $i_{p}$ is given by :

$$
\frac{\tau_{2 v}}{\tau_{p}}\left(2 \lambda_{2} l\right)=2 \lambda_{2}\left(1-\frac{d}{2}\right)+\tanh \left(\lambda_{2} d\right)+\left(1-K^{-}\right)\left\{\lambda_{2} d-\tanh \left(\lambda_{2} d\right)\right\}
$$

where

$$
F^{*}=\frac{A_{1} \cosh \left(\lambda_{2} d\right)}{\tau_{p}}
$$

and $d$ is the length of the elastic zone in adhesive bond. A computer program is required to obtain numerical solutions for Equations 4.11 and 4.12 by eliminating $K^{\prime}$ and $d$.

Hart-Smith also solved for the maximum adherend bending stress adjacent to the bond line at the end of the overlap due to the eccentricity in the load path of a single lap joint which induces high bending moment in the adherends. The maximum adherend bending stress. $\sigma_{\max }$ is given by :

$$
\sigma_{\max }=\sigma_{a 2}\left\{1+3 k_{2}\left(1+\frac{h}{t}\right)\right\}
$$

where $\sigma_{a v}=$ average normal stress $=\frac{T}{t}$

$$
\begin{aligned}
k_{2} & =\frac{1}{1+\xi l+\frac{\xi^{2} l^{2}}{6}} \\
\xi^{2} & =\frac{T}{D} \\
& =\frac{\sigma_{a l}}{\sigma_{\max }}\left\{\frac{12\left(1-\mu^{2}\right) \sigma_{\max }}{k_{b} E t^{2}}\right\}
\end{aligned}
$$


Thus, for a given allowable adherend bending stress, $\sigma_{m a x}$, the associated average stress, $\sigma_{a v}$, must be determined through iteration using the computer program shown in Appendix B.

For single lap joints, peel stresses are significant and cannot be ignored, especially at the ends of the overlap. Therefore, to accompany his elastic-plastic analysis of the shear stresses, Hart-Smith derived solutions for these peel stresses, but assumed only elastic behaviour of the adhesive since this was considered to be sufficiently accurate to model the adhesive behaviour in peel. The maximum peel stress, $\sigma_{y(\max )}$, for single lap joints with identical adherends is given by :

$$
\sigma_{y(\max )}=\frac{E_{a} k_{2}}{2 h D \chi^{2}} T\left(\frac{t+h}{2}\right)
$$

where

$$
\chi=\sqrt[4]{\frac{6 E_{a}\left(1-\mu^{2}\right)}{E t^{3} h k_{b}}}
$$

$E_{a}=$ Young's modulus of the adhesive

and in normalized form,

$$
\frac{\sigma_{y(\max )}}{\sigma_{a v}}=k_{2}\left(1+\frac{h}{t}\right) \sqrt{\frac{3 E_{a}\left(1-\mu^{2}\right) t}{2 k_{b} E h}}
$$

where

$$
\sigma_{a v}=\frac{T}{t}
$$

\subsubsection{Effect of Parametric Variations on Joint Strength}

In this subsection, the effect of geometric variations on the strength of the single lap joints employed in this study will be examined using Hart-Smith's computer program shown in Appendix B. The use of Hart-Smith's computer program in the elastic-plastic analysis requires the information about the dimensions of the joint and the material properties which are described in Section 4.2.4 and given in Table 4.1. The ratio of the adhesive plastic to elastic shear strains, $\gamma_{p} / \gamma_{e}$, given in Table 4.1 is determined by using HartSmith's elastic-plastic model [102]. The model is constructed in such a way that the total area under the modelled shear stress-strain curve is equal to that under the true shear 
stress-strain curve as illustrated in Figure 4.13. The shear stress-strain property of FMI300 adhesive is measured using the thick-adherend shear test [97].

There are three potential failure modes predicted by Hart-Smith's computer program, namely, adhesive failure by shear, adhesive failure by peel and adherend bending failure. The computer program produces three strength values and the lowest strength value will be the predicted failure mode. However, in the results to be shown, the main concern is to determine the relative changes of these strengths with respect to the changes in the dimension of the joint.

It is evident from Figure 4.14 that as the thickness of the adhesive layer increases, the shear strength of the joint determined from Hart-Smith's computer program will increase to a limit reaching the maximum shear strength of the adhesive. This is agreeable with the elastic analysis which shows that the stress concentrations decrease with increasing adhesive thickness as illustrated in Figure 4.7. However, it has been shown [93] that by including adhesive plasticity under shear loading in the analysis, it may decrease the stress concentrations substantially, and thus increase the estimated joint strengths, compared with a purely elastic analysis. Figure 4.15 shows the effect of elastic- plastic behaviour of the adhesive on the stress distribution in a single lap joint. As illustrated in Figure 4.9, the elastic shear stress concentrations near the ends of the overlap decrease with increasing adherend thickness. Since lower stress concentrations in the joint means that higher joint strength will be obtained, it is expected that the shear strength will increase as adherend thickness increases as depicted in Figures 4.16 and 4.17 for adhesive thicknesses of $0.076 \mathrm{~mm}$ and $0.229 \mathrm{~mm}$, respectively. Also shown in Figures 4.16 and 4.17 that the joint shear strength decreases with increasing overlap length. At first sight of the shear results shown in Figures 4.16 and 4.17, one might be led to select the minimum overlap necessary to carry the design load safely. However, if too short an overlap length is chosen high stresses will occur in the centre of the overlap upon loading and short overlap will make the joint more susceptible to creep and environmental attack [93].

One of the major concerns in designing a single lap joint is the peel stresses that exist in the joint. Designers usually attempt to reduce the peel stresses, thus allowing the 
joint to develop the full shear strength of the adhesive or, better yet, the full strength of the adherends outside the bonded joint. For joints with filamentary composite material adherends, the joint may fail by peel either in the adhesive or in the adherend, depending on whether the peel strength of the adhesive or the interlaminar strength of the composite material is greater. For the materials used in this study as specified in Table 4.1, the interlaminar or transverse tensile strength of CFRP $(=60.0 \mathrm{MPa})$ is smaller than the maximum peel strength of the adhesive $(=70.0 \mathrm{MPa})$. Thus, if the joint is to fail by peel, the failure will more likely occur in the adherend and accordingly, the lower strength value of the adherend should be entered for peel strength computations in the computer program [98]. Figure 4.18 shows that similar to the shear strength, the joint peel strength increases with increasing adhesive thickness. However, unlike the shear strength, the peel strength decreases as the adherend thickness increases and as the overlap length decreases as displayed in Figures 4.19 and 4.20 for adhesive thickness of $0.076 \mathrm{~mm}$ and $0.229 \mathrm{~mm}$, respectively. Thus, in order to minimize the peel stresses in a single lap joint, a long and thin overlap should be employed. Besides this, Hart-Smith [103] suggested the use of local tapering of the adherend at the ends of overlap to improve the peel strength of a single lap joint.

Figure 4.21 shows the change in the adherend bending strength due to variation in the adherend thickness for different overlap lengths. As can be observed in the Figure, the adherend bending strength decreases with increasing adherend thickness and with decreasing overlap length. The adherend bending strength of a joint do not vary with the thickness of the adhesive layer. The adherend bending strength of steel or CFRP lap joints used in this study is not a major concern because it is much greater than shear or peel strength of the adhesive. The adherend bending strength should be considered only for joints with long overlap length and very thin adherends. 


\subsection{Conclusions}

Factors affecting joint strength include the joint configuration, the joint dimensions and the properties of the adhesive and the adherends. Theoretical analysis can be used to study the effect of these factors on the joint strength. Elastic analysis is used to study the influence of these factors upon the stress distributions in the joint subjected to sufficiently low loads for the adhesive and the adherends to behave as elastic materials. However, most of the structural adhesives commonly used today exhibit elastic-plastic behaviour and the adhesive or the adherends of a joint may become plastic when it is subjected to high loads. Thus, the elastic-plastic analysis is required to examine the effect of geometric and/or material variations to the joint strength.

Theoretical predictions show that with all other factors kept constant, both the shear and peel strength of a single lap joint increase with increasing adhesive thickness. On the other hand, as the thickness of adherend increases, the joint shear strength increases, but the peel strength decreases. Likewise, as the overlap length of the joint increases, the shear strength decreases, but the peel strength increases. 


\section{Chapter 5}

\section{CHARACTERIZATION OF}

\section{ADHESIVELY BONDED JOINTS IN COMPOSITES}

\subsection{Introduction}

The application of adhesively bonded joints in structural components made of fibre reinforced composite materials as replacements for conventional mechanical joints has increased significantly in recent years. Mechanical fasteners such as bolts or rivets result in the cutting of fibres and the introduction of stress concentrations, both of which reduce structural efficiency. In addition, bonded joints have potential advantages in strength-to-weight ratio, design flexibility and ease of fabrication as mentioned earlier in Section 1.1. However, during fabrication of an adhesively bonded joint, physical discontinuities such as voids and unbonds can easily occur in the joint. As mentioned in Section 1.2, voids in the joint are either due to insufficient adhesive being applied or to the presence of foreign matter. Unbonds, on the other hand, are often due to the adhesive being applied to one adherend only and unevenly resulting in a gap between the adhesive and the adherend. Linbonds may also be introduced in the joint due to poor surface preparation or failure in removing completely the manufacturer's adhesive backing film. This physical discontinuity is usu- 
ally termed "zero-volume" unbond (Figure 1.1) as there is no gap between the adhesive and the adherend, but there is no bonding between them. All of these discontinuities may adversely affect the structural performance of the joint. In the event of the absence of physical discontinuities, the performance of bonded joints can also be affected by poor cohesive strength of the adhesive. Poor cohesive strength can be attributed to the effects of many factors such as improper cure, moisture, temperature, aging of the adhesive, etc. Thus, it is essential that nondestructive evaluation (NDE) methods be available to ensure that the structural performance of the bonded joint meets design and service requirements. As discussed in Chapter 2, the majority of nondestructive testing (NDT) techniques available today can readily detect physical discontinuities in the joint in terms of their location and sizes, but few techniques can provide quantitative information on the overall performance of the joint. Acousto-ultrasonic techniques, as discussed in Chapter 3, have the ability of providing quantitative information on the overall performance of adhesively bonded joints by correlating acousto- ultrasonic parameter (AUP) with the ultimate shear strength of the joint. This chapter provides the experimental results on the characterization of single lap joint composite specimens using acousto-ultrasonic as well as destructive tensile shear testing techniques. The effect of physical discontinuities such as void and unbond [81], as well as the effect of adhesive aging before bonding on the overall performance of the joint will be examined and evaluated.

\subsection{Experimental Procedure and Apparatus}

\subsubsection{Material and Test Specimens}

A composite single lap shear test specimen configuration was adopted from ASTM standard D1002-72 for the purpose of the present investigation. Six panels of $30 \times 30 \times 0.3$ $\mathrm{cm}$ were made from 24 plies of Narmco IM6/5245C graphite/epoxy unidirectional prepreg material layed up in a $\left(0^{\circ}\right)_{24}$ configuration and cured at $178^{\circ} \mathrm{C}$ and $690 \mathrm{KPa}$ per suppliers recommended cure cycle. After cure, the panels were inspected by ultrasonic C-scan to ensure that no significant defects were present. The panels were surface pretreated for 
bonding by sanding the overlap area with coarse sand papers. Two types of physical discontinuities, namely, voids and unbonds were introduced in the joint to produce variations in the joint strength.

For specimens with voids, the panels were cut in half perpendicular to the fibre direction, and bonded together with a $2.5 \mathrm{~cm}$ overlap using Ameis ican Cynamid FM300 film adhesive cured at $178^{\circ} \mathrm{C}$ and $310 \mathrm{KPa}$. Voids were created in the joint area by removing part of the adhesive in the centre of the film before bonding. After cure, the panels were inspected again in the $\mathrm{C}$-scan system and the void areas were mapped. Figures 5.1 and 5.2 show the $\mathrm{C}$-scan results for specimens with voids. The panels were then cut into test coupons $2.5 \mathrm{~cm}$ wide and $22.5 \mathrm{~cm}$ long. Five specimens were prepared with end tabs (type A) of similar thickness and material to the specimen to place the load axis in the same plane as the overlap area. The rest of the specimens were tested without end tabs (type B). The specimen types and geometries are illustrated in Figure 5.3. A total of 16 specimens was fabricated with this procedure.

For specimens with unbonds, the panels were first cut into strips of $12.5 \times 2.5 \times 0.3 \mathrm{~cm}$. Two strips were then bonded together with a $2.5 \mathrm{~cm}$ overlap using the same adhesive and curing procedures used for the case of the specimens with voids. Zero- volume unbonds (see Figure 1.1) were created in the joint by contaminating the surface of the adherend with grease in the centre of the joint area before bonding. The bonded test coupons were then inspected again using the $\mathrm{C}$-scan ultrasonic system and the unbond area was mapped as shown in Figure 5.4. A total of 10 specimens was fabricated with this procedure.

To study the effect of adhesive aging on the joint strength, the following procedure was followed. The FM300 adhesive film were aged at room temperature for periods of 3 to 8 weeks. After the aging, the composite panels were cut in half perpendicular to the fibre direction, and bonded together with a $2.5 \mathrm{~cm}$ overlap using three layers of the aged adhesive film with the cure procedures mentioned earlier. The bonded panels were then inspected again using the ultrasonic C-scan technique for any flaws. The C-scan results showed no flaws and no differences among the panels including the control panel (the panel with no adhesive aging). Five specimens were fabricated for each period of adhesive aging. 
Accordingly, a total of 35 specimens was prepared for this investigation.

\subsubsection{Acousto-Ultrasonic System}

A schematic diagram of the acousto-ultrasonic system used in this experiment is shown in Figure 5.5. An ultrasonic instrument (Automation Industries S80) was used to generate the input signals which, after passing through a repetition controller and reset timer, excited a broadband transmitting transducer (AET FC500) with $500 \mathrm{KHz}$ centre frequency. At a fixed distance away, a receiving acoustic emission transducer (AET AC375L) was used to intercept the stress waves. Both transducers were coupled to the surface using ultragel II coupling fluid. The stress waves received by the receiving transducer were amplified by $40 \mathrm{~dB}$ using a preamplifier and $125-1000 \mathrm{KHz}$ bandpass filter (AET Model 140B) and processed in real time by a standard acoustic emission instrument (AET 5000 system). The signal characteristics such as peak amplitude, ring-down count, duration and energy (Figure 3.2) were computed, displayed and stored on floppy disks. A representative waveform from the AU signal was digitized using a universal waveform analyser (Analogic Data 6000), equipped with a two-channel $36 \mathrm{MHz}$ plug-in unit (Analogic Model 630), and stored on floppy disks (Analogic 681 disk drive) for further analysis in the time and frequency domains.

To obtain reproducible AU data, the fixture shown in Figure 3.4 was used to hold the transducer on the test specimen. Hence, the transducer location and orientation as well as the pressure applied to the transducers were adjusted and maintained constant so that the received signals were repeatable during the AU measurements. For repetitive AU measurements, the transducers were first removed completely from the specimen. After cleaning the transducers and the specimen, the transducers were then coupled back to the specimen with the newly applied coupling fluid.

In the test configuration used in the present work, the transmitting transducer injects longitudinal ultrasonic waves normal to the fibres of the composite adherends in the joint section. Accordingly, taking $v=2.5 \mathrm{~km} / \mathrm{s}$ as the longitudinal velocity of graphite/epoxy laminate in the direction perpendicular to the fibres [104] and $D=6.7 \mathrm{~mm}$ as the thickness 
of the joint, resonance conditions according to Equation 3.4 should occur in the joint area at a frequency of about $373 \mathrm{KHz}$. This frequency is in the bandwidth range of both the transmitting and receiving transducers employed in this investigation, and hence optimum conditions for $\mathrm{AU}$ measurement should be achieved as explained in Section 3.3. The frequency response of the transmitting transducer (AET FC500) and the receiving transducer (AET AC375L) are illustrated in Figures 5.6 and 5.7, respectively.

\subsubsection{Mechanical Testing and Fractography}

After completion of the acousto-ultrasonic measurements, specimens were loaded in tension to failure in a universal testing machine (Instron Model 1125) using a cross-head speed of $0.5 \mathrm{~mm} / \mathrm{min}$. Wedge grips were used to hold the specimen during loading. The gripping length at each end of the specimen was approximately $2.5 \mathrm{~cm}$. The load was recorded as a function of the cross-head displacement on a chart recorder and also stored along with AU data on the AET 5000 floppy disks. After failure, the fracture surfaces were examined using an optical microscope to determine the failure modes and the void or unbond area on each specimen was measured.

\subsection{Experimental Results and Discussions}

\subsubsection{Specimens with Voids}

The typical input signal to the transmitting transducer is shown in Figure 5.8. The acoustoultrasonic waveforms in the time domain and corresponding frequency spectra for typical bonded specimens without and with void are illustrated in Figures 5.9 and 5.10, respectively. These Figures clearly show that AU signals are highly dependent on the bond condition both in terms of signal intensity and frequency content. In Figure 5.11, the normalized AU parameter (AUP/maximum AUP) is plotted against the joint shear strength (fracture load divided by the overlap area). The AUP values represent the average of 8 measurements on each specimen taken from one side of the specimen. As seen in this 
Figure, a good correlation exists between the acousto-ultrasonic parameter and the shear strength of the composite joint indicating the potential of the $\mathrm{AU}$ technique for the joint strength prediction. A correlation factor of 0.88 was obtained by fitting a straight line through the data points using the least squares method. A correlation factor of 0.83 has been reported by Dos Reis and Kautz [70] for peel strength in rubber sheet bonded to steel plate. From Figure 5.11, an estimate of strength of an unknown bonded joint can be made based on $\mathrm{AU}$ results provided that the material and geometry of the joint as well as the $\mathrm{AU}$ test conditions are exactly the same with those used to establish the correlation line.

The frequency spectra of the acousto-ultrasonic waveforms can be used to indicate the presence of voids in the joint as can be shown in Figure 5.12. When there is no voids present in the joint, the frequency spectrum of the waveform consists of one peak only occurring at the frequency of about $350 \mathrm{KHz}$, which is the resonant frequency of the receiving transducer. At the presence of voids, other peaks, occurring at lower frequencies, would start to appear in addition to the peak at the resonant frequency (see Figure 5.12).

The examination of fracture surfaces revealed that a large area of void does not necessarily correspond to a low shear strength. As shown in Figure 5.13, it is difficult to obtain a correlation between the strength of the joint and the size of void area. This is due to the fact that not only the size but also the shape and location of the void area as well as other factors may affect the strength of the joint. Wang et al [105] were also not able to find a direct relation between the void size and the fracture load of single lap-shear aluminium joints. In the specimens used in this experiment, there were basically two types of voids, namely, across the joint and at the edge of the joint. The location of the void areas in all specimens were approximately in the middle of the overlap length. Figures 5.14 to 5.16 show typical fracture surfaces of specimens without void, with voids across the joint, and at the edge of the joint, respectively. Generally, a mixed mode failure occurred consisting of cohesive failure in the adhesive, interfacial failure and fibre pull-off. At higher magnification, fine fibreglass felt in the adhesive and the presence of microvoids were observed in the regions of cohesive mode failure (Figures 5.14(b) to 5.16(b)). Fibreglass felt is commonly used for controlling the thickness of adhesive layer. The interfacial 
failure occurred primarily at the overlap ends of the joint and around the void area (Figures 5.14(c) to $5.16(\mathrm{c})$ ). The most probable site for cracks likely to grow and hence initiate failure was at the interface between the adhesive and the adherend [106]. Accordingly, it suggests that the failure of the specimens most likely initiated at the the overlap ends and around the void area where interfacial failure occurred, and the crack would then necessarily propagate into the adhesive. The interfacial failure at the overlap ends is associated with the high peel and shear stresses that occur at the ends of the overlap as shown in the stress distributions for an ideal single lap joint (Figures 4.3 to 4.12 ). The high peel stress at the overlap ends may be due to the adherend bending caused by the eccentric load path in this type of specimen. Bonding end tabs to the specimens (type A) may reduce the adherend bending and generally lead to a higher ultimate strength. However, due to factors such as the presence of void areas, end tabs in this case (type A) did not improve the ultimate strength to any noticeable degree. Table 5.1 summarizes the fractographic results and gives the failure modes of each specimen along with the type and size of the void area as well as the shear strength.

\subsubsection{Specimens with Unbonds}

Figures 5.17 and 5.18 show acousto-ultrasonic waveforms in the time domain for typical bonded joint specimens without and with unbond, respectively. As shown in the Figures, the AU signal levels between these two types of specimens do not vary as considerably as those between the specimens without and with void as shown in Figures 5.9 and 5.10, respectively. This is expected because those specimens with voids contain greater volume of air which would attenuate acousto-ultrasonic waves than those without voids, resulting in reduced AU signals received at the receiving sensor. Figure 5.19 shows the correlation between the normalized AUP and the joint shear strength (fracture load/overlap area) with a correlation factor of 0.94 obtained using the least squares method. The AUP data was normalized for the effect of variation in adherend thickness (range from 3.02 to 3.53

$\mathrm{mm}$ ) and with respect to the maximum AUP for the given data in order to obtain a relative measure of AUP. In this regard, the AUP data measured on the joint area were divided 
by the ALP value measured on the adherend of the specimen away from the orerlap area and then by the maximum value of $A L^{-} P$ for the given data. The value of normalized $A T^{-} P$ in the Figure represents an average of 4 measurements on each specimen. As illustrated in Figure 5.20, the ALP ralue measured on the adherend of the specimen reduces as the adherend thickness increases. This could be due to the change in the path of the ware propagation in the composite adherends and also to the change in the ratio of fibre to resin volume which resulted from the fabrication process.

Similar to the specimens with roids. the frequency spectra of the acousto-ultrasonic wareforms show rariation due to the presence of unbonds in the joints (Figure 5.21). In the absence of unbonds (well bonded). the frequency spectrum of the waveform shows a major peak occurring at about the resonant frequency of the receiving transducer (approximately: $360 \mathrm{KHz}$ ), but as the unbond size increases. there are additional peaks appearing at higher frequencies than the resonant frequency of the receiving transducer.

The fact that the unbond size is not the only factor that affects the joint strength can be illustrated in Figure 5.22, which shows no correlation between the unbond size and the joint strength. As explained in Section 5.3.1, the joint strength depends on many other factors such as the shape and location of the unbonds, and also the stiffness of the composite adherends which may vary due to variation in the ratio of fibre to resin volume. Examination of the fracture surfaces revealed that there was generally a mixed mode of failure consisting of cohesive failure in the adhesive, interfacial failure at the free edges between the adhesive and the adherend, fibre pull-off, and interlaminar failure in the first few plies of the composite adherend near the adhesive (see Figure 5.23). It was observed that for joints with predominantly interlaminar failure mode. the joint strength generally tends to be lower. Table 5.2 provide the summary of the mechanical testing and fractographic results as well as the unbond size and the thickness of the adherend.

\subsubsection{The Effect of Adhesive Aging}

Since variation in adhesive thickness may affect the $\mathrm{AL}^{-}$measurement and the joint strength (see Section 4.3.2), it is important to determine the adhesive thickness of each specimen be- 
Sore attempting to eraluate the performance of the joint specimens. The achesite titimess הas measured with an optical microscope and by using the u'trasonic C-scan pu'se-echo method (see Section 2.2.1). Lsing the transducer frequency of $10 \mathrm{MHz}$ and the signa: processing unit (DAIA 6000 in Figure 5.5). it was possible to separate the echoes ai the interfaces of the joint specimen. Figure 5.24 illustrates a typical ultrasonic puise-echo signal showing the front and back echoes of the top and bottom of the adherends. B: using the acoustic relocity in the epoxy as $2.4 \mathrm{~km} / \mathrm{s}, 10 \mathrm{i}_{\mathrm{j}}$ and taking half of the time delay difference between echoes 2 and 3 shown in Figure 5.24. the adhesire thickness of the joint can be determined. Both measurements of adhesire thickness by ultrasonic pulse-echo technique and optical microscope show good agreements as can be seen in Figure 5.25.

The $A T^{-}$results were affected by the rariation in adhesive thickness as shown in Figures 5.26(a) and (b) for two representative groups of specimens. As can be seen in these Figures. the $A L^{-} \mathrm{P}$ which is supposed to be constant for a giren group of specimens increases linearly with increasing adhesive thickness for each group of the specimens. Accordingiv. the AL'P values were normalized with respect to the rariation in adhesire thickness. In this regard. the ALP ralue obtained for a given specimen was divided by the adhesive thickness of the specimen, and then by the maximum $A \tau^{-} \mathrm{P}$ for the given data in order to obtain a relative measure of the ALP. Hence. the normalized $A L^{-} P$ can be expressed as :

$$
\text { Normalized AUP }=\frac{\text { AUP }_{\text {nor- }}}{\text { AUP }_{\text {norm(Trz- })}}
$$

where

$$
\begin{aligned}
\text { AUP }_{\text {notm }} & =\frac{\text { AUP measured for a given specimen }}{\text { Adhesive thickness of the specimen }} \\
\text { AUP }_{\text {norm(max) }} & =\text { Maximum value of the quantity for the given data }
\end{aligned}
$$

The change in normalized ALP (Equation 5.1) with increasing time of adhesive aging is shown in Figure 5.27. The Figure shows the scatter of fire data points for each group and the arerage value of the data points. As indicated in Figure $5.2 \bar{\tau}$. the acousto- ultrasonic parameter of the specimens generally shows a reduction with increasing aging time.

The effect of adhesive thickness on the joint strength can be analysed by using the elastic-plastic analysis described in Section 4.3 and the computer program shown in Ap- 
pendix B. Using this computer program, the joint failure mode predicted in this investigation is likely to be by peel and the effect of adhesive thickness on the joint peel strength was obtained as shown in Figure 4.18. Thus, from the data shown in Figure 4.18, it is possible to determine the relative change in the joint strength due to variation in adhesive thickness. The joint strength measured from the destructive test was then normalized with respect to this relative change in the joint strength to eliminate the effect of variation in adhesive thickness. Accordingly, the normalized joint strength for each specimen can be expressed as :

$$
\text { Normalized joint strength }=\frac{\tau_{\text {norm }}}{\tau_{\text {norm }(\max )}}
$$

where

$$
\tau_{\text {norm }}=\frac{A}{R}
$$

$A$ is the actual joint strength obtained from destructive test for a specimen with a given adhesive thickness; $R$ is the joint strength determined from the computer program in Appendix $\mathbf{B}$ for the given adhesive thickness; and $\tau_{n o r m(\max )}$ is the maximum value of the quantity in the given data.

Figure 5.28 shows the change in the normalized joint strength expressed in Equation 5.2 as a function of aging time of the adhesive film. The scatter and the average value of the five data points of each group is shown in the Figure. As the aging time of the adhesive increases, the joint strength is expected to decrease because for an extended period of aging, the adhesive film becomes more polymerized and does not convert to the fluid during the curing process and therefore bond formation will not be effective [33]. However, as illustrated in Figure 5.28, the joint strength shows a reduction of only about $15 \%$ for the aging time of 8 weeks in room temperature. This relatively small reduction in strength with aging time could be due to the fact that the adhesive used in this study was a hightemperature curing adhesive. At room temperature, which is below the glass transition temperature of the adhesive, slow cure of the adhesive would take place [108].

Comparison between Figures 5.27 and 5.28 indicates that both the acousto-ultrasonic evaluation and the destructive testing results of the specimens with aged adhesive exhibit a similar trend with aging time. Figure 5.29 shows a plot between the normalized AUP 
and the normalized joint strength. From the plot, a correlation factor of $0 . \$ 3$ was obtanes? by using the least squares method.

Fractographic examination of the fracture specimens shows that the failure modes were predominantly cohesive failure in the adhesive and interlaminar failure in the composite adherend with some fibre pull-off and interfacial failure. Figure 5.30 illustrates the different modes of failure observed for the joint specimens used in this study: There was no significant trend of the failure mode that could be related to the adhesive aging time. but it was observed that specimens with longer period of adhesive aging tend to have relatively more interfacial failure.

\subsection{Conclusions}

The shear strength of single lap joint composite specimens with introduced roids or unbonds has been eraluated using the acousto-ultrasonic and destructive shear testing techniques. Good correlations were found between the acousto-ultrasonic parameter (ALP) and the shear strength of the joint specimens. This indicates that the shear strength of a single lap joint can be estimated nondestructively using acousto-ultrasonic technique prorided that the measurement system is calibrated for material and geometrical variations. Acousto-ultrasonic technique can also be used to indicate the presence of roids and unbonds in a joint by examining the frequency spectra of the acousto- ultrasonic waveform. Poor correlations were found between the joint shear strength and the size of roid or unbond.

The effect of adhesive aging on the strength of the composite joint specimens has also been evaluated using the acousto-ultrasonic nondestructive and destructive shear testing techniques. The adhesive thickness of the joint specimens were determined by using ultrasonic pulse-echo and optical microscope methods. Both methods showed good agreements in the measurement of adhesive thickness. Since rariation in the adhesive thickness affected both the ALP and the joint strength. they were normalized with respect to the variation. It was found that the normalized $A L P$ correlated well with the normalized joint 
strength. The correlation suggests that the strength of a joint with aged adhesive can be nondestructively assessed in a quantitative manner using the acousto-ultrasonic technique.

The failure modes of adhesively bonded joints in composites generally consisted of cohesive failure in the adhesive, interfacial failure, fibre pull-off and interlaminar failure. The interfacial failure mode generally occurred at the free edges of the joint specimens and around the void areas. It was observed that the joint specimens with more interfacial failure tend to have lower joint strength. 


\section{Chapter 6}

\section{EVALUATION OF STEEL BONDED JOINTS AT ELEVATED TEMPERATURES}

\subsection{Introduction}

One of the limitations in using adhesively bonded joints in structural components, as explained in Chapter 1, is that the mechanical performance of the joints during its service may be adversely affected by exposure to the environmental conditions such as moisture and high temperature. It has been found that the strength of adhesively bonded joints deteriorates rapidly during exposure to high temperature environments [109]. It has also been shown that bonded joints do not regain their initial strength after they have been exposed to elevated temperatures especially when the temperature approaches the glass transition temperature of the adhesives [57]. The degradation in strength is greater when the joints are exposed repeatedly to elevated temperatures. As a result, structural components with adhesively bonded joints are often restricted to applications where temperatures remain within a range that is tolerable to the adhesive. However, such components may inadvertently be exposed to elevated temperatures in situations such as mechanical overheating or fire. Thus, it is important that nondestructive evaluation (NDE) methods be available 
to ensure that the structural performance of the bonded joints is not compromised by environmental effects orer the service life of the structure.

As discussed in previous chapters, most of the $\mathrm{NDE}$ techniques currently available are sensitive to physical defects in materials but few techniques can provide information on those intrinsic material properties that affect structural performance. In the case of evaluating bonded joints subjected to elevated temperatures, there is no physical discontinuities to be detected. Thus, most NDE techniques usually used as detectors of physical discontinuities are ineffective in this case. Acousto- ultrasonic techniques, on the other hand. can provide quantitative information on those intrinsic material properties that affect the mechanical performance of the joint, hence will be found useful in this study. It has been demonstrated that the acousto-ultrasonic techniques can be used in the nondestructive evaluation of the shear strength of adhesively bonded joints in aluminium [68] and the shear strength of the adhesive bond between rubber and steel plates [70]. In the previous chapter. good correlations were found between the acousto-ultrasonic parameter and the shear strength of single lap joint composite specimens containing voids or unbonds.

In this chapter, the experimental results obtained using acousto-ultrasonic as well as destructive tensile shear testing techniques in the characterization of single lap steel specimens are presented. Thermal degradations of the bonded specimens were achieved by subjecting the specimens to progressively increasing temperatures or to cyclic thermal exposure [110]. Acousto- ultrasonic evaluation of the specimens was carried out by analysing the acousto-ultrasonic signals in time and frequency domains. An analysis of wave propagation through the adherend- adhesive system, which supports the experimental data, is also discussed.

\subsection{Experimental Procedure and Apparatus}

\subsubsection{Material and Test Specimens}

The single lap shear test specimen configuration was adopted from ASTM standard D1002$i 2$ for the purpose of the present investigation. A plain carbon (AISI-S.AE 1020) steel 
strip $25.4 \mathrm{~mm}$ wide and $3.0 \mathrm{~mm}$ thick was cut into test coupons of $127.0 \mathrm{~mm}$ in length. The steel test coupons were then ground and sanded to remove sharp edges and to ensure that surfaces were flat. The steel surfaces were then sandblasted to remove inactive oxide and hydroxide layers and to obtain an increased effective surface area for adhesion. After sandblasting, the steel bars were degreased by immersing them in chloroethane solvent for about 30 minutes and brushing the surfaces with the solvent. As soon as the steel bars were removed from the solvent, they were bonded together with a $25.4 \mathrm{~mm}$ overlap. American Cynamid FM300 modified epoxy adhesive film with a cure process of 3 hours at $177^{\circ} \mathrm{C}$ and $600 \mathrm{KPa}$ was used for bonding. The adhesive film has a service temperature range of $-55^{\circ} \mathrm{C}$ to $150^{\circ} \mathrm{C}$ [111]. An adhesive thickness of $0.05 \mathrm{~mm}$ in the joint area was obtained by separating the two adherends with aluminium shims of that thickness during the cure process.

A total of 24 specimens were prepared for the present investigation. To evaluate the effect of temperature variation on the AUP and joint strength, three specimens were used for measuring the change in AUP with increasing temperature and 15 specimens were loaded in tension to failure at 5 different temperatures $\left(30,60,90,120\right.$, and $\left.150^{\circ} \mathrm{C}\right)$ with three specimens tested at each temperature. In order to evaluate the effect of cyclic thermal exposure on the AUP and joint strength, three specimens were exposed repeatedly to temperature of $150^{\circ} \mathrm{C}$ for about 1 hour and then cooled down to the room temperature. These three specimens were evaluated by comparing with three as-fabricated specimens using acousto- ultrasonics and destructive shear testing.

\subsubsection{Acousto-Ultrasonic Measurement Apparatus}

A schematic diagram of the acousto-ultrasonic system arrangement used to evaluate steel specimens at room and elevated temperatures is shown in Figure 6.1. An ultrasonic instrument (Automation Industries S80) was used to generate the input signals which, after passing through a repetition controller and reset timer, excited a broadband transmitting transducer (AET FC500, frequency range of 0.1 to $2 \mathrm{MHz}$ with $0.5 \mathrm{MHz}$ nominal centre frequency). At a fixed distance away, a receiving acoustic emission transducer (AET 
AC1000, $1 \mathrm{MHz}$ nominal centre frequency) was used to intercept the stress wares. The stress waves received by the receiving transducer were amplified by $40 \mathrm{~dB}$ using a preamplifier (AET Model $140 \mathrm{~B}$, equipped with a 0.125 to $2 \mathrm{MHz}$ bandpass filter) and processed in real time by a standard acoustic emission instrument (AET 5000 system). The signal characteristics such as peak amplitude, ring-down count, duration and energy were computed, displayed and then stored on floppy disks. A representative waveform from the AU signal was digitized using a universal waveform analyser (Analogic DATA 6000) equipped with a two-channel, $36 \mathrm{MHz}$ plug-in unit (Analogic Model 630) and stored on floppy disks (Analogic 681 disk drive) for further analysis in the time and frequency domains. The room-temperature AU measurements were carried out using a spring-loaded fixture shown in Figure 6.1(a) which maintained a constant pressure and a fixed distance between the centres of the transducers for improved reproducibility. In this way, the $A U$ signals obtained from one specimen could be evaluated with respect to other specimens. The transducers were coupled to the surface of the specimen using Ultragel II coupling fluid. A total of four measurements were taken on each specimen.

The AU measurements at high temperatures were carried out using a different transducer-specimen arrangement as illustrated in Figure 6.1(b). In this case, an ultrasonic probe (Gamma Aerotech, $2.25 \mathrm{MHz}$ nominal centre frequency) was used as the transmitting transducer and a resonant acoustic emission sensor (AET AC1000, $1 \mathrm{MHz}$ nominal centre frequency) was used as a receiving transducer. The transducers were coupled to the specimen with constant force clamps that provided sufficient pressures for transducerspecimen coupling without the need for coupling fluid which was ineffective at elevated temperatures. Heat was applied locally to the joint area using a hot plate, but due to heat conduction, the adherends and the transducers also experienced somewhat elevated temperatures. The temperature difference between one side of the specimen and the other side in the joint region was less than $1^{\circ} \mathrm{C}$. AU measurements were taken on each specimen while heated from $30^{\circ} \mathrm{C}$ to $150^{\circ} \mathrm{C}$ at $10^{\circ} \mathrm{C}$ intervals and the data were normalized with respect to the results obtained at $30^{\circ} \mathrm{C}$.

For the configuration used in the present tests (Figure 6.1), longitudinal ultrasonic 
waves were injected by the transmitting transducer into the joint specimen. Accordingly, taking $v=5.90 \mathrm{~km} / \mathrm{s}$ as the longitudinal velocity of steel [107] and $D=6.0 \mathrm{~mm}$ as the thickness of the joint. resonance conditions should occur in the joint area at frequencies of about $0.98 \mathrm{MHz}$ for $n=1$ and $1.96 \mathrm{MHz}$ for $n=2$ (Equation 3.4). These frequencies are in the bandwidth range of the transmitting and receiving transducers utilized in the present investigation.

\subsubsection{Mechanical Testing and Fractography}

After completion of the acousto-ultrasonic measurements, specimens were loaded in tension to failure in a universal testing machine (Instron Model 1125, equipped with a model 3111 environmental chamber). Wedge grips were used to hold the specimen during loading. The specimen was placed inside the chamber and heated up to the desired temperature which ranged from $30^{\circ} \mathrm{C}$ to $150^{\circ} \mathrm{C}$ in $30^{\circ} \mathrm{C}$ intervals. The load was applied at a cross-head speed of $0.2 \mathrm{~mm} / \mathrm{min}$ after the test temperature had been maintained for at least 10 minutes. After failure, the fracture surfaces were examined using an optical microscope to determine the failure modes.

\subsection{Experimental Results}

\subsubsection{Effect of Change in Temperature}

The change in normalized AUP with increasing temperature of the adhesively bonded specimens is shown in Figure 6.2. The value of normalized AUP in the Figure represents the average of measurements on three specimens and the range of scatter in the data is indicated by vertical lines. The AUP data shown in Figure 6.2 had been compensated for the effect of temperature variations on the adherends and the transducers. The compensation was accomplished by dividing the AUP data obtained for adhesively bonded joint specimens by those measured under the same test conditions for a specimen consisting of only the adherend material. As indicated in Figure 6.2, the acousto- ultrasonic response 
of the bonded steel specimens generally decreases with increasing temperature.

The change in the acousto-ultrasonic response with temperature can also be seen by analysing the $\mathrm{AU}$ signals in the frequency domain as illustrated in Figure 6.3 . At $30^{\circ} \mathrm{C}$, there are basically four major peaks in the frequency spectrum occurring at about 0.7 , $0.85,1.10$ and $1.20 \mathrm{MHz}$. As the temperature increases, the amplitudes of the peaks at about $0.7,0.85$ and $1.10 \mathrm{MHz}$ start to decrease, while the peak at about $1.20 \mathrm{MHz}$ increases in amplitude relative to other peaks in the frequency spectrum and shifts slightly toward the region of $1.15 \mathrm{MHz}$. The peak at about $1.20 \mathrm{MHz}$ is believed to result from the adherend material alone as can be seen from the frequency spectra of $\mathrm{AU}$ signals for the specimen which had no adhesive joint (Figure 6.4). The frequency spectra of Figure 6.4 consist of a peak at about $1.19 \mathrm{MHz}$ increasing in amplitude and shifting slightly toward $1.17 \mathrm{MHz}$ as temperature rises. Also, as indicated in Figure 6.3, there is a peak at about $1.25 \mathrm{MHz}$ which starts to appear at higher temperatures. The origin of this peak is not fully understood yet.

Figure 6.5 shows the change in average shear strength (the breaking load divided by the overlap area) of the joint specimens as a function of test temperature. Each data point in Figure 6.5 represents the average of three specimens and the range of scatter in the data is shown by vertical lines. Since the whole specimen with the grips was placed inside the environmental testing equipment and the desired test temperature was maintained for at least 10 minutes before loading, it can be assumed that there was no temperature variation within the specimen. However, temperature fluctuations within the environmental chamber were of the order of $\pm 3^{\circ} \mathrm{C}$. The results shown in Figure 6.5 for single lap joint specimens using FM300 modified epoxy adhesive film generally exhibit a reduction in strength of the joint as temperature increases which agrees well with the results previously presented by Kausen [112] for a similar type of adhesive.

Examination of the fracture surfaces of the specimens revealed that a mixed mode failure consisting of interfacial and cohesive failures had taken place. Interfacial failure was indicated when adhesive remained attached to one of the adherends while leaving the other adherend surface clean. Cohesive failure was indicated when adhesive remained on both 
adherend surfaces. As illustrated in Figure 6.6. the failure mode from room temperature to $90^{\circ} \mathrm{C}$ consisted mainly of cohesive failure with an insignificant amount of interfacial failure. In this temperature range, the shear strength of adhesive bond decreases slightly with the change of test temperature. However, at temperatures of $120^{\circ} \mathrm{C}$ and above, interfacial failure was the predominant mode. In fact, the failure mechanism of specimens tested at $150^{\circ} \mathrm{C}$ was almost completely by the interfacial mode. In this temperat ure range, a sharp decline in shear strength takes place which is probably associated with the interfacial failure mode. This trend can be observed in Figure 6.5.

By comparing Figures 6.2 and 6.5, it can be seen that both the acousto-ultrasonic and destructive testing of adhesively bonded specimens show similar trends with temperature. The correlation is illustrated in Figure 6.7 where the normalized AUP is plotted against the normalized shear strength (shear strength divided by maximum shear strength). A correlation factor of 0.964 is obtained by fitting a straight line through the data points using the least squares method.

\subsubsection{Effect of Cyclic Thermal Exposure}

Table 6.1 shows the acousto-ultrasonic and shear strength results for the bonded specimens repeatedly heated to about $150^{\circ} \mathrm{C}$ for one hour and cooled down to the room temperature as compared with the as-fabricated specimens. All the $\mathrm{AU}$ and mechanical tests in this case were carried out at room temperature. It can be seen from Table 6.1 that the specimens subjected to cyclic thermal exposure generally have lower AUP and average shear strength values than those of the as-fabricated specimens. The normalized AUP values in the table represent the average of four $\mathrm{AU}$ measurements on each specimen.

The correlation between the acousto-ultrasonic parameter and the shear strength of the specimens subjected to cyclic thermal degradation is shown in Figure 6.8. The correlation factor in this case is 0.955 . Fracture surface examination by optical microscopy showed that some parts of the adhesive in the specimens which were subjected to cyclic thermal exposure had been thermally degraded (burned). Those specimens showed more evidence of interfacial failure than did the as-fabricated specimens (Figure 6.9). 


\subsection{Discussions}

According to Rokhlin $[31,32]$, the change in elastic modulus of the modified epoxy adhesive (such as the one used in present study) with temperature is due to the known thermomechanical behaviour of polymers which, at high temperatures, behave as a low-modulus, rubber-type material while, at low temperatures, show the behaviour of a high-modulus, glass-type material. Since the cohesive strength of a given material correlates with its elastic modulus, a change in the elastic modulus of the material is normally associated with a corresponding change in strength [31,113]. Hence, as the elastic modulus of a polymeric adhesive decreases with increasing temperature, it is expected that the cohesive stren.y... of the adhesive layer will also decrease.

Likewise, the elastic modulus affects the velocity of sound waves propagating through a material as expressed in the following equation [107]:

$$
v=K_{1} \sqrt{\frac{E}{\rho}}
$$

where $v$ is the acoustic velocity, $E$ is the elastic modulus, $\rho$ is the density of the material and $K_{1}$ is a function of Poisson's ratio. The relationship of acoustic velocity with temperature for epoxy resin has been studied before [114] and it has been found that the acoustic ve of epoxy resin below its glass transition temperature exhibits a monotonic decrease with increasing temperature. The product of the density of a material and the acoustic velocity of sound is a parameter known as the acoustic impedance $(Z)$, i.e.:

$$
Z=v \rho
$$

Substituting Equation 6.1 into 6.2,

$$
Z=K_{1} \sqrt{E_{\rho}}
$$

The density of the adhesive layer in a bonded joint is also expected to decrease slightly at higher temperatures due to thermal expansion and gasification effects. Since both the velocity of sound waves and the density of adhesives decrease with increasing temperature, the acoustic impedance of adhesives should also decrease as temperature rises. Acoustic 
impedance controls the acoustic energy transmission through a layered medium such as an adhesively bonded joint. The acoustic energy transmission coefficient, $D_{t}$, for plane waves at normal angles of incidence travelling from one material with an acoustic impedance of $Z_{1}$ to another material with an acoustic impedance $Z_{2}$ is given by [107]:

$$
D_{t}=\frac{2 Z_{2}}{Z_{1}+Z_{2}}
$$

For the configuration used in the present study (Figure 6.1), the sound waves travel from the first steel adherend $\left(Z_{1}=45 \times 10^{6} \mathrm{~kg} / \mathrm{m}^{2} \mathrm{~s}\right)$ to the adhesive $\left(Z_{2} \approx 2.7 \times 10^{6} \mathrm{~kg} / \mathrm{m}^{2} \mathrm{~s}\right)$ and then to the second steel adherend. Since $Z_{1} \gg Z_{2}$, the acoustic transmission at the steel-adhesive interface can be approximated by:

$$
D_{t} \approx \frac{2 Z_{2}}{Z_{1}}
$$

The acoustic transmission coefficient at the second interface which is adhesive to steel can be written as

$$
D_{t}=\frac{2 Z_{1}}{Z_{1}+Z_{2}}
$$

which for $Z_{1} \gg Z_{2}$,

$$
D_{t} \approx \frac{2 Z_{1}}{Z_{1}}=2
$$

With test temperatures up to only $150^{\circ} \mathrm{C}$, the effect of temperature on the steel adherends will be negligible. Thus, the acoustic impedance of the adherends, $Z_{1}$, can be assumed to be constant with temperature in this test. From Equation 6.5, the coefficient of transmission and hence, the stress wave transmission efficiency, is proportional to the acoustic impedance of the adhesive and is expected to decrease as the temperature increases. Since the AUP is a measure of the efficiency of stress wave transmission, it is therefore expected that the AUP value will decrease as temperature increases. Vary [6] has also reported a correlation between stress wave propagation and tensile strength of graphite/epoxy composites due to the dependence of both $\mathrm{AU}$ stress wave propagation and strength on the elasticity (stiffness) of the materials. 


\subsection{Conclusions}

The effect of temperature on the properties of adhesively bonded steel specimens has been evaluated using acousto- ultrasonics and destructive shear testing. A good correlation has been found between the acousto-ultrasonic parameter and the shear strength of single lap joint steel specimens subjected to increasingly high temperatures. Both AUP and strength showed a decline with increasing temperature. It is possible that this decline is associated with the temperature dependence of the elastic modulus of the adhesive layer. A good correlation was also found between the acousto-ultrasonic parameter and the shear strength of the specimens subjected to cyclic thermal degradation. These correlations suggest that acousto-ultrasonic techniques can be used to nondestructively monitor or assess the strength degradation of adhesively bonded joints during or after exposure to elevated temperatures.

Examination of the fracture surfaces by optical microscopy showed that the failure mode in adhesively bonded single lap joint steel specimens generally consisted of cohesive and interfacial failures, but as the test temperature increased, the interfacial failure was found to be increasingly predominant. More interfacial failure was also noticed in the specimens subjected to cyclic thermal degradation than the as-fabricated specimens. 


\section{Chapter 7}

\section{EVALUATION OF FOAM CORE ALUMINIUM PANELS}

\subsection{Introduction}

Recently, investigations on the possible application of foam core aluminium bonded sandwich panels in transport vehicles have been carried out. Such panels have advantages over the commonly used sheet metals in terms of strength to weight ratio, stiffness, heat insulation and noise absorption. However, foam core aluminium panels are susceptible to impact and fatigue which could induce internal damages. In some cases, these damages are not visible at the aluminium surface but have a significant effect on the load bearing performance of the structure. In addition, the foam core aluminium panels consist of two substrates of totally different materials and thus proper surface preparations of the substrates are critically important to ensure proper adhesion between the adhesive and the two substrates. As a result, there is a requirement for nondestructive testing techniques to detect the existence of fatigue or impact damaged areas economically and reliably as well as to detect poor surface preparation in the foam core aluminium panels.

Nondestructive evaluation of the panels using conventional ultrasonic techniques are usually difficult due to the highly attenuative foam core and thin aluminium sheets. Acousto- ultrasonic techniques, on the other hand, have been reported to have the ca- 
pability to detect inadequate bond areas between a metal foil honeycomb and porous (highly attenuative) wireform substrate [79]. It has also been reported that the acoustoultrasonic technique can be used to detect impact damaged areas in graphite/epoxy panels [12]. Based on these studies, it is believed that acousto-ultrasonic techniques can be used to detect damaged areas and poor surface preparation in the foam core aluminium panels.

This chapter provides experimental results obtained by using acousto-ultrasonic technique to inspect and evaluate foam core aluminium panels [115]. Acousto-ultrasonic inspection of the panels will be focussed on detection of void, teflon insert as well as fatigue and impact damaged areas in the panels. Comparisons with other nondestructive testing techniques in terms of detecting the flaws and damaged areas will be made. Evaluation of the panels with different foam surface preparation techniques by acousto-ultrasonic and destructive peel testing techniques will also be presented.

\subsection{Experimental Procedure and Apparatus}

\subsubsection{Test Panels}

The panels, which were fabricated and supplied by the Kingston Research and Development Centre (KRDC) of Alcan International Limited, consisted of a $38 \mathrm{~mm}$ thick foam core sandwiched between two $1 \mathrm{~mm}$ thick aluminium sheets. Aluminium sheets were bonded adhesively to the foam core using proprietary adhesives and bonding procedures. Figure 7.1 shows a foam core aluminium test specimen and its dimensions.

Four types of defects were introduced to the test specimens as listed in Table 7.1 and described in the following:

(a) A void area approximately $25 \mathrm{~mm}$ in diameter was deliberately introduced at the interface between the foam core and aluminium during fabrication.

(b) An artificial disbond using a $20 \mathrm{~mm}$ diameter disk of teflon tape was inserted between the foam core and aluminium face during fabrication. 
(c) Fatigue damage was induced by cyclic loading of an as-fabricated specimen in threepoint bending.

(d) Impact damage was introduced in two specimens with a drop weight tower at impact energies in the range of 1.4 to 14 joules using either a $12.5 \mathrm{~mm}$ diameter semispherical head or a nearly flat $(22.5 \mathrm{~cm}$ diameter $)$ indentor. Figure 7.2 shows a photograph of the panels after impact. Each panel contained impact damage which was either visible or barely visible at the surface. Table 7.1 provides the impact energy and visual description of the impact sites. A more detailed description of the apparatus, procedures, energy levels and visual observations associated with the impact tests is given in Reference [116].

To study the effect of surface preparation on the peel strength of the panels, four different methods of foam surface preparations were carried out before bonding as listed in the following :

(a) As-received (AR)

(b) Old roller (OR)

(c) New roller (NR)

(d) New roller with two layers of adhesive (NR2)

The rollers for foam surface preparation consist of pins which are to make holes in the foam core so that there will be an increase in the bonding area. The old and new rollers (OR and NR) differ in terms of the size and the spacing of the pins. For each method mentioned above, the surfaces of the substrates were either prepared with or without priming, thus resulting in 8 different foam surface preparations.

\subsubsection{Acousto-Ultrasonic Test Procedure}

The acousto-ultrasonic measurements to detect flaws and damaged areas in the foam core aluminium panels were carried out using an Acoustic Emission Technology Corporation 
AET-206AU instrument. The instrument was equipped with a hand-held probe fixture for the purpose of scanning the panels. The fixture housed the transmitting and receiving transducers (both had a nominal centre frequency of $350 \mathrm{KHz}$ ) which were dry-coupled to the test specimen by two rotating wheels as illustrated in Figure 7.3. The instrument is capable of measuring the Stress Wave Factor (SWF) and the root mean square (RMS) amplitude of the received signals described in Section 3.2. The latter was used in the present investigation for two reasons. First, the measurement of RMS amplitude takes into account the amplitudes of each oscillations of a waveform as in the case of AUP measurement (section 3.2). Secondly, it was found to produce more repeatable data for the panels in this investigation.

The spacing between the transmitting and receiving wheels was adjusted to $2.5 \mathrm{~cm}$. The $\mathrm{AU}$ inspection was performed by simply rolling the probe manually over the test specimen in the longitudinal direction and taking the RMS readings every $2.5 \mathrm{~cm}$. The reading was assigned to the point on the panel in the middle of the contact points of the transmitting and receiving wheels. A total of 14 readings were taken in the longitudinal direction in each linear scan. For the next scan, the position of the transducer was translated by $1.25 \mathrm{~cm}$ across the specimen and the $\mathrm{AU}$ measurement was repeated as before until all of the surface of the panel had been scanned. A total of five scans were performed on each panel and the average values of the data with respect to each point were used. The AU inspection data were then plotted as a function of location of the probe on the panel.

For evaluating the performance of the panels with different foam surface preparation, the acousto-ultrasonic test set-up was the same as that shown in Figure 5.5. However, in the present experiment, two AET AC1000 transducers with $1 \mathrm{MHz}$ nominal centre frequency were used as transmitting and receiving transducers. The spacing between the centres of the transducers was $10.0 \mathrm{~cm}$. An averaging process of the $\mathrm{AU}$ waveforms was carried out during the acousto-ultrasonic data acquisition in order to minimize noise. In the averaging process, the transmitting transducer injected 20 pulses successively into the specimen and the data acquisition system (DATA 6000 in Figure 5.5) performed a linear average of the 20 received waveforms to produce an averaged waveform. Since noise normally created was 
random signal in the waveforms, they would be reduced or cancelled out in the arcringing process. The AUP measurement process of the acousto- ultrasonic data was repeated $=1 \times$ times.

\subsubsection{Mechanical Testing and Fractography}

After completion of the nondestructive testing of the foam core aluminium panels, destructive testing of the panels was undertaken for flaw verification. For this purpose, internal flaws were opened to the surface by drilling a fine hole in the aluminium face and a liquid dye penetrant was used to stain the flaw surfaces. Then, the aluminium faces were peeled off to permit visual examination of the damaged areas.

The destructive test for evaluating the panels which were fabricated with different surface preparation techniques involves climbing drum peel tests for foam cored structures following the ASTM standard D1781. Testing of the panels was performed on the Instron 1125 load frame using the low capacity $(100 \mathrm{~kg}$ ) load cell. Procedure used for testing was as outlined in ASTM standard D1781. The peeled surfaces of the aluminium sheet and the foam core were then inspected to determine the failure modes. The destructive tests of the panels were carried out by KRDC of Alcan International Limited.

\subsection{Experimental Results and Discussions}

Figures 7.4, 7.5 and 7.6 illustrate three-dimensional plots of AU measurements on panel Fo/Al-2, 3 and 4, respectively. The plots shown in the Figures represent the relative RAIS amplitude variations measured along the panels. The base plane shown in the plots corresponds to well bonded areas in the panels while increases in RMS amplitude relative to the base plane correspond to fatigue or impact damaged areas. An increase in RMS amplitude when the AU transducers are located on top of the damaged areas can be explained in the following manner: Longitudinal waves from the transmitting transducer propagate into the aluminium face sheet of the panel, but by the process of mode conversion, plate waves are generated that propagate along the aluminium face sheet [36]. If good bond exint 
between the aluminium sheet and the foam core, the plate waves are partially transmitted through the bond into the attenuative foam. However, fatigue and impact damaged areas prevent the plates wave from travelling into the foam, thus resulting in an $\mathrm{AU}$ signal level increase received by the receiving transducer.

The plots in Figures 7.4 to 7.6 also provide RMS amplitude information which has a good correlation with the severity of damage. The extent of impact damage depends on the impact energy and the size of the impact head indentor. Both panels Fo/Al-3 and Fo/Al4 were impact tested at approximately the same impact energies but with two different impact head sizes. Panel Fo/Al-3 which was impact tested with a smaller impact head was more severely damaged (deeper dents) than panel Fo/Al-4 for a given impact energy. This agrees well with the AU measurements illustrated in Figures 7.5 and 7.6 showing that at a given impact energy the peaks for panel Fo/Al-3 are higher than those for panel Fo/Al-4. It is also evident from these Figures that for each panel, the peaks of the RMS amplitude correlate well with the impact energies.

Using the AU techniques, the teflon insert and the void in the adhesive could also be detected although the sensitivity was not as good as it was for fatigue and impact damage. The same foam core aluminium panels have also been evaluted with other nondestructive testing techniques such as the ultrasonic c-scan and the mechanical impedance analysis (MIA) [115]. Due to the highly attenuative foam core, the ultrasonic c-scan throughtransmission method was ineffective because it was not possible to propagate ultrasound through the panels. Difficulty was also encountered when the ultrasonic c-scan pulse-echo method was used to evaluate the panels because of the thin face sheet of aluminium. With this method, it was possible to detect the circular void area, but not the areas of fatigue and impact damage. The use of mechanical impedance analysis, on the other hand, made it possible to detect both the teflon insert as well as the fatigue and impact damage. But it was not possible to detect the $25 \mathrm{~mm}$ diameter void area in panel Fo/Al-1 (Table 7.1). Work has been carried out on the application of thermography to the detection of missing adhesive (void) at the aluminium foam boundary and positive results have been obtained [63]. 
Figures 7.7, 7.8 and 7.9 show the acousto-ultrasonic waveforms for panels with surface preparation techniques: as- received (AR), as-received with priming (ARP) and new roller with priming (NRP), respectively. By applying primer on the bonded components before bonding improved the adhesion. Consequently, it resulted in a decrease in the AU signal level as can be compared in Figures 7.7 and 7.8. Surface preparation with NRP (Figure 7.9) resulted in an even reduced AU signal intensity. The reason for this which has been explained earlier is that proper surface preparation which produces better adhesion allows part of the acousto-ultrasonic stress waves to be transmitted through the bond into the attenuative foam core. The normalized acousto- ultrasonic parameter (AUP divided by the maximum AUP) was plotted against the peel torque of the panels as shown in Figure 7.10. The peel torque is the average torque required to bend and peel the aluminium sheet from the foam core. The method of calculating the peel torque is outlined in ASTM D1781. A correlation factor of 0.93 was obtained by best fitting a straight line through the data points using the least square method. The value of the normalized AUP data in the Figure represents the average of six measurements and the value of the peel torque is obtained from the average results of six specimens.

Figures 7.11 to 7.18 display the representative fracture surfaces of the panels with different surface preparation techniques. The types of failure modes for the panels consisted of interfacial failure between the adhesive and the foam core, cohesive failure in the adhesive and in the foam core. It can be seen from these Figures that the amount of cohesive failure in the adhesive relates to the peel torque obtained. The failure mode of the as-received panel was completely interfacial failure as can be seen from its "smooth" surface of the foam core (Figure 7.11), and this corresponds to the lowest peel torque. On the other hand, the panel with NRP surface preparation (Figure 7.18) which has the highest peel torque exhibits a predominant cohesive failure in the adhesive. Table 7.2 provides a summary of the destructive test results as well as the AUP data. 


\subsection{Conclusions}

The acousto-ultrasonic technique using RIS amplitude of signals can be used to detect the teflon insert. lack of adhesive. fatigue and impact damage areas in the foam core aluminium panels. The technique provided three-dimensional plots of RMS amplitude as a function of location of the transducer on the surface of the panels. A good correlation was obtained between the RMS amplitude of the AL signals and the sererity of impact damage which in turn depends on the impact energy and the impact head size. The use of conrentional ultrasonic techniques to detect flaws and damaged areas was ineffective. but the use of mechanical impedance analysis (MIA) and thermography has been successful.

The effect of different foam surface preparation techniques on the performance of the foam core aluminium panels has been evaluated using the acousto-ultrasonic and destructive peel testing techniques. A good correlation was found between the acousto-ultrasonic parameter ( $A L^{-} P$ ) and the peel torque of the panels. The failure mode of the panels generally consisted of interfacial failure. cohesive failure in the adhesive and in the foam. 


\section{Chapter 8}

\section{EVALUATION OF BONDED JOINTS UNDER TENSILE LOADING}

\subsection{Introduction}

Acousto-ultrasonic technique has been shown in previous chapters to be capable of providing nondestructive evaluation of material property variations in a quantitative manner. The technique has proven particularly useful for quantifying relative joint strength by correlating the acousto-ultrasonic parameter with the joint strength. The acousto-ultrasonic approach can then be used to estimate the joint strength based on the correlation, after the measurement system has been calibrated against the variations in material and geometry of the test specimen. Vary and Bowles [117] showed the use of this approach to estimate the interlaminar shear strength of graphite/polyimide composite laminates. In this case, the strength estimations of the test specimens were carried out before the specimens were loaded to failure.

Strength estimation of a test specimen can also be made by acousto-ultrasonic monitoring of the specimen during loading as in the case of acoustic emission ( $\mathrm{AE}$ ) technique. When a material is subjected to tensile loading, it experiences microstructural changes 
and these changes affect the acousto-ultrasonic stress wave propagation characteristics. Hemann and Baaklini [118] observed the change in attenuation of stress waves as the tensile stress for a graphite/epoxy composite laminate was increased. Williams et al. [73] also noticed the change in the AU measurement of nylon ropes as the tension of the ropes was increased. It is believed that the change in AU stress wave propagation due to the microstructural changes in a stressed material will provide useful information in estimating the fracture strength of the material. In this chapter, the acousto- ultrasonic approach is used to monitor the change in stress wave propagation characteristics in adhesively bonded joint specimens in composites and steel under tensile loading to fracture. The purpose of the present chapter is to determine if there is a correlation between the change in acoustoultrasonic parameter and the change in tensile load applied to the joints. An approach based on the AU waveform partitioning method $[8,9]$ was used to study the components of $\mathrm{AU}$ waveforms for certain frequency ranges.

\subsection{Experimental Procedure and Apparatus}

\subsubsection{Material and Test Specimens}

There were two types of single lap joint test specimens to be used in this investigation, namely, adhesively bonded joint specimens in graphite/epoxy composites and steel. The composite specimens were fabricated in a similar fashion with those specified in Section 5.2.1, while the procedure of fabricating the steel specimens was outlined in Section 6.2.1. However, surface contamination with fingerprints on the adherend surface after surface treatment was used to obtain variations in the joint strength for both types of the specimens. A total of seven specimens was fabricated for composite specimens and five for steel specimens. 


\subsubsection{Acousto-Ultrasonic System Arrangement}

A block diagram of the acousto-ultrasonic system arrangement used in the present investigation for monitoring a specimen under loading is shown in Figure 8.1. and the apparatus for measuring the acousto-ultrasonic parameter during loading is depicted in Figure 8.2. All equipments used for AU measurements were similar to those specified in Section 5.2.2. However, the transmitting and receiving transducers used were either AET AC1000 (centre frequency of $1 \mathrm{MHz}$ ) and $\mathrm{AET} \mathrm{AC} 375$ (centre frequency of $0.375 \mathrm{MHz}$ ). respectively for composites specimens or Gamma Aerotech (2.25 $\mathrm{MHz}$ centre frequency) and AET AC1000 (1 MHz centre frequency), respectively for steel specimens. The spacing between the centres of the transmitting and receiving transducers were $152 \mathrm{~mm}$. Both transducers were coupled to the surface of the specimen with Ultragel II coupling fluid using constant force clamps shown in Figure 8.3.

\subsubsection{Test Procedure}

After coupling both transmitting and receiving transducers to the test specimens, the specimens were mounted on a universal testing machine (Instron Model 1125) and were loaded in tension using a cross-head speed of $0.2 \mathrm{~mm} / \mathrm{min}$. Wedge grips were used to hold the specimen during loading. The grip area at each end of the specimen was approximately $2.5 \mathrm{~cm}$ in length. During loading of the test specimens, acoustic emissions were inevitably generated due to internal response (see Section 2.4). In order to reduce the effect of these emissions on the acousto-ultrasonic waveforms, two approaches were used.

For a composite specimen, the load was increased from zero to fracture with every load interval of $1.225 \mathrm{kN}$, twenty high amplitude input pulses were injected successively in a very short time into the specimen. The $\mathrm{AU}$ waveforms received were then linearly averaged to give an averaged waveform with reductions in noise and acoustic emission, assuming that noise and acoustic emissions generated were random signals and were small compared to the AU signals. In order to have low level of acoustic emission signals relative to the $\mathrm{AU}$ signals, the gain of the measurement system was reduced considerably whereas the 
amplitude of the input pulse was increased.

For a steel specimen, the load was increased from zero to $1.225 \mathrm{kN}$ and held constant. During the constant load, when it was observed from AET 5000 (Figure 5.5) that there were no more internal generation of acoustic emissions from the specimen, an input pulse was injected into the specimen. The resultant $\mathrm{AU}$ waveform was then captured and stored in the floppy disks. The load was again increased by $1.225 \mathrm{kN}$ and the same procedure was repeated until it reached fracture load.

\subsubsection{Signal Processing Procedure}

Even with the procedures to reduce the effect of acoustic emissions on the AU signals described in the previous subsection, it was observed that as load was increased, especially when it was approaching the fracture load, a significant amount of acoustic emissions internally generated from the specimen superimposed with the AU signals. To reduce further the effect of acoustic emission on the $\mathrm{AU}$ results, signal filtering of the $\mathrm{AU}$ waveforms from certain frequency ranges is required. The signal filtering is achieved by using an approach based on the AU waveform partitioning approach described by Kautz $[8,9]$, in which the frequency spectrum of the waveform is divided by several frequency ranges and the waveform is analyzed with respect to these ranges. To obtain an AU waveform consisting a certain range of frequency content, the following procedure was carried out. First, the magnitude and phase of the original full frequency domain signals were zeroed for frequencies outside the range of interest. After the zeroing process, the frequency domain signals were then transformed back to produce filtered time domain signals using the inverse Fast Fourier Transform (FFT) algorithm [119]. 


\subsection{Experimental Results and Discussions}

\subsubsection{Composite Specimens}

It was observed that the frequency spectra of the acoustic emissions internally generated from adhesively bonded composite specimens generally consisted of frequencies below $550 \mathrm{kHz}$. Figure 8.4 illustrates the frequency spectra of some typical acoustic emissions from a composite specimen when the load was $60 \%, 75 \%, 80 \%$ and $95 \%$ of the fracture load. Accordingly, the frequency range of interest should be above $550 \mathrm{kHz}$ in order to reduce the effect of acoustic emission on the AU signals. For an analysis of the AU waveform with respect to different frequency ranges, the frequency spectrum of the waveform was divided into 4 frequency ranges : $0-1.70 \mathrm{MHz}$ (full spectrum), $0-0.275 \mathrm{MHz}, 0.275-0.550$ $\mathrm{MHz}$ and $0.550-0.875 \mathrm{MHz}$. Figure 8.5 shows typical $\mathrm{AU}$ waveforms which have been "digitally filtered" according to these frequency ranges for a representative specimen (For signal processing procedure, please refer to Section 8.2.4). The normalized AUP of these waveforms were plotted against the normalized load as shown in Figure 8.6. The AUP values were normalized relative to the initial AUP value obtained before loading while the loads were normalized with respect to the fracture load. As demonstrated in Figure 8.6, a good correlation exists between the normalized AUP and the normalized load for the frequency range of 0.550 to $0.875 \mathrm{MHz}$, whereas irregular trends were observed for other frequency ranges. It can also be seen in the Figure that during low load ( 0.0 to 0.3 of fracture load), the AUP values for all frequency ranges exhibit similar trend, suggesting that when the load was low, insignificant amount of acoustic emissions were generated that would affect the AU signals. The characteristic of the normalized AUP vs the normalized load for the frequency range of 0 to $0.275 \mathrm{MHz}$ is similar to that for the full frequency range ( 0 to $1.70 \mathrm{MHz}$ ). The similarity indicates that the dominant components of the $\mathrm{AU}$ waveforms appeared in the frequency range of 0 to $0.275 \mathrm{MHz}$. By virtue of the fact that a good correlation exists for the frequency range of 0.550 to $0.875 \mathrm{MHz}$, the normalized AUP of the waveform with this range of frequency content was plotted against the normalized load for all specimens as shown in Figure 8.7. The fracture load of each specimen are given 
in Table 8.1. By using the least squares method, a straight line was fitted through the data points in Figure 8.7 which can be expressed by :

$$
\text { AUP }_{n}=0.9872-0.4916 P_{n}
$$

or,

$$
P_{n}=2.0081-2.0342 \mathrm{AUP}_{n}
$$

where

$$
\begin{aligned}
\text { AUP }_{n} & =\text { normalized AUP } \\
& =\frac{\text { AUP value measured at a load }}{\text { AUP value measured before loading }} \\
P_{n} & =\text { normalized load } \\
& =\frac{\text { Load at which the AUP value is measured }}{\text { Fracture load }}
\end{aligned}
$$

Thus, an estimation of failure load of an unknown composite specimen can be made by using Equation 8.2, provided that the material and geometry of the specimen as well as all test conditions are the same with those used to establish Equation 8.2. In the estimation, it is necessary to measure the AUP values before loading and at any load, preferably at a load before permanent damage is done on the specimen.

\subsubsection{Steel Specimens}

Frequency spectrum analyses of the $\mathrm{AU}$ waveforms for all steel specimens reveal that there are three prominent peaks occurring around $0.3,0.6$ and $1.1 \mathrm{MHz}$ as can be shown in Figure 8.8, which illustrates a typical $\mathrm{AU}$ waveform in time and frequency domains for a representative specimen. Using the approach based on the AU waveform partitioning method $[8,9]$, the frequency spectra of the $A U$ waveforms were divided into four frequency ranges, i.e., 0.0 to $1.7 \mathrm{MHz}$ (full spectrum), 0.0 to $0.4 \mathrm{MHz}, 0.4$ to $0.8 \mathrm{MHz}$ and 0.8 to $1.2 \mathrm{MHz}$ in order to study each component of the $\mathrm{AU}$ waveform comprising of these frequency ranges. Figure 8.9 shows typical $\mathrm{AU}$ waveforms which contain these frequency ranges for a representative specimen. The change in normalized AUP (AUP values / the 
ALP value before loading) with increasing normalized load (load / the fracture load) for a typical steel specimen is given in Figure 8.10. The normalized ALP for the frequency range of 0.4 to $0.8 \mathrm{MHz}$ decreases proportionally with increasing load. while for other frequency ranges, the normalized AUP values decrease during the initial part of the loading and then increase when the load approaches the fracture load. As shown in Figure 8.10, the dominant components of the $\mathrm{AU}$ waveform in this case occur in the frequency range of $0 . \mathrm{S}$ to $1.2 \mathrm{MHz}$.

Figure 8.11 shows the normalized AUP for the frequency range of 0.4 to $0.8 \mathrm{MHz}$ plotted against the normalized load for all the specimens with their fracture loads listed in Table 8.1. Similar to the composite joint specimens, a straight line can also be fitted through the data points of the steel joint specimens using the least squares method. The line can be expressed by :

$$
\text { AUP }_{n}=0.9864-0.2662 P_{n}
$$

or,

$$
P_{n}=3.7055-3.7566 \text { AUP }_{n}
$$

where $A U P_{n}$ and $P_{n}$ are defined in Equations 8.3 and 8.4, respectively. As indicted in Figure 8.11 or Equation 8.6, the AUP value reduced to about $70 \%$ of its initial value (the AUP value before loading the specimen) as the load was increased until catastrophic joint failure occurred (when the normalized load is equal to one). Thus, by using the correlation line between the AUP and the load expressed in Equation 8.6. an estimation of the fracture load of an unknown steel joint specimen is possible.

\subsection{Conclusions}

Acousto-ultrasonic (AU) technique was used to monitor an adhesively bonded joint specimen in steel or composites under tensile loading. The main problem in this investigation was that acoustic emissions (AE) were inevitably generated from the specimen during the loading and the $A E$ signals superimposed with the $A U$ signals. In order to reduce the 
effect of acoustic emissions on the AU signals, specific test procedures for AU measurements were carried out. The effect of acoustic emissions was further reduced by utilizing an approach based on the $\mathrm{AU}$ waveform partitioning method $[8,9]$. In this approach, the frequency spectra of the $\mathrm{AU}$ waveforms were divided into several frequency ranges and the AU waveforms consisting of these frequency ranges were studied. The AUP of the waveform with a certain range of frequency content in which insignificant amount of acoustic emissions occurred from the specimens was used to correlate with the tensile load. A good correlation was found between the change in AUP and the change in load for this frequency range. Based on these correlation lines, an estimation of the breaking load of an unknown joint specimen can be made, provided that the material and geometry of the specimen as well as all test conditions are the same with those used to establish the correlation line. 


\section{Chapter 9}

\section{CONCLUDING REMARKS AND RECOMMENDATIONS}

An adhesively bonded joint can offer considerable advantages over a conventional mechanical joint, particularly in terms of ease of fabrication and weight saving, and its use has increased significantly. However, the primary disadvantage of the bonded joint is the inability to insure that a finished joint will actually meet the strength and service requirements for which it was designed. There is therefore an increasing need for more effective methods of nondestructive testing (NDT). Most NDT methods developed for evaluating the bonded joint can readily detect flaws, but it is often difficult to correlate a detected flaw to the overall performance of the joint. Acousto-ultrasonic technique, which combines beneficial aspects of ultrasonics and acoustic emission, can provide quantitative evaluation on the overall performance of the bonded joint.

Acousto-ultrasonic (AU) technique has been used to evaluate the shear strength variations of single lap bonded joints in graphite/epoxy composites due to voids, unbonds and adhesive aging. It has also been used to measure the strength degradation of steel bonded joints subjected to elevated temperatures. Foam core bonded aluminium sandwich panels which can possibly be used in transport vehicles have been inspected with the $\mathrm{AU}$ technique to detect voids, fatigue and impact damaged areas, as well as to detect poor surface preparation of the panels. Quantitative evaluation on the overall performance 
of the bonded joints or the bonded panels involves correlating the joint strength with the acousto-ultrasonic parameter (AUP), a parameter measurable from the AU waveform. Good correlations have been found between the AUP and the shear strength of the bonded joints in composites and steel, as well as between the AUP and the peel strength of the foam core aluminium panels. These correlations suggest that an estimation of the joint strength can be made provided that the $\mathrm{AU}$ measurement system is calibrated for variations in the material and geometry of the specimen. The acousto-ultrasonic technique has also been used to monitor an adhesively bonded joint specimen under tensile loading. The correlations found between the change in AUP and the change in tensile load can be used to estimate the joint strength.

The results of the acousto-ultrasonic measurement system can be affected by many factors including the pressure and the coupling fluid applied to the transducer and the specimen. These factors affect the reproducibility of the AU measurements. Attempts to eliminate the coupling fluid which sometimes can be impractical in the AU measurements have been carried out by using dry coupling method or contactless laser probes $[120,121]$. However, these coupling methods still require more research on their application to acoustoultrasonic technique so that reproducibility of measurement is improved. Other alternative methods of coupling can also be used and have been discussed by Vary [80].

Variations in the material and the geometry of test specimens affect the results of the AU measurement and the destructive testing differently. The effect of these variations on AU stress wave propagation characteristics should be studied and understood. To do this, it is useful to attempt to trace wave paths, to track multiple reflections, and to gauge depth of penetration of the stress waves. These variations on the destructive testing results should be calibrated by conducting a series of mechanical testing with the aids of theoretical analyses on the stress distribution in the bonded joints.

Acousto-ultrasonic waveform is a very complex waveform, resulting from multiple reflections of the different modes of wave propagation. With the advanced technology of signal processing available today, a large number of acousto-ultrasonic signal parameters can be extracted from the waveform. These parameters are affected by a number of mechanical 
properties and morphological conditions of the bonded joint. This suggests that pattern recognition method is required to analyse all of these acousto-ultrasonic signal parameters in order to determine the state of the bonded joint. The method involves statisticallybased data generation, feature extraction, and classification. By using samples containing known states (i.e.. training samples) significant discriminatory pattern can be defined and used for feature extraction. Then, the most likely states in an unknown bonded joint can be identified and associated with particular acousto-ultrasonic signal parameters. 


\section{REFERENCES}

1. Shields, J., 'Adhesive Bonding', Oxford University Press, London, 1974, p.1.

2. Eley, D.D., and Tabor, D., 'Fundamentals of Adhesive Joints', Adhesion, Eley, D.D., Ed., Oxford University Press, London, 1961, p.1.

3. Shields, J., 'Adhesive Handbook', 3rd. Ed., Butterworths, London, 1984, pp.3-4.

4. Vary, A., 'Acousto-Ultrasonic Characterization of Fiber Reinforced Composites', Materials Evaluation, Vol.40, No.6, May 1982, pp.650-662.

5. Vary, A. and Bowles, K.J., 'An Ultrasonic-Acoustic Technique for Nondestructive Evaluation of Fiber Composite Quality', Polymer Engineering and Science, Vol.19, No.5, April 1979, pp.373-376.

6. Vary, A. and Lark, R.F., 'Correlation of Fiber Composite Tensile Strength with the Ultrasonic Stress Wave Factor', Journal of Testing and Evaluation, Vol.7, No.4, July 1979, pp.185-191.

7. Henneke II, E.G., et al, 'A Study of the Stress Wave Factor Technique for the Characterization of Composite Materials', NASA Contractor Report 3670, February 1983.

8. Kautz, H.E., 'Ultrasonic Evaluation of Mechanical Properties of Thick, Multilayered, Filament Wound Composites', NASA Technical Memorandum 87088, September 1985.

9. Kautz, H.E., 'Acousto-Ultrasonic Verification of the Strength of Filament Wound Composite Material', NASA Technical Memorandum 88827, July 1986.

10. Williams, J.H.Jr. and Lampert, N.R., 'Ultrasonic Evaluation of Impact-Damaged Graphite Fiber Composite', Material Evaluation, Vol.38, No.12, December 1980, pp.68-72.

11. Duke, J.C.Jr., et al, 'Ultrasonic Stress Wave Characterization of Composite Materials', NASA Contractor Report 3976, May 1986. 
12. Green, J.E. and Rodgers, J., 'Acousto-Ultrasonic Evaluation of Impact-Damaged Graphite Epoxy Composites', 27th National SAMPE Symposium, May 4- 61982 , pp.428-439.

13. Dos Reis, H.L.M. and McFarland, D.M.,'On the Acousto-Ultrasonic Non-destructive Evaluation of Wire Rope Using the Stress Wave Factor Technique', British Journal of Nondestructive Testing, Vol.28, No.3, May 1986, pp.155-156.

14. Schliekelmann, R.J., 'Non-destructive Testing of Adhesive Bonded Joints', AGARD Lecture Series No.102, March 1979, pp.8.1-8.37.

15. Schliekelmann, R.J., 'Non-destructive Testing of Adhesive Bonded Metal-to-Metal Joints-1', Non- destructive Testing, Vol.5, No.2, April 1972, pp.79-86.

16. Kim, D.H. and Stutliff, E.F., 'The Contact Potential Difference (CPD) Measurement Method for Prebond Nondestructive Surface Inspection', SAMPE Quarterly, Vol.9, No.4, July 1978, pp.59-63.

17. Hagemaier, D.J., 'Bonded Joints and Non- destructive Testing-Bonded Honeycomb Structures- 1', Non-destructive Testing, Vol.4, No.6, December 1971, pp.401-406.

18. Rose, J.L. and Meyer, P.A., 'Ultrasonic Procedure for Predicting Adhesive Bond Strength', Materials Evaluation, Vol.31, No.6, June 1973, pp.109-114.

19. Hagemaier, D. and Fassbender, R., 'Nondestructive Testing of Adhesive Bonded Structure', SAMPE Quarterly, Vol.9, No.4, pp.36-58.

20. Botsco, R.J., 'High Resolution Ultrasonics', Materials Evaluation, Vol.25, No.4, April 1967, pp.76-82.

21. Passerell, N.D. and Foster, A.C., ' Ultrasonic Inspection of Large Solid Fuel Booster Nozzle', Materials Evaluation, Vol.25, No.3, March 1967, pp.53-57.

22. Segal, E. and Rose, J.L., 'Nondestructive Testing Techniques for Adhesive Bond Joints', Research Techniques in Nondestructive Testing, Vol.4, Sharpe, R.S., Ed., Academic Press, New York, 1980, pp.275-316.

23. Curtis, J., 'Non-destructive Testing of Adhesively Bonded Structures with Acoustic Methods', Ultrasonic Testing, Szilard, J., Ed., John Wiley \& Sons Ltd., New York, 1982 , pp.495-554. 
24. Guyott, C.C.H., Cawley, P. and Adams, R.D., 'The Non-destructive Testing of Adhesively Bonded Structure : A Review', Journal of Adhesion, Vol.20, No.2, 1986, pp.129-159.

25. Alers, G.A., Flynn, P.L. and Buckley, H.J., 'Ultrasonic Technique for Measuring the Strength of Adhesive Bonds', Materials Evaluation, Vol.35, No.4, April 1977, pp.7784.

26. Rose, J.L. and Meyer, P.A., 'Ultrasonic Determination of Strength', Journal of Adhesion, Vol.8, No.2, 1976, pp.150-153.

27. Kline, R.A., Hsiao, C.P. and Fidaali, M.A., 'Nondestructive Evaluation of Adhesively Bonded Joints', Journal of Engineering Materials and Technology, Vol.108, No.3, July 1986, pp.214-217.

28. Djordjevic, B.B. and Venables, J.D., 'Nondestructive Evaluation of Bonded Metal and Composite Structures', Proceedings of 13th Symposium on Nondestructive Evaluation, Leonard, B.E., Ed., San Antonio, Texas, April 1981, pp.68- 76.

29. Fahr, A. and Charlesworth, A.M., 'An Introduction to the Ultrasonic C-scan Inspection of Advanced Composite Materials', NRC, NAE LTR-ST-1602, November 1986.

30. Thompson, D.O., Thompson, R.B. and Alers, G.A., 'Nondestructive Measurement of Adhesive Bond Strength in Honeycomb Panels', Materials Evaluation, Vol.32, No.4, April 1974, pp.81-85,92.

31. Rokhlin, S.I., 'Adhesive Joint Characterization by Ultrasonic Surface and Interface Waves', Adhesive Joint : Their Formation, Characteristics and Testing, Mittal, K.L., Ed., Plenum Press, New York, 1984, pp.307-345.

32. Rokhlin, S.I., 'Evaluation of the Curing of Structural Adhesives by Ultrasonic Interface Waves. Correlation with Strength,' Journal of Composite Materials, Vol.17, No.1, January 1983, pp.15-25.

33. Rokhlin, S.I., 'Adhesive Joint Evaluation by Ultrasonic Interface and Lamb Waves', Materials Analysis by Ultrasonics-Metals, Ceramics, Composites, Vary, A., Ed., Noyes Data Co., New Jersey, 1987, pp.290-301.

34. Kline, R.A. and Hashemi, D., 'Ultrasonic Guided- Wave Monitoring of Fatigue Damage Development in Bonded Joints', Materials Evaluation, Vol.45, No.9, September 1987, pp.1076-1082. 
35. Chapman, G.B.II, 'A Nondestructive Method of Evaluating Adhesive Bond Strength in Fiberglass Reinforced Plastic Assemblies', Joining of Composite Materials, ASTMI STP 749, Kedward, K.T., Ed., American Society for Testing and Materials, 1981, pp.32-60.

36. Pike, R.A. and Williams, R.S., 'Ultrasonic Assessment of Cure Rate Effects in Bonded Honeycomb Structures', Adhesive Joints : Their Formation, Characteristics and Testing, Mittal, K.L., Ed., Plenum Press, New York, 1974, pp.369- 380.

37. Schliekelmann, R.J., 'Nondestructive Testing of Adhesively Bonded Joints', Adhesion : Fundamentals and Practice, Gordon and Breach Science Publishers Inc., New York, 1969, pp.246- 261.

38. Schliekelmann, R.J., 'Nondestructive Testing of Adhesively Bonded Metal-to-Metal Joints-2', Non- destructive Testing, Vol.5, No.3, June 1972, pp.144-153.

39. Clemens, R.E., 'Evaluation of the Fokker Bond Tester System for Nondestructive Testing FM-47 Adhesive Bonded Honeycomb and Metal-to-Metal Structure', Paper Presented at an American Society for Nondestructive Testing Technical Meeting, California, February 1962.

40. Brown, A.F., 'Materials Testing by Ultrasonic Spectroscopy', Ultrasonics, Vol.11, No.5, September 1973, pp.202-210.

41. Brown, A.F., 'Ultrasonic Spectroscopy', Ultrasonic Testing, Szilard, J., Ed., John Wiley \& Sons Ltd., New York, 1982, pp.167-215.

42. Llyod, E.A., 'Non-destructive Testing of Bonded Joints-A Case of Testing Laminated Structures by Wide-Band Ultrasound', Nondestructive Testing- Research and Practice, Vol.7, No.6, December 1974, pp.331-334.

43. Llyod, E.A. and Brown, A.F., 'Recent Developments Non-destructive Testing of Adhesive-Bonded Joints', Adhesion 2, Allen, K.W., Ed., Applied Science Publishers Ltd., London, 1978, pp.133-144.

44. Llyod, E.A. and Wadhawani, D.S., 'Ultrasonic Spectroscopy and the Detection of $\mathrm{Hy}$ drothermal Degradation in Adhesive Bonds', Adhesion 4, Allen, K.W., Ed., Applied Science Publishers Ltd., London, 1980, pp.159-174.

45. Chang, F.H., Flynn, P.L., Gordon, D.E., and Bell, J.R., 'Principles and Application of Ultrasonic Spectroscopy in NDE of Adhesive Bonds', IEEE Transactions on Sonics and Ultrasonics, Vol.SU-23, No.5, September 1976, pp.334-338. 
46. Adams, R.D. and Cawley, P., 'Vibration Techniques in Nondestructive Testing', Research Techniques in Nondestructive Testing, Vol.8, Sharpe, R.S., Ed., Academic Press, New York, 1985, pp.303-360.

47. Cawley, P., 'The Inspection Method Of Non- destructive Inspection', NDT International, Vol.17, No.2, April 1984, pp.59-65.

48. Cawley, P., 'The Operation of NDT Instruments Based on the Impedance Method', Composite Structures, Vol.3, No.3\&4, 1985, pp.215-228.

49. Cawley, P., 'The Sensitivity of the Mechanical Impedance Method of Nondestructive Testing', NDT International, Vol.20, No.4, August 1987, pp.209- 215.

50. Hagemaier, D.J., 'Bonded Joints and Non- destructive Testing-Bonded Honeycomb Structures- 2', Non-destructive Testing, Vol.5, No.1, February 1972, pp.28-48.

51. Botsco, R., 'The Eddy-Sonic Test Methods', Materials Evaluation, Vol.26, No.2, February 1968, pp.21-26.

52. Schmitz, G. and Frank, L., 'NDT Evaluation of the Strength of Bonded Materials', NASA Contractor Report CR-67983, September 1966.

53. Beal, J., 'Ultrasonic Emission Detector Evaluation of Strength of Bonded Materials', NASA Technical Memorandum NASA TMX-53602, October 1966.

54. Pollock, A.A., 'Stress-Wave Emission In N.D.T.', Non-destructive Testing, Vol.2, No.3, August 1969, pp.178-182.

55. Curtis, G.J., 'Acoustic Emission Energy Relates to Bond Strength', Non-destructive Testing, Vol.8, No.5, October 1975, pp.249-257.

56. Speake, J.H. and Curtis, G.J., 'Ultrasonic and Stress-Wave-Emission Non-destructive Testing of Polymeric Adhesives', Plastics and Rubber : Materials and Applications, Vol.1, No.3/4, September 1976, pp.193-204.

57. Hill, R., 'The Use of Acoustic Emission for Characterizing Adhesive Joint Failure', NDT International, Vol.10, No.2, April 1977, pp.63-72.

58. Brockmann, W. and Fischer, T., 'Acoustic Emission Analysis as a Method of Testing Adhesive Metal Joints', 22nd National SAMPE Symposium and Exhibition, San Diego, California, April 26-28, 1977, Diversity-Technology Explosion, Vol.22, pp.277300 . 
59. Treca, M.. XDI of Bonded Structures'. Mon- destructive Inspection Practices. Bolis. E., Ed.. AGARD-AG-201-Tol.II. October 1975. pp.529-5i i.

60. Segal. E.. Thomas. G.. and Rose. J.. 'Hope for Solving the Adhesive Bond Nightmare?". Proceedings of the 12th Symposium on Nondestructive Evaluation. San Antonio, Texas. April 24-26, 1979, pp.269-281.

61. Pye. C.J. and Adams. R.D.. 'Detection of Damage in Fibre Reinforced Plastics Lsing Thermal Fields Generated During Resonant Tibration', XDT International. Vol.40. No.3. June 1981. pp.111- 118.

62. Reynolds. W... and Wells, G.M.. 'Tideo-Compatible Thermography'. British Journal of Non-destructive Testing. Vol.26. To.1. January 1984. pp.40-44.

63. Maldague. X.. Cielo, P.. Ashley, P.J. and Farahbahhsh, B.. Thermographic NDT of Aluminium Laminates'. C.S.N.D.T. Journal. Vol.8. No.4. July/August 1987. pp.4450.

64. Kersch. L.A., 'Laminate Structure Inspection'. Holographic Nondestructive Testing. Erf, R.K.. Ed.. Academic Press. New York, 1974. pp.303-322.

65. Crane. R.L.. 'A New Penetrant for Composite and Adhesively Bonded Structures'. Materials Evaluation. Vol.35, No.2. February 1977. pp.54-55.

66. Segal, Y.. Notea, A. and Segal, E., 'A Systematic Evaluation of Nondestructive Testing Methods', Research Techniques in Nondestructive Testing, Sharpe. R.S.. Ed.. Vol.3. Academic Press, New York, 1977. pp.293-321.

67. Williams, J.H.Jr., Lee, S.S. and Wang, T.K., 'Nondestructive Evaluation of Strength and Separation Modes in Adhesively Bonded Automotive Glass Fibre Composite Single Lap Joints', Journal of Composite Materials, Vol.21. No.1, January 198T. pp.14-35.

68. Kapoor, A. and Prakash, R., 'Strength of Adhesive Bonded Joints'. International Conference on Advances in Fracture Research (Fracture 84). Proceedings of the 6th. New Delhi. India, 1984, pp.2649-2655.

69. Dos Reis, H.L.M., Bergman, L.A. and Bucksbee. J.H.. Adhesive Bond Strength Quality Assurance Using the Acousto-Ultrasonic Technique'. British Journal of Aondestructive Testing, Vol.28, No.6, November 1986. pp.35i-358. 
70. Dos Reis. H.L.MI and Kautz, H.E., Nondestructive Evaluation of Adhesive Bond Strength Using the Stress Wave Factor Technique'. Journal of Acoustic Emission. Vol.5, No.4, October-December 1986, pp.144-147.

71. Nayeb-Hasemi, N., Cohen, M.D., Zotos, J. and Poormand, R., 'Ultrasonic Characteristics of Graphite/Epoxy Composite Material Subjected to Fatigue and Impacts'. Journal of Nondestructive Evaluation, Vol.5, No.3/4, December 1986. pp.119- 131.

72. Srivastava, V.K. and Prakash, R., 'Fatigue Life Prediction of Glass Fibre-Reinforced Plastics Using the Acousto-Ultrasonic Technique', International Journal of Fatigue. Vol. 9, No. 3, July 1987, pp.175-178.

73. Williams, J.H.Jr., Hainsworth, J. and Lee, S.S., 'Acoustic-Ultrasonic Nondestructive Evaluation of Double-Braided Nylon Ropes Using the Stress Wave Factor', Fibre Science and Technology, Vol.21, No.3, 1984, pp.169-180.

74. Dos Reis, H.L.M. and McFarland, D.M.,'On the Acousto-Ultrasonic Characterization of Wood Fiber Hardboard', Journal of Acoustic Emission, Vol.5, No.2, April-June 1986, pp.67-70.

75. Phani, K.K., Niyogi, S.K., Maitra, A.K. and Roychaudhury, M., Strength and Elastic Modulus of a Porous Brittle Solid : An Acousto-Ultrasonic Study', Journal of Material Science, Vol.21, No.12, December 1986, pp.4335-4341.

76. De, A., Phani, K.K. and Kumar, S., 'Acousto- Ultrasonic Study on Glass-Ceramics in the System $\mathrm{MgO}-\mathrm{Al}_{2} \mathrm{O}_{3}-\mathrm{SiO}_{2}$ ', Journal of Materials Science Letters, Vol.6, No.1, pp.17-19.

77. Phani, K.K. and Bose, N.R., 'Hydrothermal Aging of CSM-Laminate During Water Immersion An Acousto- Ultrasonic Study', Journal of Materials Science, Vol.21, No.10, October 1986, pp.3633-3637.

78. Phani, K.K. and Bose, N.R., 'Hydrothermal Aging of Jute-Glass Fibre Hybrid Composites -An Acousto- Ultrasonic Study', Journal of Materials Science, Vol.22, No.6. June 1987, pp.1929-1933.

79. Vary, A., Moorhead, P.E. and Hull, D.R., 'Metal Honeycomb to Porous Wireform Substrate Diffusion Bond Evaluation', Spring ASNT Meeting, Boston, Massachusetts, April 1982, pp. 185-191. 
80. Vary, A., 'The Acousto-Ultrasonic Approach', NASA Technical Memorandum $\$ 98+3$. April 1987.

81. Fahr, A., Lee, S. and Tanary, S., 'Application of Acousto-Liltrasonic Technique to the Characterization of Adhesively-Bonded Joints in Fibre Reinforced Composites', LTR-ST-1625, National Research Council Canada, May 1987.

82. Matthews, F.L., Kilty, P.F., and Godwin, E.W., 'A Review of the Strength of Joints in Fibre- Reinforced Plastics : Part 2. Adhesively Bonded Joints', Composites, Vol. 13, No.1, January 1982, pp. 29-37.

83. Volkersen, O., 'Die Nietkraftverteilung in Zugbeanspruchten Nietverbindungen mit Konstanten Laschenquerschnitten', Luftfahrtforschung, Vol. 15, 1938, pp. 41-47.

84. Goland, M. and Reissner, E., 'The Stresses in Cemented Joints', Journal of Applied Mechanics, Vol. 11, No. 1, March 1944, pp. A-14 A-27.

85. Lubkin, J.L. and Reissner, E., 'Stress Distribution and Design Data for Adhesive Lap Joints Between Circular Tubes', Transactions of ASME, Vol. 78, August 1956, pp. 1213-1221.

86. Sancaktar, E and Lawry, P.O., ' A Photoelastic Study of the Stress Distribution in Adhesively Bonded Joints with Prebent Adherends', Journal of Adhesion, Vol. 11, No. 3, December 1980, pp. 233- 241.

87. Ishai, O., Peretz, D. and Gali, S., 'Direct Determination of Interlaminar Stresses in Polymeric Adhesive Layer', Experimental Mechanics, Vol. 17, No. 7, July 1977, pp. 265-270.

88. Allman, D.J., 'A Theory for Elastic Stresses in Adhesive Bonded Lap Joints', Quarterly Journal of Mechanics and Applied Mathematics, Vol. 30, Part. 4, November 1977, pp. 415-436.

89. Renton, W.J. and Vinson, J.R., 'The Efficient Design of Adhesive Bonded Joints', Journal of Adhesion, Vol. 7, No. 3, October 1975, pp. 175- 193.

90. Renton, W.J. and Vinson, J.R., 'Analysis of Adhesively Bonded Joints Between Panels of Composite Materials', Journal of Applied Mechanics, Vol. 44, No. 1. March 1977, pp. 101- 106. 
91. Adams, R.D. and Peppiatt, N.A., 'Effect of Poisson's Ratio Strains in Adherends on Stresses of an Idealized Lap Joint', Journal of Strain Analysis, Vol. 8, No. 2, April 1973, pp. 134-139.

92. Adams, R.D. and Peppiatt, N.A., 'Stress Analysis of Adhesive-Bonded Lap Joints', Journal of Strain Analysis, Vol. 9, No. 3, July 1974, pp. 185-196.

93. Kinloch, A.J., 'Adhesion and Adhesives : Science and Technology', Chapman and Hall, London, 1987, pp. 214-231.

94. Cooper, P.A. and Sawyer, J.W., 'A Critical Examination of Stresses in an Elastic Single Lap Joint', NASA Technical Paper 1507, September 1979.

95. CRC Handbook of Tables for Applied Engineering Science, Bolz, R.E. and Tuve, G.L., Ed., 2nd Ed., CRC Press, Inc., Boca Raton, Florida, pp. 103,118,687.

96. Lees, S., Scott, R.F., Gaudert, P.C., Ubbink, W.H. and Poon, C., 'Characterization and Toughened Resin Testing of New Generation Carbon Fibre/Epoxy Systems', NRC-LTR-ST-1638, September 1987.

97. Krieger, R.B. Jr., American Cynamid Co., Bloomingdale Plant, Havre de Grace, Maryland, Private Communication.

98. Hart-Smith, L.J., 'Adhesive Bonded Single Lap Joints', NASA Contractor Report 112236, January 1973.

99. Adams, R.D., Coppendale, J. and Peppiatt, N.A., 'Failure Analysis of AluminiumAluminium Bonded Joints', Adhesion 2, Allen, K.W., Ed., Applied Science Publishers Ltd., London, 1978, pp. 105- 120.

100. ESDU, 'Shear Stresses in the Adhesives in Bonded Joints. Single Step Double Lap Joints Loaded in Tension', Engineering Sciences Data Unit Report No. 78042, London, 1978.

101. ESDU, 'Inelastic Shear Stresses and Strains in the Adhesives Bonding Lap Joints Loaded in Tension and Shear', Engineering Sciences Data Unit Report No. 79016, London, 1979.

102. Hart-Smith, L.J., 'Design of Adhesively Bonded Joints', Joining Fibre-reinforced Plastics, Matthews, F.L., Ed., Elsevier Applied Science, New York, 1987, pp. $271-$ 311. 
103. Hart-Smith, L.J., 'Designing to Minimize Peel Stresses in Adhesive-Bonded Joint', Delamination and Debonding of Materials, ASTM STP876, Johnson, W.S.. Ed.. American Society for Testing and Materials, Philadelphia, 1985, pp. 238-266.

104. Sturrock, W.R., 'Ultrasonic Evaluation of Graphite/Epoxy Composite Laminates', Proceedings of Conference on Advanced NDE Technology Sponsored by Industrial Materials Research Institute, Montreal, IGM 82-000-059, 1982, p. 37.

105. Wang, T.T., Ryan, F.W. and Schonhorn, H., 'Effect of Bonding Defects on Shear Strength in Tension of Lap Joints Having Brittle Adhesives', Journal of Applied Polymer Science, Vol. 16, No. 8, August 1972, pp. 1901-1909.

106. Foulkes, H., Shields, J., and Wake, W.C., 'The Variation of Bond Strength with Temperature : A preliminary Study of Metal-to-Metal Adhesion', Journal of Adhesion, Vol. 2, No., October 1970, pp. 254-269.

107. Krautkramer, J. and Krautkramer, H., ' Ultrasonic Testing of Materials', SpringerVerlag, New York, 2nd Ed., 1977.

108. Lewis, A., 'Adhesives and Adhesion Introductory Theoretical Concepts', Adhesives 1979-1980 Desk Top Data Bank, Cordura Publications Inc., California, p. iii.

109. Minford,J.D., 'Adhesives', Durability of Structural Adhesives, Kinloch, A.J., Ed., Applied Science Publishers, New York, 1983, pp.154-157.

110. Fahr, A., Lee, S., Tanary, S. and Haddad, Y., 'Determination of Strength in Adhesively Bonded Steel Specimens by Acousto-Ultrasonic Techniques', Submitted to Materials Evaluation for publication.

111. FM300 Adhesive Film, Data Bulletin BPT 242, American Cynamid Company, Engineered Materials Department, Aerospace Products, Wayne, New Jersey 07470.

112. Kausen, R.C., 'High and Low Temperature Adhesives Where Do We Stand? 'Materials Symposium, National SAMPE 7th, Los Angeles, 1964.

113. Vary, A., 'Concepts and Techniques for Ultrasonic Evaluation of Material Mechanical Properties', Mechanics of Nondestructive Testing, Stinchcomb, W.W., Ed., Plenum Publishing, New York, 1980, pp.123-141.

114. Bunton, L.G. et al, 'Investigation of Cure in Epoxy Resins : Ultrasonic and Thermally Stimulated Current Measurements', Journal of Applied Polymer Science, Vol.27, 1982, pp.4283-4294. 
115. Fahr, A., Chapman, C.E., Tanary, S., Charlesworth, A.M. and Farahbakhsh, B.. 'Nondestructive Inspection of Foam Core Aluminium Panels', LTR-ST- 1655, National Research Council Canada, December 1987.

116. Benak, T. and Poon, C., 'Impact Testing of Foam Core Aluminium Panels', LTRST-483, National Research Council Canada, November 1987.

117. Vary, A. and Bowles, K.J., 'Ultrasonic Evaluation of the Strength of Unidirectional Graphite/Polyimide Composites', Proceedings of the Eleventh Symposium on Nondestructive Evaluation, Southwest Research Institute, San Antonio, Texas, 1977, pp.242-258.

118. Hemann, J.H. and Baaklini, G.Y., 'The Effect of Stress on Ultrasonic Pulses in Fiber Reinforced Composites', SAMPE Journal, Vol.22, No.4, July- August 1986, pp.9-13.

119. DATA 6000 Universal Waveform Analyser User Manual, Data Precision Division of Analogic Corp., Electronics Av., Danvers, MA., pp. D5-2 to D5-12.

120. Monchalin, J.P. and Heon, R., 'Laser Ultrasonic Generation and Optical Detection with a Confocal Fabry-Perot Interferometer', Materials Evaluation, Vol.44, No.10, September 1986, pp.1231-1237.

121. Sarrafzadeh-Khoee, A., Kiernan, M.T., Duke, J.C.Jr. and Henneke II, E.G., 'A Study of the Stress Wave Factor Technique for the Nondestructive Evaluation of Composite Materials', NASA Contractor Report 4002, July 1986. 


\section{Appendix A}

\section{Equations of the Stress Distributions}

\section{in a Single Lap Joint}

In the Goland Reissner's second theoretical analysis, they derived the shear and peel stress distribution in the adhesive along the overlap given by [84] :

$$
\begin{aligned}
\tau= & \frac{T}{8 l}\left\{\delta^{\prime}(1+3 k) \frac{\cosh \left(\delta^{\prime} x / l\right)}{\sinh \delta^{\prime}}+3(1-k)\right\} \\
\sigma_{y}= & \frac{T t}{l^{2} R_{3}}\left\{\left(R_{2} \lambda^{2} \frac{k}{2}+\lambda k^{\prime} \cosh \lambda \cos \lambda\right) \cosh (\lambda x / l) \cos (\lambda x / l)+\right. \\
& \left.\left(R_{1}(\lambda)^{2} \frac{k}{2}+\lambda k^{\prime} \sinh \lambda \sin \lambda\right) \sinh (\lambda x / l) \sin (\lambda x / l)\right\}
\end{aligned}
$$

where

$$
\begin{aligned}
\delta^{\prime} & =\sqrt{\frac{8 G_{a}}{E t h}} \\
\lambda & =\gamma \frac{l}{t}
\end{aligned}
$$




$$
\begin{aligned}
\gamma & =\sqrt[4]{\frac{6 E_{a} t}{E h}} \\
R_{1} & =\cosh \lambda \sin \lambda+\sinh \lambda \cos \lambda \\
R_{2} & =\sinh \lambda \cos \lambda-\cosh \lambda \sin \lambda \\
R_{3} & =\frac{1}{2}(\sinh (2 \lambda)+\sin (2 \lambda)) \\
k & =\frac{2 M}{T t} \\
& =\frac{\cosh \left(U_{2} l\right)}{\cosh \left(U_{2} l\right)+2 \sqrt{2} \sinh \left(U_{2} l\right)} \\
k^{\prime} & =\frac{U_{1} l}{2} k \\
U_{1} & =\sqrt{\frac{12 T\left(1-\mu^{2}\right)}{E t^{3}}} \\
U_{2} & =\sqrt{\frac{3 T\left(1-\mu^{2}\right)}{2 E t^{2}}}
\end{aligned}
$$

$M=$ bending moment

$E=$ Young's modulus of the adherends

$\mu=$ Poisson's ratio of the adherends

$E_{a}=$ Young's modulus of the adhesive

$G_{a}=$ Shear modulus of the adhesive.

Making some slight modifications of the Goland Reissner's theoretical analysis, Cooper and Sawyer [13] obtained the following shear and peel stress distributions :

$$
\frac{\tau}{\tau_{a v}}=\frac{1}{(4+3 \zeta)}\left\{\delta^{\prime \prime}\left(1+3 k_{1}\right) \frac{\cosh \left(\delta^{\prime \prime} x / l\right)}{\sinh \delta^{\prime \prime}}+3\left(1-k_{1}+\zeta\right)\right\}
$$

and

$$
\begin{aligned}
\frac{\sigma_{y}}{\sigma_{a p p}}= & \frac{t^{2}}{l^{2} R_{3}}\left\{\left(R_{2}\left(\lambda_{1}\right)^{2} \frac{k_{1}}{2}+\lambda_{1} k_{1}^{\prime} \cosh \lambda_{1} \cos \lambda_{1}\right) \cosh \left(\lambda_{1} x / l\right) \cos \left(\lambda_{1} x / l\right)+\right. \\
& \left.\left(R_{1}\left(\lambda_{1}\right)^{2} \frac{k_{1}}{2}+\lambda_{1} k_{1}^{\prime} \sinh \lambda_{1} \sin \lambda_{1}\right) \sinh \left(\lambda_{1} x / l\right) \sin \left(\lambda_{1} x / l\right)\right\}
\end{aligned}
$$


where

$$
\begin{aligned}
\tau_{a v} & =\frac{T_{o}}{2 l} \\
\sigma_{a p p} & =\frac{T_{o}}{t} \\
\delta^{\prime \prime} & =l \sqrt[4]{\frac{8 G_{a}\left(1-\mu^{2}\right)}{E t h}\left(1+\frac{3}{4} \zeta\right)} \\
\zeta & =\frac{h}{t} \\
\lambda_{1} & =l \sqrt[4]{\frac{E_{a}}{2 h D}}
\end{aligned}
$$

$D=$ the flexural stiffness of the adherend

$$
\begin{aligned}
D & =\frac{E t^{3}}{12\left(1-\mu^{2}\right)} \\
k_{1} & =\frac{2 M_{o}}{t T_{o}} \\
k_{1}^{\prime} & =\frac{l V_{o}}{t T_{o}}
\end{aligned}
$$

$T_{o}=$ internal axial force resultant

$$
=T \cos \phi
$$

$M_{o}=$ internal bending moment

$$
=T\left(\frac{t+h}{2}\right) \cos \phi \frac{U_{2}^{\prime} \cosh \left(U_{2}^{\prime} l\right) \sinh \left(U_{1}^{\prime} L\right)}{U_{2}^{\prime} \sinh \left(U_{1}^{\prime} L\right) \cosh \left(U_{2}^{\prime} l\right)+U_{1}^{\prime} \cosh \left(U_{1}^{\prime} L\right) \sinh \left(U_{2}^{\prime} l\right)}
$$

$V_{o}=$ internal shear force resultant

$$
=T\left(\frac{t+h}{2}\right) \cos \phi \frac{U_{1}^{\prime} U_{2}^{\prime} \cosh \left(U_{2}^{\prime} l\right) \cosh \left(U_{1}^{\prime} L\right)}{U_{2}^{\prime} \sinh \left(U_{1}^{\prime} L\right) \cosh \left(U_{2}^{\prime} l\right)+U_{1}^{\prime} \cosh \left(U_{1}^{\prime} L\right) \sinh \left(U_{2}^{\prime} l\right)}
$$




$$
\begin{aligned}
\cos \phi & =\frac{L+l}{\sqrt{(L+l)^{2}+\left(\frac{t+h}{2}\right)^{2}}} \\
U_{1}^{\prime} & =\sqrt{\frac{T \cos \phi}{D}} \\
U_{2}^{\prime} & =\sqrt{\frac{T \cos \phi}{8 D}}
\end{aligned}
$$




\section{APPENDIX B}

Computer Program for the Theoretical strength Prediction of Bonded Joints

The computer program is obtained from the work of Hart-Smith [98]. Some modifications on the form of the output have been made to suit the needs for the present study. The computer program is listed as follows:

C HART-SMITH PROGRAM

C SINGLE-LAP ADHESIVE BONDED JOINTS

C JOINT EFFICIENCY COMPUTATIONS

C DIMENSIONAL SOLUTION FOR BALANCED ADHERENDS

C PLASTIC HINGE FORMATION (OR FRACTURE) OF ADHERENDS INCLUDED

C POTENTIAL BOND SHEAR STRENGTH ACCOUNTED FOR SHORT OVERLAPS

C SHEAR STRENGTH USUALLY EXCEEDS PEEL STRENGTH FOR LONG OVERLAPS

C PEEL STRESS (OR INTERLAMINAR TENSION) FAILURES INCLUDED

IMPLICIT REAL*8 $(A-\mathrm{H}, \mathrm{O}-\mathrm{Z})$

IMPLICIT INTEGER* $4(I-N)$

DIMENSION THICKN $(20), \operatorname{OLOVTH}(20), \operatorname{STRGTH}(20,20), \operatorname{STRBND}(20,20)$,

1 STRGPL $(20,20), \operatorname{EFFNCY}(20), \operatorname{EFFBND}(20,20), \operatorname{EFFPL}(20,20)$,

$2 \operatorname{STORE}(20), \operatorname{TOVTH}(20), \operatorname{BNDSTR}(20,20), \operatorname{BNDEFF}(20,20), \operatorname{EKBNDG}(20)$ DOUBLE PRECISION XIL2, V28

C READ IN DATA

C READ IN ADHEREND AND ADHESIVE PROPERTIES

C NN . LE. 17 AND MM . LE. 11 READ $(5,10)$ NICNT, NN , MM

10 FORMAT (3I3) WRITE $(6,11)$ N1CNT

11 FORMAT (IH ,' MATERIALS COMBINATION $=1$, I2, $/$ )

C NICNT = NUMBER OF INPUT DATA SETS TO BE READ IN DO $660 \mathrm{~N} 2 \mathrm{CNT}=1, \mathrm{~N} 1 \mathrm{CNT}$

READ $(5,20)$ AKB, EYOUNG, SIGMAX, POISSN , ETRNSV

20 FORMAT (5E12.4)

WRITE $(6,21)$ AKB , EYOUNG, SIGMAX, POISSN , ETRNSV

21 FORMAT $(1 \mathrm{H}, '$ ADHEREND PROPERTIES : ', $/ 1,1 \mathrm{~Kb}=1, \mathrm{~F} 10.4,1$, 1 'E11 $=1$, E12.4, $/, '$ SIGMA MAX.=, E12.4, $/, '$ POISSON RATIO $=$ ' $2, \mathrm{~F} 10.4, /, \mathrm{E} 22=1, \mathrm{E} 12.4, / / 1$ READ $(5,30)$ G, GAMMAR, ETA, TAUMAX, PEELSG, EPEEL

30 FORMAT (5E12.4) WRITE $(6,31)$ G, GAMMAR, ETA, TAUMAX, PEELSG, EPEEL

31 FORMAT ( $1 \mathrm{H}, '$ ADHESIVE PROPERTIES $: 1, / /, 1 \mathrm{G}=1, \mathrm{E} 12.4,1$, 1 ' GAMMAR $=1$, F10.4, $/, 1$ THICKNESS $=1$, F10.4, $/$, TAUMAX $=1$, 2 E12.4, $/, 1$ PEEL STRENGTH $=1$, E12.4, $/, ' E=1, E 12.4, / /)$ IF (N2CNT . GT. 1) GO TO 70

C READ IN ADHEREND THICKNESS ARRAY $\operatorname{READ}(5,40) \quad(\operatorname{THICKN}(I), I=1, N N)$

40 FORMAT (1OF6.3)

C READ IN OVERLAP TO THICKNESS RATIO ARRAY $\operatorname{READ}(5,50) \quad$ (OLOVTH $(J), J=1, M M)$ 


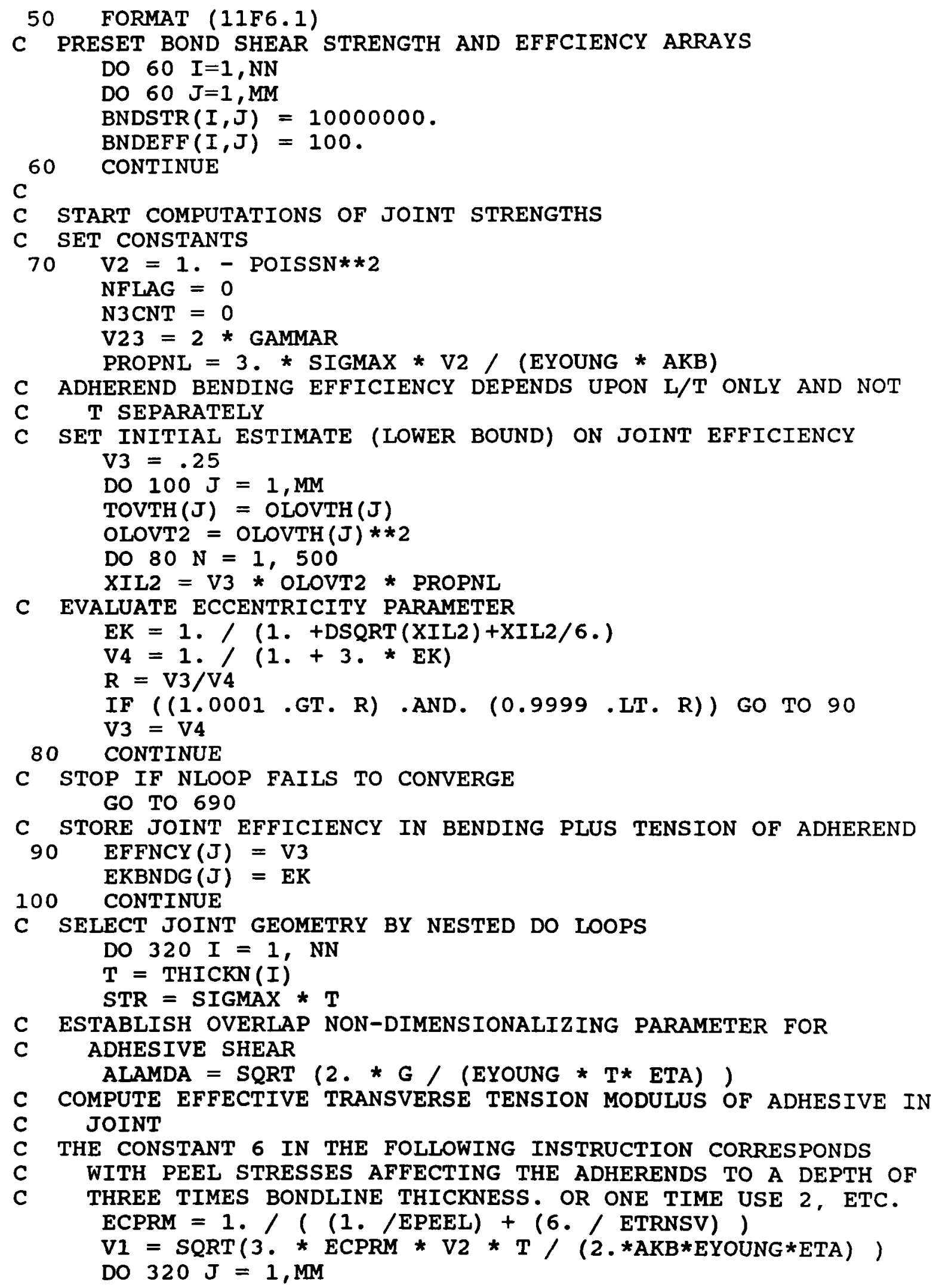




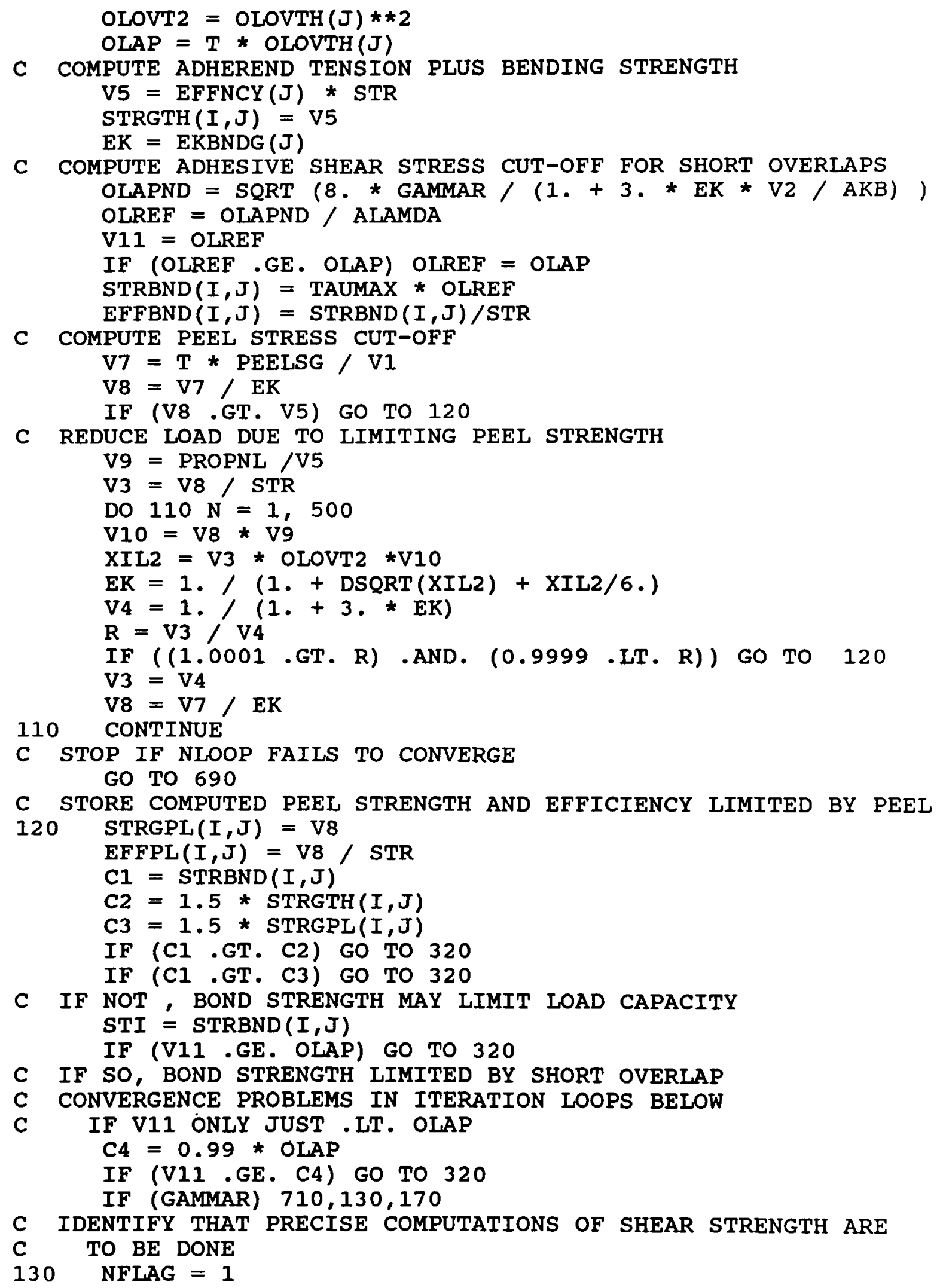




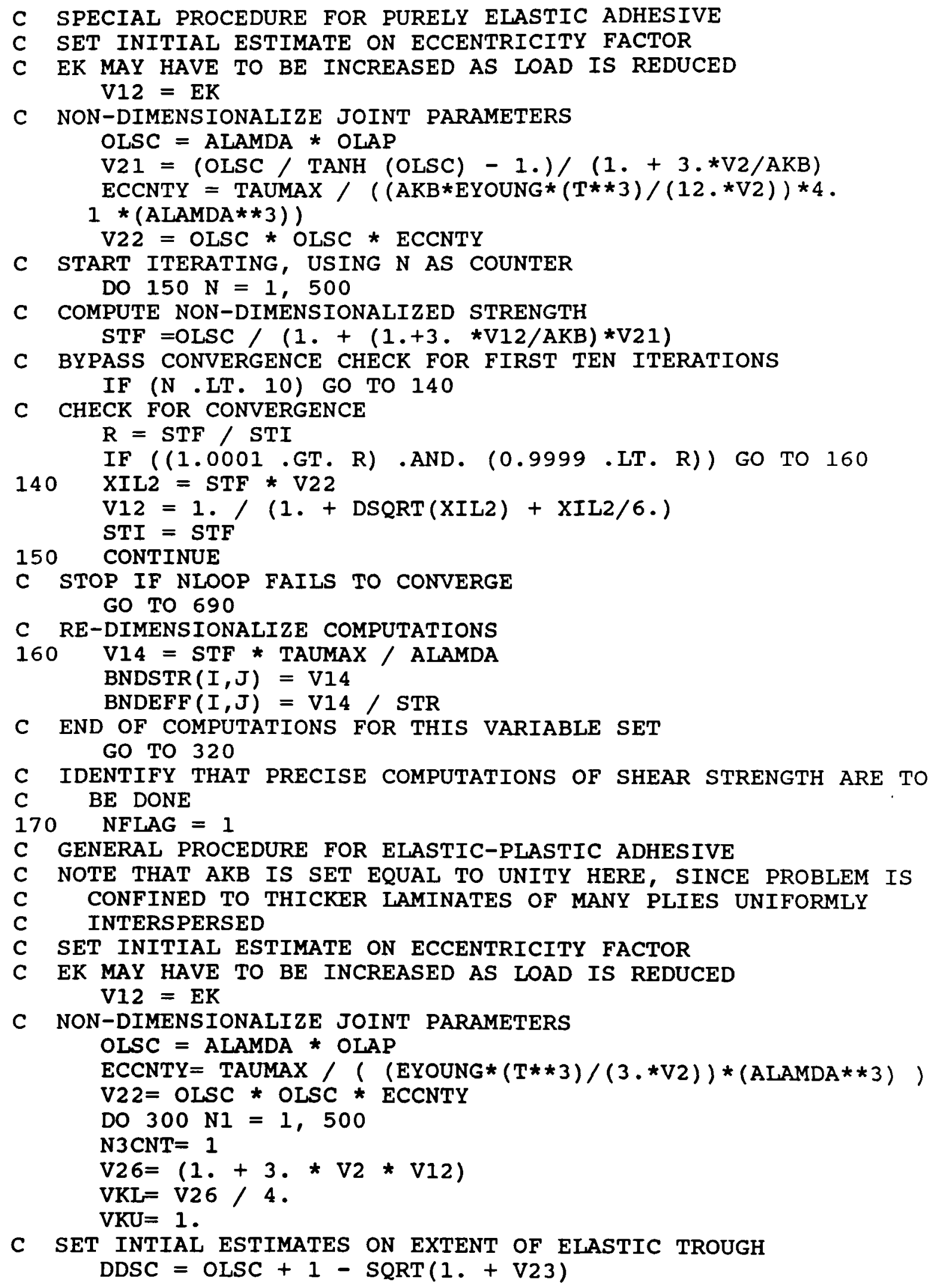




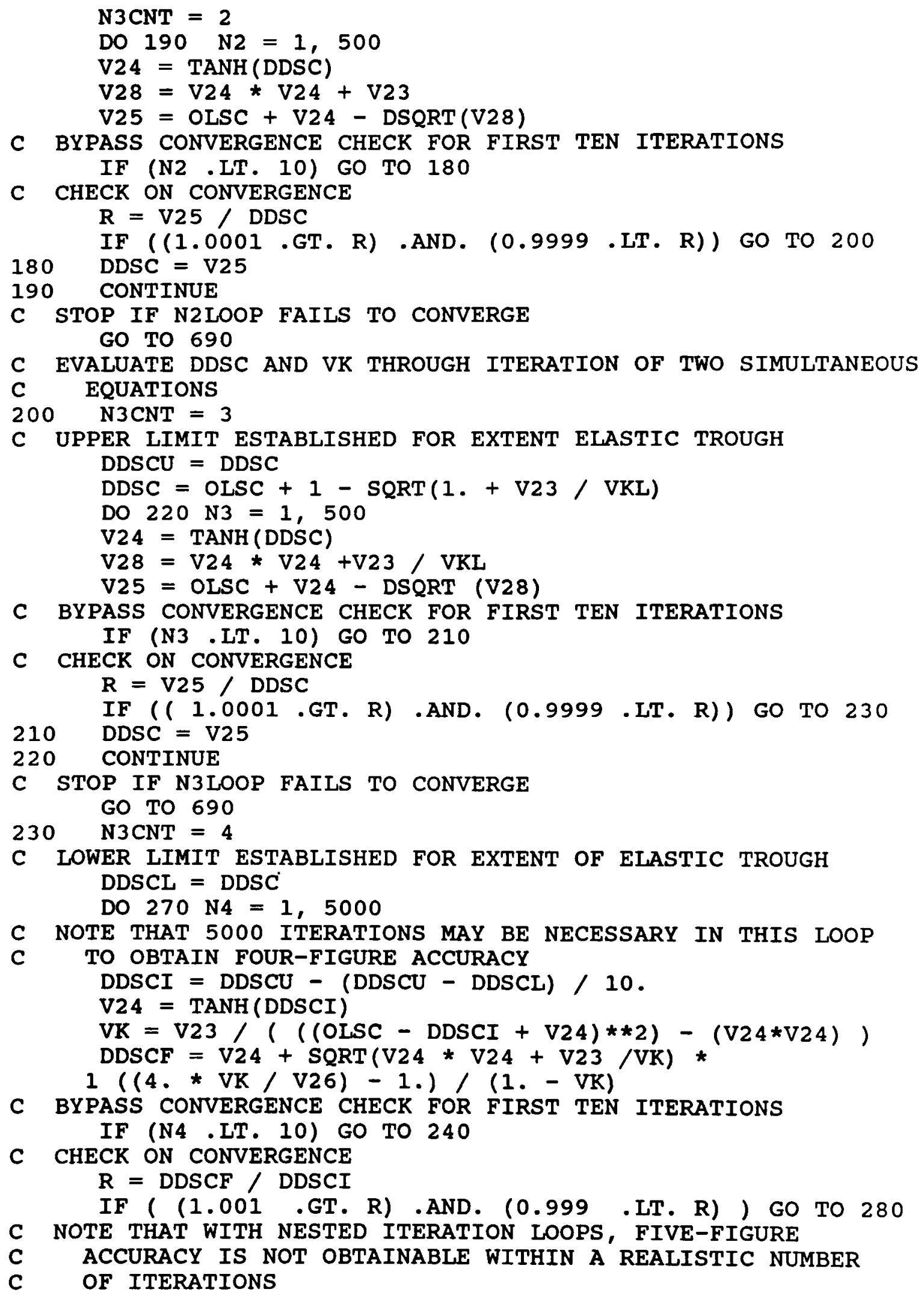




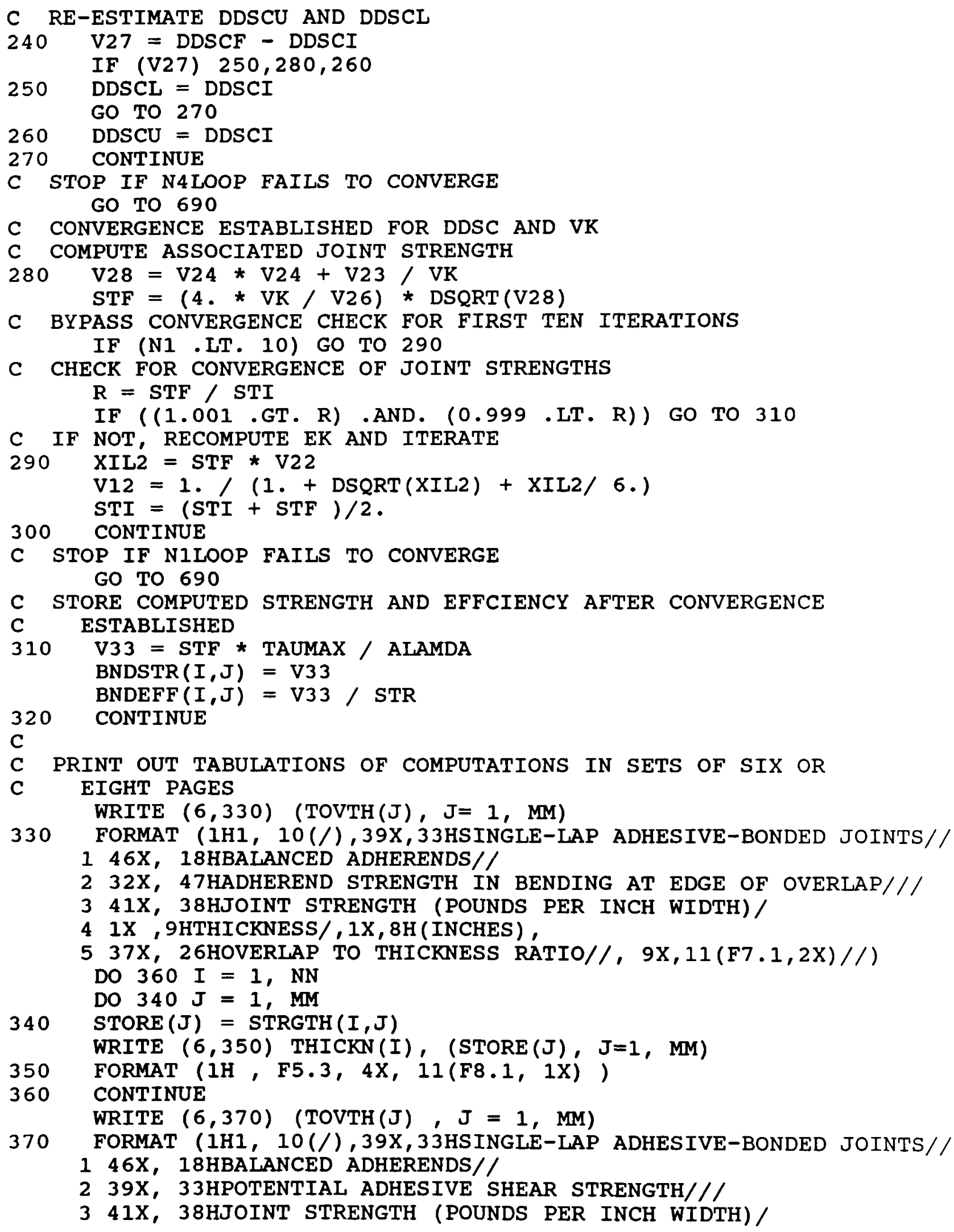


$41 \mathrm{X}, 9 H T H I C K N E S S /, 1 \mathrm{X}, 8 \mathrm{H}$ (INCHES),

$537 \mathrm{X}, 26$ HOVERLAP TO THICKNESS RATIO//,9X , 11(F7.1,2X)//)

DO $400 \mathrm{I}=1, \mathrm{NN}$

DO $380 \mathrm{~J}=1, \mathrm{MM}$

380

STORE $(J)=\operatorname{STRBND}(I, J)$

WRITE $(6,390) \operatorname{THICKN}(I),(\operatorname{STORE}(J), J=1, \operatorname{MM})$

IF (NFLAG . EQ. 0) GO TO 450

WRITE $(6,410)$ (TOVTH(J), J $=1, \mathrm{MM}$ )

410

FORMAT (1H1, $10(/), 39 \mathrm{X}, 33 \mathrm{HSINGLE-LAP} \mathrm{ADHESIVE-BONDED} \mathrm{JOINTS//}$

$146 \mathrm{X}, 18 \mathrm{HBALANCED}$ ADHERENDS//

$239 X, 33 H P O T E N T I A L$ ADHESIVE SHEAR STRENGTH///

$341 X, 38 H J O I N T$ STRENGTH (POUNDS PER INCH WIDTH)/

$41 \mathrm{X}, 9 \mathrm{HTHICKNESS} /, 1 \mathrm{X}, 8 \mathrm{H}$ (INCHES),

$537 \mathrm{X}, 26$ HOVERLAP TO THICKNESS RATIO//, 9X,11(F7.1,2X)//)

DO $440 I=1$, NN

DO $420 \mathrm{~J}=1, \mathrm{MM}$

420 STORE $(J)=\operatorname{BNDSTR}(I, J)$

WRITE $(6,430)$ THICKN (I), (STORE(J), J $=1$, MM)

430

440

450

460 FORMAT (1H , F5.3, 4X, 11(F8.1, 1X) )

CONTINUE

WRITE $(6,460) \quad($ TOVTH $(J), J=1$, MM)

FORMAT (1H1, $10(/), 39 \times, 33$ HSINGLE-LAP ADHESIVE-BONDED JOINTS//

$146 \mathrm{X}, 18 \mathrm{HBALANCED}$ ADHERENDS//

$244 \mathrm{X}, 22 \mathrm{HLIMITING}$ PEEL STRENGTH///

$341 \mathrm{X}$, 38HJOINT STRENGTH (POUNDS PER INCH WIDTH)/

$41 \mathrm{X}, 9 \mathrm{HTHICKNESS} /, 1 \mathrm{X}, 8 \mathrm{H}$ (INCHES),

$537 \mathrm{X}$, 26HOVERLAP TO THICKNESS RATIO//, 9X,11(F7.1,2X)//)

DO $490 \mathrm{I}=1, \mathrm{NN}$

DO $470 \mathrm{~J}=1, \mathrm{MM}$

470

$\operatorname{STORE}(J)=\operatorname{STRGPL}(I, J)$

WRITE $(6,480)$ THICKN(I), (STORE(J), J = 1, MM)

480

490

FORMAT (1H , F5.3, 4X, 11(F8.1, 1X) )

CONTINUE

WRITE $(6,500) \quad($ TOVTH $(J), J=1$, MM)

500 FORMAT (1H1, $10(/), 39 \mathrm{X}, 33 \mathrm{HSINGLE-LAP} \mathrm{ADHESIVE-BONDED} \mathrm{JOINTS//}$

$146 \mathrm{X}, 18 \mathrm{HBALANCED}$ ADHERENDS//

$232 \mathrm{X}, 47$ HADHEREND STRENGTH IN BENDING AT EDGE OF OVERLAP///

$352 \mathrm{X}$, 16HJOINT EFFICIENCY/

$41 \mathrm{X}, 9 \mathrm{HTHICKNESS} /, 1 \mathrm{X}, 8 \mathrm{H}$ (INCHES),

$537 \mathrm{X}$, 26HOVERLAP TO THICKNESS RATIO//, 9X,11(F7.1,2X)//)

DO $520 \mathrm{I}=1$, NN

WRITE $(6,510) \operatorname{THICKN}(I),(\operatorname{EFFNCY}(J), J=1, M M)$

510 FORMAT (1H , F5.3, 4X, 11(F8.5, 1X) )

520 CONTINUE

WRITE $(6,530) \quad($ TOVTH $(J), J=1, M M)$

530 FORMAT ( $1 \mathrm{H} 1,10(/), 39 \mathrm{X}, 33 \mathrm{HSINGLE-LAP} \mathrm{ADHESIVE-BONDED} \mathrm{JOINTS//}$

$146 \mathrm{X}, 18 \mathrm{HBALANCED}$ ADHERENDS//

2 39X, 33HPOTENTIAL ADHESIVE SHEAR STRENGTH///

3 52X, 16HJOINT EFFICIENCY/

$41 \mathrm{X}, 9 \mathrm{HTHICKNESS} /, 1 \mathrm{X}, 8 \mathrm{H}$ (INCHES), 
$537 X, 26 H O V E R L A P$ TO THICKNESS RATIO//, 9X,11(F7.1,2X)//)

DO $560 I=1, \mathrm{NN}$

DO $540 \mathrm{~J}=1, \mathrm{MM}$

540 STORE $(J)=$ EFFBND $(I, J)$

WRITE $(6,550) \operatorname{THICKN}(I),(\operatorname{STORE}(J), J=1, M M)$

550

FORMAT (1H , F5.3, 4X, 11(F8.5, 1X) )

560 CONTINUE

IF (NFLAG .EQ. O) GO TO 610

WRITE $(6,570)$ (TOVTH $(J), J=1$, MM)

570

FORMAT (1H1, 10(/),39X,33HSINGLE-LAP ADHESIVE-BONDED JOINTS//

$146 \mathrm{X}, 18 \mathrm{HBALANCED}$ ADHERENDS//

2 39X, 33HPOTENTIAL ADHESIVE SHEAR STRENGTH///

3 52X, 16HJOINT EFFICIENCY/

$41 \mathrm{X}, 9 \mathrm{HTHICKNESS} /, 1 \mathrm{X}, 8 \mathrm{H}$ (INCHES),

$537 \mathrm{X}, 26$ HOVERLAP TO THICKNESS RATIO//, 9X, 11(F7.1,2X)//)

DO $600 \mathrm{I}=1, \mathrm{NN}$

DO $580 \mathrm{~J}=1, \mathrm{MM}$

580 STORE $(J)=\operatorname{BNDEFF}(I, J)$

WRITE $(6,590)$ THICKN (I), (STORE (J), J = 1, MM)

590

600 FORMAT (1H , F5.3, 4X, 11(F8.5, 1X) )

CONTINUE

610

WRITE $(6,620)$ (TOVTH(J), J $=1$, MM)

620

FORMAT (1H1, $10(/), 39 \mathrm{X}, 33 \mathrm{HSINGLE}-\mathrm{LA}$

$146 \mathrm{X}, 18 \mathrm{HBALANCED}$ ADHERENDS//

$244 \mathrm{X}$, 22HLIMITING PEEL STRENGTH///

$352 \mathrm{X}, 16 \mathrm{HJOINT}$ EFFICIENCY/

$41 \mathrm{X}, 9$ HTHICKNESS/, $1 \mathrm{X}, 8 \mathrm{H}$ (INCHES),

$537 \mathrm{X}, 26$ HOVERLAP TO THICKNESS RATIO//, 9X,11(F7.1,2X)//)

DO $650 \mathrm{I}=1$, NN

DO $630 \mathrm{~J}=1, \mathrm{MM}$

$630 \operatorname{STORE}(\mathrm{J})=\operatorname{EFFPL}(\mathrm{I}, \mathrm{J})$

WRITE $(6,640)$ THICKN $(I), \quad(\operatorname{STORE}(J), J=1, \operatorname{MM})$

640 FORMAT (1H , F5.3, 4X, 11(F8.5, 1X) )

650 CONTINUE

c

C READ IN NEW MATERIAL ALLOWABLES AND REPEAT COMPUTATIONS

660 CONTINUE

670 WRITE $(6,680)$

680 FORMAT (1H1, 17HPROGRAM COMPLETED/)

STOP

C PRINT OUT DIAGNOSTICS

690 WRITE $(6,700)$ N3CNT, I, J

700 FORMAT (1H1, 19HDIVERGENT ITERATION, 3I10/) STOP

710 WRITE $(6,720)$

720 FORMAT (1H1, 'NEGATIVE GAMMAR VALUE IN INPUT DATA -

1 PHYSICALLY IMPOSSIBLE',/)

STOP

END 
Table 3.1 Reproducibility of acousto-ultrasonic data in terms of ring-down count (RDC) and peak amplitude (PA) measurements

\begin{tabular}{|c|c|c|}
\hline Trial No. & RDC & PA (dB) \\
\hline 1 & 39.0 & 85.1 \\
3 & 37.0 & 85.0 \\
4 & 40.0 & 85.5 \\
5 & 39.6 & 85.9 \\
6 & 36.5 & 86.0 \\
7 & 42.9 & 86.0 \\
8 & 36.0 & 85.0 \\
Mean & 38.0 & 85.1 \\
Standard & 38.625 & 85.450 \\
Deviation & 2.26 & 0.46 \\
& & \\
\hline
\end{tabular}


Table 4.1 Material properties used in theoretical analysis of stress distributions and strength predictions

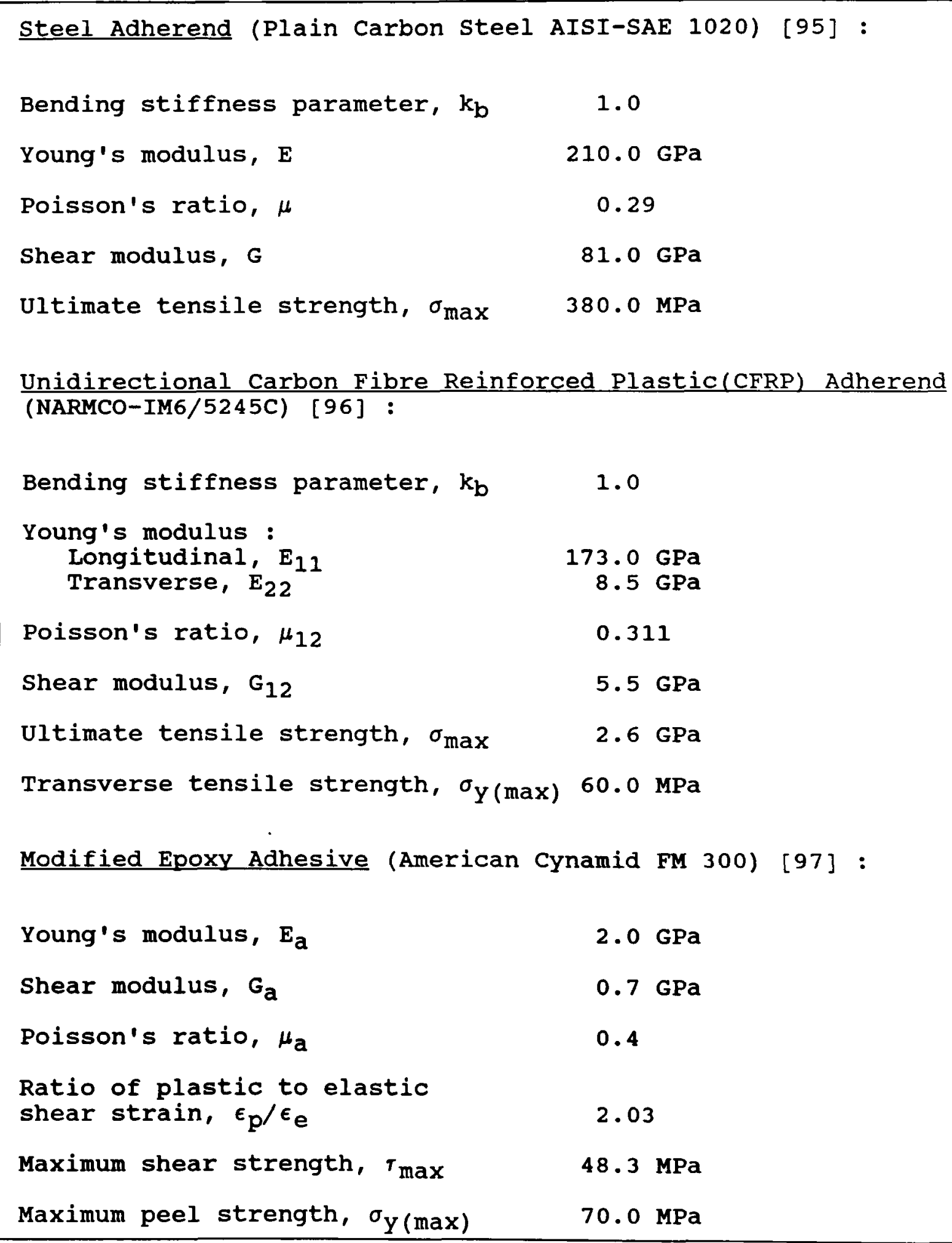


Table 5.1 Summary of the destructive testing results for specimens with voids

\begin{tabular}{|c|c|c|c|c|}
\hline $\begin{array}{l}\text { Specimen } \\
\text { No. }\end{array}$ & $\begin{array}{l}\text { Void* } \\
\text { size } \\
(z)\end{array}$ & $\begin{array}{l}\text { Type\# } \\
\text { of } \\
\text { bond }\end{array}$ & $\begin{array}{l}\text { Average } \\
\text { shear } \\
\text { strength } \\
\text { (MPa) }\end{array}$ & Failure Mode \\
\hline A1 & 2.50 & edge & 16.17 & $\begin{array}{l}\text { Predominantly cohesive failure } \\
\text { with some interfacial failure } \\
\text { around the small void area and } \\
\text { at the free edges. High } \\
\text { concentration of porosity } \\
\text { around the void area. }\end{array}$ \\
\hline A2 & 9.35 & cross & 16.74 & $\begin{array}{l}\text { Equally predominantly cohesive } \\
\text { and interfacial failure. } \\
\text { Interfacial failure occurring } \\
\text { at the free edges and around } \\
\text { the void area.High } \\
\text { concentration of porosity } \\
\text { around the void area. }\end{array}$ \\
\hline A3 & 9.37 & cross & 17.12 & Same as A2 (above). \\
\hline A4 & 0.00 & - & 17.87 & $\begin{array}{l}\text { Equally predominantly cohesive } \\
\text { and interfacial failure. } \\
\text { Interfacial failure occurring } \\
\text { at the free edges and in the } \\
\text { middle of the overlap length. }\end{array}$ \\
\hline A5 & 0.00 & - & 17.83 & $\begin{array}{l}\text { Predominantly cohesive failure } \\
\text { with some interfacial failure } \\
\text { at the free edges and at one } \\
\text { side of the joint area. }\end{array}$ \\
\hline B1 & 0.00 & - & 12.21 & $\begin{array}{l}\text { Predominantly fibre pull-off of } \\
\text { the surfaces of the adherends } \\
\text { with some cohesive failure in } \\
\text { the adhesive, and interfacial } \\
\text { failure at the free edges. }\end{array}$ \\
\hline B2 & 15.60 & edge & 4.29 & $\begin{array}{l}\text { Adherend cracked before the } \\
\text { adhesive. }\end{array}$ \\
\hline B3 & 23.20 & cross & 9.73 & $\begin{array}{l}\text { Same as A2 except with less } \\
\text { porosity. }\end{array}$ \\
\hline
\end{tabular}


Table 5.1 (Cont'd)

\begin{tabular}{|c|c|c|c|c|}
\hline $\begin{array}{c}\text { Specimen } \\
\text { No. }\end{array}$ & $\begin{array}{l}\text { Void* } \\
\text { size } \\
(\%)\end{array}$ & $\begin{array}{l}\text { Type \# } \\
\text { of } \\
\text { bond }\end{array}$ & $\begin{array}{l}\text { Average } \\
\text { shear } \\
\text { strength } \\
\text { (MPa) }\end{array}$ & Failure Mode \\
\hline B4 & 15.30 & across & 15.32 & $\begin{array}{l}\text { Same as } A 2 \text { except with less } \\
\text { porosity and with significant } \\
\text { fibre pull-off of the adherend } \\
\text { surfaces. }\end{array}$ \\
\hline B5 & 12.10 & across & 16.13 & Same as B4. \\
\hline B6 & 10.50 & across & 17.48 & Same as B4. \\
\hline B7 & 1.20 & edge & 17.50 & Same as B4. \\
\hline B8 & 0.00 & - & 17.90 & $\begin{array}{l}\text { Same as A5 except with some } \\
\text { minor fibre pull-off of the } \\
\text { adherend surfaces. }\end{array}$ \\
\hline B9 & 16.90 & edge & 12.51 & $\begin{array}{l}\text { Same as A2 except with less } \\
\text { porosity and with minor fibre } \\
\text { pull-off of the adherend } \\
\text { surfaces. }\end{array}$ \\
\hline B10 & 35.40 & across & 10.77 & Same as B3. \\
\hline B11 & 0.80 & edge & 15.31 & $\begin{array}{l}\text { Same as B8 except with small } \\
\text { void area. }\end{array}$ \\
\hline
\end{tabular}

* The ratio between the void area and the total overlap area.

\# For the location of the void areas, refer to the c-scan results in Figures 5.1 and 5.2

+ Fracture load divided by the total overlap area. 
Table 5.2 summary of the destructive testing results for specimens with unbonds

\begin{tabular}{|c|c|c|c|c|}
\hline $\begin{array}{l}\text { Specimen } \\
\text { No. }\end{array}$ & $\begin{array}{l}\text { Unbond } \\
\text { size } \\
\left(\mathrm{cm}^{2}\right)\end{array}$ & $\begin{array}{l}\text { Adherend } \\
\text { thickness } \\
\quad(\mathrm{cm})\end{array}$ & $\begin{array}{l}\text { Average } \\
\text { shear } \\
\text { strength } \\
\quad(\mathrm{MPa})\end{array}$ & Failure mode \\
\hline F1 & 0.40 & 0.320 & 24.76 & $\begin{array}{l}\text { Predominantly cohesive } \\
\text { failure with some } \\
\text { interfacial failure at } \\
\text { the free edges and with } \\
\text { fibre pull-off. }\end{array}$ \\
\hline F2 & 0.91 & 0.325 & 24.00 & $\begin{array}{l}\text { Predominantly } \\
\text { interlaminar failure } \\
\text { with some cohesive } \\
\text { failure and interfacial } \\
\text { failure at the free } \\
\text { edges. }\end{array}$ \\
\hline F3 & 1.61 & 0.351 & 29.78 & $\begin{array}{l}\text { Similar to Fl (above) } \\
\text { except with some inter- } \\
\text { laminar failure. }\end{array}$ \\
\hline F4 & 2.42 & 0.338 & 29.16 & Similar to F1. \\
\hline F5 & 1.61 & 0.351 & 25.43 & $\begin{array}{l}\text { Similar to F3 except } \\
\text { with more interlaminar } \\
\text { failure. }\end{array}$ \\
\hline F6 & 2.42 & 0.348 & 27.11 & similar to F3. \\
\hline F7 & 0.91 & 0.343 & 24.22 & similar to F5. \\
\hline F8 & 0.40 & 0.302 & 22.87 & Similar to F2. \\
\hline F9 & 0.00 & 0.353 & 33.33 & $\begin{array}{l}\text { Similar to F1 except } \\
\text { without unbond area. }\end{array}$ \\
\hline F10 & 0.00 & 0.353 & 28.27 & $\begin{array}{l}\text { Similar to F3 except } \\
\text { without unbond area. }\end{array}$ \\
\hline
\end{tabular}

+ Fracture load divided by the total overlap area. 
Table 6.1 Effect of cyclic thermal exposure on acoustoultrasonic parameter and average shear strength.

\begin{tabular}{|c|c|c|c|c|}
\hline \multirow[t]{2}{*}{$\begin{array}{l}\text { Spe- } \\
\text { cimen } \\
\text { no. }\end{array}$} & \multirow[t]{2}{*}{$\begin{array}{l}\text { Specimen } \\
\text { condition }\end{array}$} & \multirow[t]{2}{*}{$\begin{array}{l}\text { Normal- } \\
\text { ized } \\
\text { AUP } \\
\text { (sta. } \\
\text { dev.) }\end{array}$} & $\begin{array}{l}\text { Average shear } \\
\text { strength(test- } \\
\text { ed at room } \\
\text { temperature) }\end{array}$ & \multirow[t]{2}{*}{ Failure mode } \\
\hline & & & (MPa) & \\
\hline $\mathrm{T} 2$ & $\begin{array}{l}\text { Cyclic } \\
\text { Thermal } \\
\text { Exposure }\end{array}$ & $\begin{array}{l}0.755 \\
(0.031)\end{array}$ & 16.34 & $\begin{array}{l}\text { Predominantly interfa- } \\
\text { cial failure with some } \\
\text { cohesive failure and } \\
\text { thermal degradation } \\
\text { observed on some por- } \\
\text { tion of the adhesive. }\end{array}$ \\
\hline $\mathrm{T} 3$ & $\begin{array}{l}\text { Cyclic } \\
\text { Thermal } \\
\text { Exposure }\end{array}$ & $\begin{array}{l}0.830 \\
(0.040)\end{array}$ & 19.38 & $\begin{array}{l}\text { Equally predominant } \\
\text { cohesive and interfa- } \\
\text { cial failure and small } \\
\text { amount of thermal de- } \\
\text { gradation of the adhe- } \\
\text { sive observed at the } \\
\text { edges. }\end{array}$ \\
\hline $\mathrm{T} 4$ & $\begin{array}{l}\text { Cyclic } \\
\text { Thermal } \\
\text { Exposure }\end{array}$ & $\begin{array}{l}0.740 \\
(0.099)\end{array}$ & 16.72 & Same as T2. \\
\hline UO & $\begin{array}{l}\text { As- } \\
\text { fabricated }\end{array}$ & $\begin{array}{l}0.790 \\
(0.028)\end{array}$ & 20.14 & $\begin{array}{l}\text { Predominantly coh } \\
\text { failure with smal } \\
\text { amount of interfacial } \\
\text { failure. }\end{array}$ \\
\hline $\mathrm{UI}$ & $\begin{array}{l}\text { As- } \\
\text { fabricated }\end{array}$ & $\begin{array}{l}0.860 \\
(0.055)\end{array}$ & 20.90 & Same as above. \\
\hline $\mathrm{U} 2$ & $\begin{array}{l}\text { As- } \\
\text { fabricated }\end{array}$ & $\begin{array}{l}1.000 \\
(0.087)\end{array}$ & 24.70 & Same as above. \\
\hline
\end{tabular}


Table 7.1 Flaw types and description of flaw sites

\begin{tabular}{|c|c|c|}
\hline $\begin{array}{l}\text { Specimen } \\
\text { No. }\end{array}$ & $\begin{array}{l}\text { Flaw } \\
\text { Type }\end{array}$ & $\begin{array}{l}\text { Description of the } \\
\text { flaw site }\end{array}$ \\
\hline Fo/Al-1 & $\begin{array}{l}\text { A circular void ( } 25 \\
\text { mm in diameter) in } \\
\text { the adhesive layer. }\end{array}$ & Not visible at the surface \\
\hline $\begin{array}{l}\text { FO/Al-2 } \\
(11)\end{array}$ & $\begin{array}{l}\text { i) A } 20 \mathrm{~mm} \text { diameter } \\
\text { teflon tape on } \\
\text { one side. } \\
\text { ii) A large area of } \\
\text { fatigue damage } \\
\text { on the opposite } \\
\text { side. }\end{array}$ & $\begin{array}{l}\text { Not visible at the surface } \\
\text { Not visible at the surface, but } \\
\text { partly visible from the side }\end{array}$ \\
\hline $\begin{array}{l}\text { Fo/A1-3 } \\
(8705)\end{array}$ & $\begin{array}{l}\text { Using a } 12.5 \mathrm{~mm} \\
\text { diameter semi- } \\
\text { spherical head } \\
\text { indentor. } \\
\text { Three impact damage } \\
\text { sites on one side at: } \\
\text { i) } 1.5 \text { joules } \\
\text { ii) } 2.9 \text { joules } \\
\text { ii) } 14 \text { joules }\end{array}$ & $\begin{array}{l}\text { A } 1.5 \mathrm{~mm} \text { deep dent, visible at } \\
\text { the surface } \\
\text { A } 2.25 \mathrm{~mm} \text { deep dent, visible } \\
\text { at the surface } \\
\text { A } 5.15 \mathrm{~mm} \text { deep dent, quite } \\
\text { visible at the surface. }\end{array}$ \\
\hline $\begin{array}{l}\text { FO/AI-4 } \\
(8752)\end{array}$ & $\begin{array}{l}\text { Using a } 22.5 \mathrm{~mm} \\
\text { diameter nearly } \\
\text { flat head indentor. } \\
\text { Three impact damage } \\
\text { sites on one side at: } \\
\text { i) } 1.4 \text { joules } \\
\text { ii) } 2.8 \text { joules } \\
\text { ii) } 13.9 \text { joules }\end{array}$ & $\begin{array}{l}\text { A } 0.3 \mathrm{~mm} \text { deep dent, not visible } \\
\text { at the surface } \\
\text { A } 0.65 \mathrm{~mm} \text { deep dent, barely } \\
\text { visible at the surface } \\
\text { A } 2.32 \mathrm{~mm} \text { deep dent, visible } \\
\text { at the surface. }\end{array}$ \\
\hline
\end{tabular}


Table 7.2 The AUP data and the destructive test results of the foam core aluminium panels

\begin{tabular}{|c|c|c|c|}
\hline $\begin{array}{l}\text { Specimen } \\
\text { Code } \\
\text { (surface } \\
\text { preparation } \\
\text { techniques) }\end{array}$ & $\begin{array}{l}\text { Normalized } \\
\text { AUP } \\
\text { (standard } \\
\text { deviation) }\end{array}$ & $\begin{array}{l}\text { Peel Torque } \\
\text { per unit width, } \\
\text { mm } \mathrm{kg} / \mathrm{mm} \\
\text { (standard } \\
\text { deviation) }\end{array}$ & Failure Mode \\
\hline $\mathrm{AR}^{+}$ & $\begin{array}{l}1.00 \\
(0.072)\end{array}$ & $\begin{array}{c}0.259 \\
(0.011)\end{array}$ & $\begin{array}{l}\text { Completely interfacial } \\
\text { failure }\end{array}$ \\
\hline OR & $\begin{array}{c}0.72 \\
(0.074)\end{array}$ & $\begin{array}{l}0.407 \\
(0.030)\end{array}$ & $\begin{array}{l}\text { Predominantly inter- } \\
\text { facial failure with } \\
\text { some cohesive failure } \\
\text { in the adhesive }\end{array}$ \\
\hline NR & $\begin{array}{l}0.63 \\
(0.055)\end{array}$ & $\begin{array}{c}0.438 \\
(0.020)\end{array}$ & $\begin{array}{l}\text { Similar to on except } \\
\text { with more cohesive } \\
\text { failure in the adhesive }\end{array}$ \\
\hline NR2 & $\begin{array}{c}0.32 \\
(0.005)\end{array}$ & $\begin{array}{c}0.901 \\
(0.079)\end{array}$ & $\begin{array}{l}\text { Similar to NR except } \\
\text { with even more cohesive } \\
\text { failure in the adhesive }\end{array}$ \\
\hline ARP & $\begin{array}{l}0.48 \\
(0.105)\end{array}$ & $\begin{array}{c}1.037 \\
(0.133)\end{array}$ & $\begin{array}{l}\text { Predominantly inter- } \\
\text { facial failure with } \\
\text { some cohesive failure } \\
\text { in the adhesive and } \\
\text { minor cohesive failure } \\
\text { in the foam }\end{array}$ \\
\hline ORP & $\begin{array}{l}0.43 \\
(0.087)\end{array}$ & $\begin{array}{c}0.971 \\
(0.060)\end{array}$ & Similar to NR2 \\
\hline NR2 P & $\begin{array}{c}0.29 \\
(0.028)\end{array}$ & $\begin{array}{l}1.139 \\
(0.023)\end{array}$ & similar to NR2 \\
\hline NRP & $\begin{array}{l}0.18 \\
(0.036)\end{array}$ & $\begin{array}{c}1.328 \\
(0.075)\end{array}$ & $\begin{array}{l}\text { Predominantly cohesive } \\
\text { failure in the adhesive } \\
\text { with some interfacial } \\
\text { failure }\end{array}$ \\
\hline
\end{tabular}

$+\mathrm{AR}=\mathrm{As}-\mathrm{received} ; \mathrm{OR}=$ old roller; $\mathrm{NR}=$ New roller; $\mathrm{NR2}=$ New roller with 2 layers of adhesive; ARP = As-received with priming; $O R P=$ old roller with priming; $N R 2 P=$ New roller with 2 layers of adhesive and priming; NRP $=$ New roller with priming. 
Table 8.1 Fracture loads of the composite and steel specimens

\begin{tabular}{|cc|}
\hline & $\begin{array}{c}\text { Single lap bonded joint specimen in } \\
\text { Graphite/epoxy composites }\end{array}$ \\
\hline $\begin{array}{c}\text { Specimen } \\
\text { No. }\end{array}$ & $\begin{array}{c}\text { Fracture } \\
\text { Load } \\
(\mathrm{kN})\end{array}$ \\
\hline C1 & 12.74 \\
C2 & 16.17 \\
C3 & 16.66 \\
C5 & 13.23 \\
C6 & 16.24 \\
C7 & 16.91 \\
& 15.44 \\
& \\
\hline & Single 1 ap bonded joint specimen in steel \\
Specimen & Foad \\
No. & (kN) \\
\hline S1 & 13.48 \\
S2 & 15.93 \\
S4 & 13.86 \\
S5 & 12.94 \\
& 12.99 \\
\hline
\end{tabular}




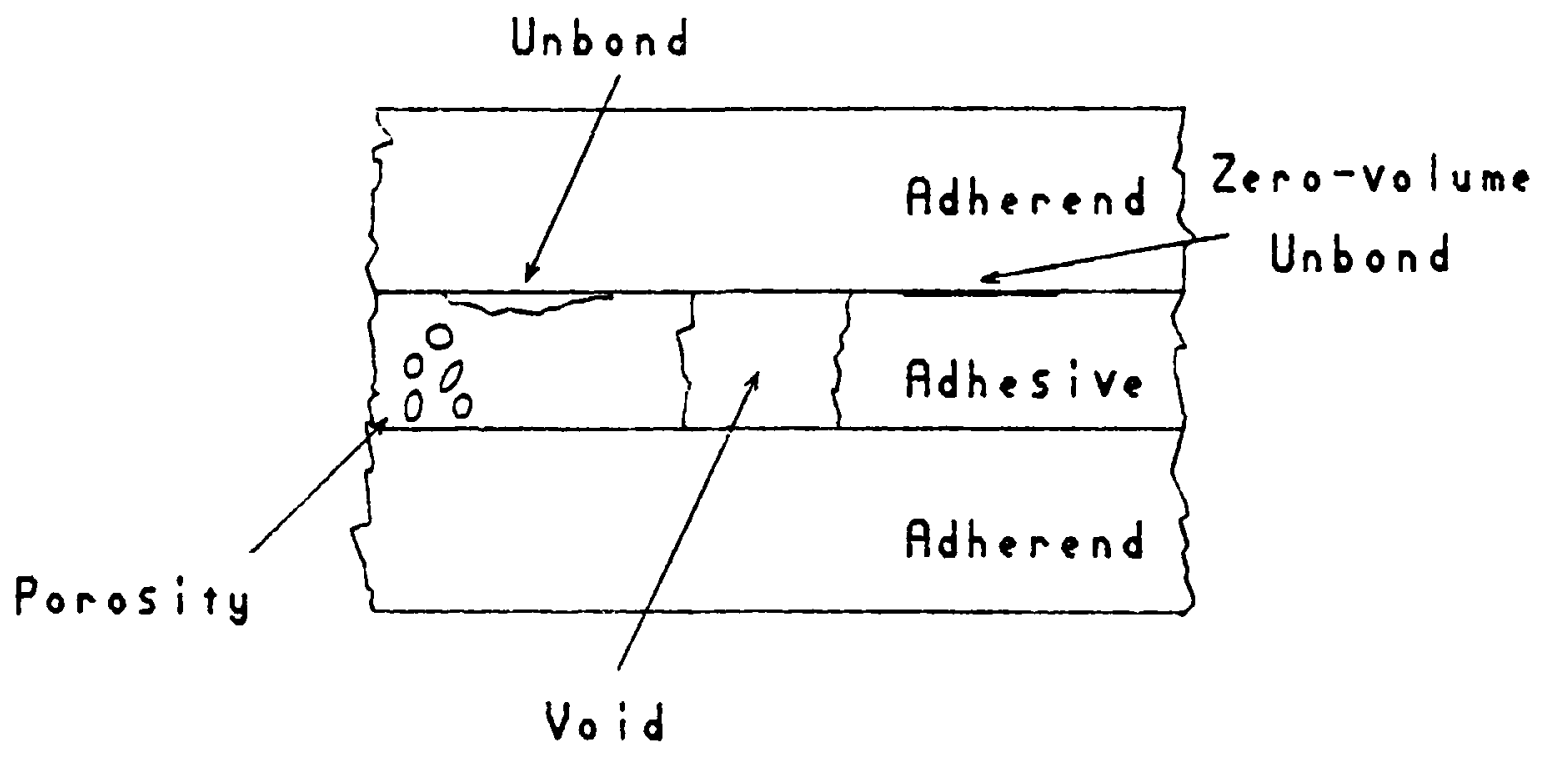

Figure 1.1: Physical discontinuities that may occur in an adhesively bonded joint 
Time

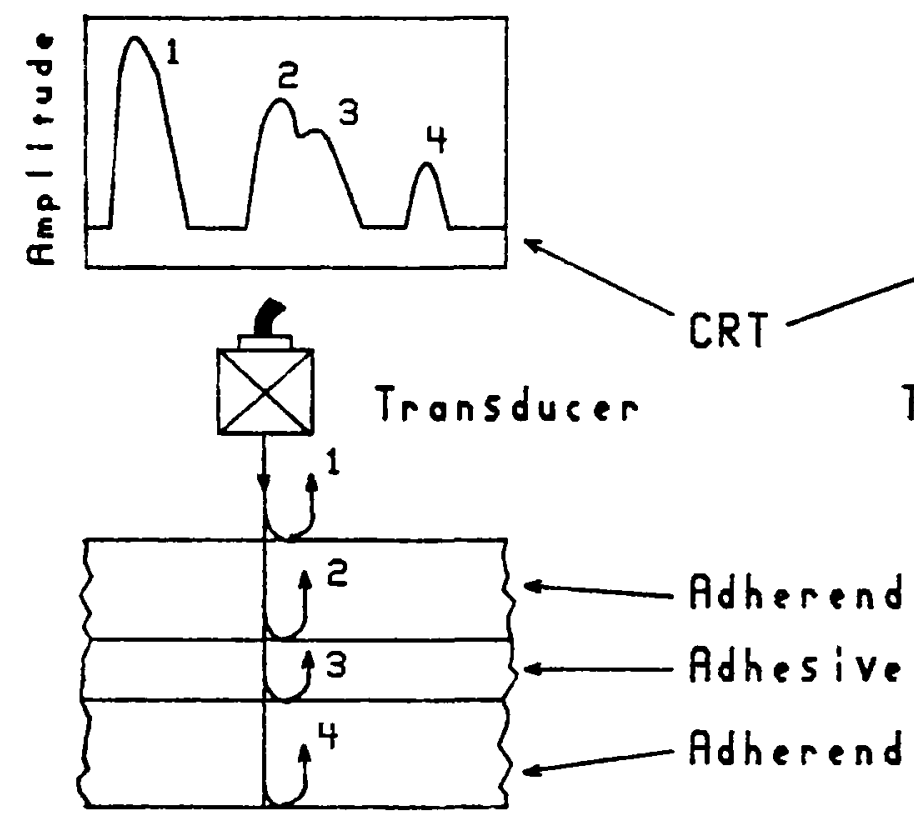

(a) Hithout Void
Time

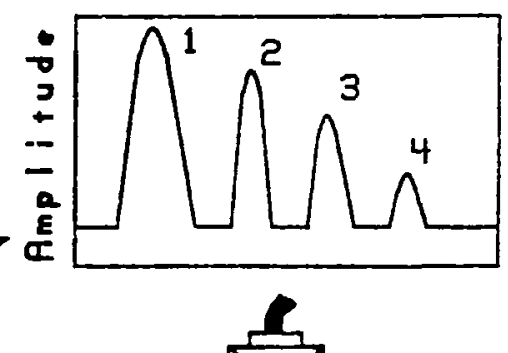

Tronsducer

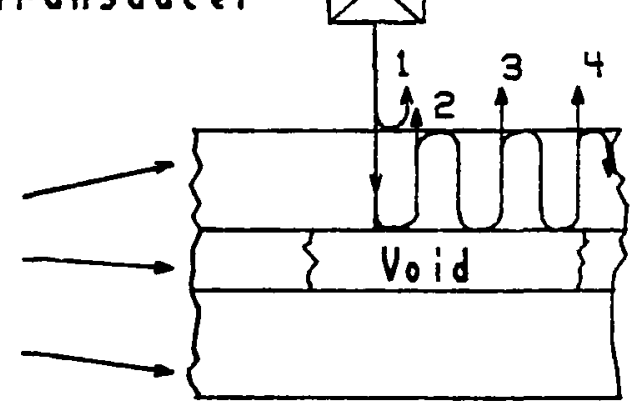

(b) With Void

Figure 2.1: Ultrasonic pulse echo technique 


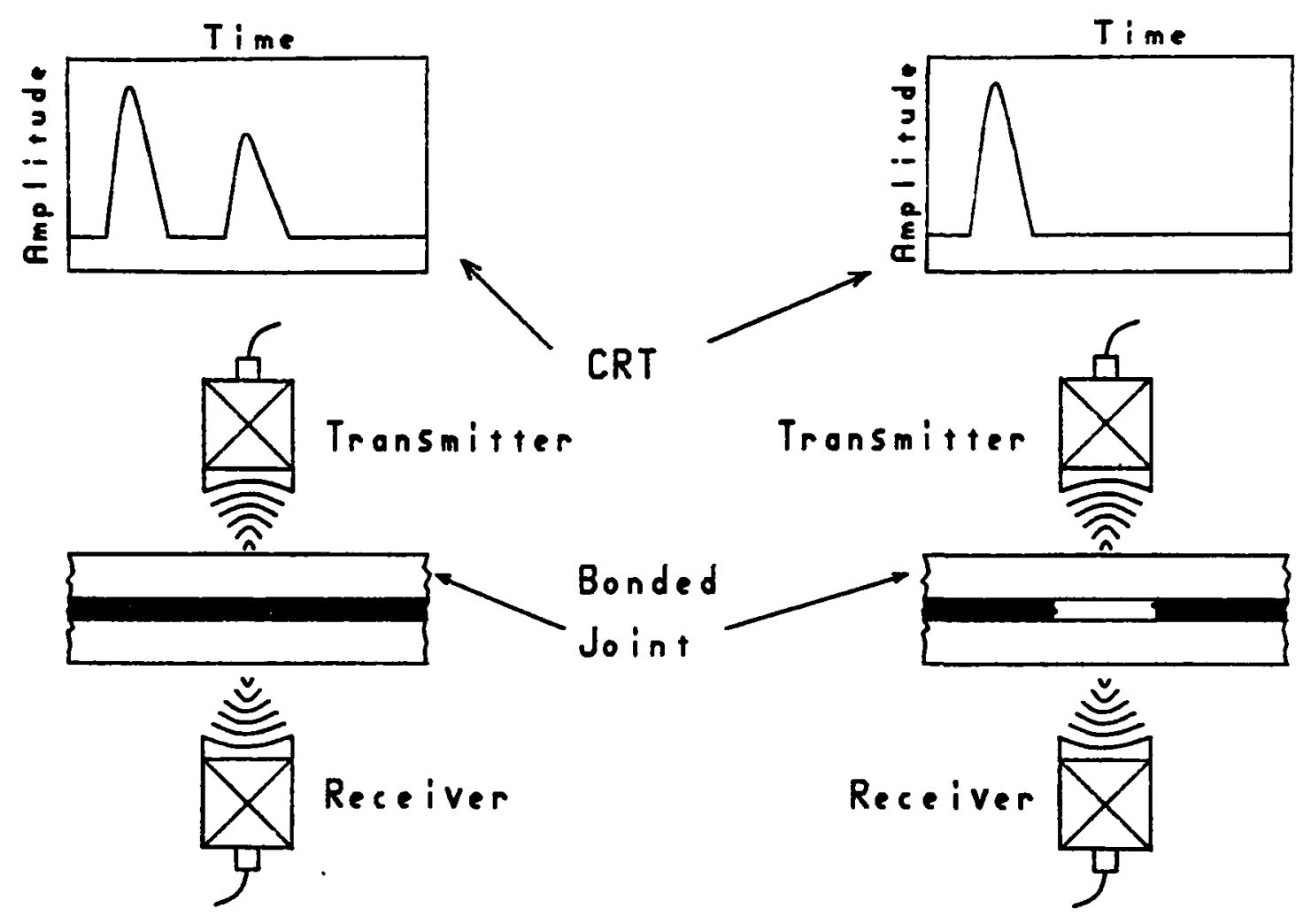

Figure 2.2 : Ultrasonic through-transmission technique 


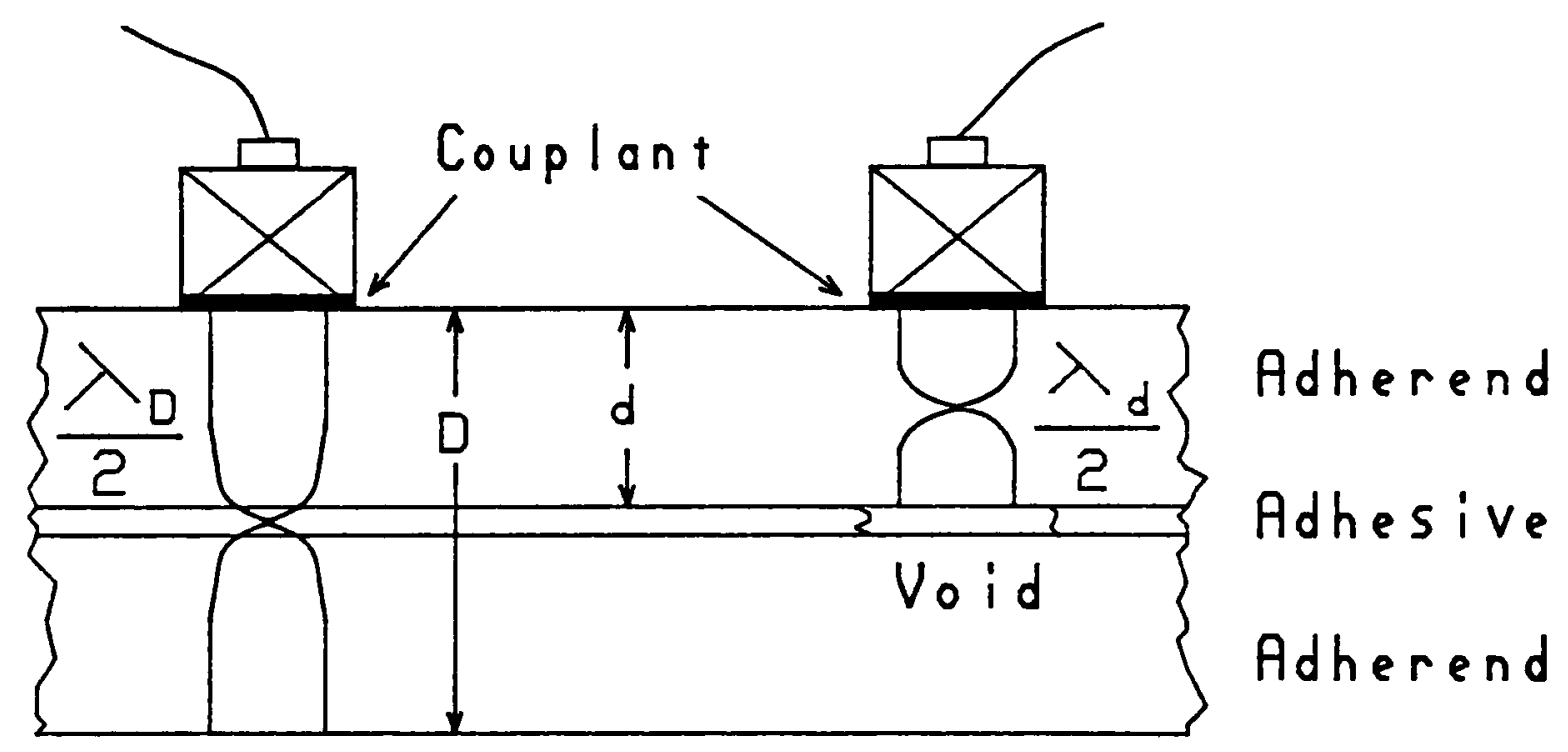

Figure 2.3 : Ultrasonic resonance method 


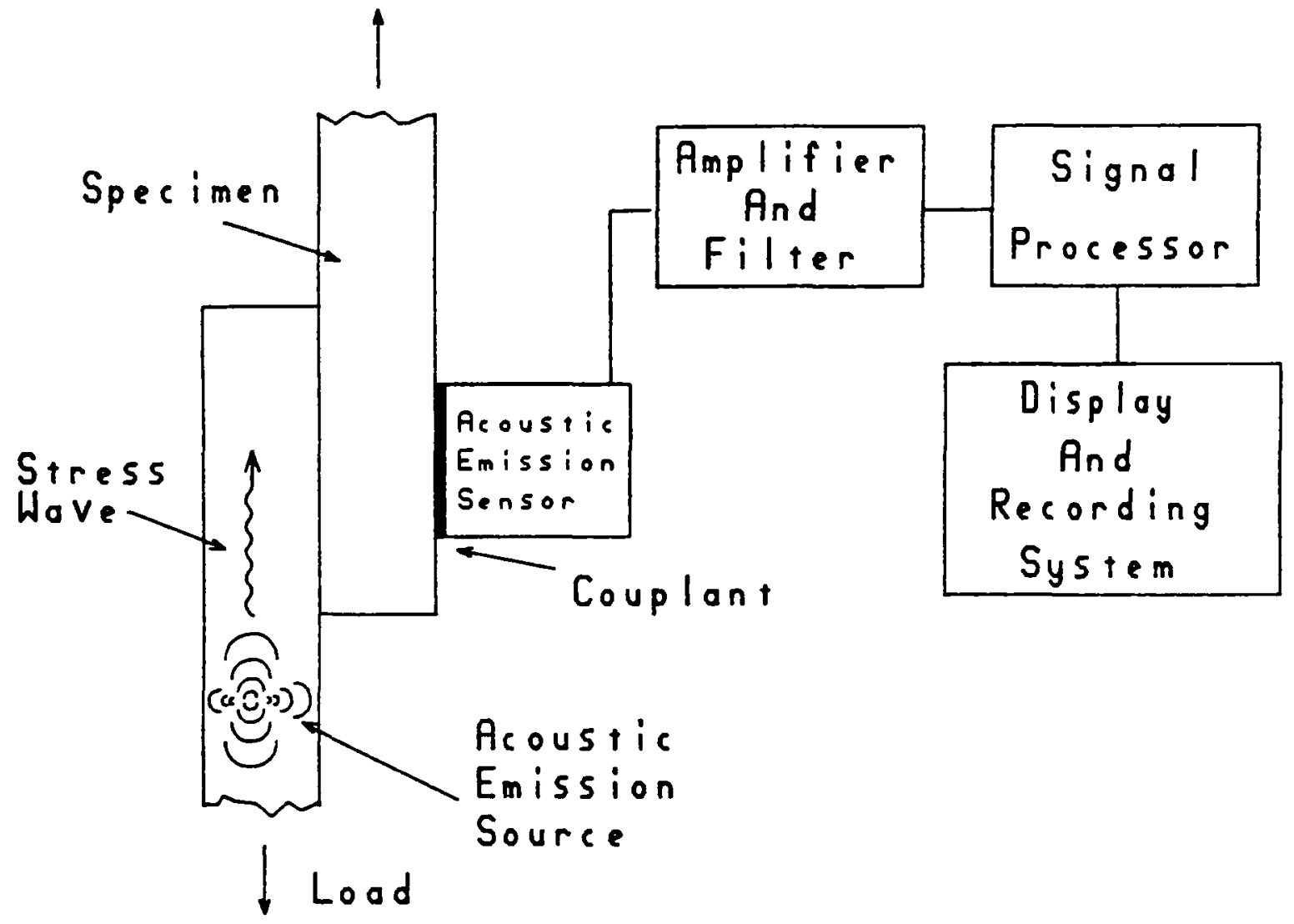

Figure 2.4: Simplified diagram of a typical acoustic emission system 


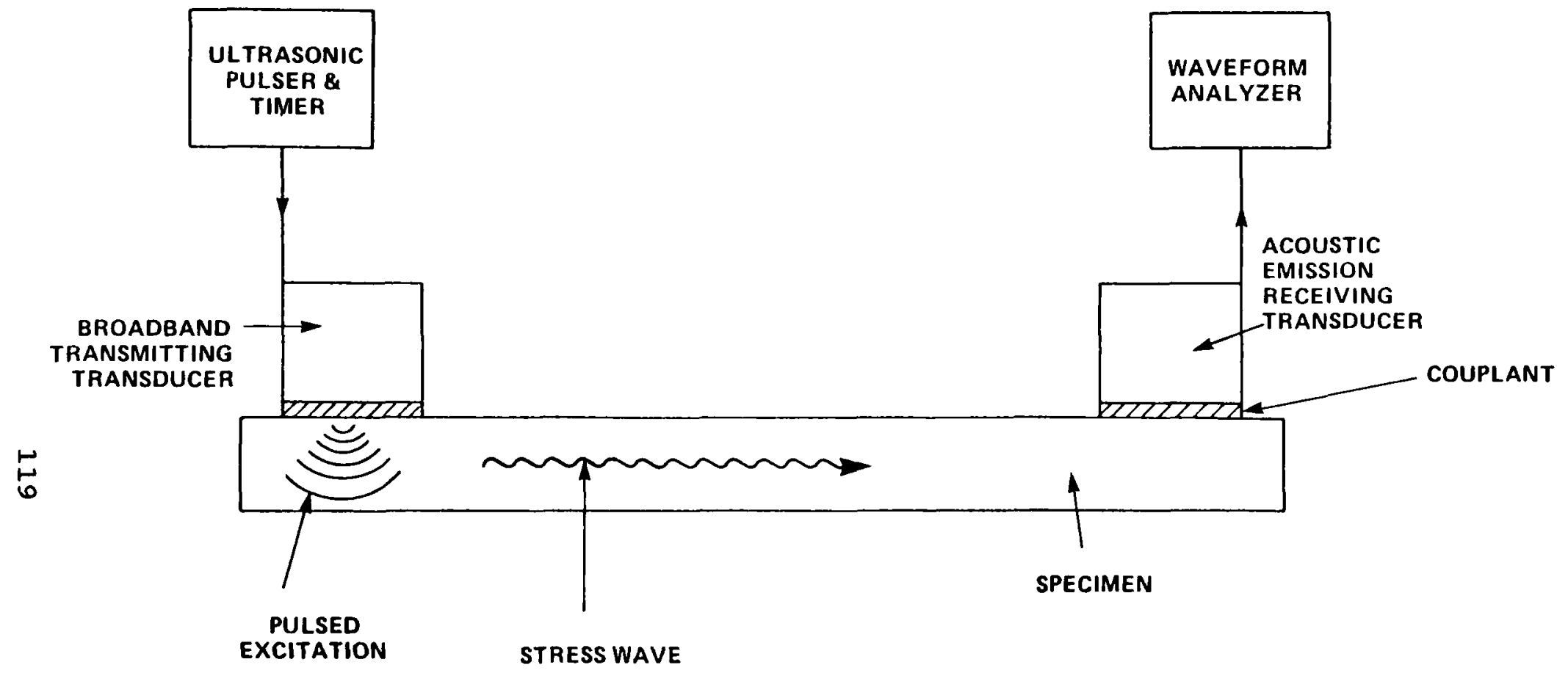

Figure 3.1 : A schematic diagram of the acousto-ultrasonic test set-up 


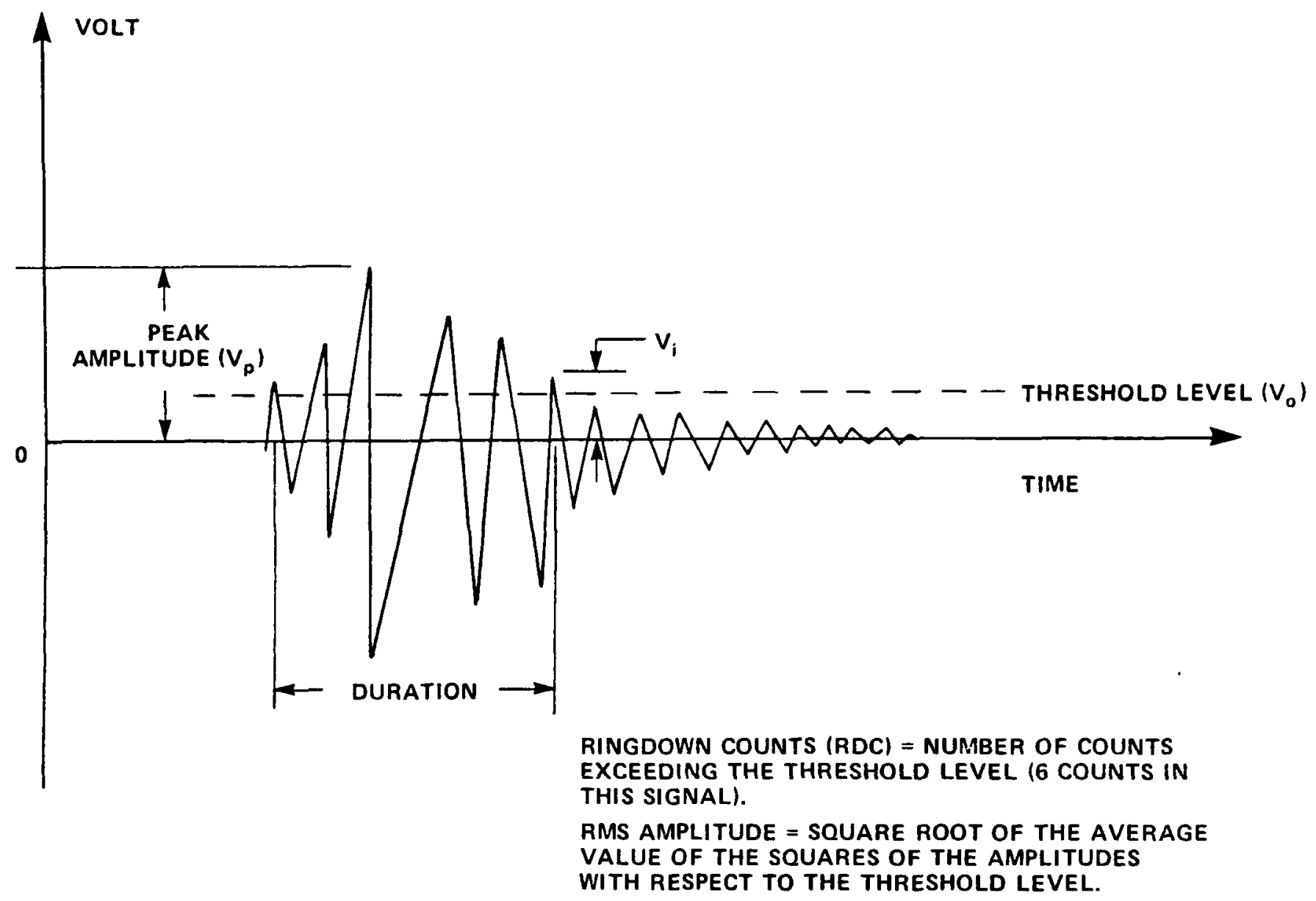

Figure 3.2 : Parameters measurable from an acousto-ultrasonic waveform 


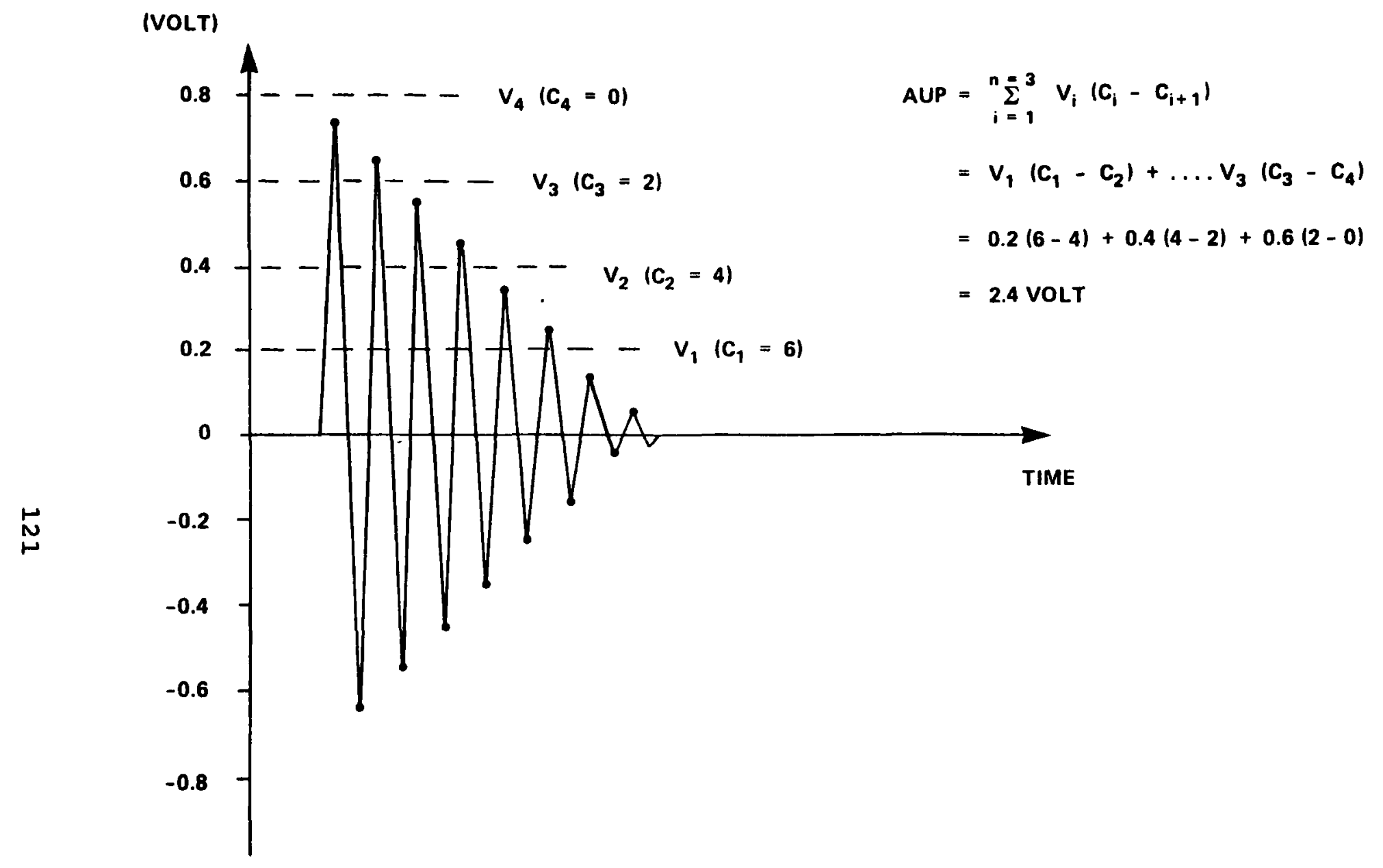

Figure 3.3 : An illustration of acousto-ultrasonic parameter (AUP) calculation 


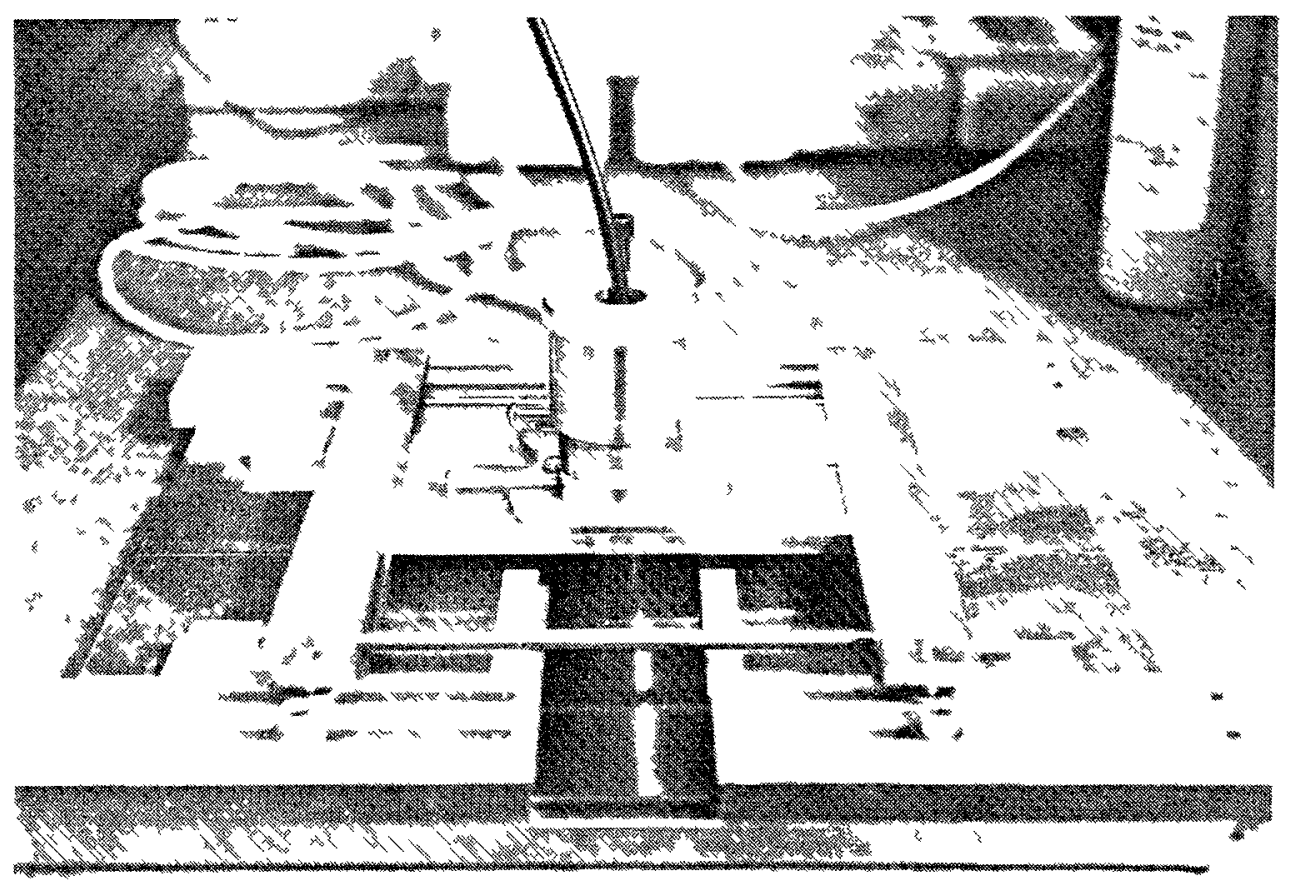

Figure 3.4 : The transducer-specimen holding fixture designed for improved reproducibility of $\mathrm{AU}$
measurement 


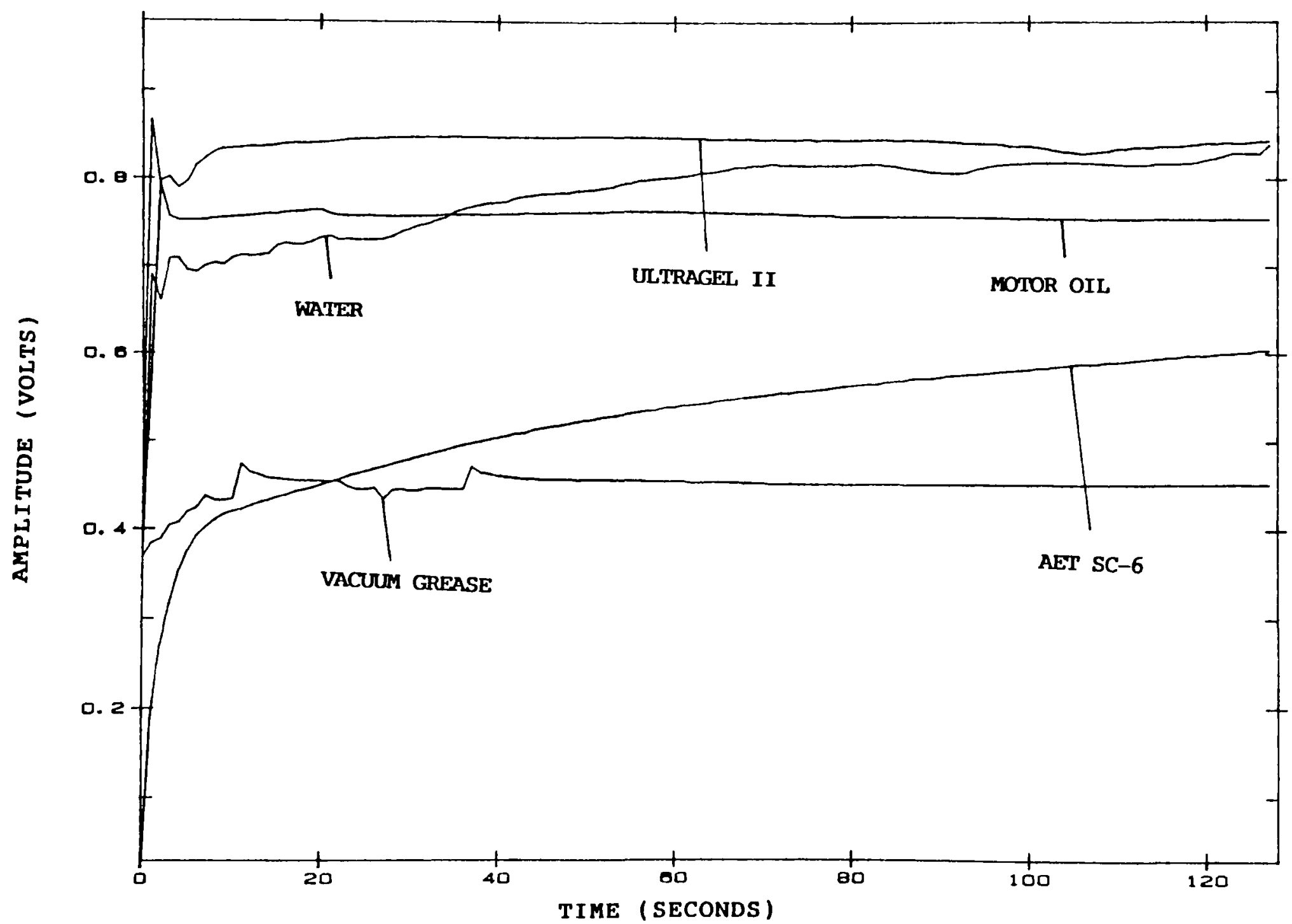

$\stackrel{\leftrightarrow}{\omega}$

Figure 3.5 : The change in AUP with respect to time for different coupling fluids used between the transducers and the specimen 

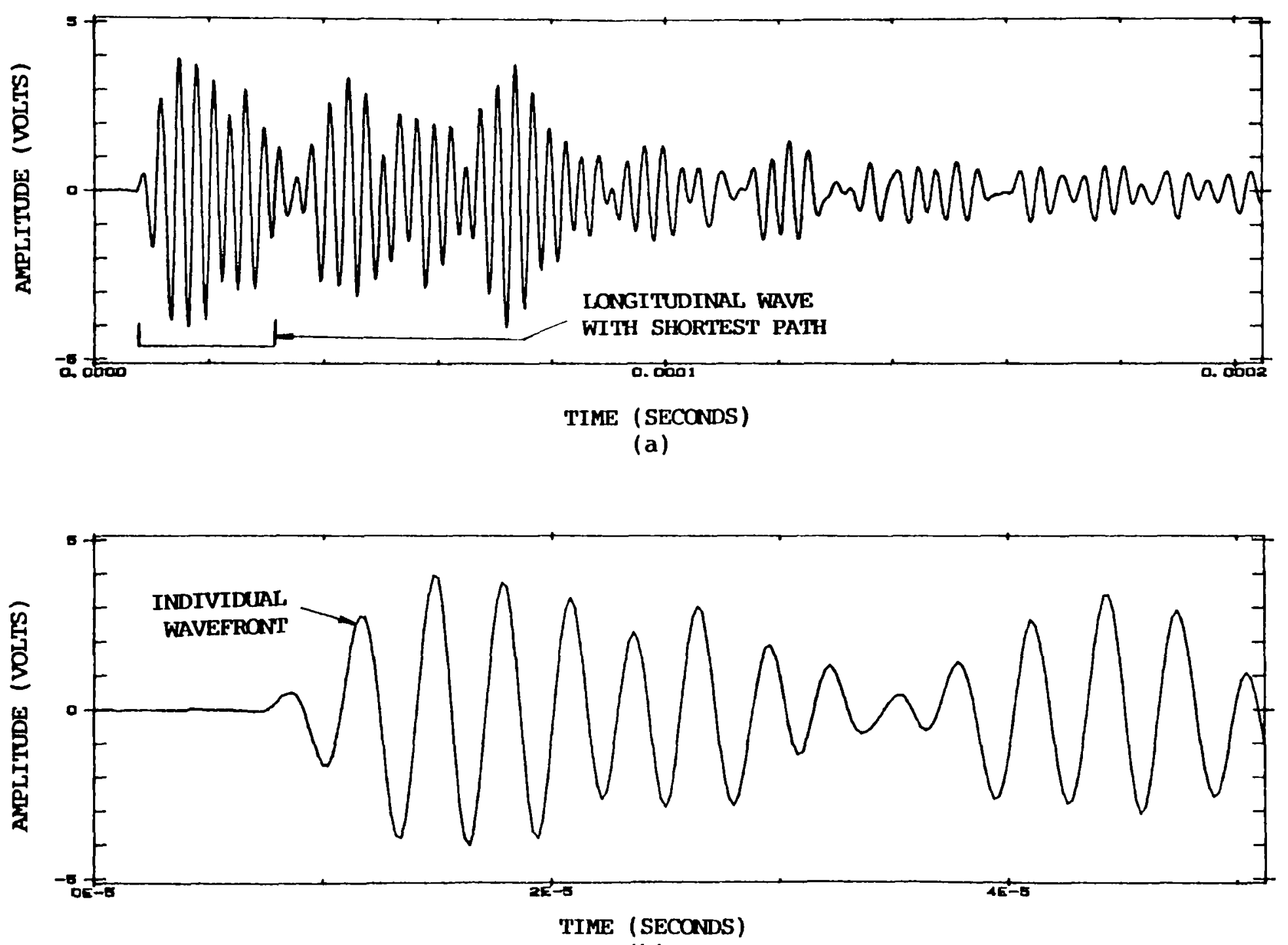

Figure 3.6 : (a) A typical waveform dbtained from a bonded joint in graphite/epoxy composites with a resonant condition; (b) Enlargement of the early portion of the waveform in (a) 


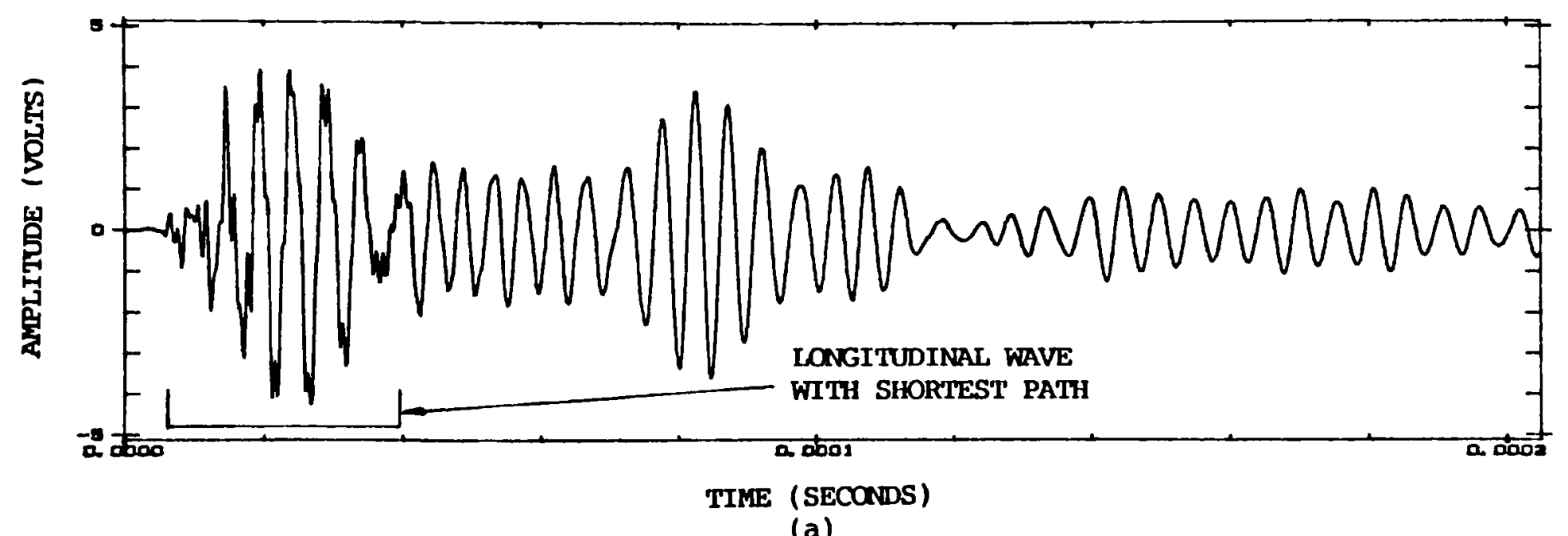

$\stackrel{\mathfrak{N}}{\mathfrak{N}}$

(a)

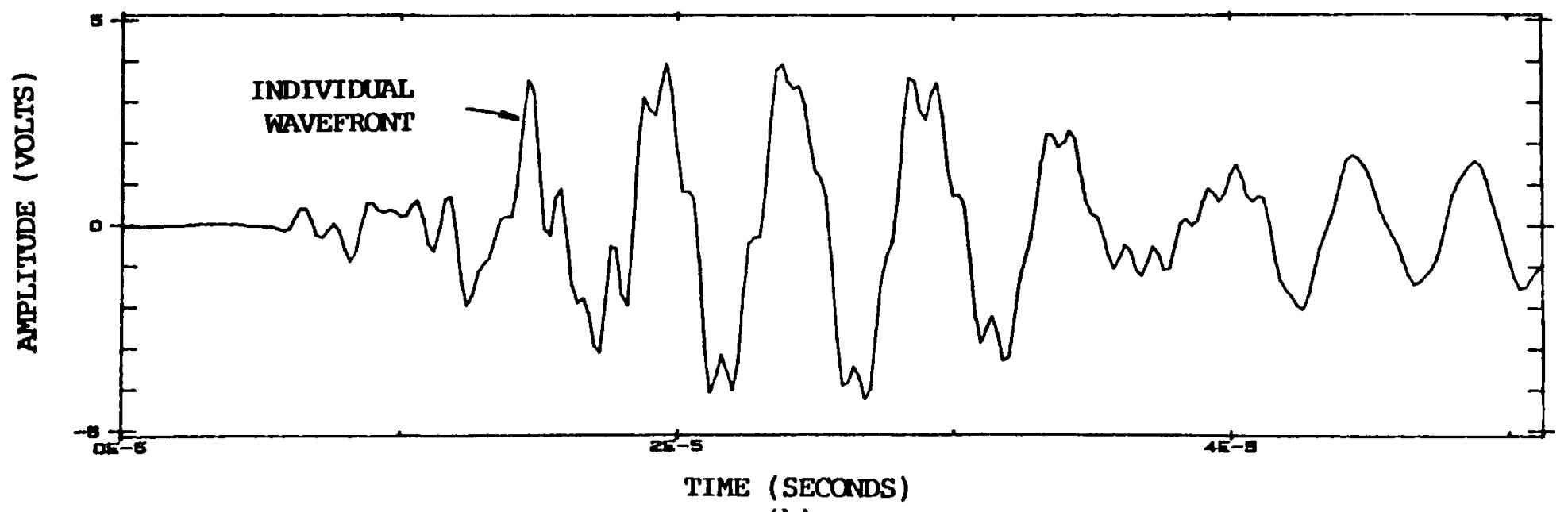

Figure 3.7 : (a) A typical waveform obtained from a bonded joint in graphite/epoxy composites with an off-resonant condition; (b) Enlargement of the early portion of the waveform in (a) 


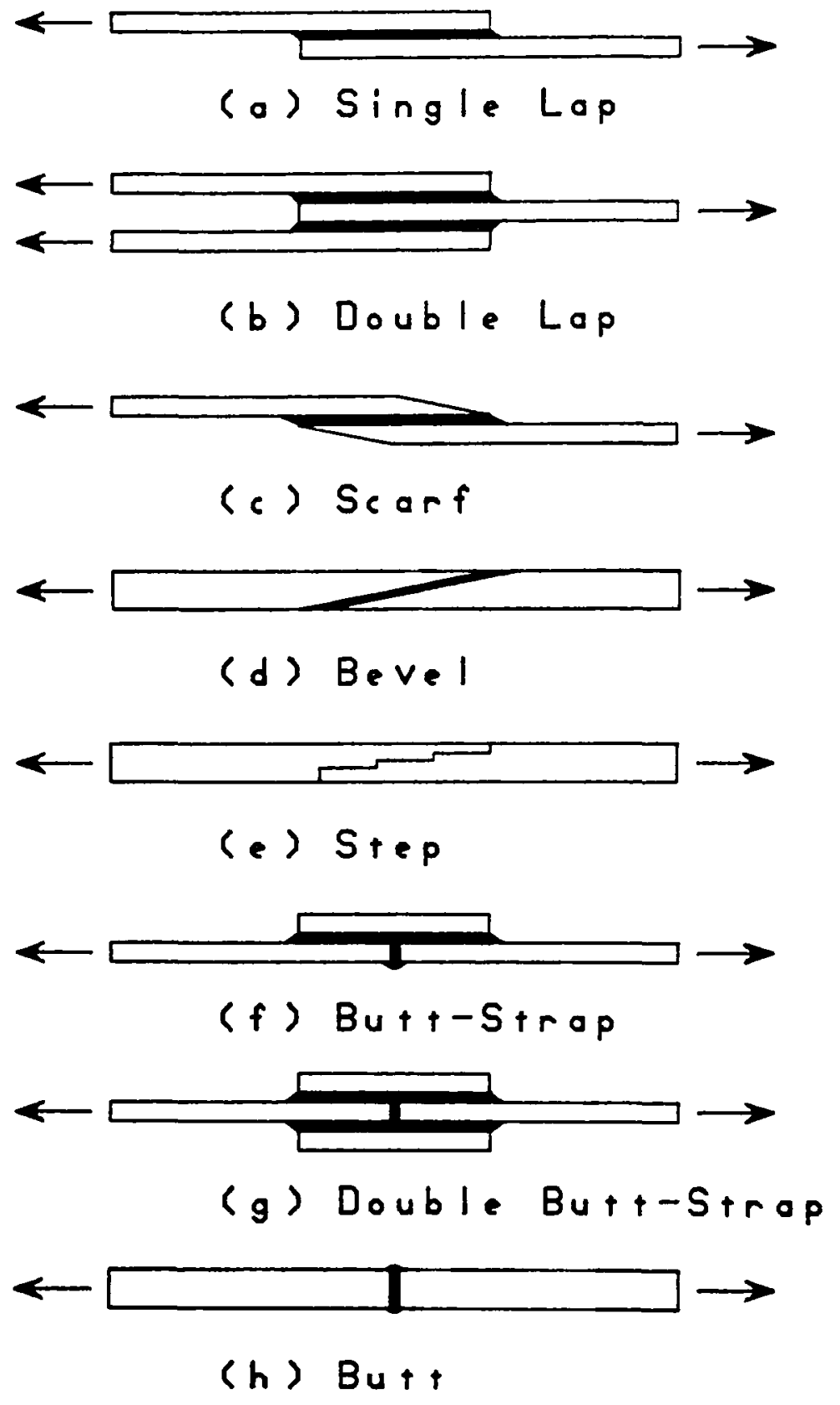

Figure 4.1 : Some typical joint configurations 


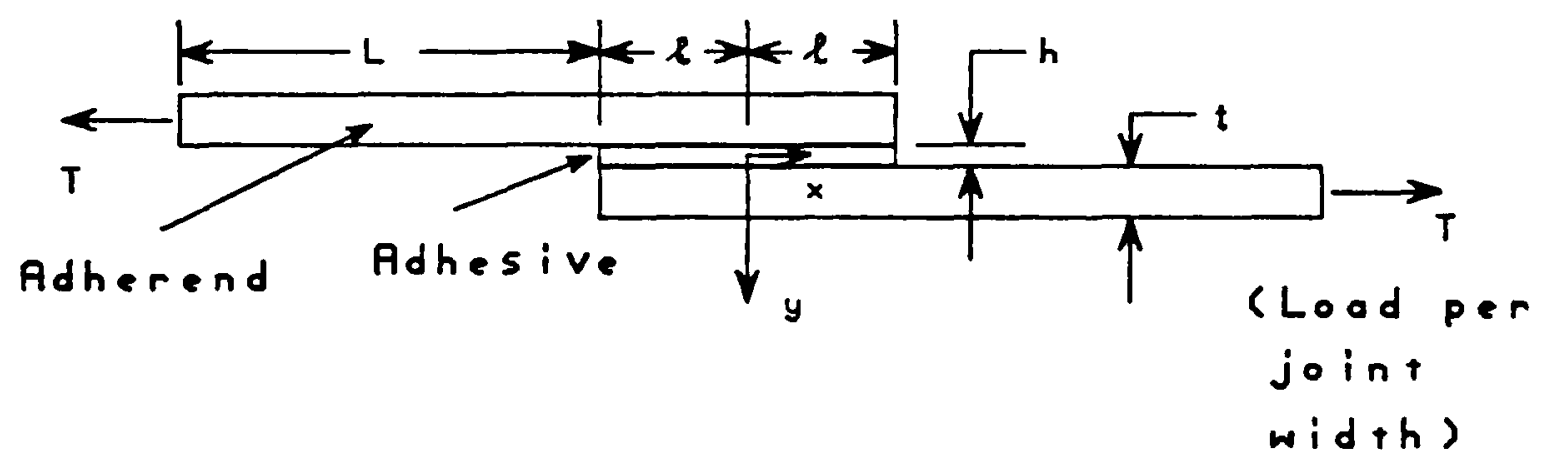

Figure 4.2 : Definitions of joint specimens 


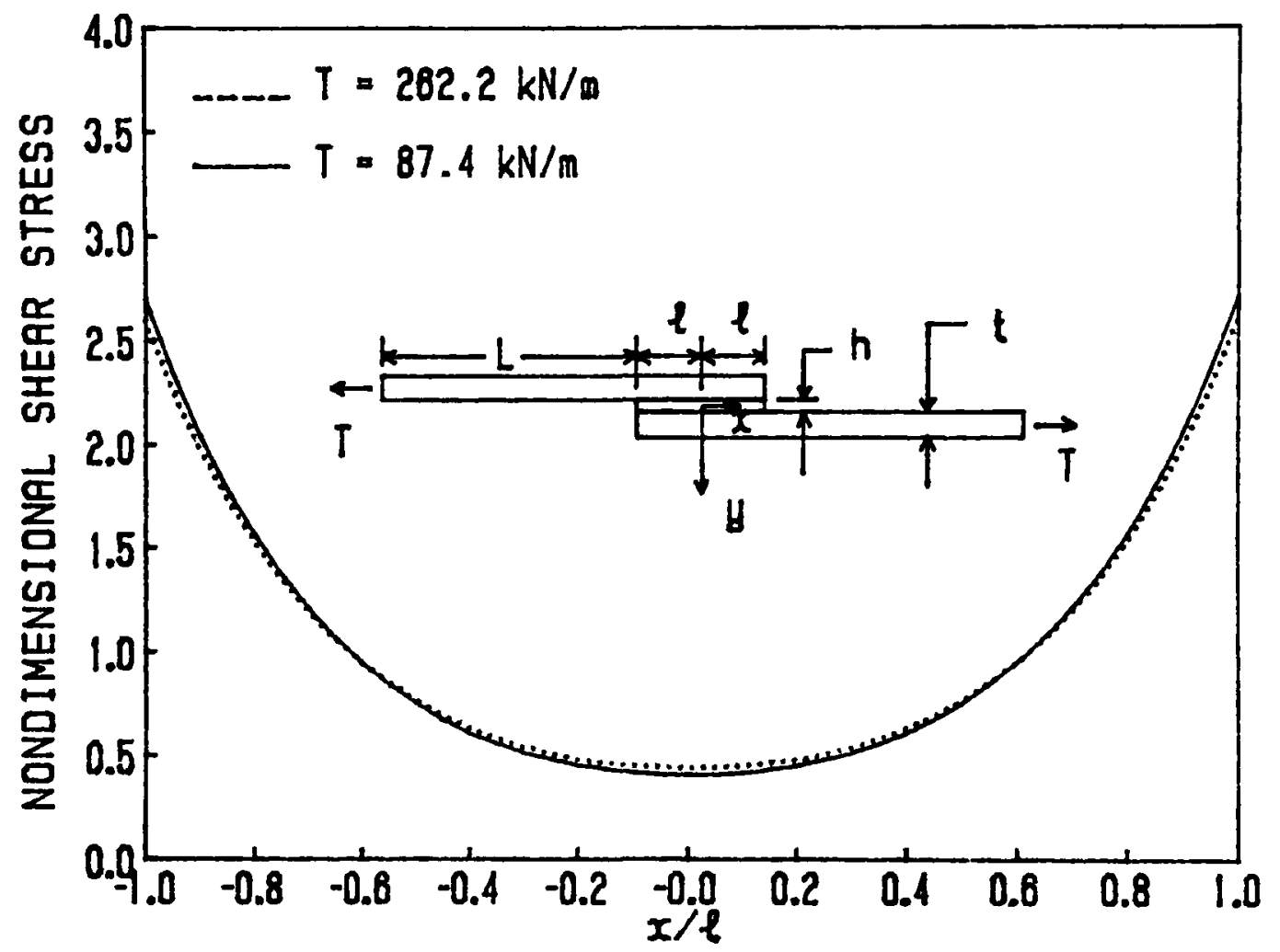

Figure 4.3 : Adhesive shear stress distribution of a steel lap joint for different loads obtained using Equation A.13 with $\mathrm{L}=76.2 \mathrm{~mm} ; \ell=12.7 \mathrm{~mm}$; $\mathrm{h}=0.178 \mathrm{~mm}$ and $t=2.667 \mathrm{~mm}$ 


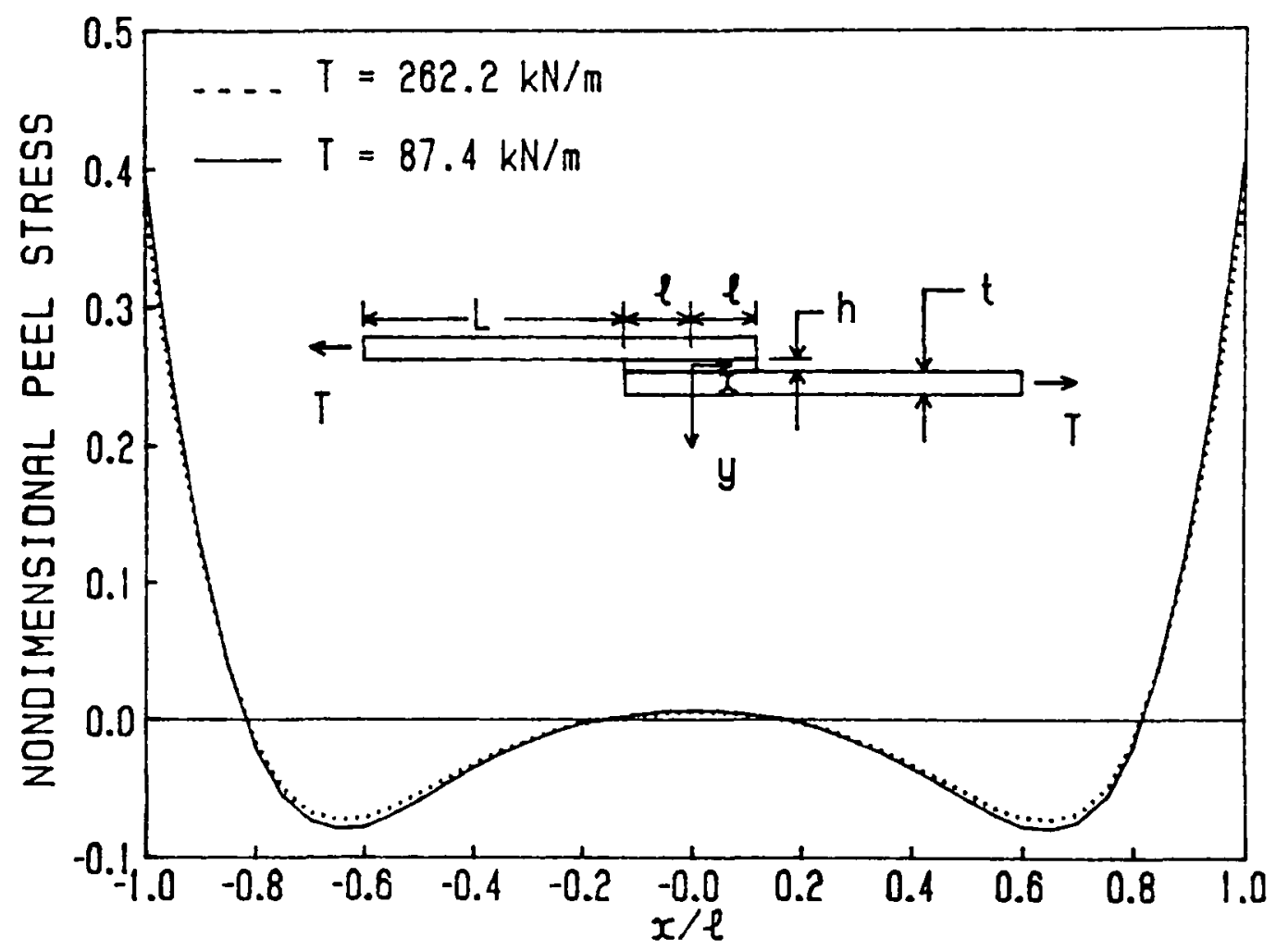

Figure 4.4: Adhesive peel stress distribution of a steel lap joint for different loads obtained using Equation A. 14 with $L=76.2 \mathrm{~mm} ; \quad l=12.7 \mathrm{~mm}$; $h=0.178 \mathrm{~mm}$ and $t=2.667 \mathrm{~mm}$ 


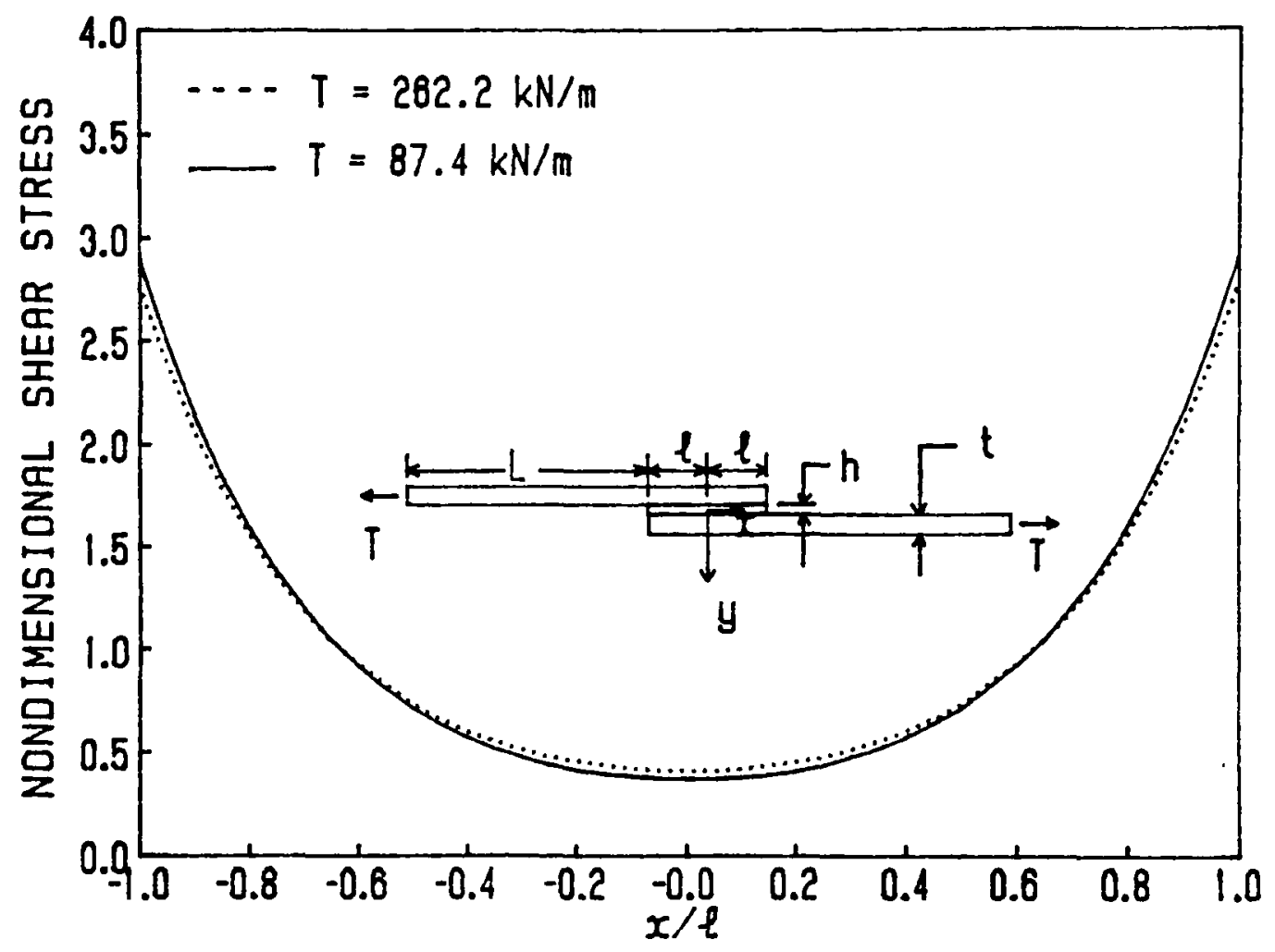

Figure 4.5: Adhesive shear stress distribution of a unidirectional CFRP (composite fibre reinforced plastics) lap joint for different loads obtained using Equation $A .13$ with $L=$ $76.2 \mathrm{~mm} ; l=12.7 \mathrm{~mm} ; \mathrm{h}=0.178 \mathrm{~mm}$ and $t=$ $2.667 \mathrm{~mm}$ 


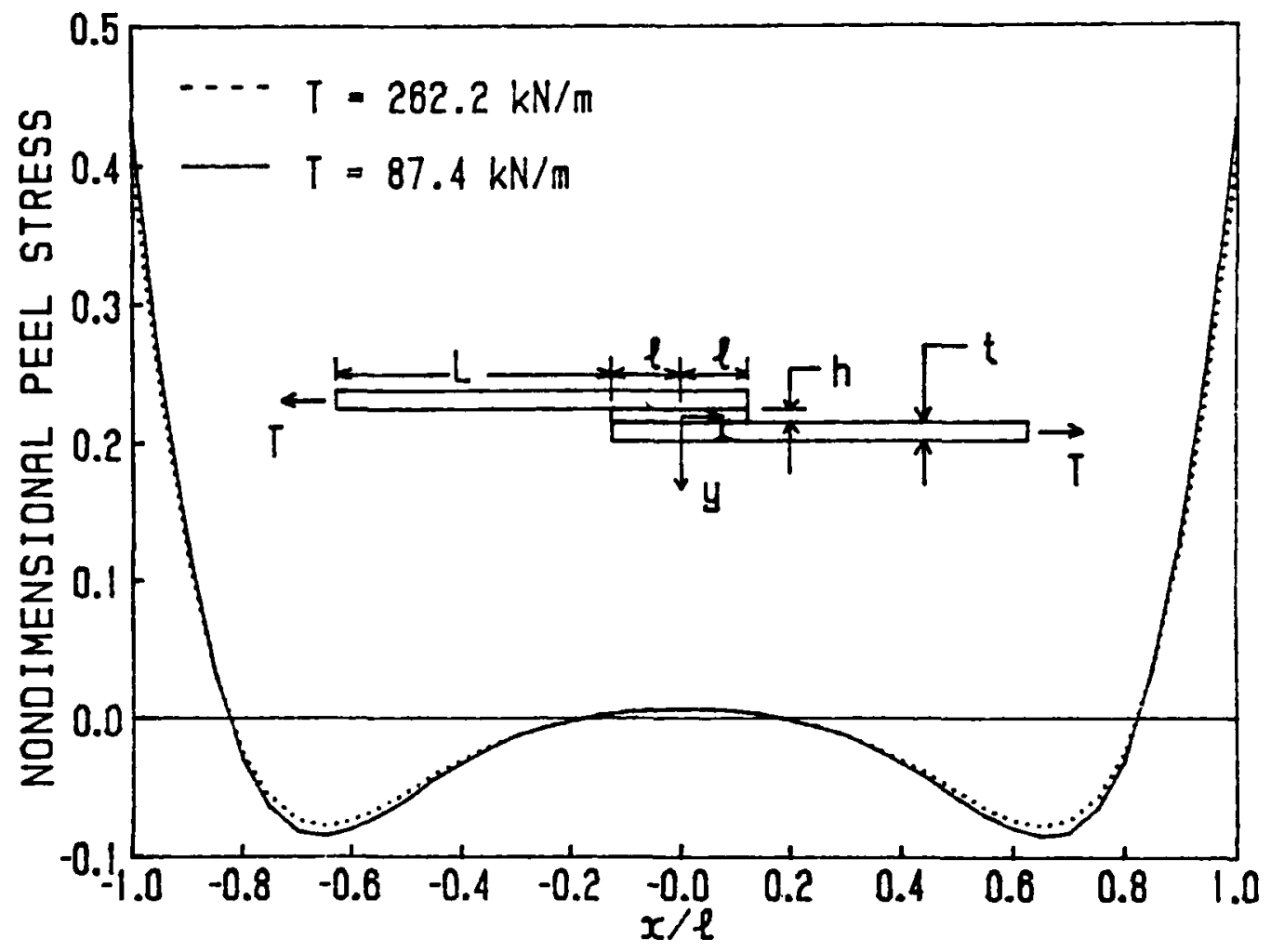

Figure 4.6: Adhesive peel stress distribution of a unidirectional CFRP lap joint for different loads obtained using Equation A.14 with $L=$ $76.2 \mathrm{~mm} ; l=12.7 \mathrm{~mm} ; \mathrm{h}=0.178 \mathrm{~mm}$ and $t=$ $2.667 \mathrm{~mm}$ 


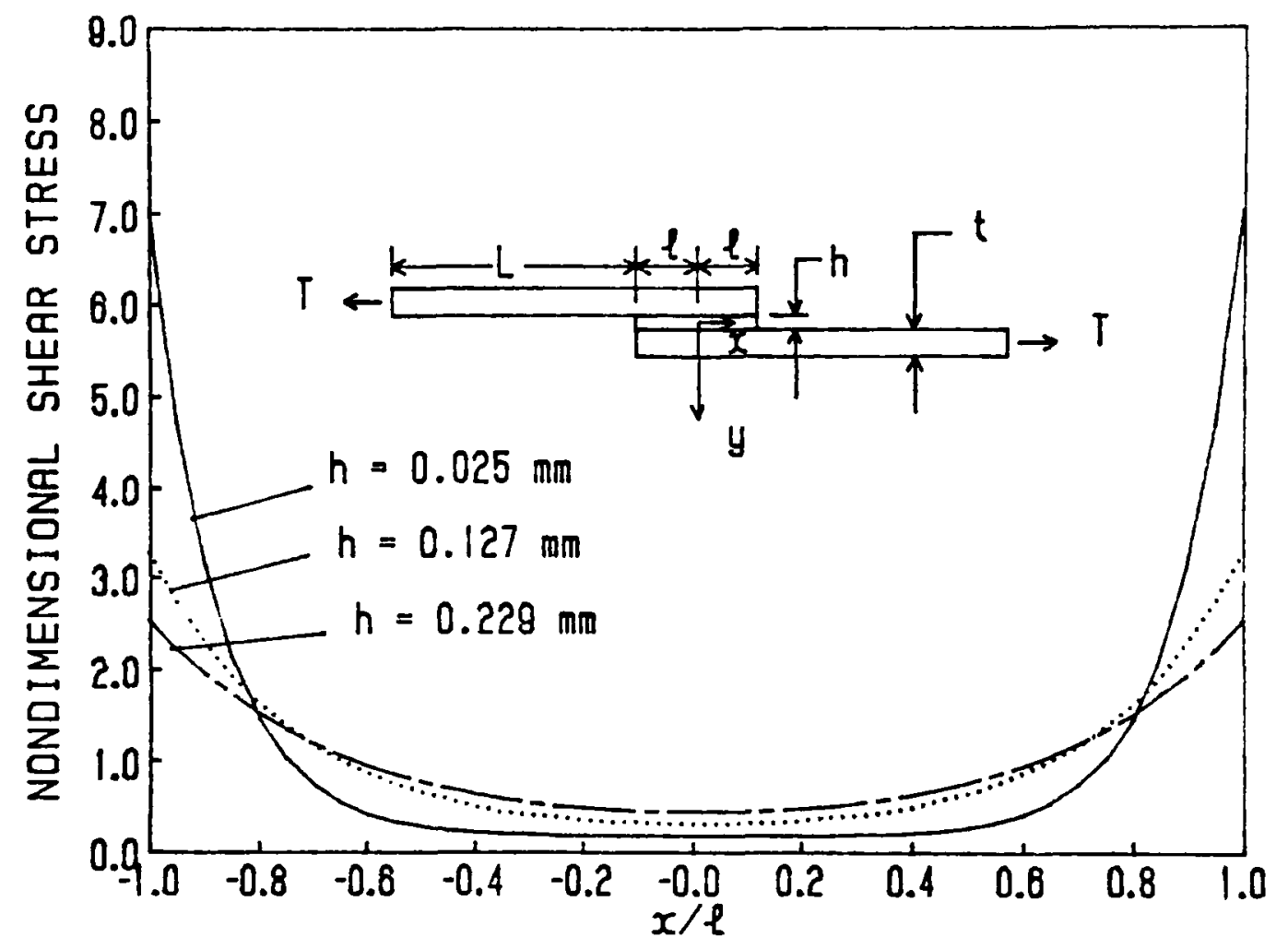

Figure 4.7 : Adhesive shear stress distribution of unidirectional CFRP lap joints for various adhesive thicknesses obtained using Equation A. 13 with $\mathrm{L}=76.2 \mathrm{~mm} ; l=12.7 \mathrm{~mm} ; \mathrm{T}=174.8$ $\mathrm{kN} / \mathrm{m}$ and $t=2.667 \mathrm{~mm}$ 


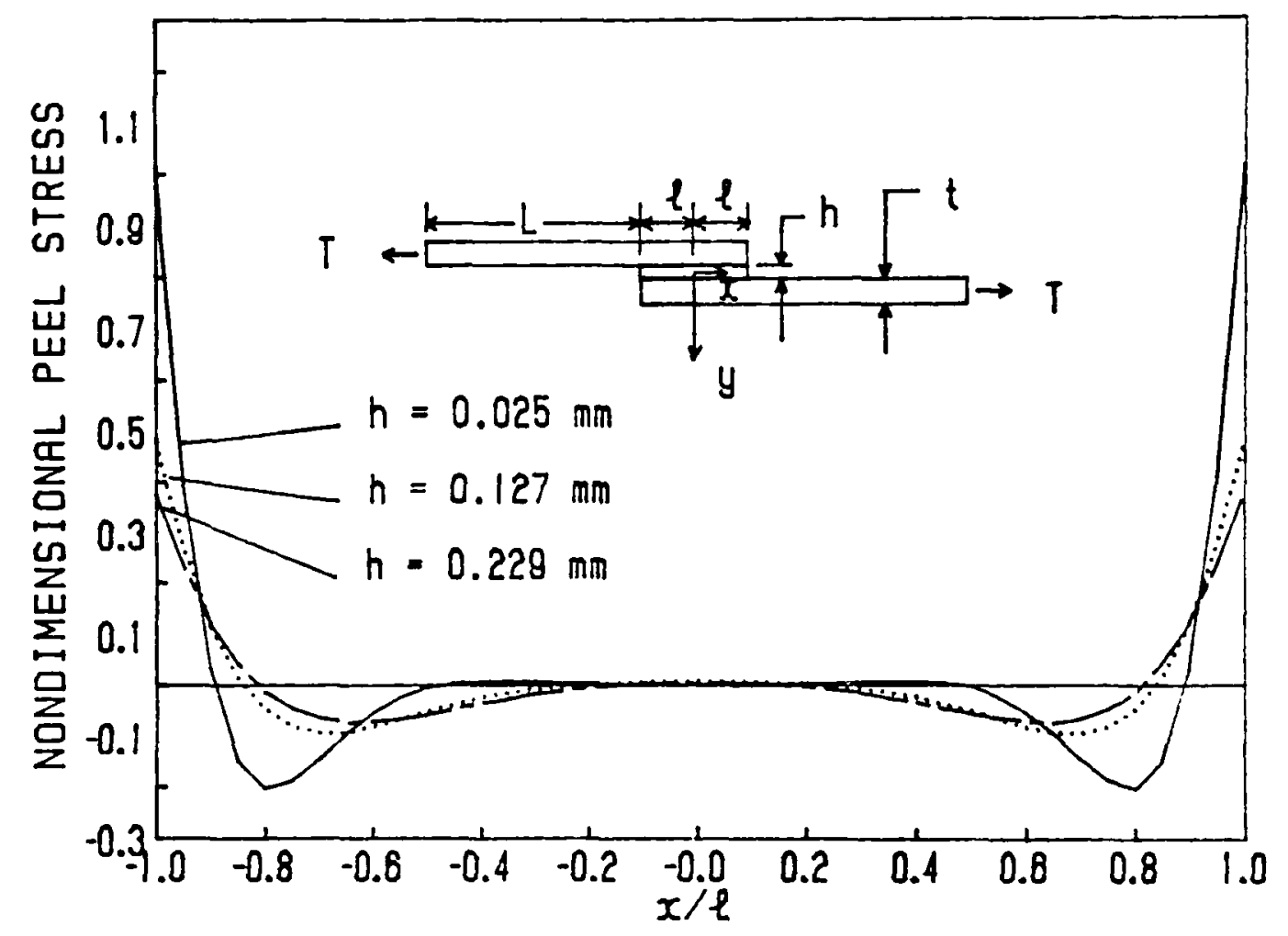

Figure 4.8: Adhesive peel stress distribution of unidirectional CFRP lap joints for various adhesive thicknesses obtained using Equation A.14 with $L=76.2 \mathrm{~mm} ; \ell=12.7 \mathrm{~mm} ; T=174.8 \mathrm{kN} / \mathrm{m}$ and $t=2.667 \mathrm{~mm}$ 


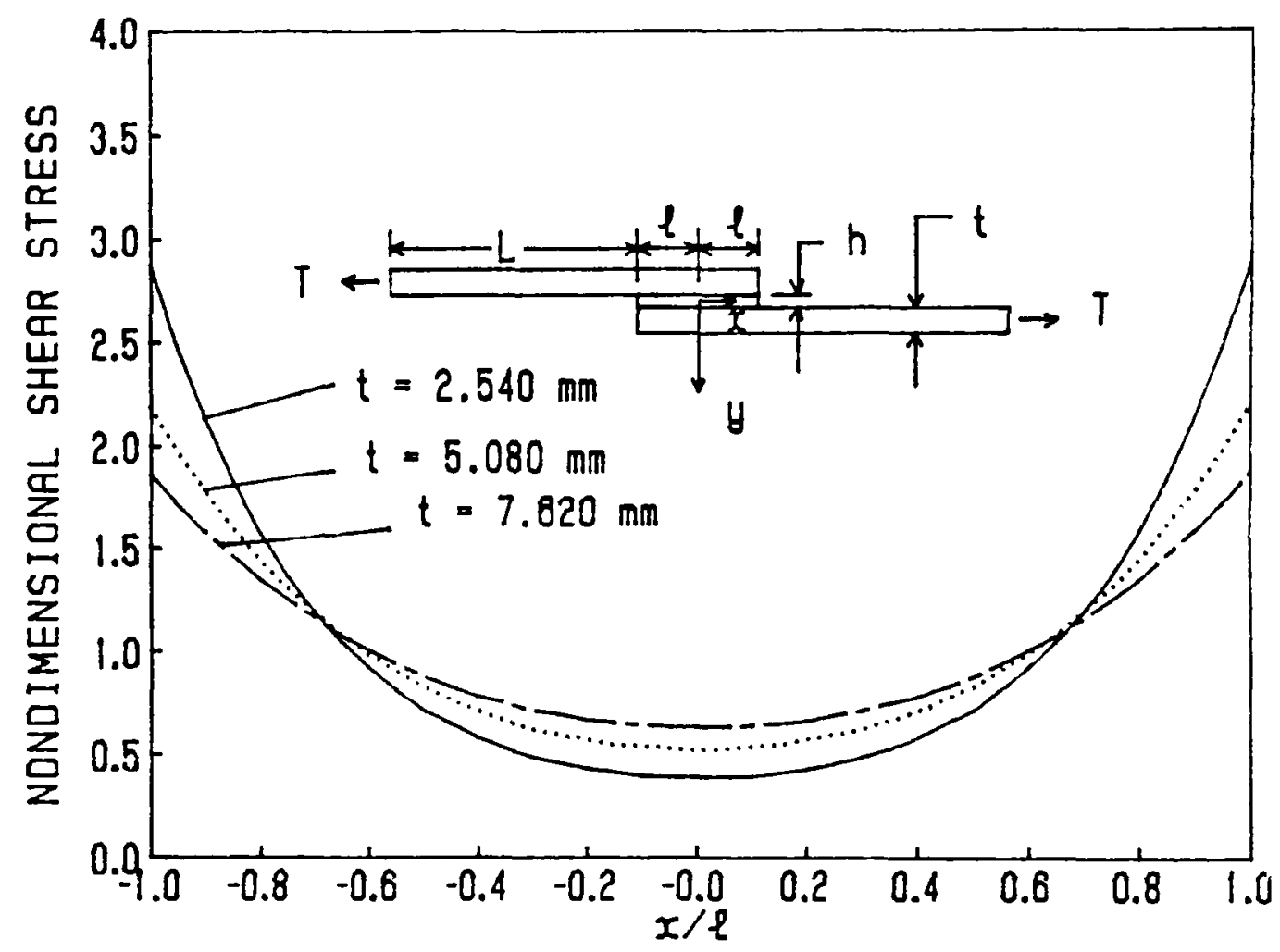

Figure 4.9: Adhesive shear stress distribution of unidirectional CFRP lap joints for several adherend thicknesses obtained using Equation A.13 with $\mathrm{L}=76.2 \mathrm{~mm} ; l=12.7 \mathrm{~mm} ; \mathrm{T}=174.8$ $\mathrm{kN} / \mathrm{m}$ and $\mathrm{h}=0.178 \mathrm{~mm}$ 


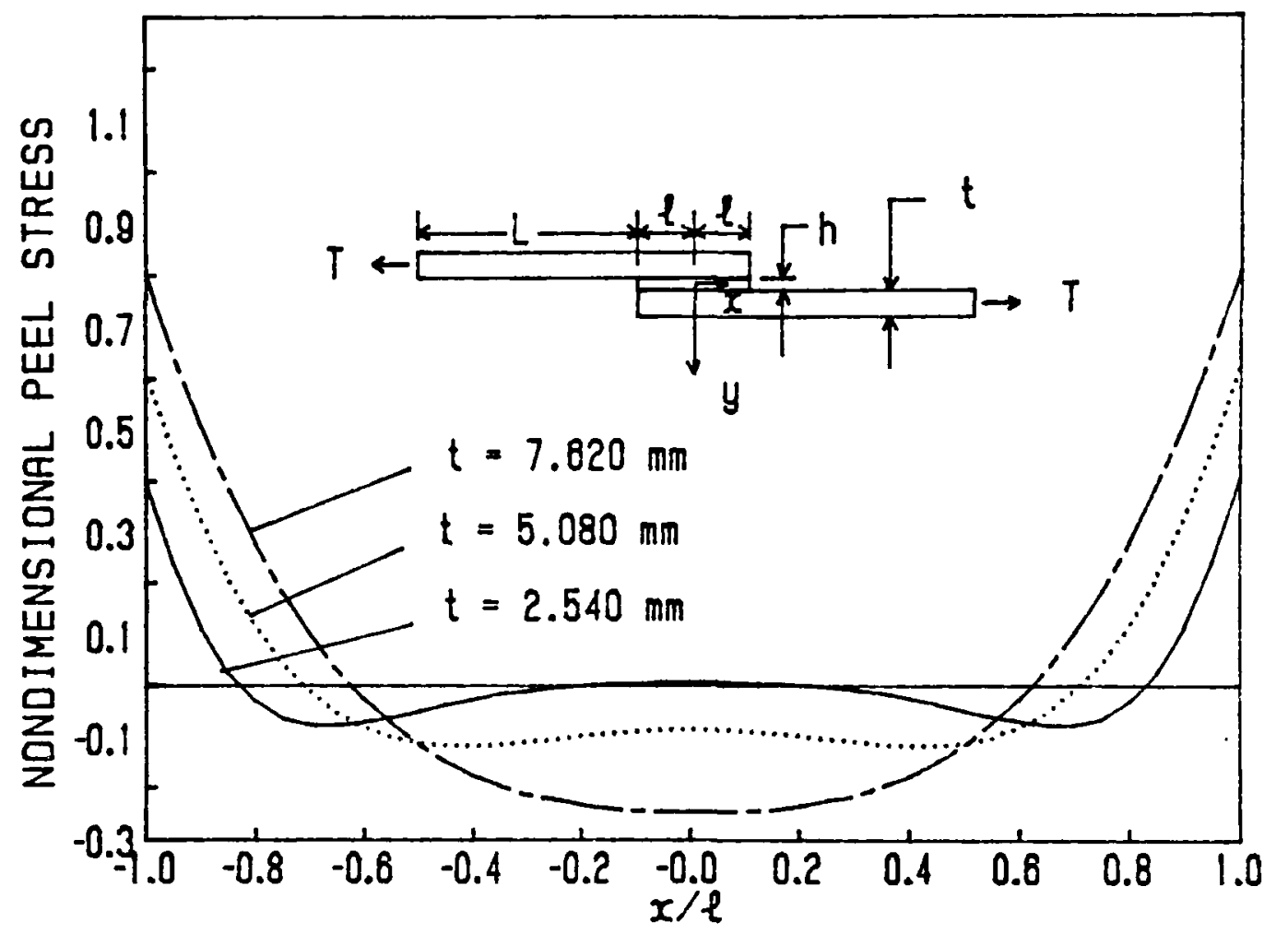

Figure 4.10: Adhesive peel stress distribution of unidirectional CFRP lap joints for several adherend thicknesses obtained using Equation A.14 with $\mathrm{L}=76.2 \mathrm{~mm} ; \ell=12.7 \mathrm{~mm} ; \mathrm{T}=174.8$ $\mathrm{kN} / \mathrm{m}$ and $\mathrm{h}=0.178 \mathrm{~mm}$ 


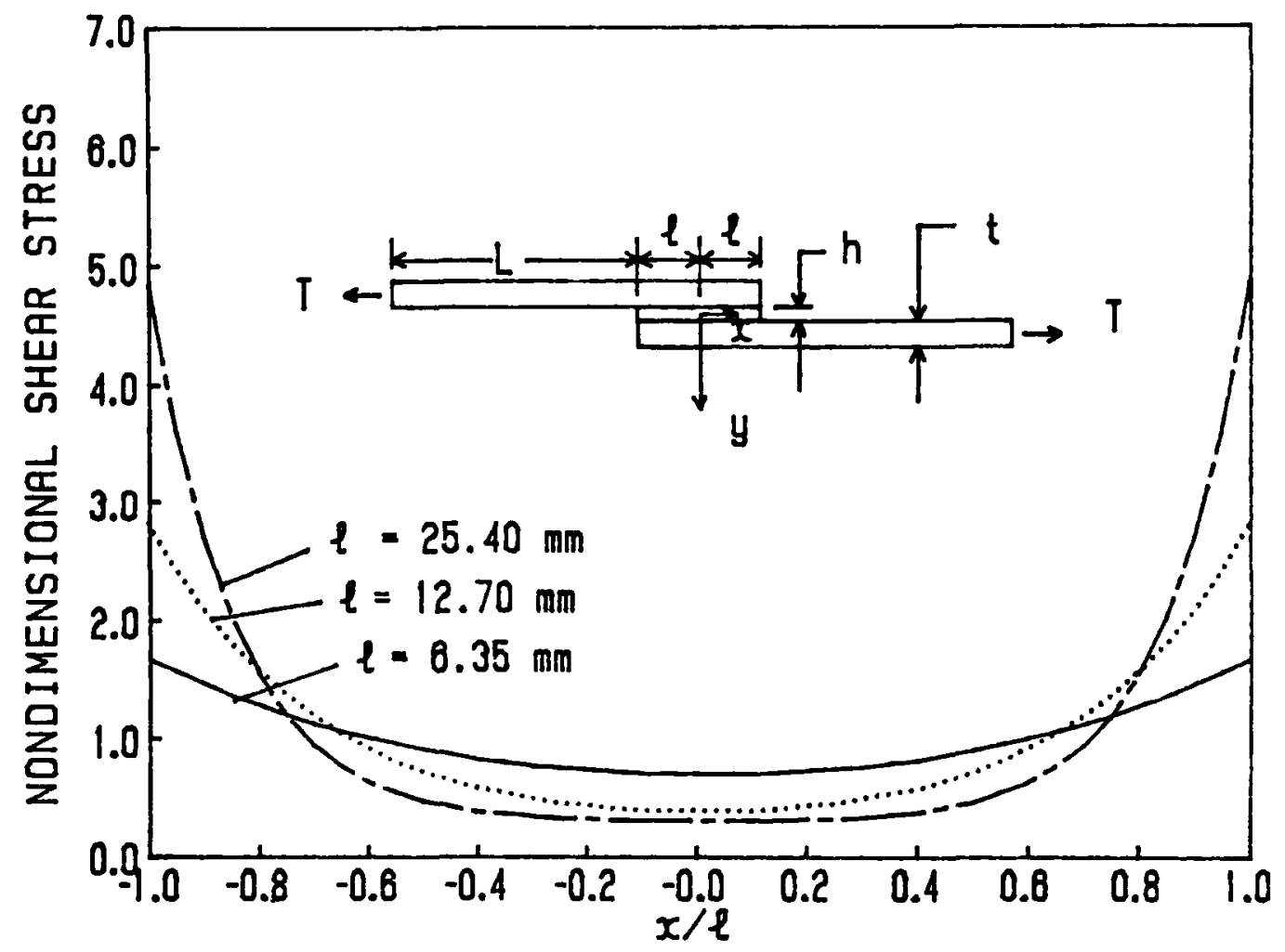

Figure 4.11: Adhesive shear stress distribution of unidirectional CFRP lap joints for various overlap lengths obtained using Equation A.13 with $I=76.2 \mathrm{~mm} ; t=2.667 \mathrm{~mm} ; T=174 \mathrm{kN} / \mathrm{m}$ and $\mathrm{h}=0.178 \mathrm{~mm}$ 


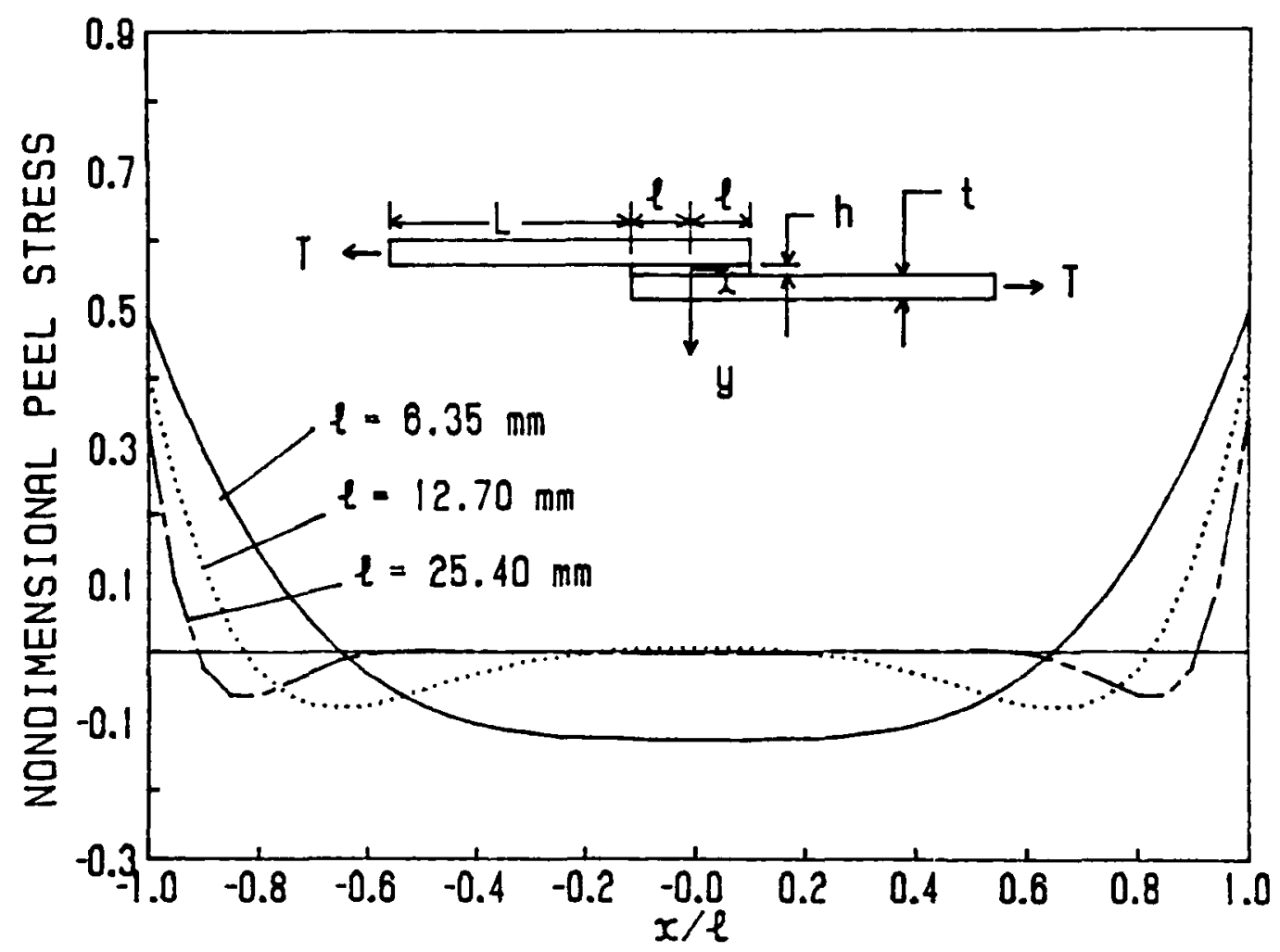

Figure 4.12: Adhesive peel stress distribution of unidirectional CFRP lap joints for various overlap lengths obtained using Equation A.13 with $L=76.2 \mathrm{~mm} ; t=2.667 \mathrm{~mm} ; \mathrm{T}=174.8$ $\mathrm{kN} / \mathrm{m}$ and $\mathrm{h}=0.178 \mathrm{~mm}$ 


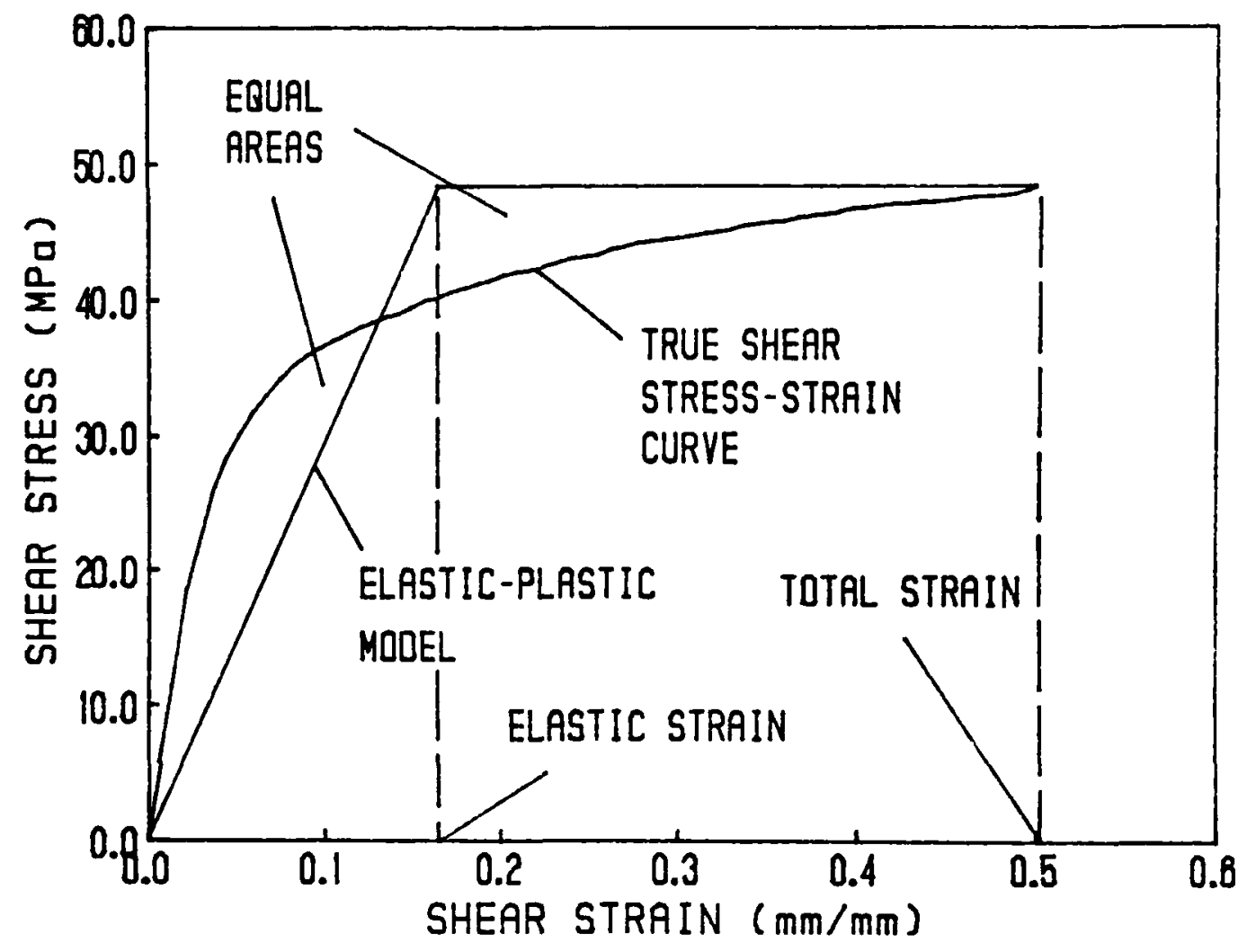

Figure 4.13 : Elastic-plastic model representation of the actual American Cynamid FM300K adhesive shear stress-strain property [16] 


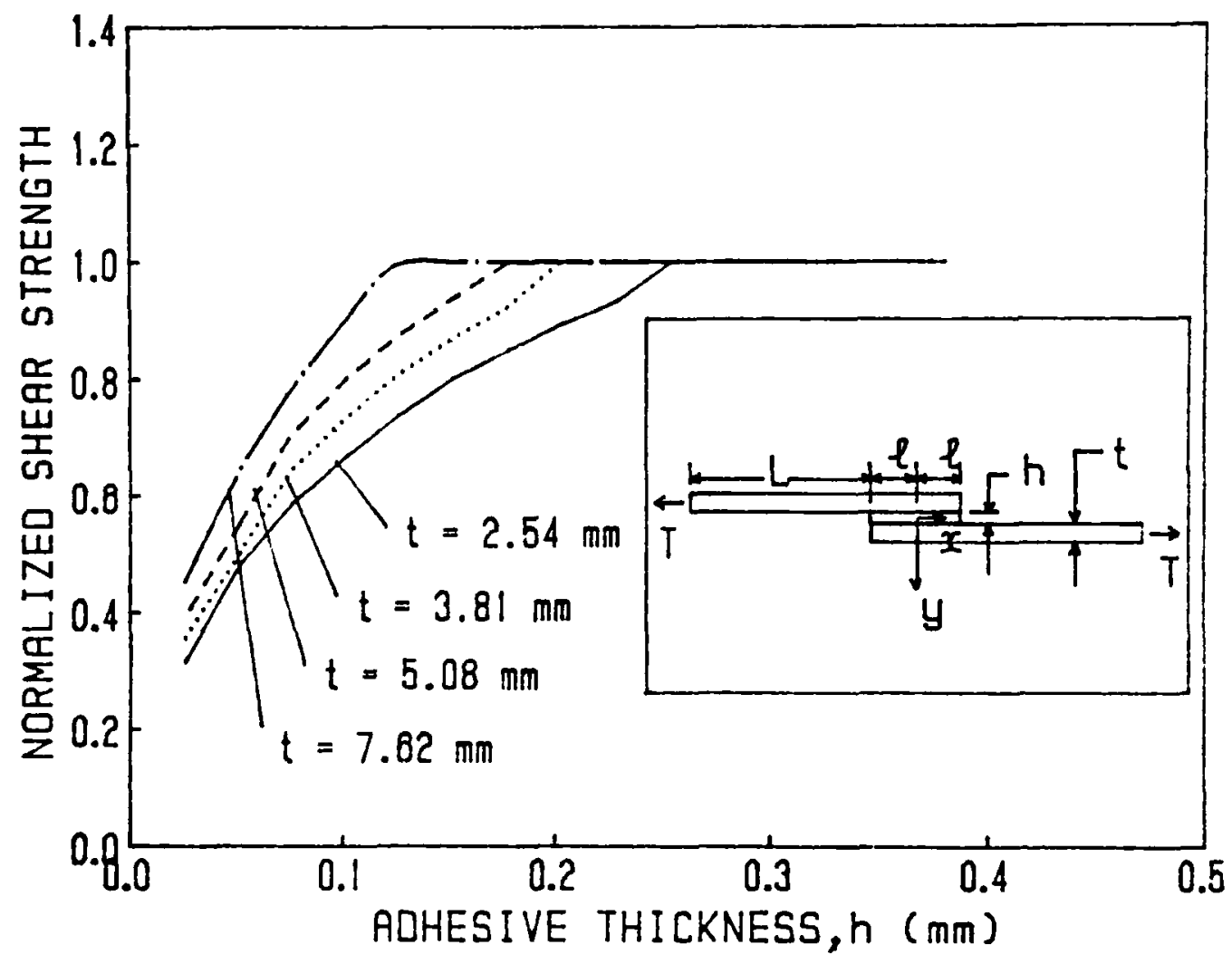

Figure 4.14: Influence of adhesive thickness upon the theoretical shear strength of unidirectional CFRP lap joints for several adherend thicknesses with $L=76.2 \mathrm{~mm}$ and $l=12.70$ $\mathrm{mm}$. The shear strength is normalized with respect to the maximum shear strength of the adhesive, $\tau_{\max }(=48.3 \mathrm{MPa})$ 


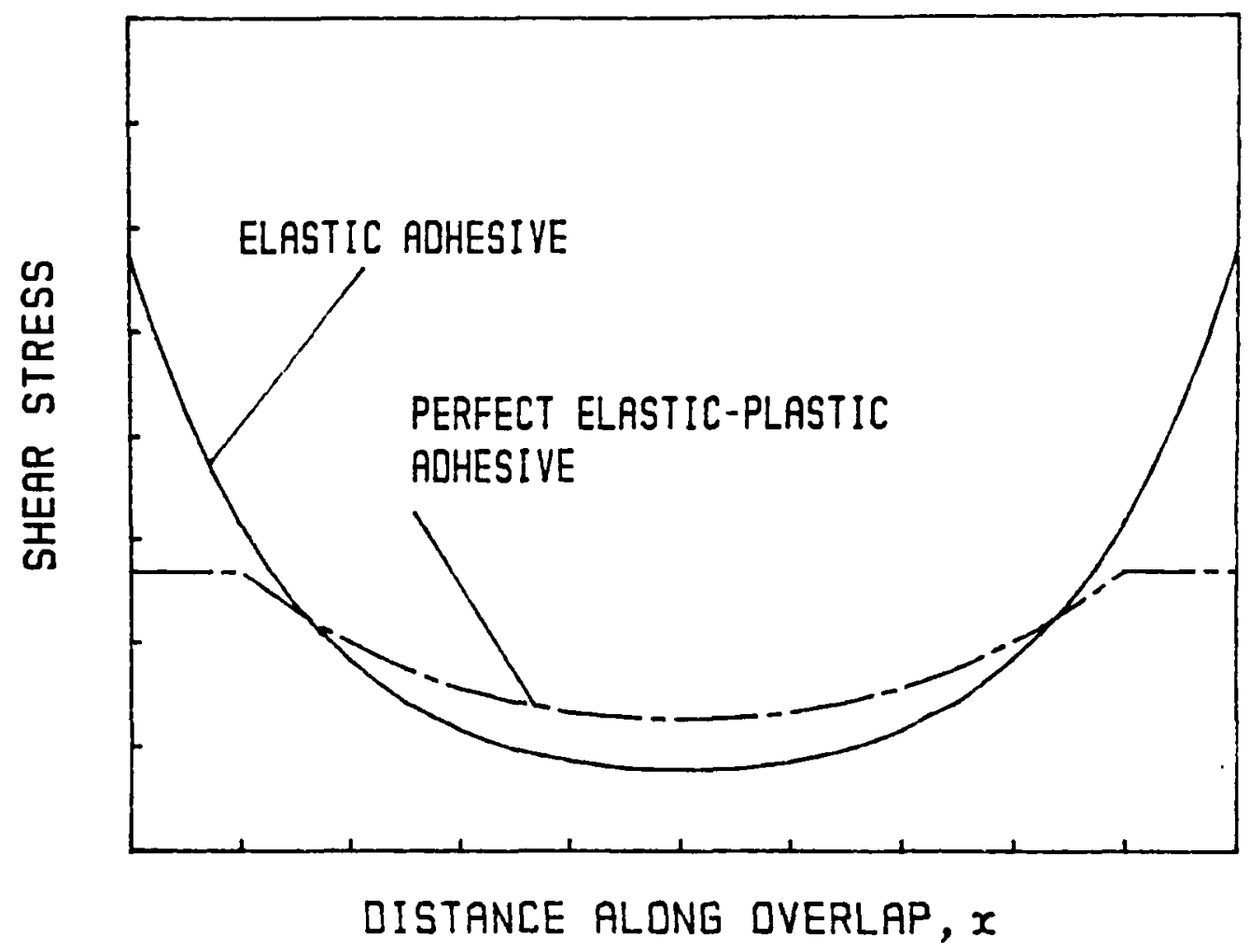

Figure 4.15 : Schematic presentation of the effect of elastic-plastic, as compared with purely elastic behaviour of the adhesive on the stress distribution in a single lap joint 


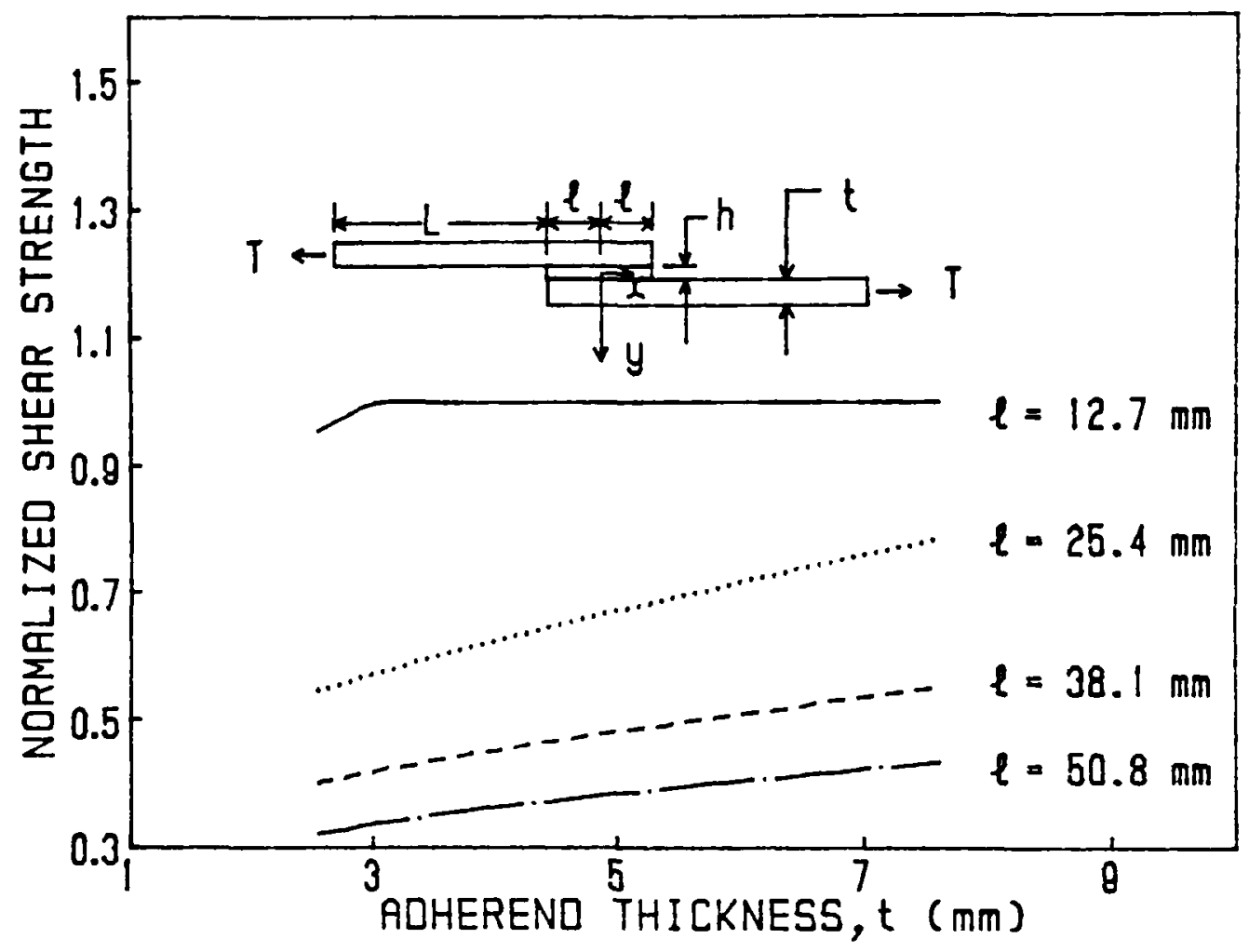

Figure 4.16 : Effect of adhesive thickness on the theoretical shear strength of unidirectional CFRP lap joints for several overlap lengths with $L=76.2 \mathrm{~mm} ; \mathrm{h}=0.076 \mathrm{~mm}$. The shear strength is normalized with respect to the maximum shear strength of the adhesive, $\tau_{\max }$ $(=48.3 \mathrm{Mpa})$ 


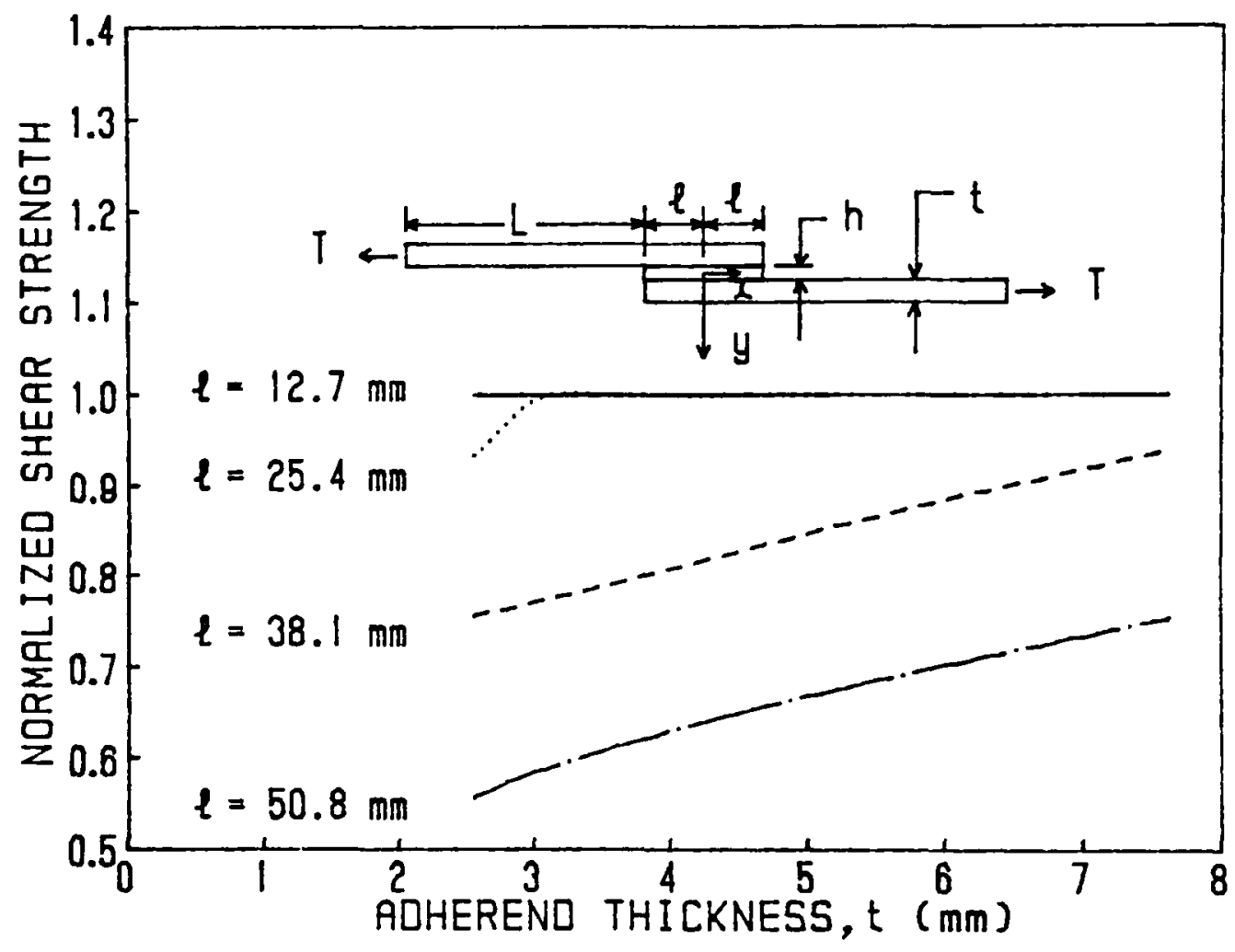

Figure 4.17: Effect of adhesive thickness on the theoretical shear strength of unidirectional CFRP lap joints for several overlap lengths with $\mathrm{L}=76.2 \mathrm{~mm} ; \mathrm{h}=0.229 \mathrm{~mm}$. The shear strength is normalized with respect to the maximum shear strength of the adhesive, $\tau_{\max }$ $(=48.3 \mathrm{Mpa})$ 


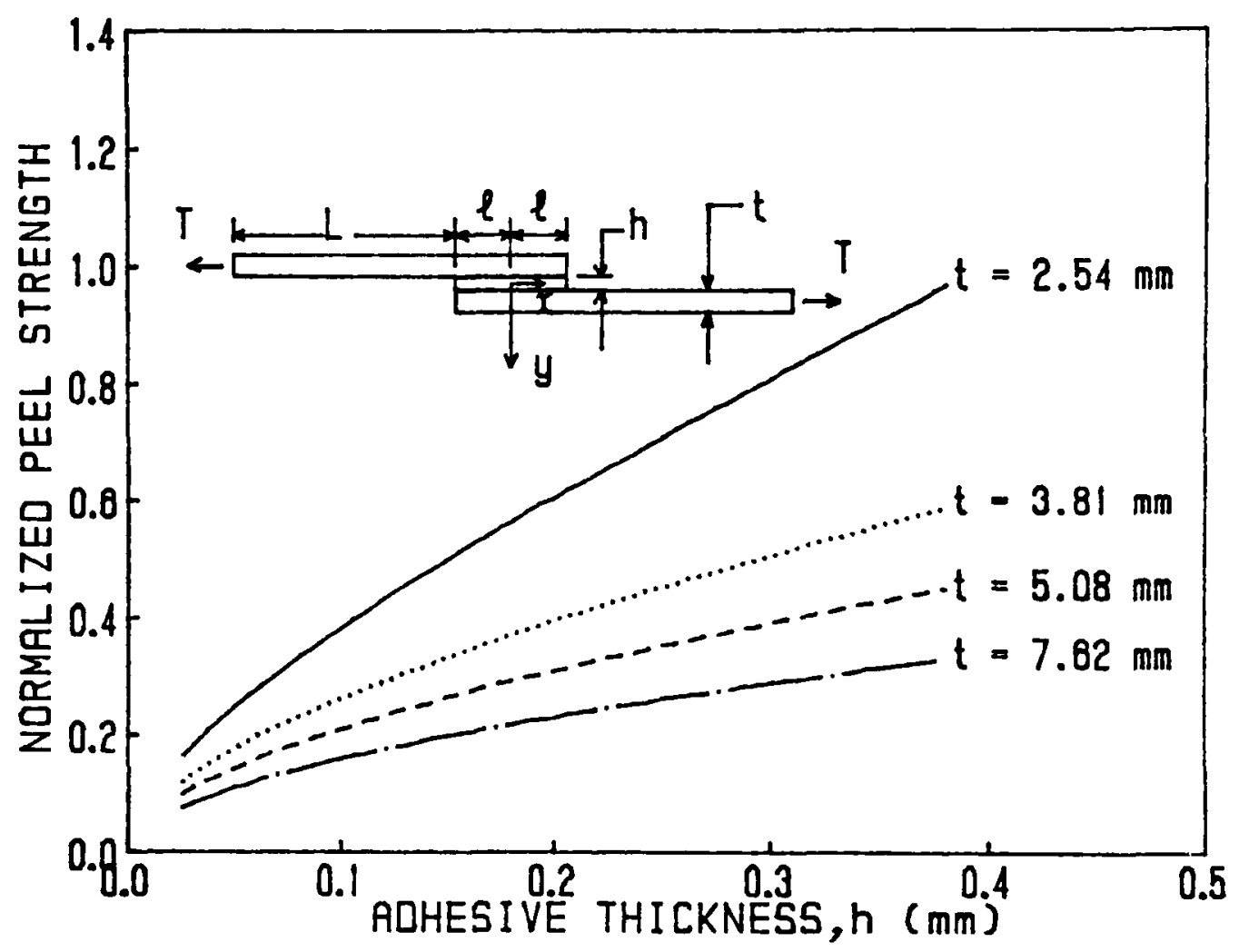

Figure 4.18 : Theoretical peel strength of unidirectional CFRP lap joints as a function of adhesive thickness for various adherend thickness with $\mathrm{L}=76.2 \mathrm{~mm}$ and $l=12.7 \mathrm{~mm}$. The peel strength is normalized with respect to the maximum peel strength (interlaminar strength) of the composite adherend, $\sigma_{Y(\max )}(=60.0$ $\mathrm{MPa}$ ) 


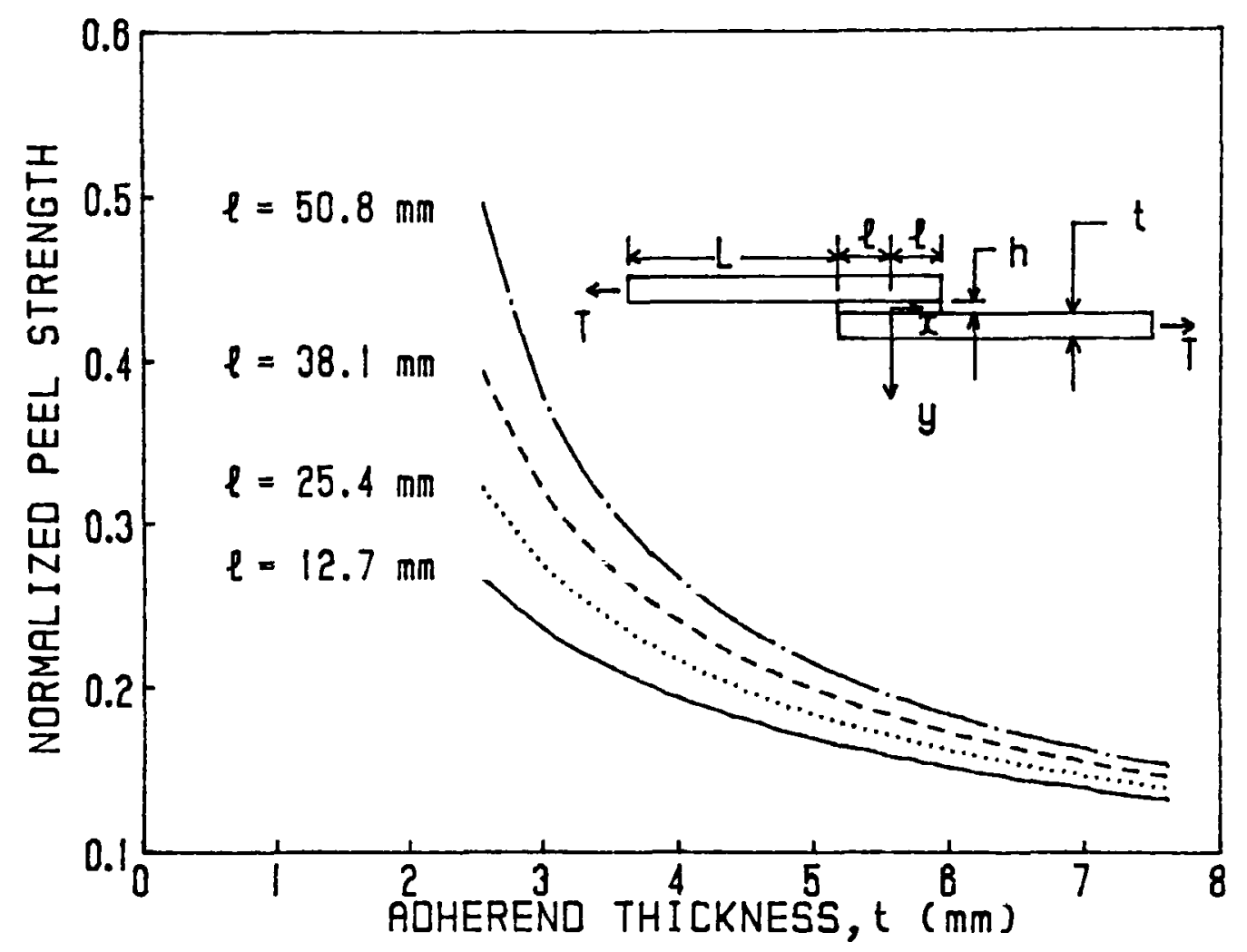

Figure 4.19: Theoretical peel strength of unidirectional CFRP lap joints as a function of adherend thickness for various overlap lengths with $\mathrm{L}=76.2 \mathrm{~mm}$ and $\mathrm{h}=$ $0.076 \mathrm{~mm}$. The peel strength is normalized with respect to the maximum peel strength (interlaminar strength) of the composite adherend, $\sigma_{Y(\max ) \quad(=}$ $60.0 \mathrm{MPa}$ ) 


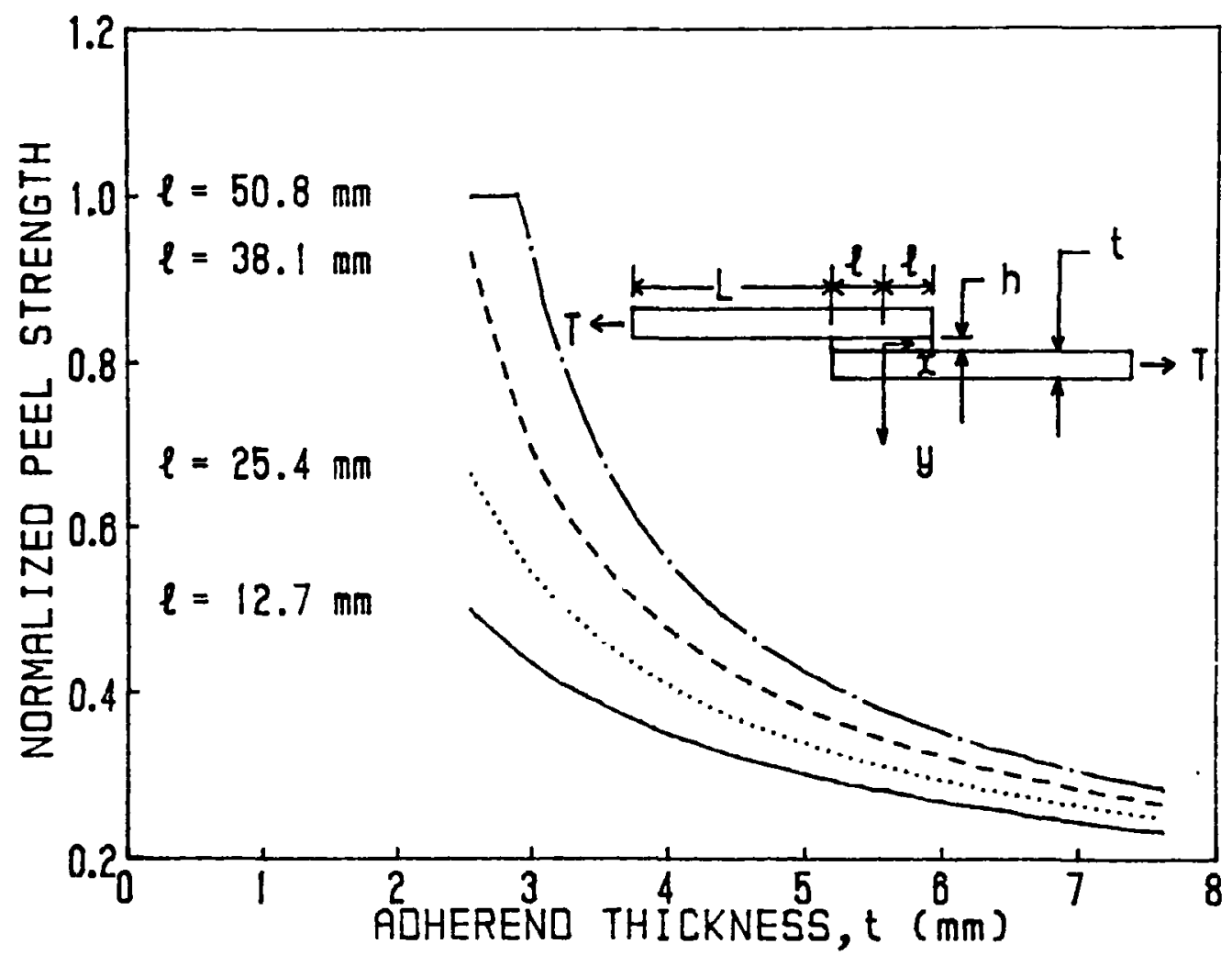

Figure 4.20: Theoretical peel strength of unidirectional CFRP lap joints as a function of adherend thickness for various overlap lengths with $L$ $=76.2 \mathrm{~mm}$ and $\mathrm{h}=0.229 \mathrm{~mm}$. The peel strength is normalized with respect to the maximum peel strength (interlaminar strength) of the composite adherend, $\sigma_{y}(\max )(=60.0 \mathrm{MPa})$ 


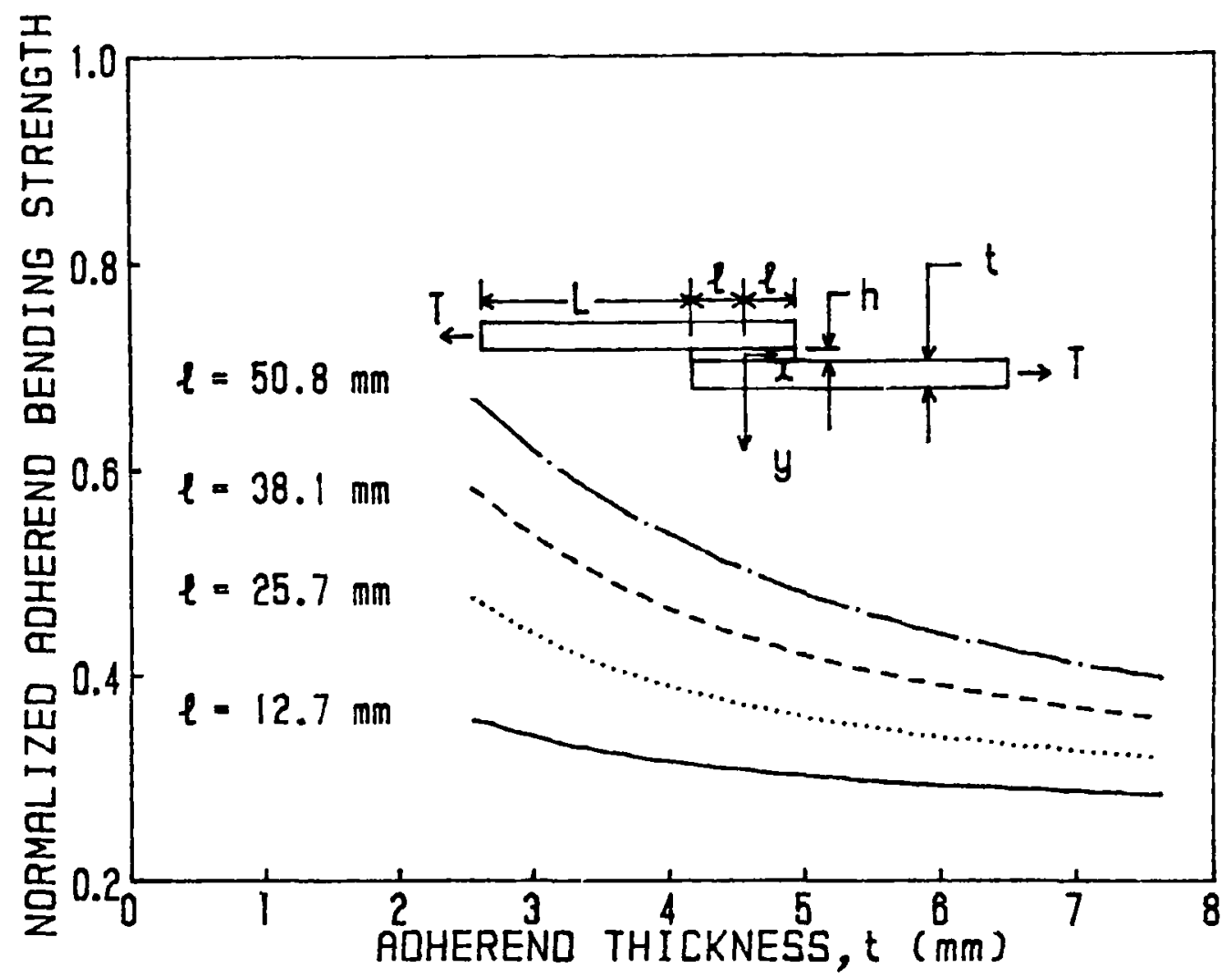

Figure 4.21: Influence of adherend thickness upon the adherend bending strength of unidirectional CFRP lap joints with $\mathrm{L}=76.2 \mathrm{~mm}$ and $\mathrm{h}=$ $0.076 \mathrm{~mm}$. The adherend bending strength is normalized with respect to the maximum tensile strength of the adherend, $\sigma_{\max }(=$ $2.6 \mathrm{GPa})$ 


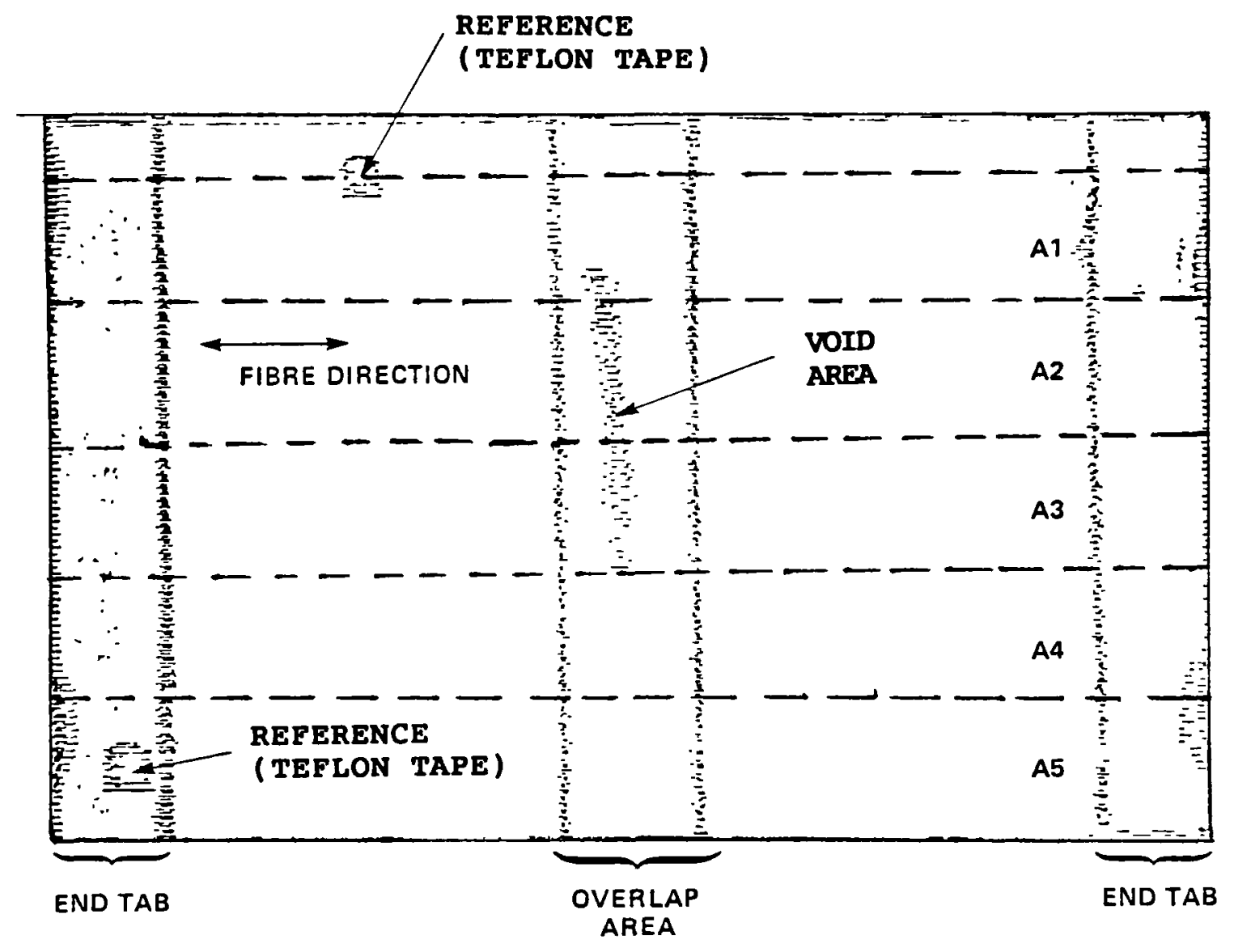

Figure 5.1: Ultrasonic c-scan result of panel no.1 (specimen type A) 


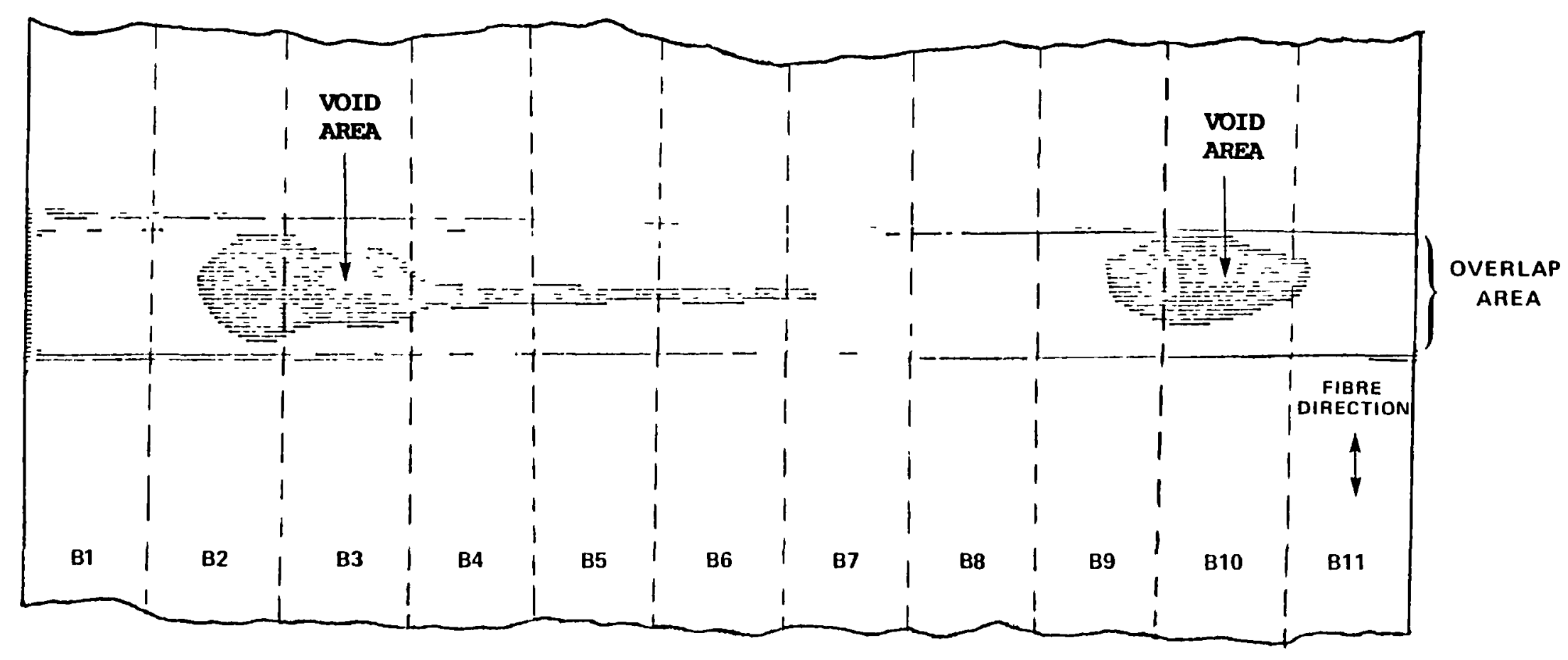

Figure 5.2 : Ultrasonic c-scan result of panel no.2 (specimen type B) 

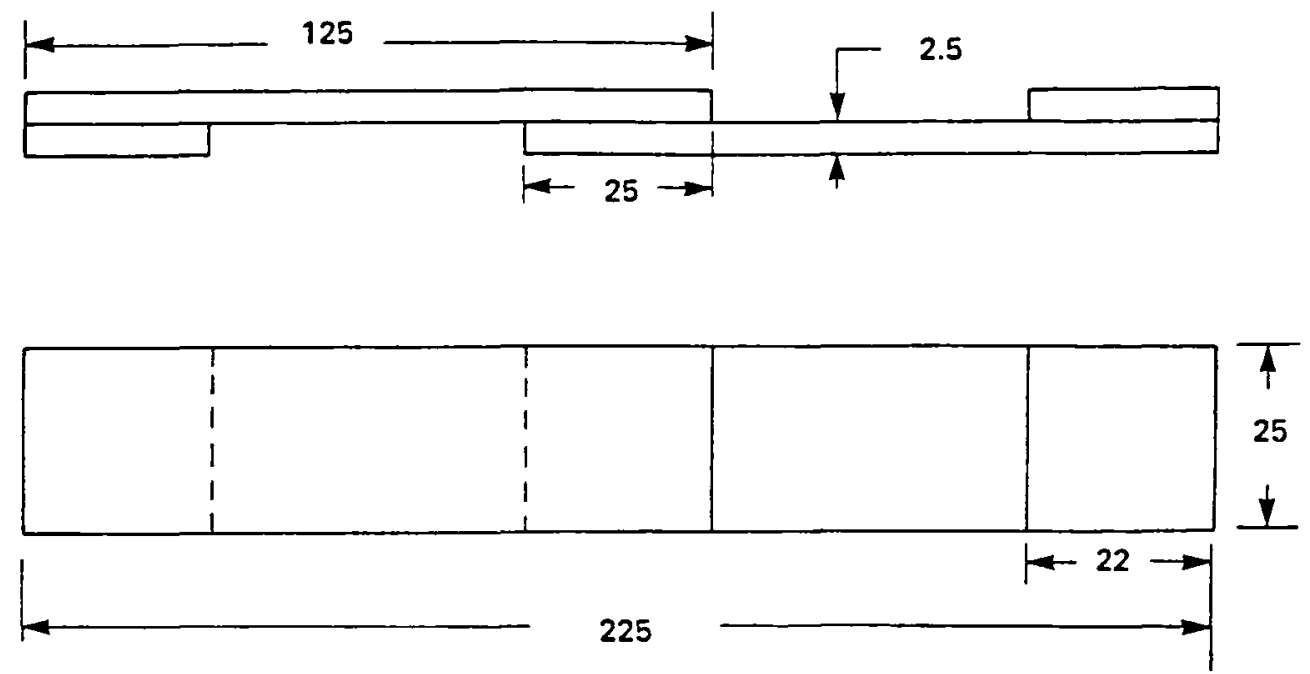

(a) SPECIMEN TYPE A
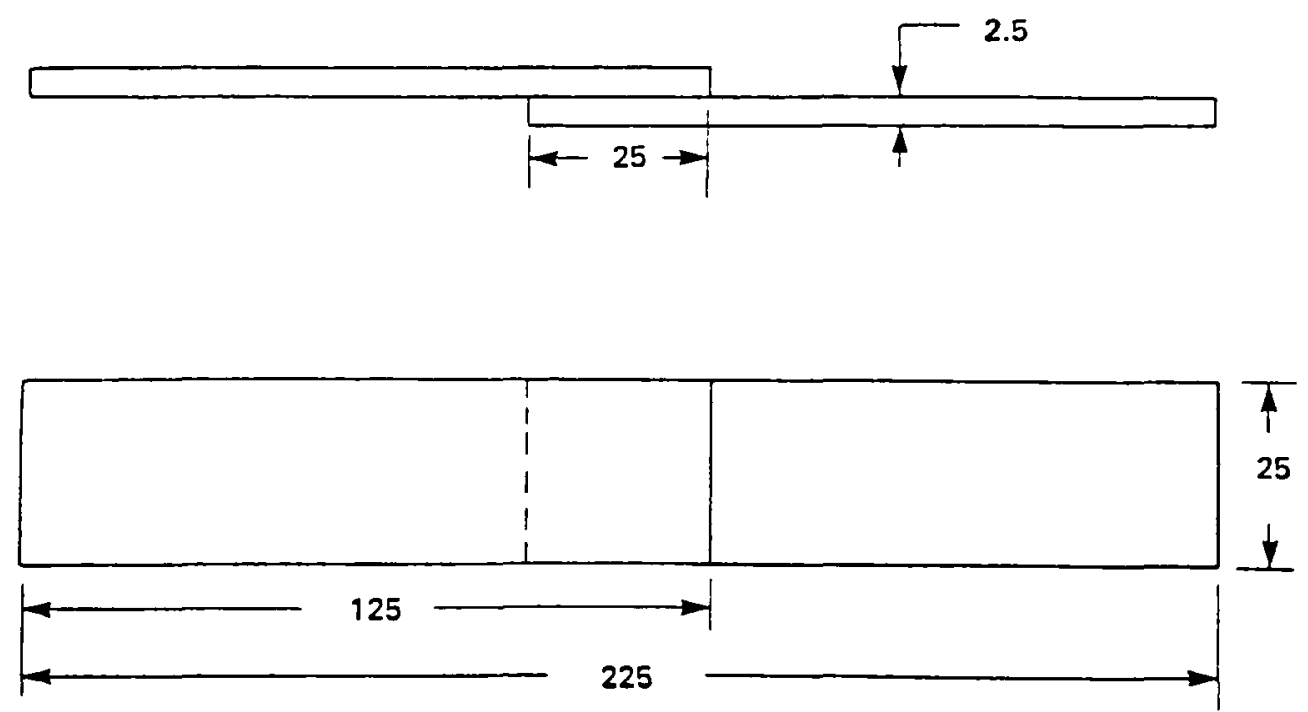

(b) SPECIMEN TYPE B

Figure 5.3 : Test specimen configurations (dimensions are in $\mathrm{mm}$ ) 


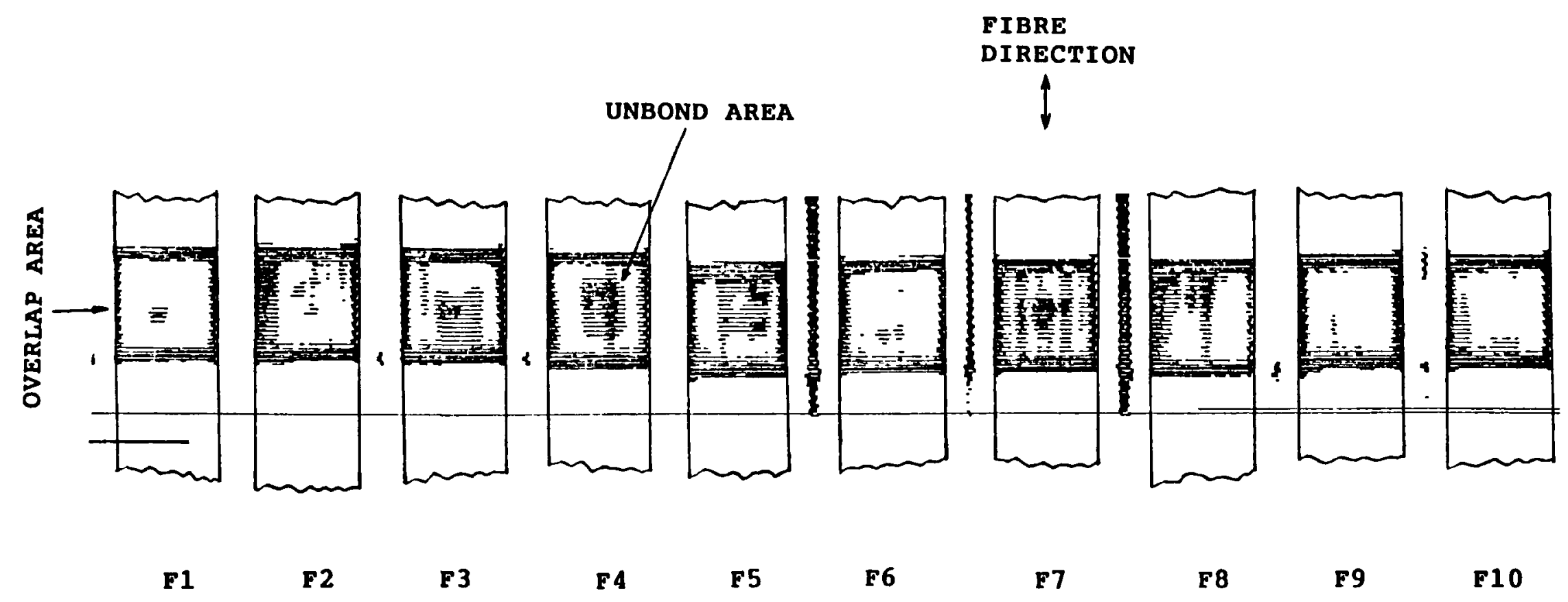

Figure 5.4 : Ultrasonic c-scan result of specimens with unbonds 


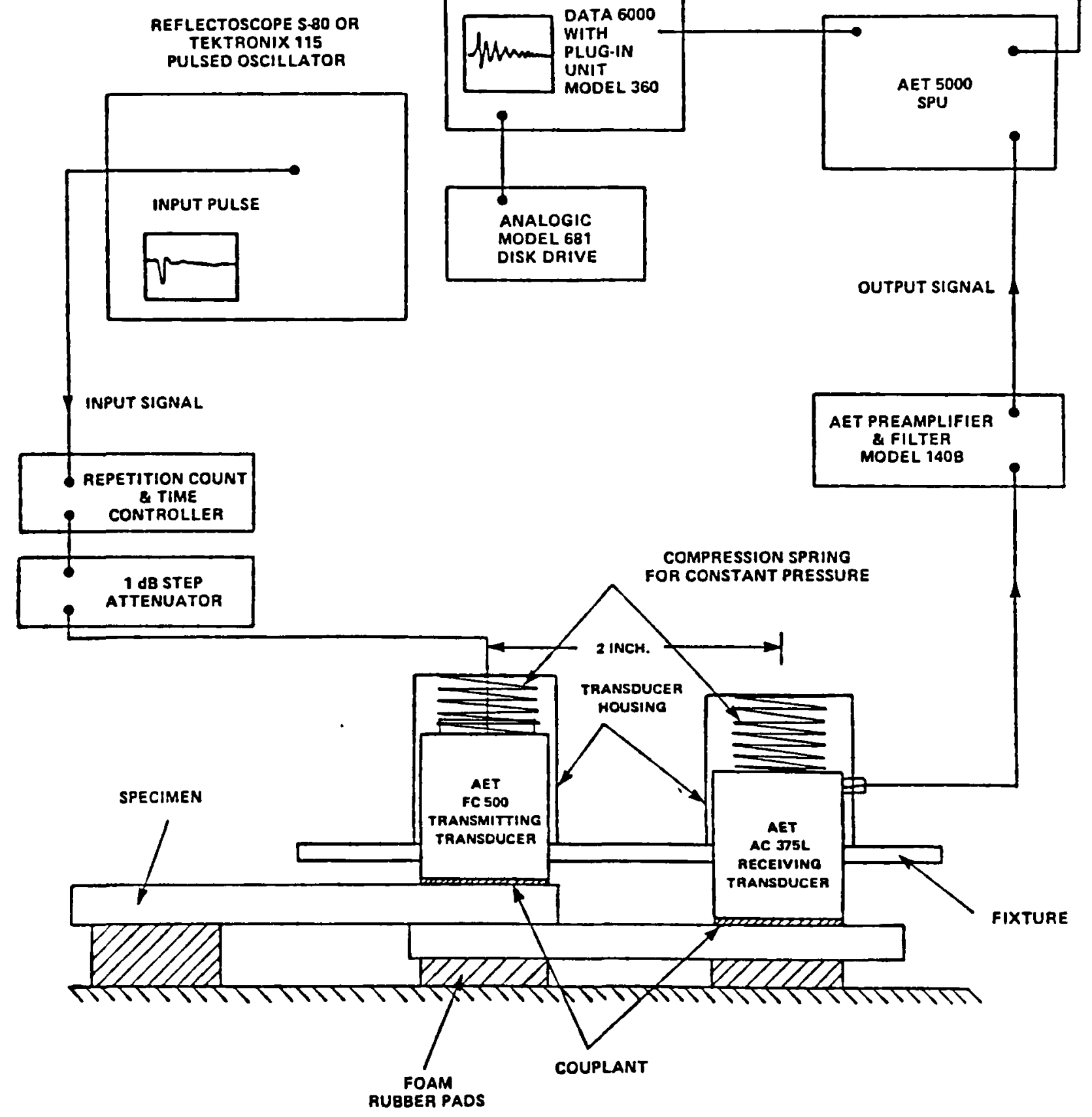

Figure 5.5: A schematic diagram of the acousto-ultrasonic system as used in the present investigation 


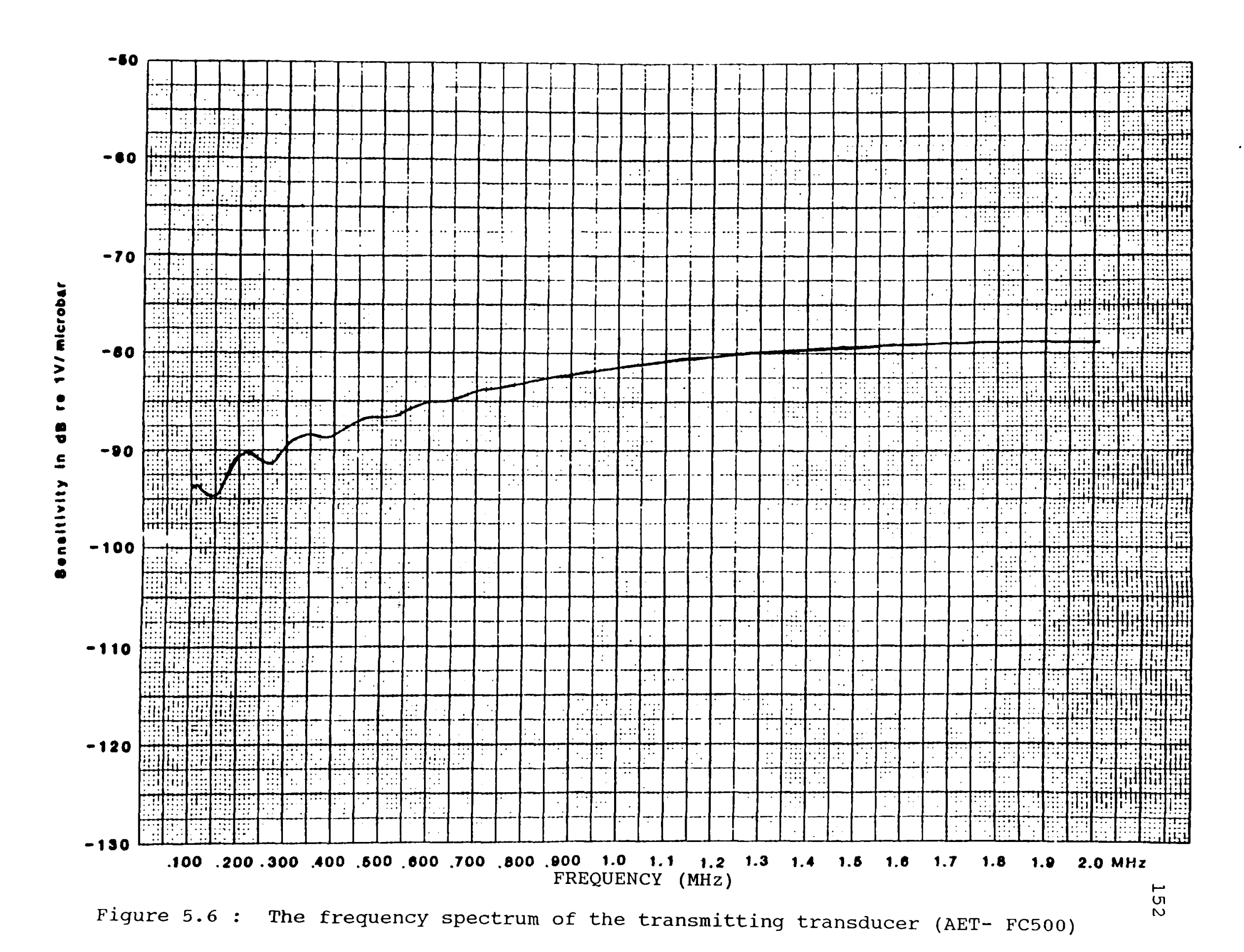




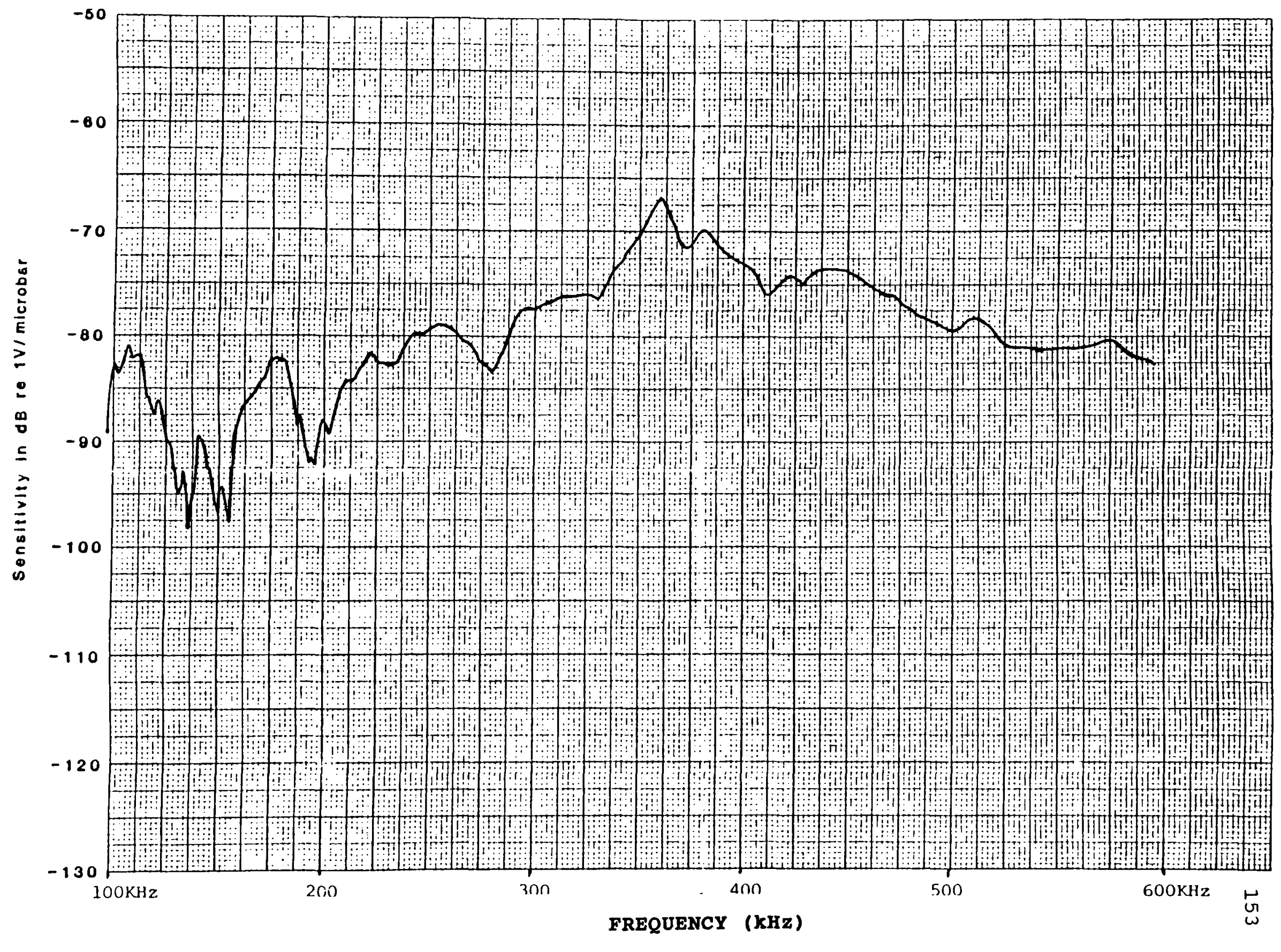

Figure 5.7 : The frequency spectrum of the receiving transducer (AET-AC375) 


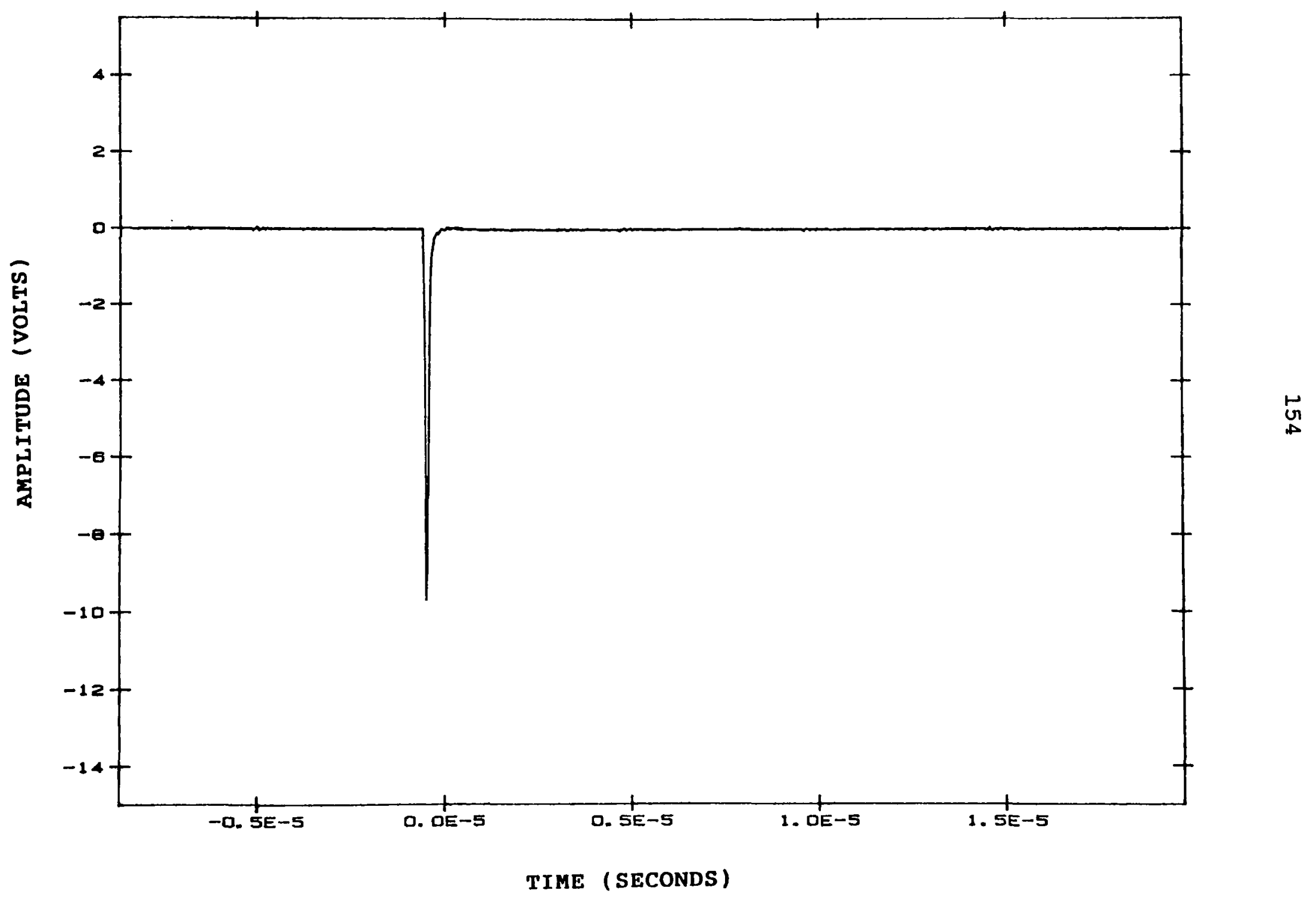

Figure 5.8: Input pulse to the transmitting transducer for AU measurement 


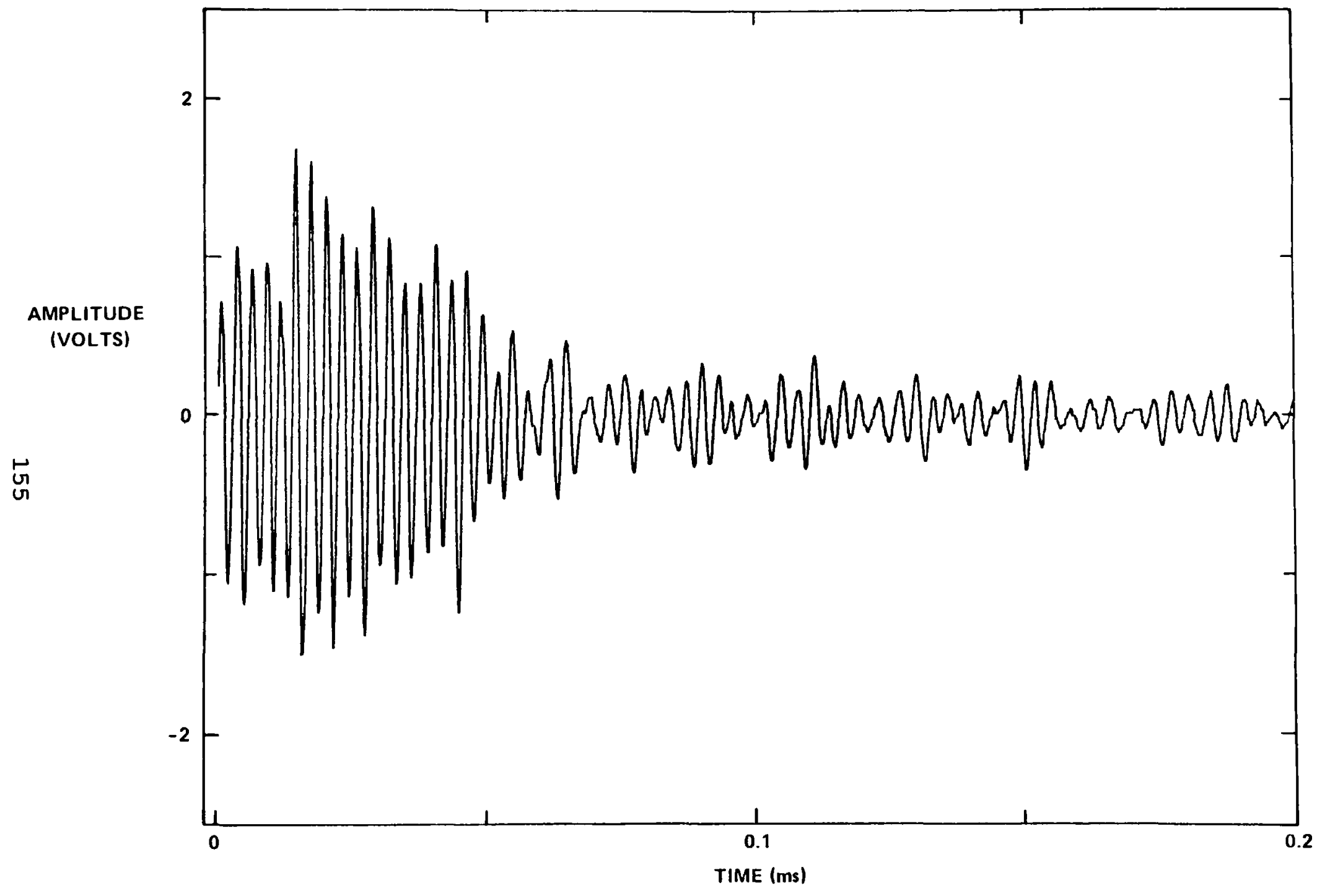
Figure 5.9(a): A typical acousto-ultrasonic waveform for a relatively good 


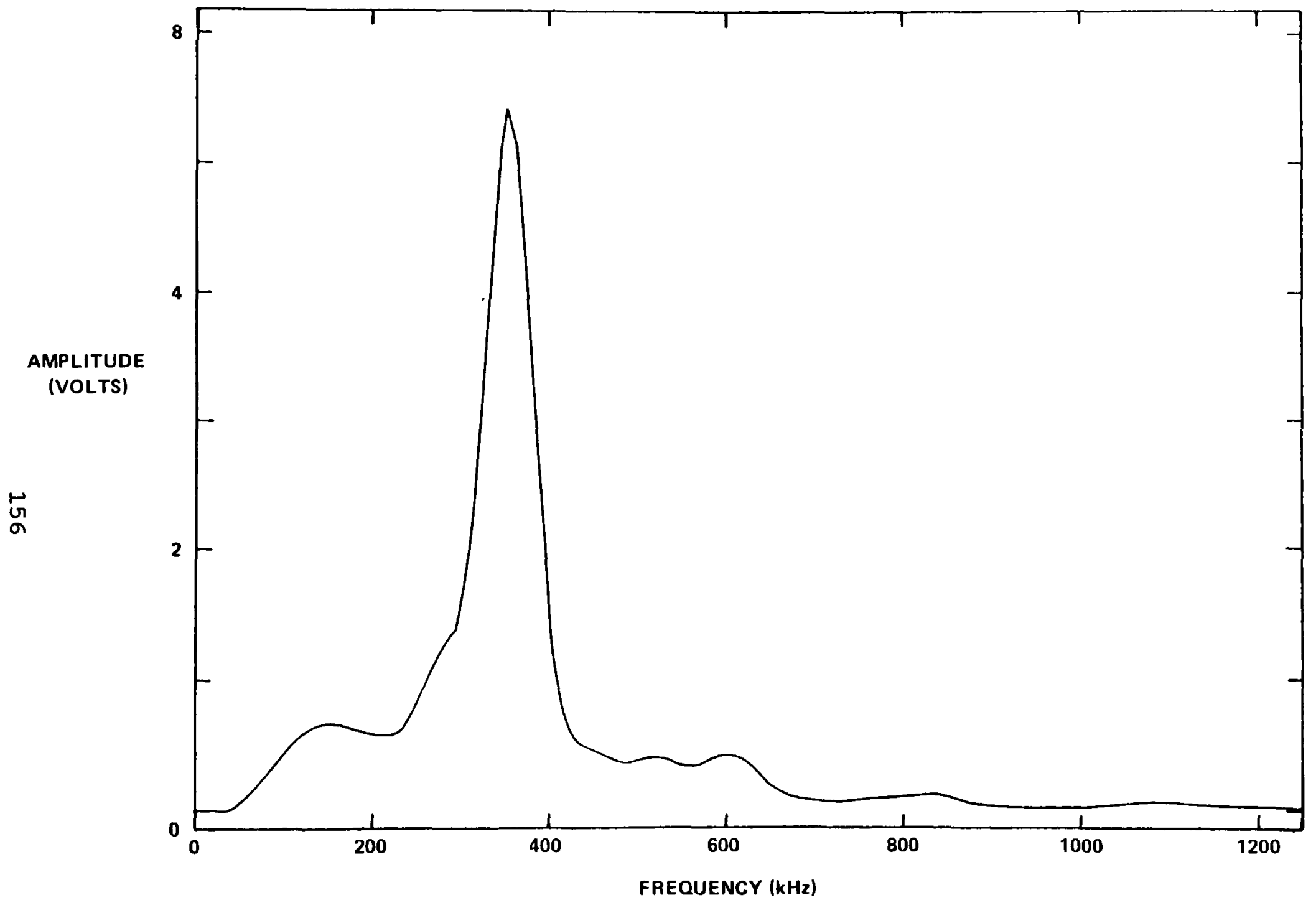

Figure 5.9(b): Frequency spectrum of the acousto-ultrasonic waveform shown in Figure $5.9(\mathrm{a})$ 


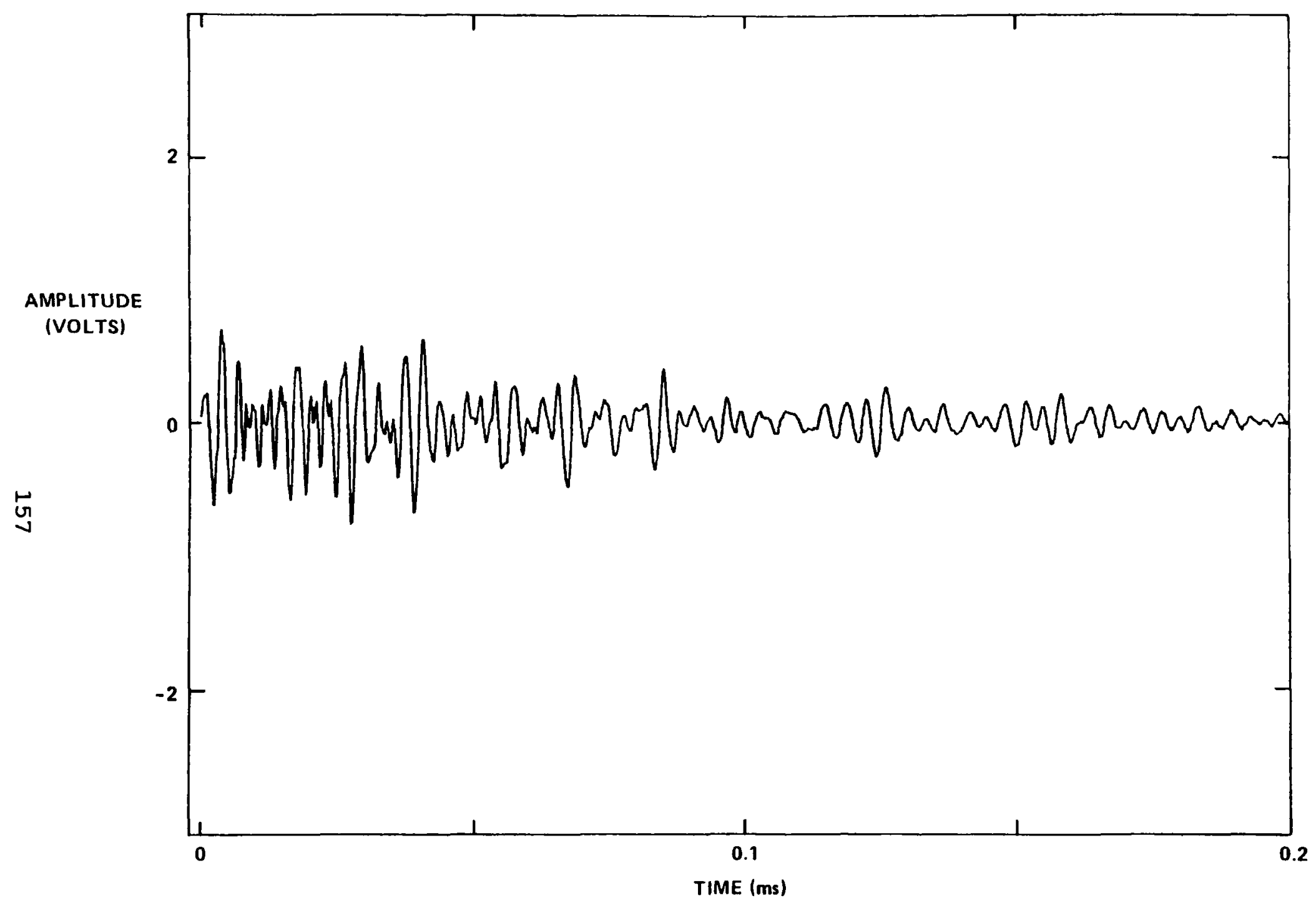

Figure 5.10(a): A typical acousto-ultrasonic waveform for a specimen with
void area 


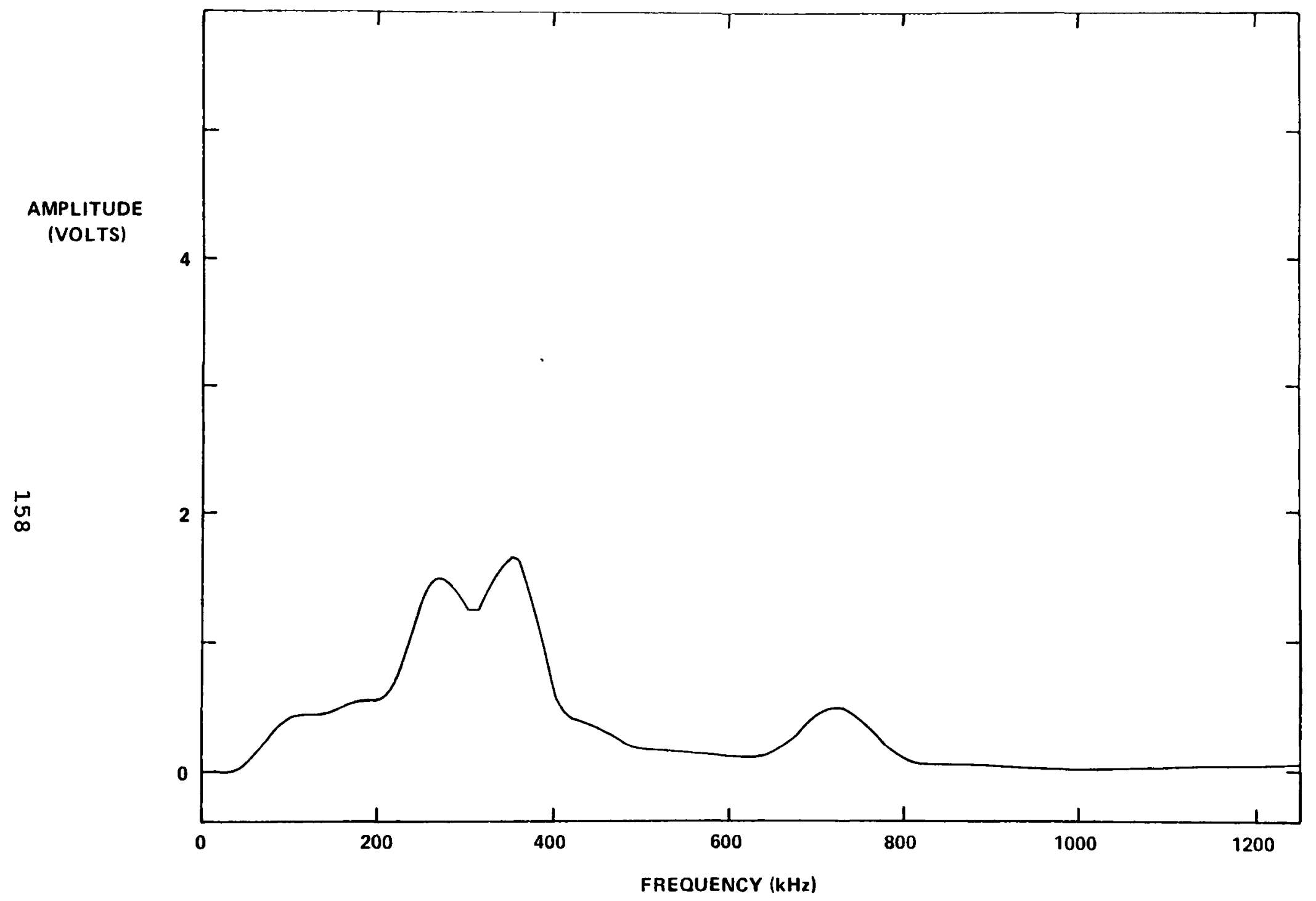

Figure 5.10(b): Frequency spectrum of the acousto-ultrasonic waveform shown in Figure 5.10(a) 


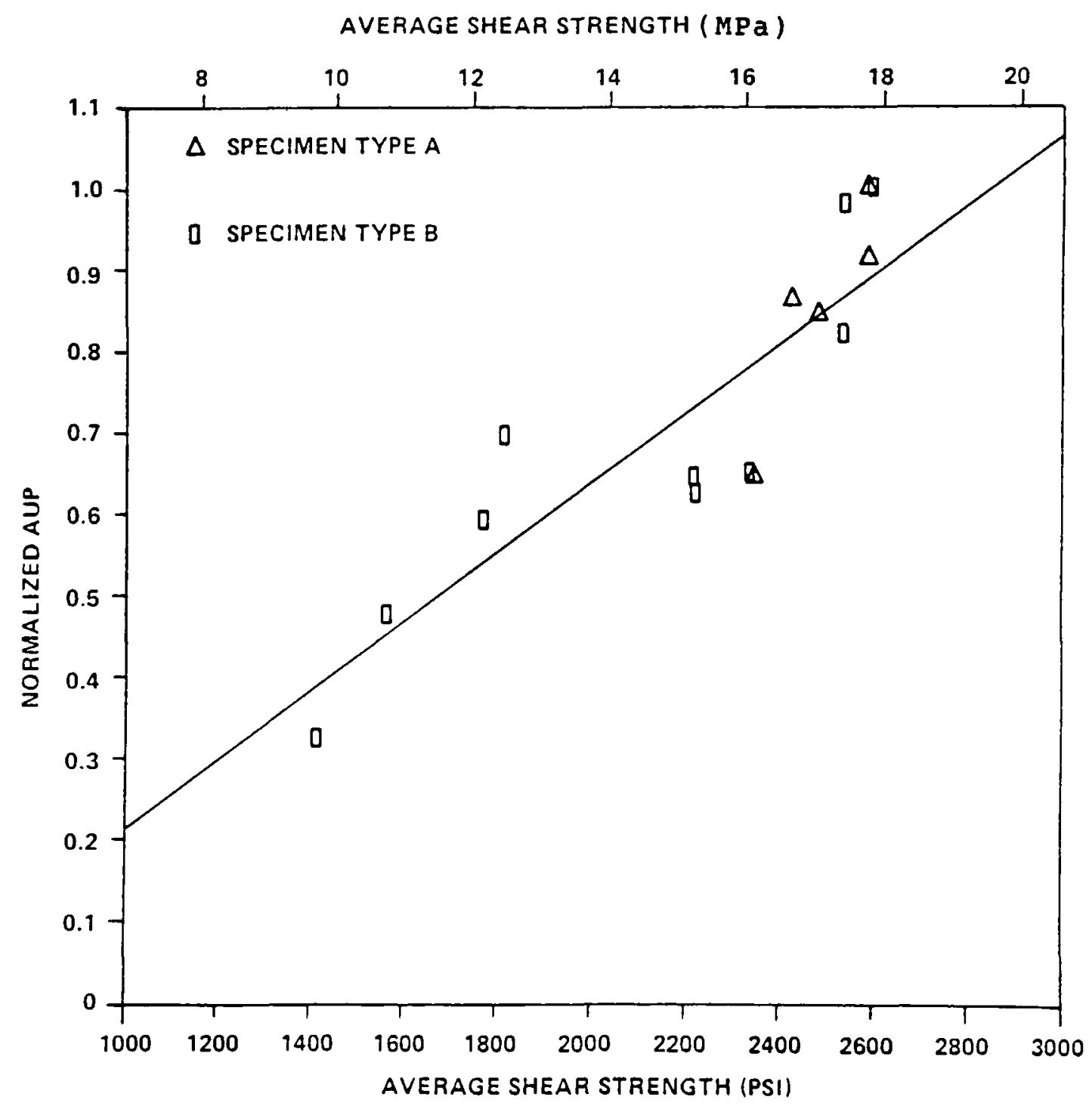

Figure 5.11: The normalized acousto-ultrasonic parameter (AUP) vs the average shear strength for specimens with voids 


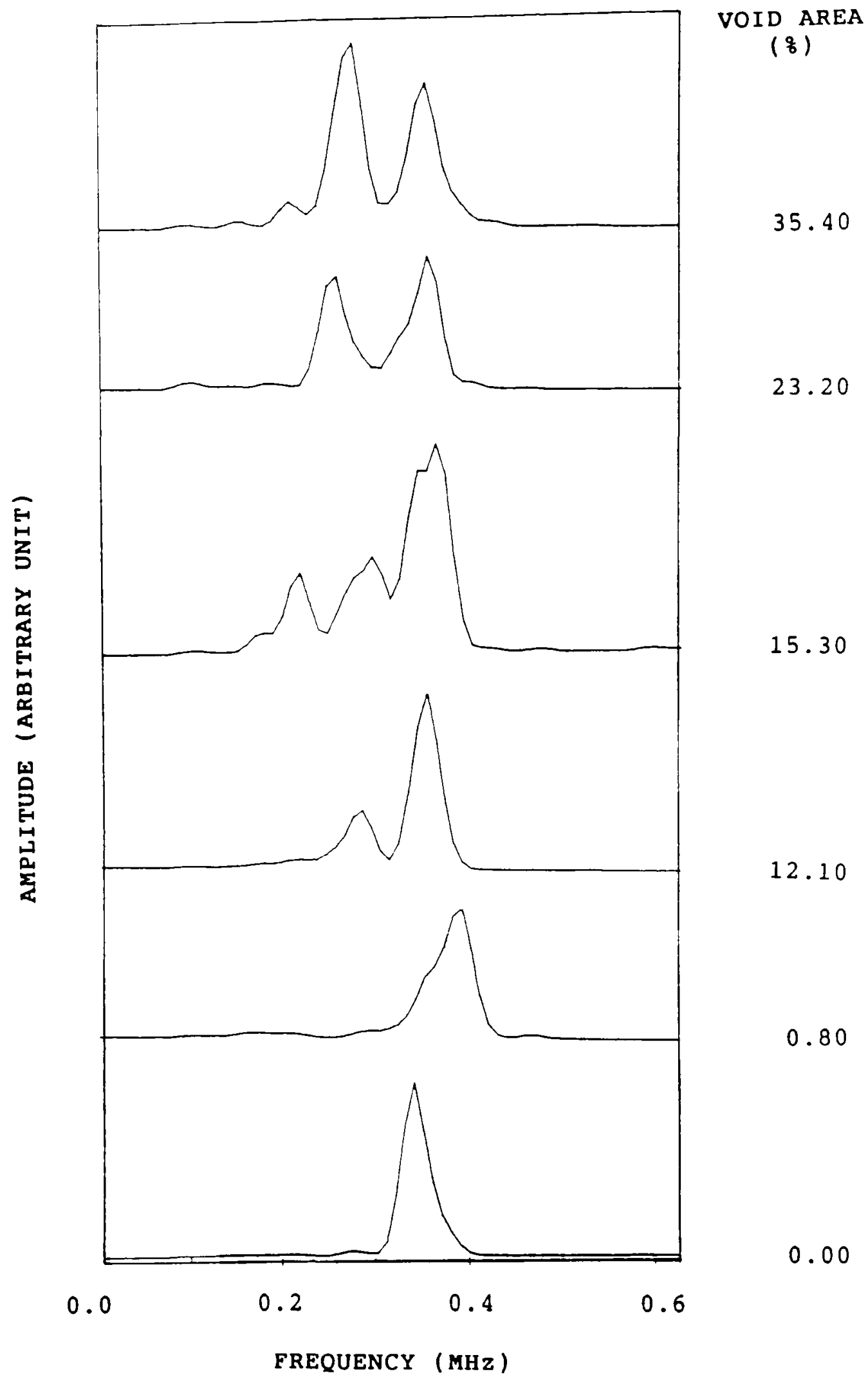

Figure 5.12 : The change in frequency spectra of the acousto-ultrasonic signals from bonded composite joint specimens with increasing void size 


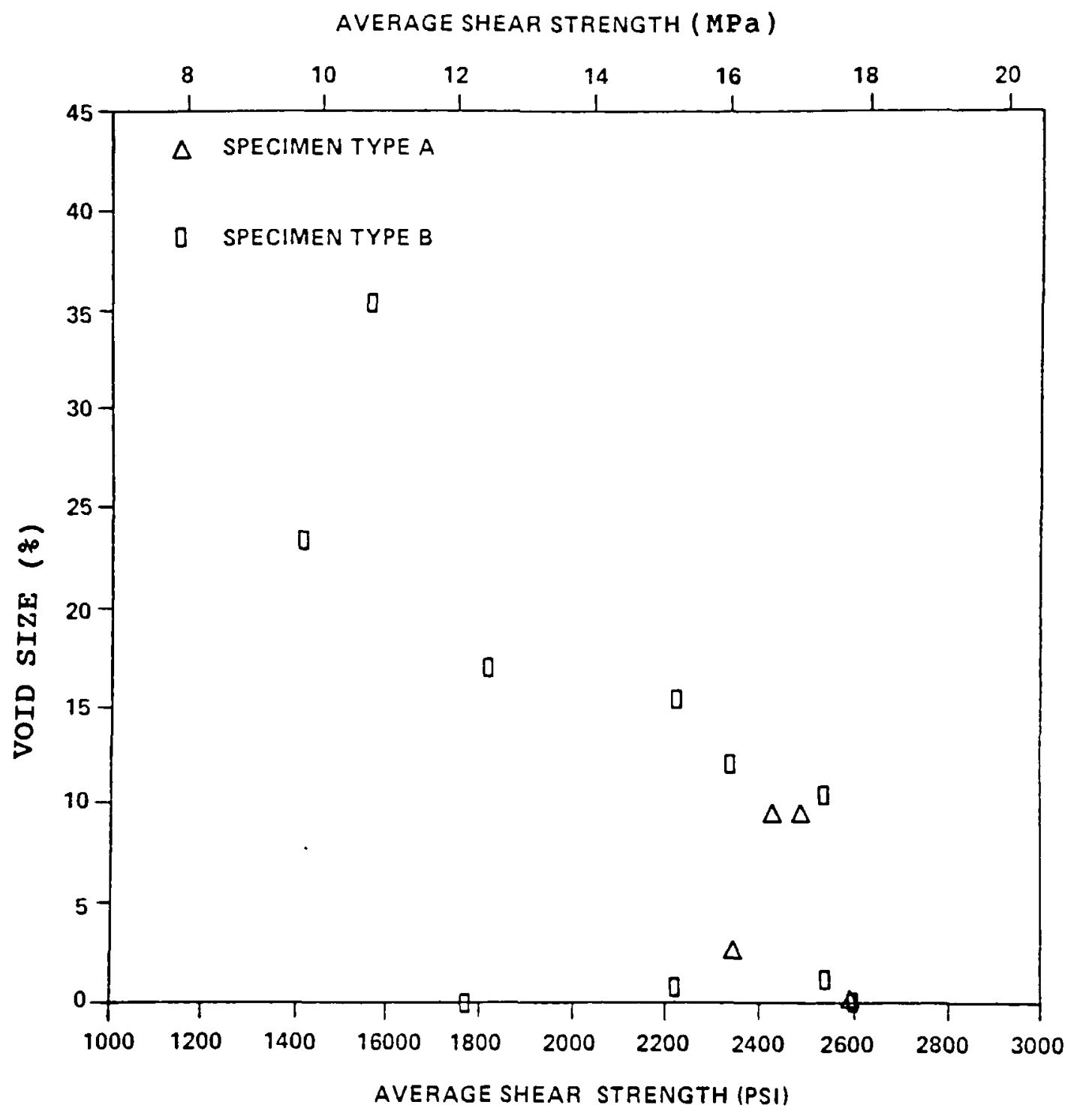

Figure 5.13: The average shear strength vs the void area for single lap composite joint specimens 


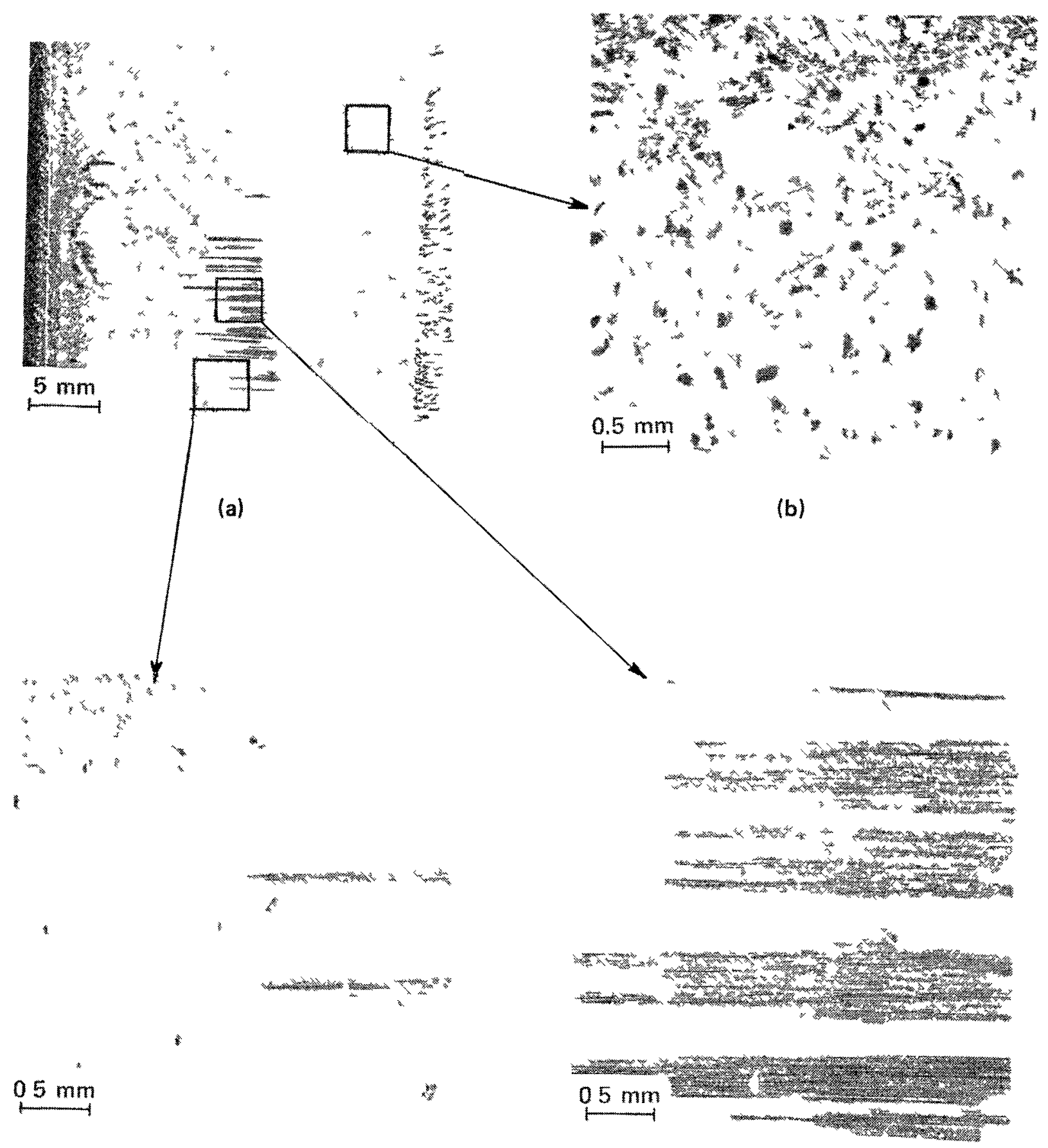

(c)

(d)

Figure 5.14: A typical fracture surface of a specimen without void area; (b) cohesive failure in the adhesive; (c) interfacial failure; (d) fibre pull-off 


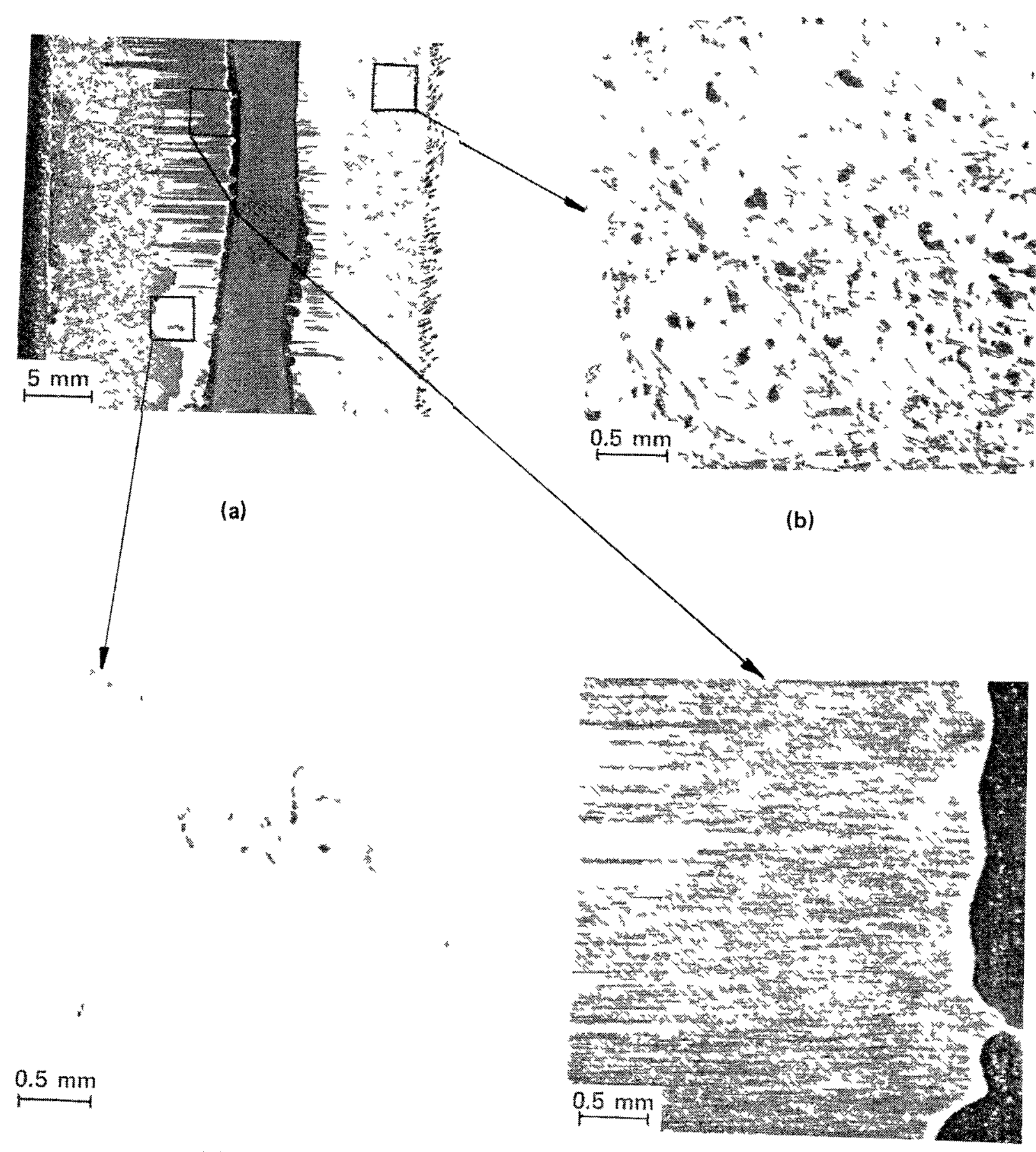

(c)

(d)

Figure 5.15: A typical fracture surface of a specimen with void area across the joint; (b) cohesive failure in the adhesive; (c) interfacial failure; (d) fibre pull- 


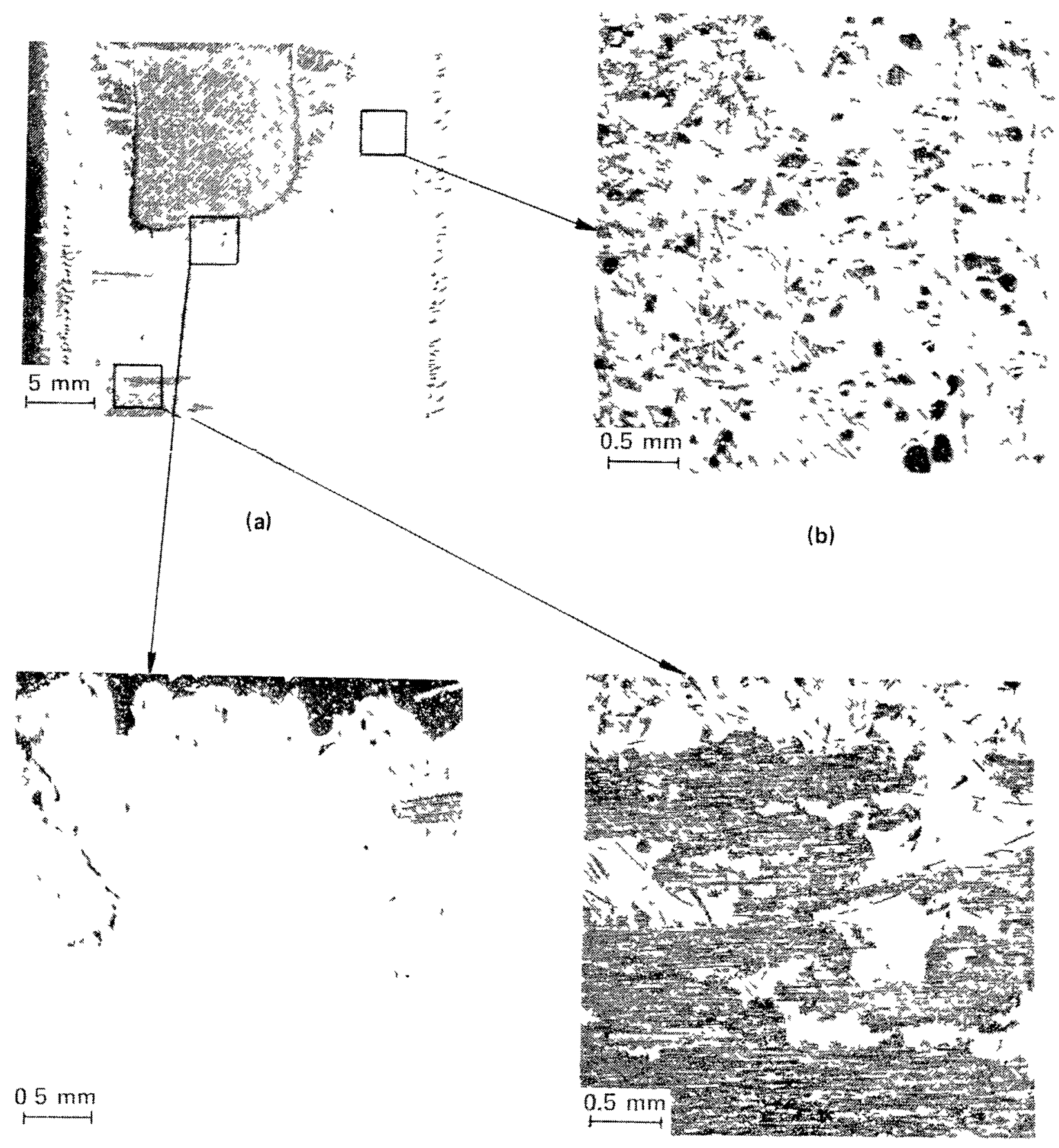

(c)

(d)

Figure 5.16: A typical fracture surface of a specimen with void area at the edge of the joint; (b) cohesive failure in the adhesive; (c) interfacial failure; (d) fibre pull-off 


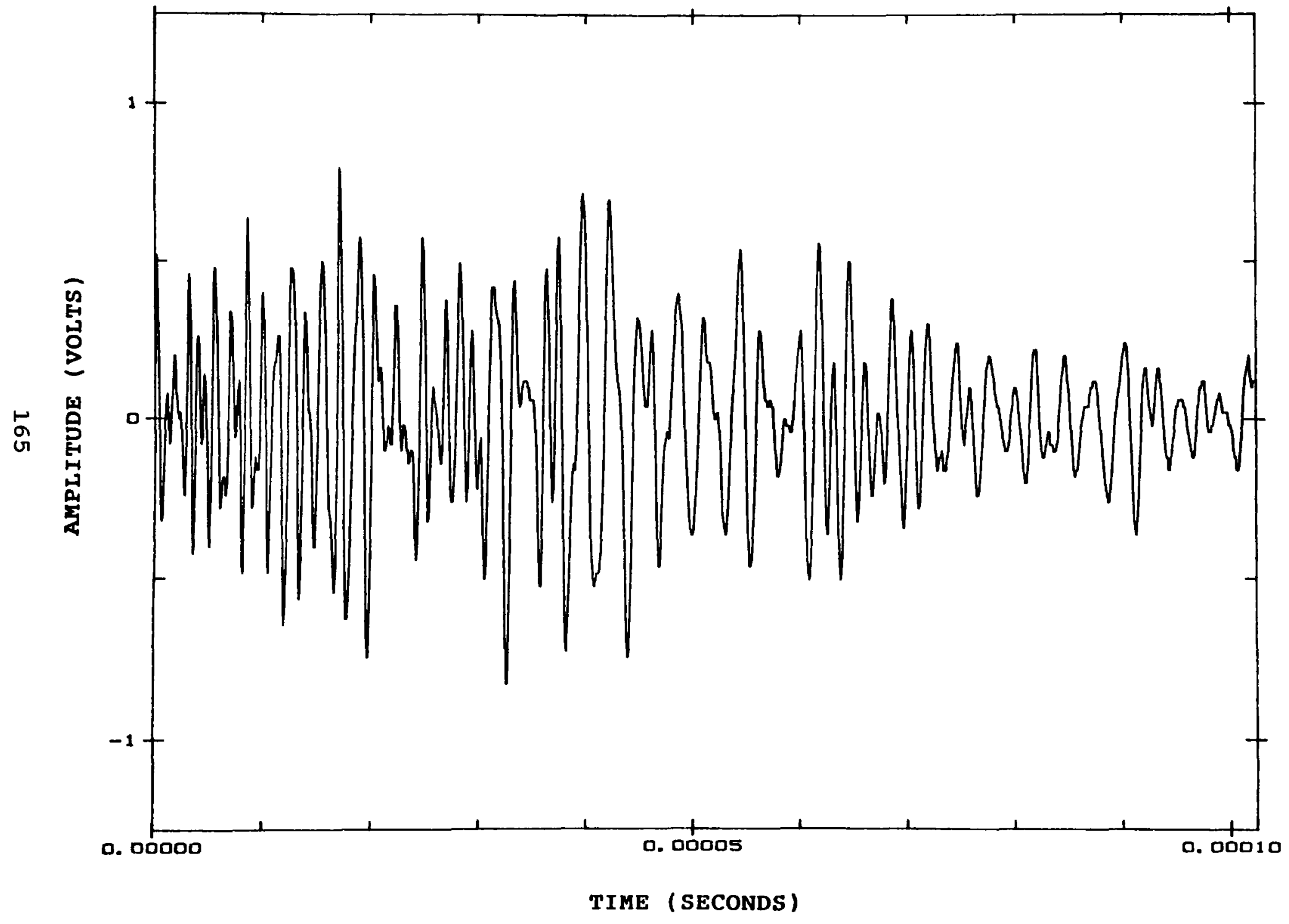

Figure 5.17 : An acousto-ultrasonic waveform for a typical specimen without unbonded area 


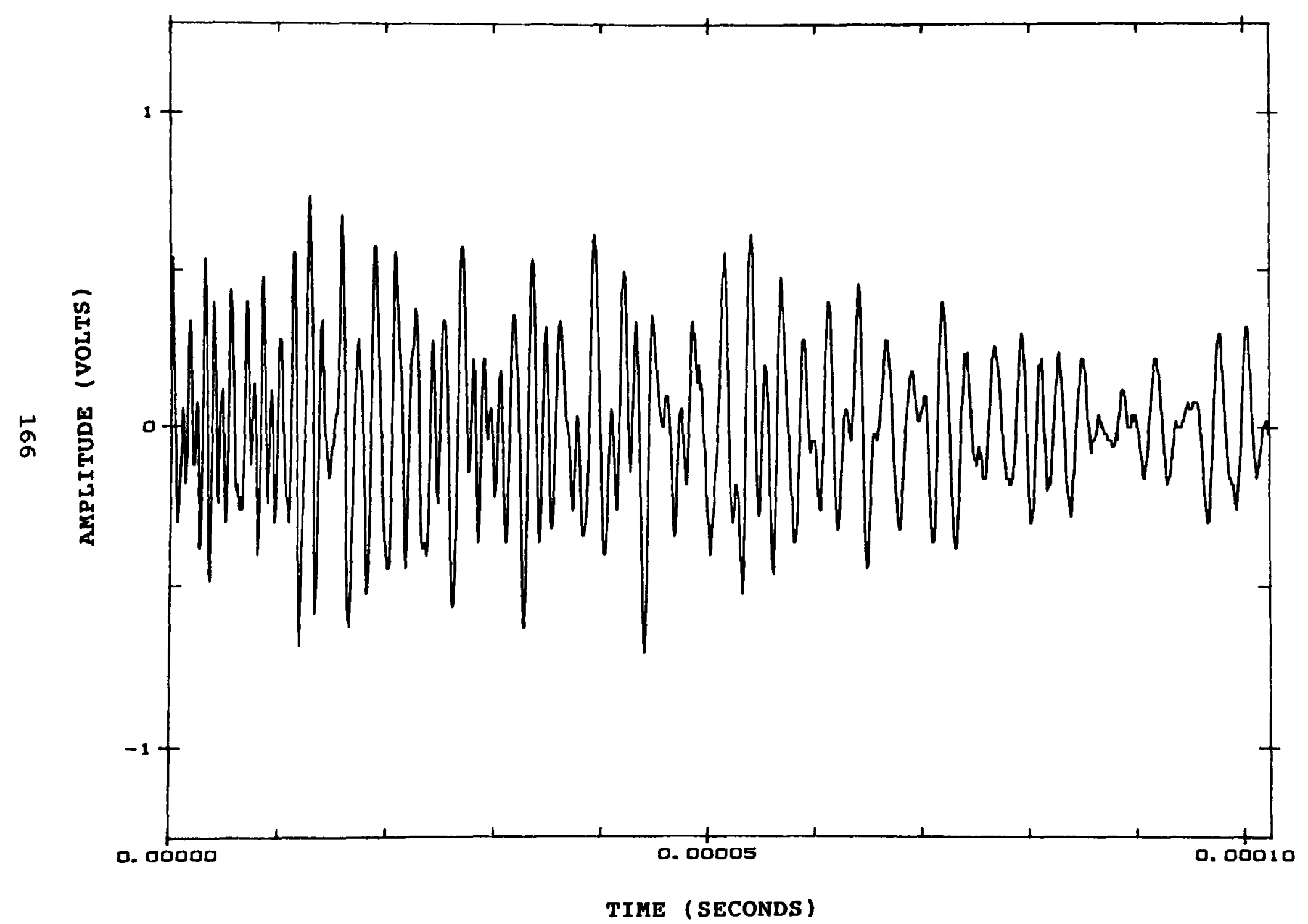

Figure 5.18: An acousto-ultrasonic waveform for a typical specimen with unbonded area 


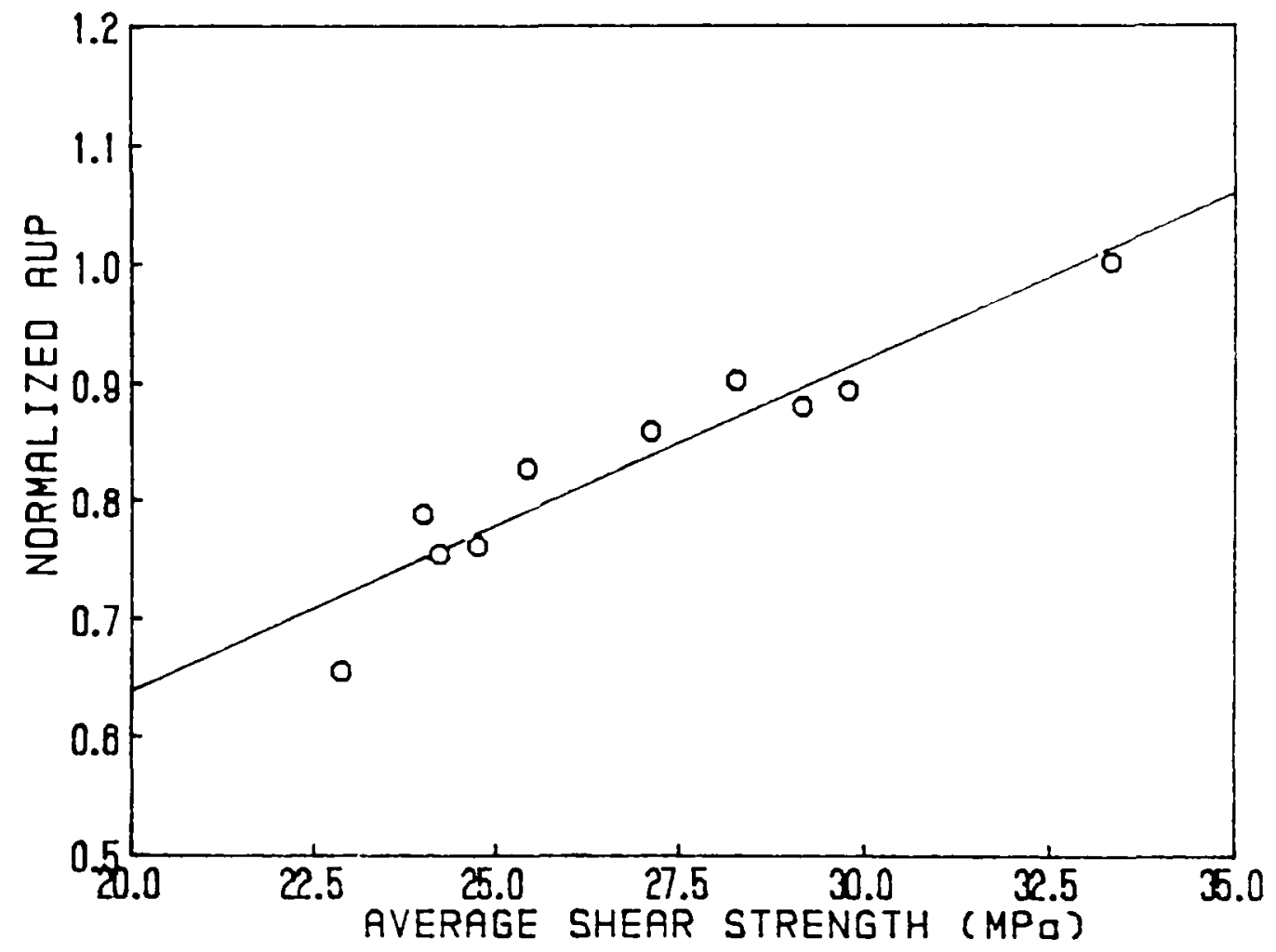

Figure 5.19 : The correlation between the normalized acousto-ultrasonic parameter (AUP) and the average shear strength of the specimens with unbonds 


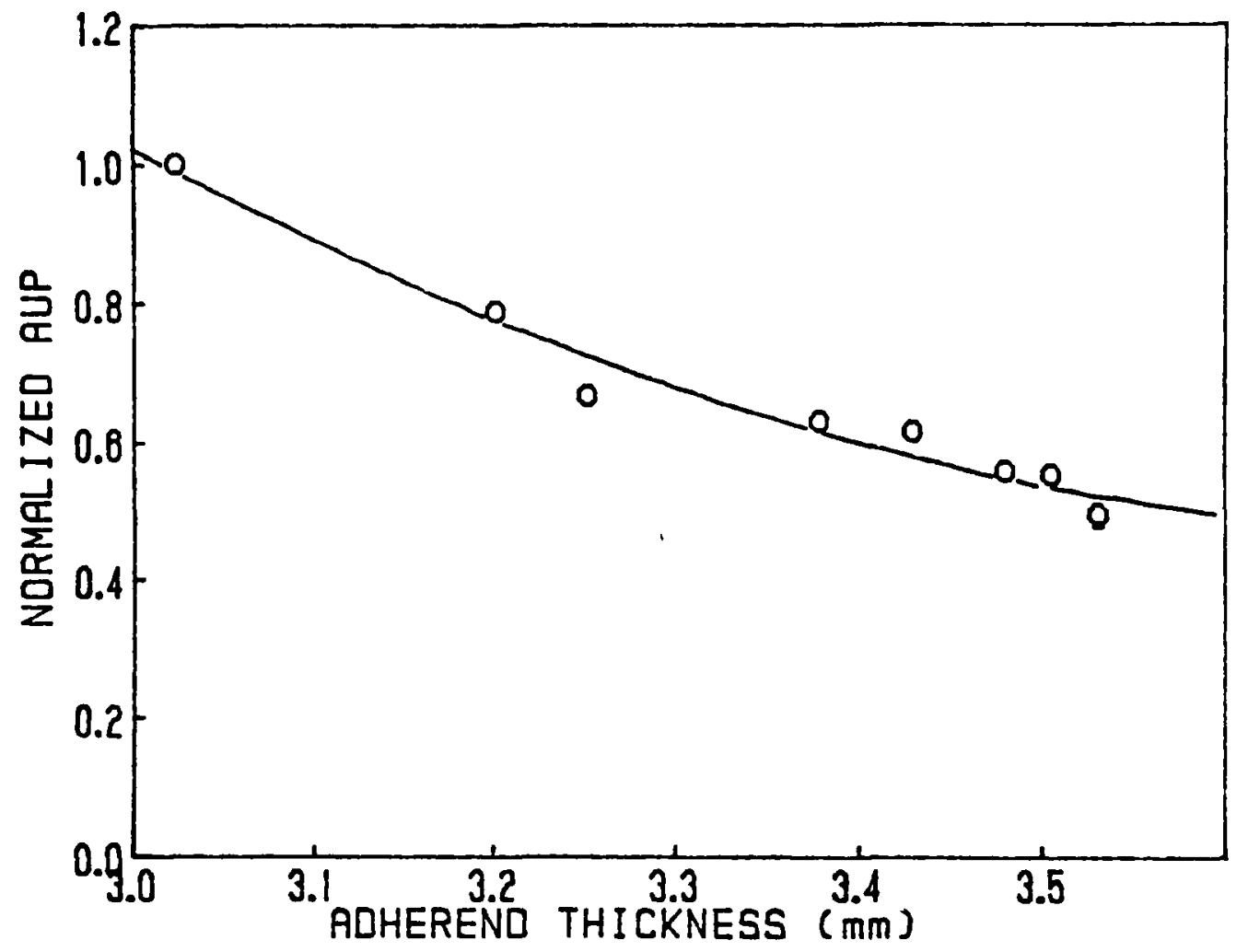

Figure 5.20: The change in normalized AUP with respect to the variation in the adherend thickness 


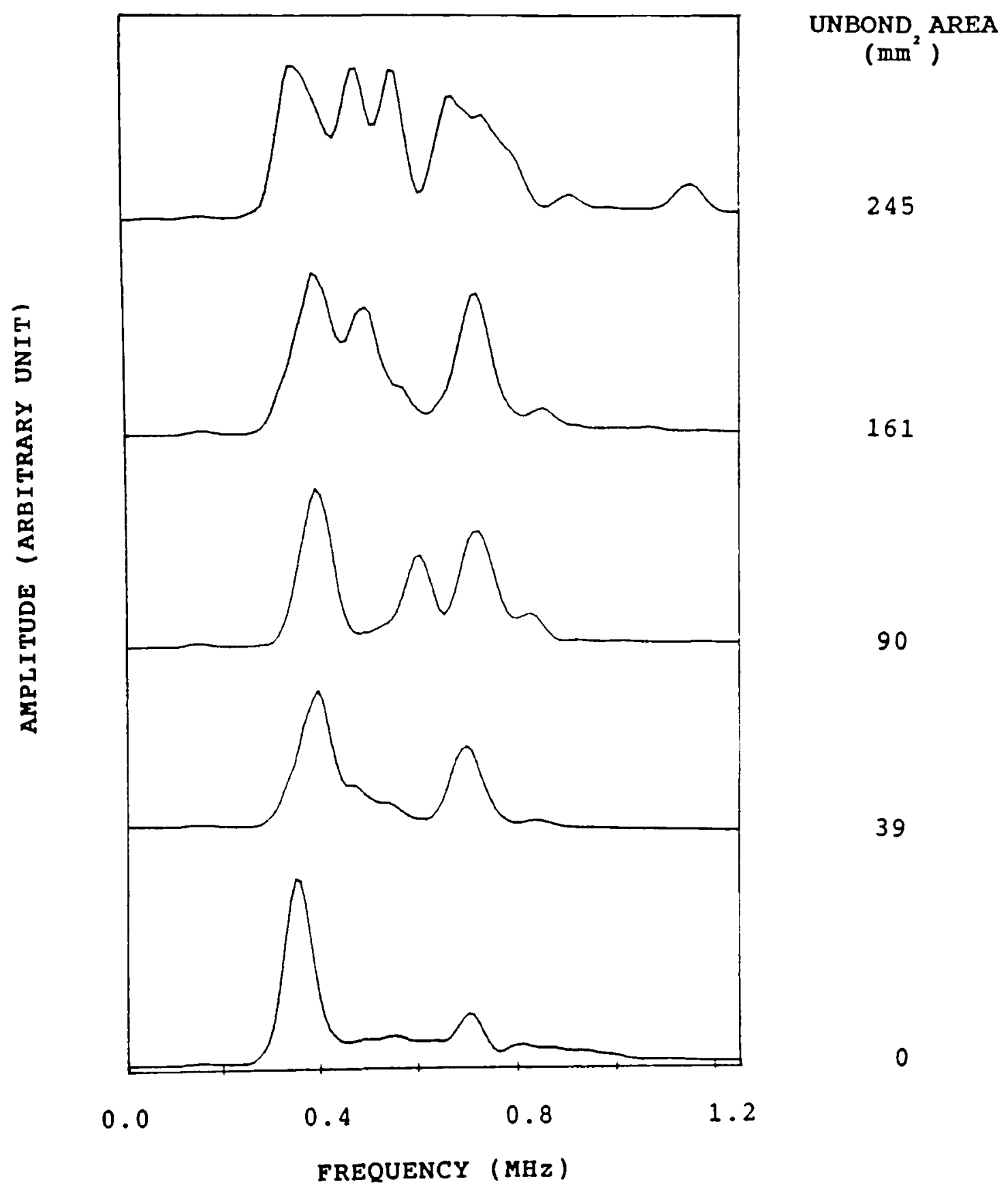

Figure 5.21: The change in the frequency spectra of the acousto-ultrasonic signals from bonded joint specimens with increasing unbonded area 


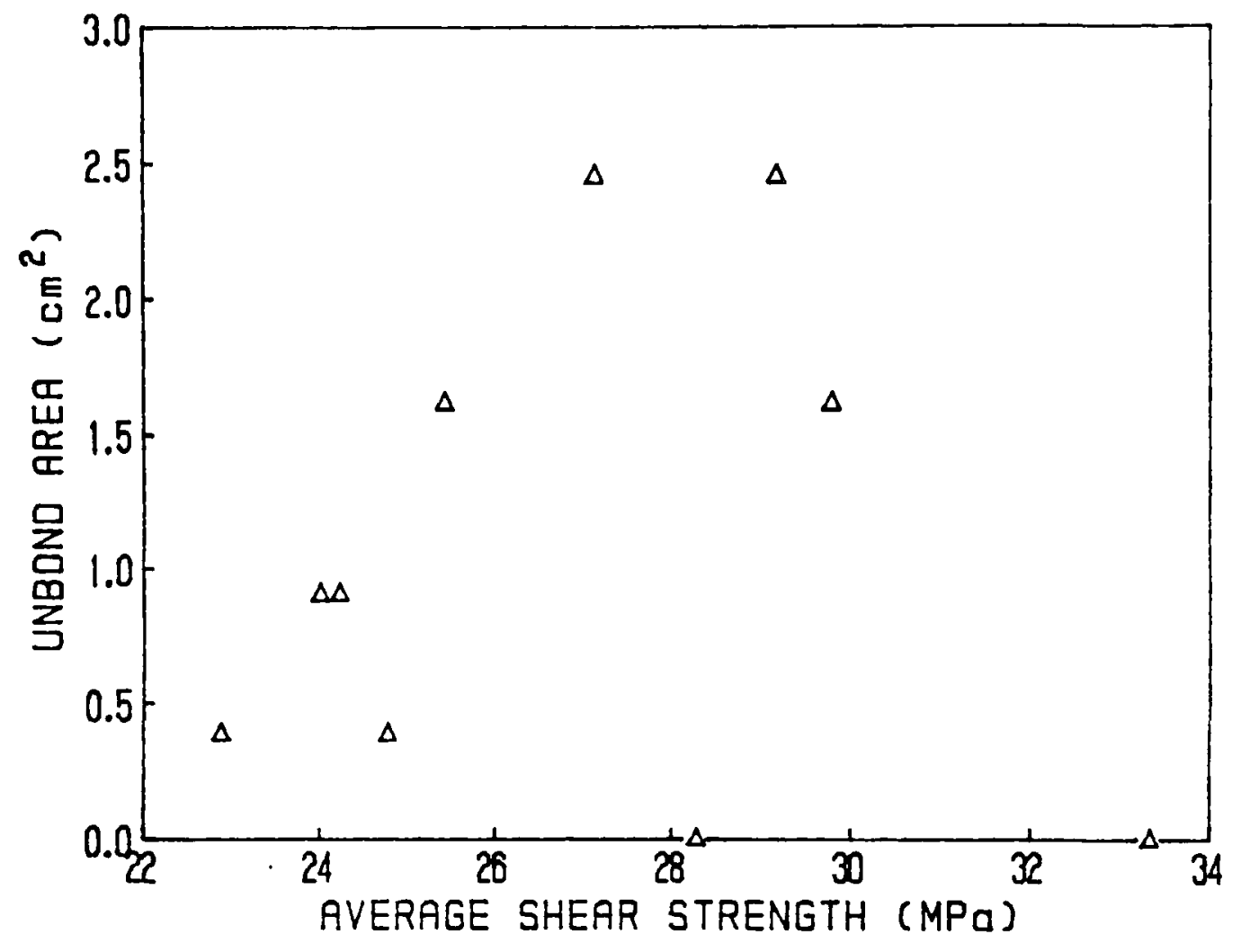

Figure 5.22 : The average shear strength vs the unbonded area for single lap composite joint specimens 

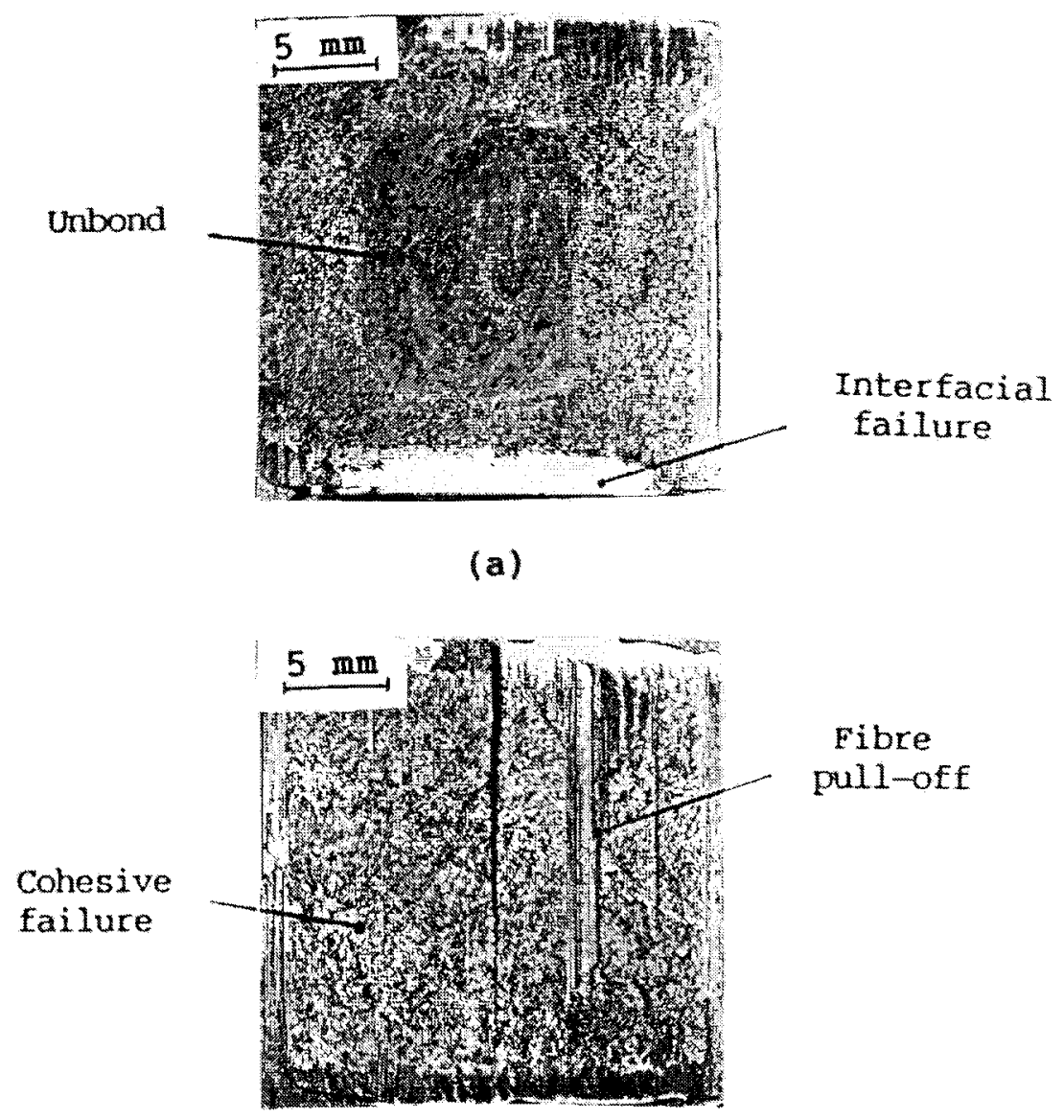

(b)

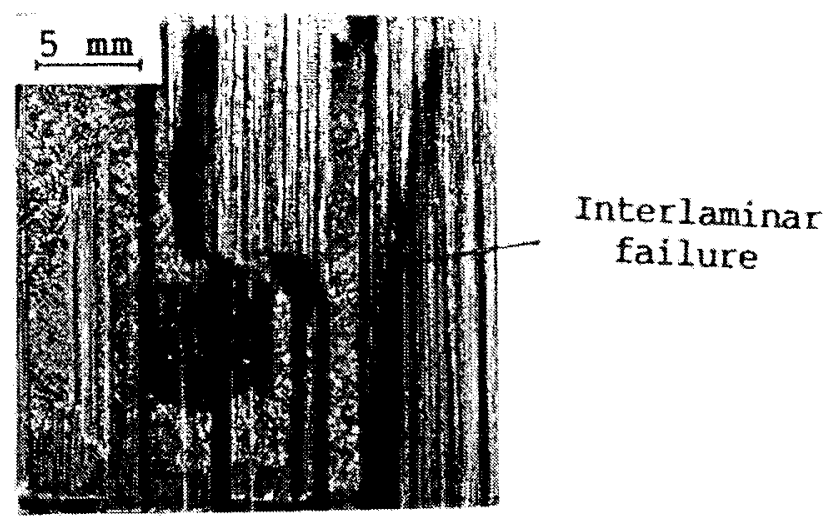

(c)

Figure 5.23 : Photographs of typical fracture surfaces for specimens with unbonds showing the different failure modes 


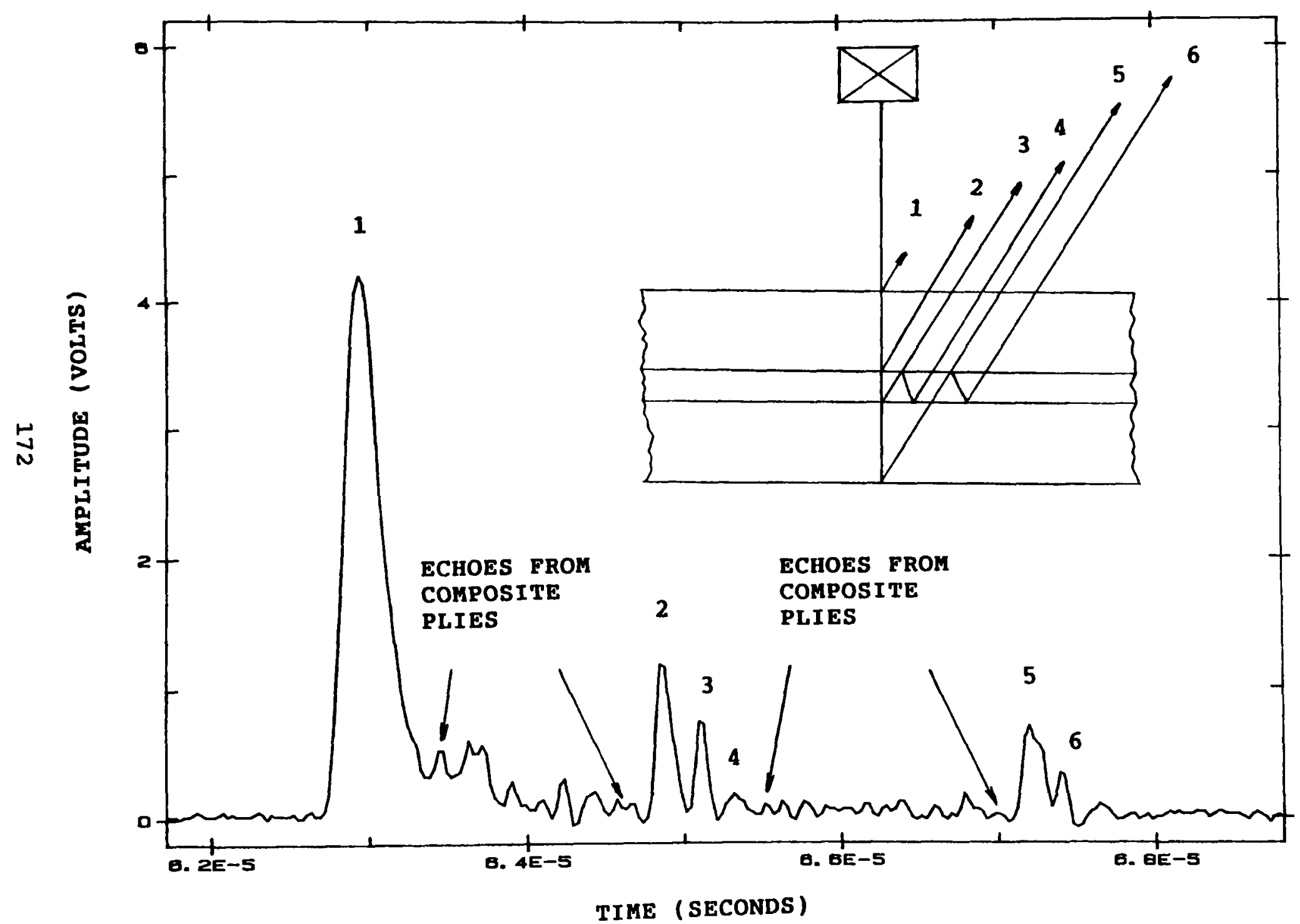

Figure 5.24: A typical ultrasonic pulse-echo signal for an adhesively bonded joint in composites 


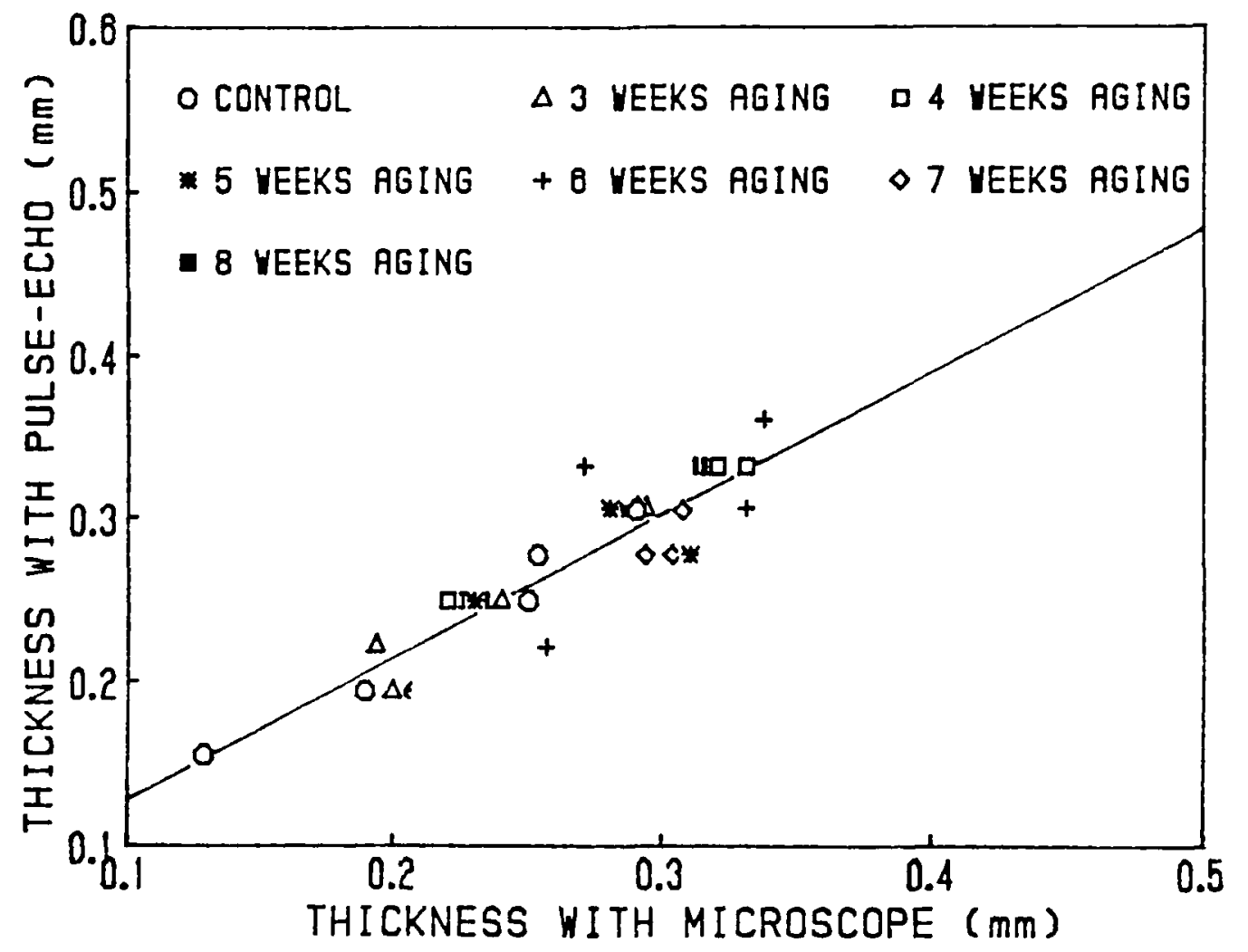

Figure 5.25: Adhesive thicknesses of specimens measured with ultrasonic pulse-echo method vs those measured with an optical microscope 

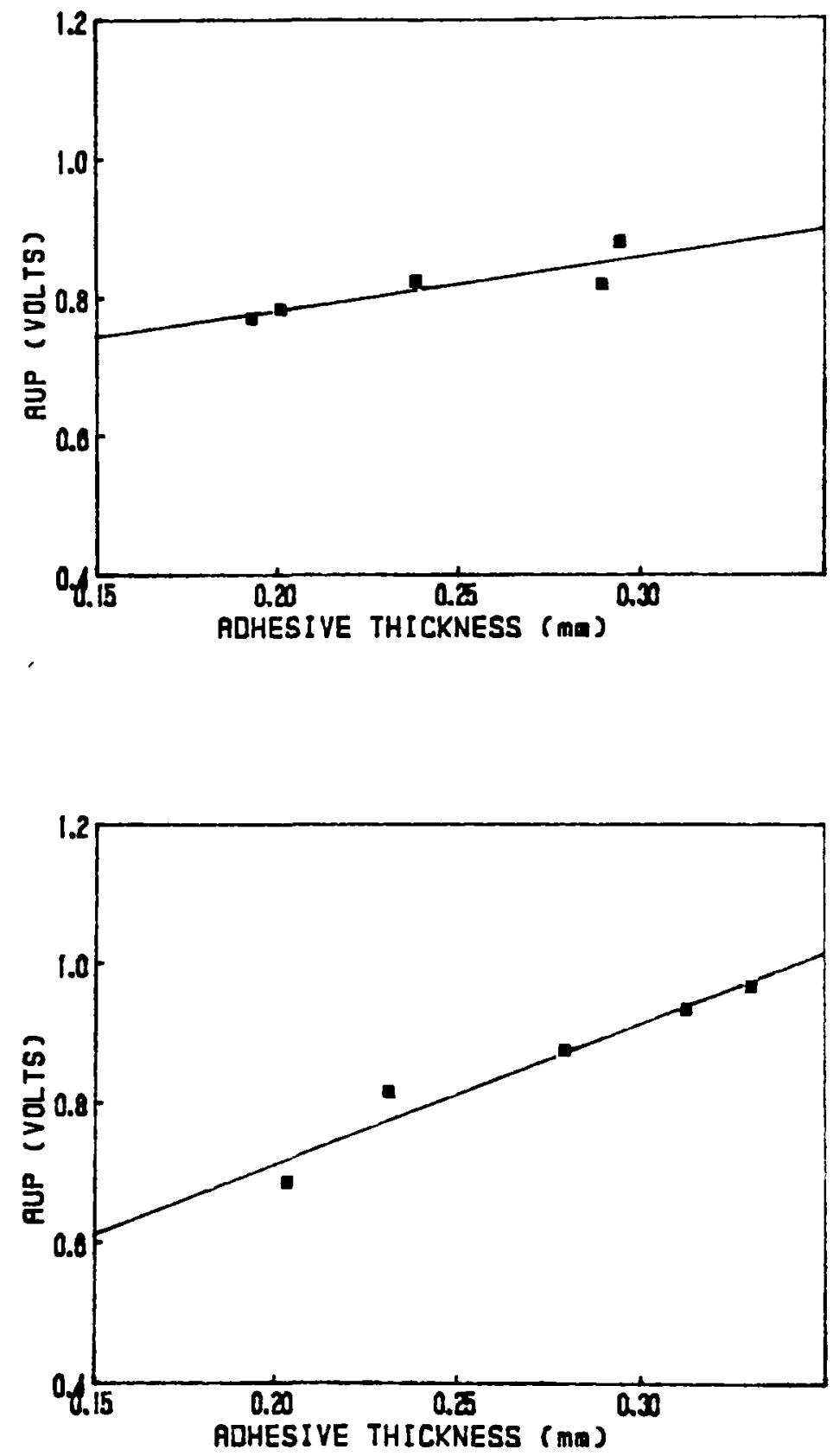

Figure 5.26: The effect of adhesive thickness on the AUP for specimens with (a) 3 weeks of aged adhesive; (b) 5 weeks of aged adhesive 


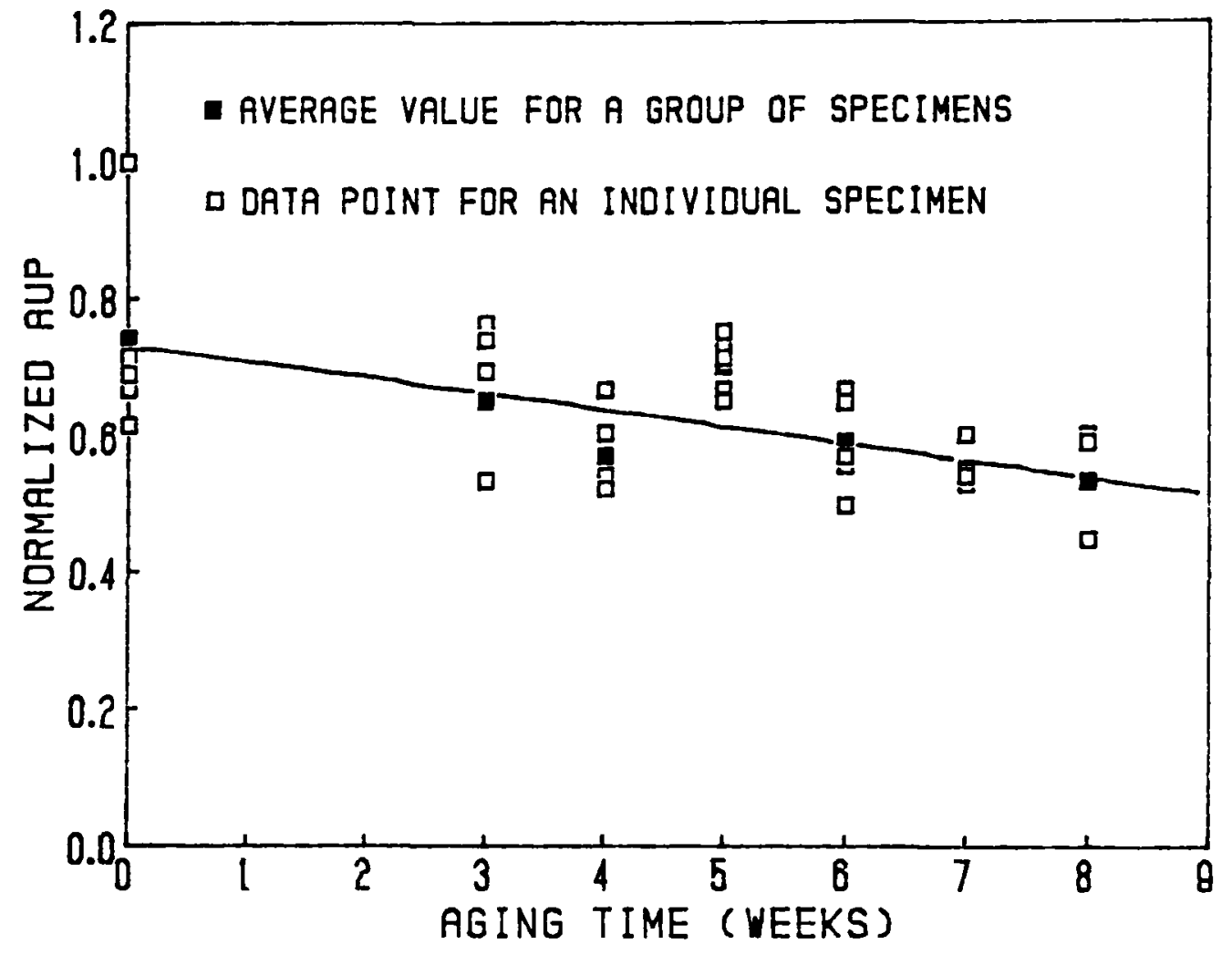

Figure 5.27 : The change in normalized AUP (Equation 5.1) as a function of aging time 


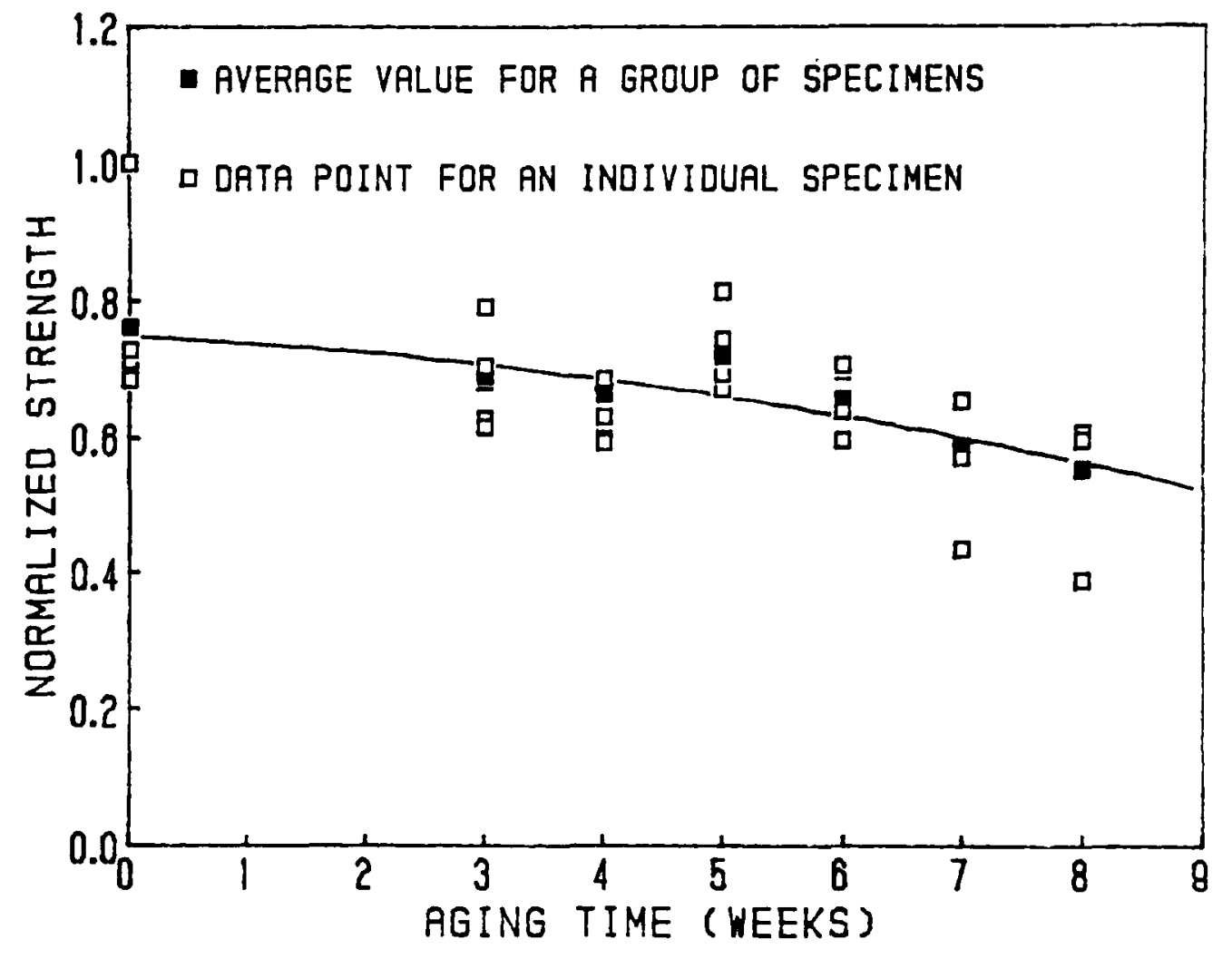

Figure 5.28: The change in normalized joint strength (Equation 5.2) as a function of aging time 


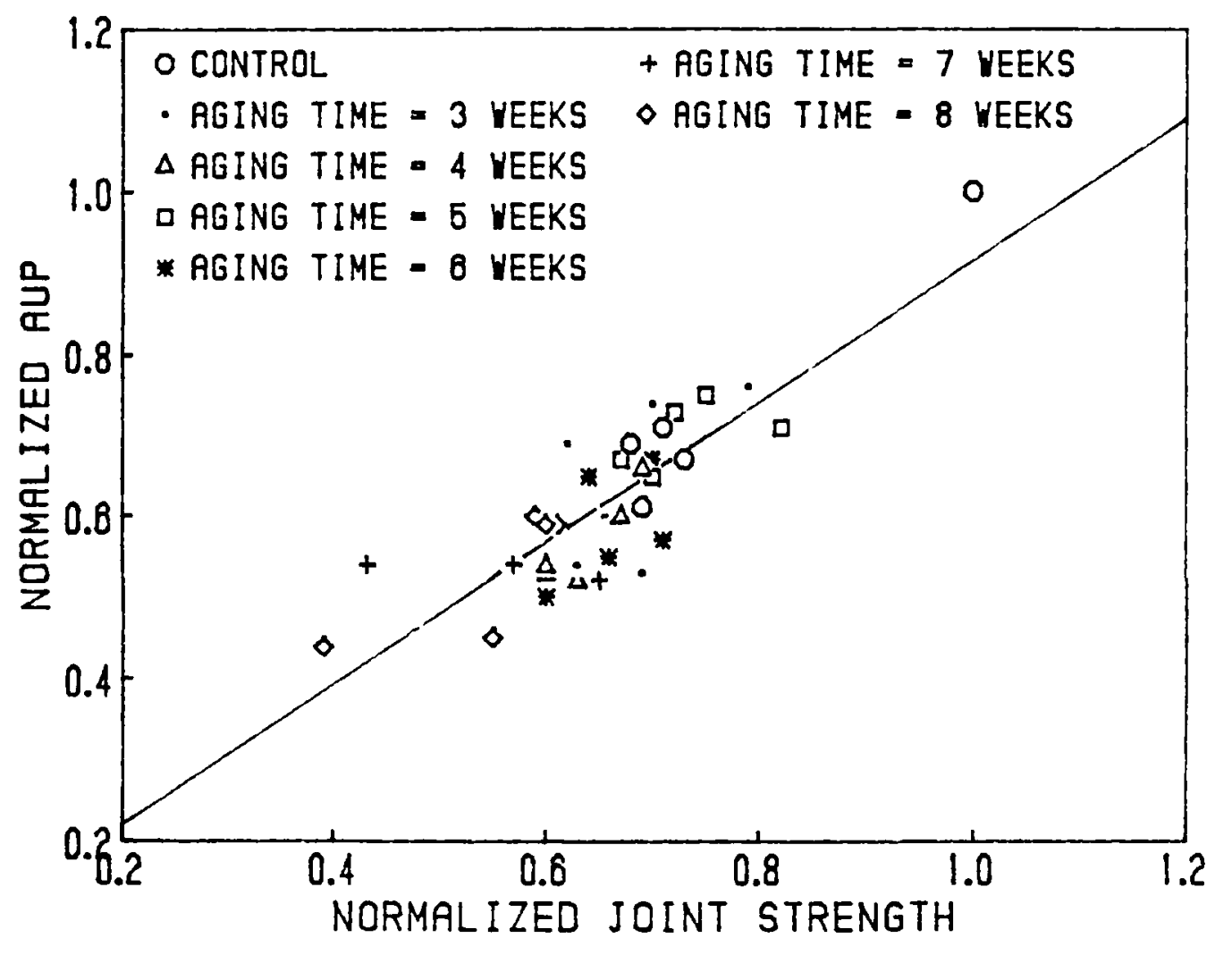

Figure 5.29: Plot of the normalized AUP vs the normalized joint strength 


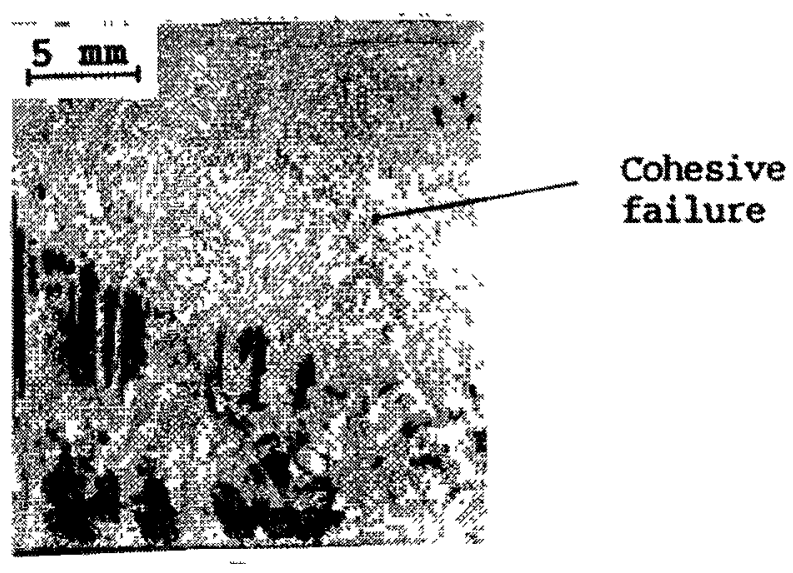

(a)

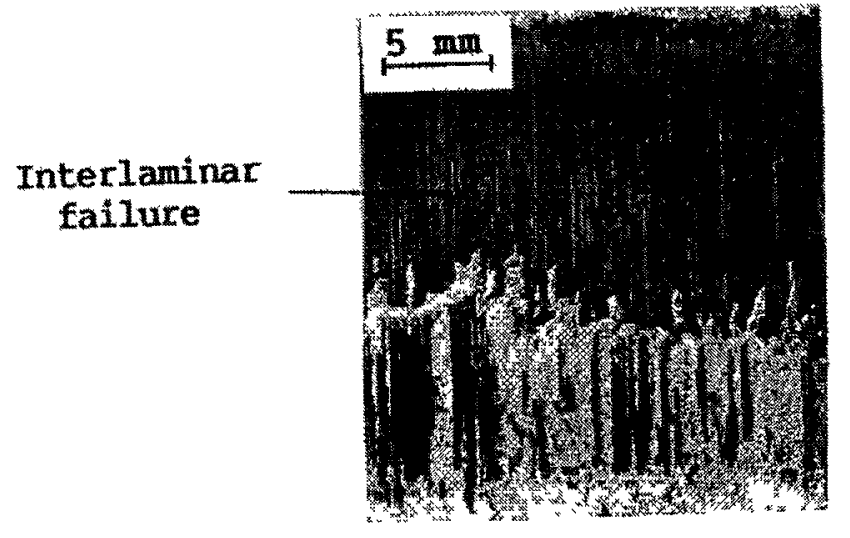

(b)

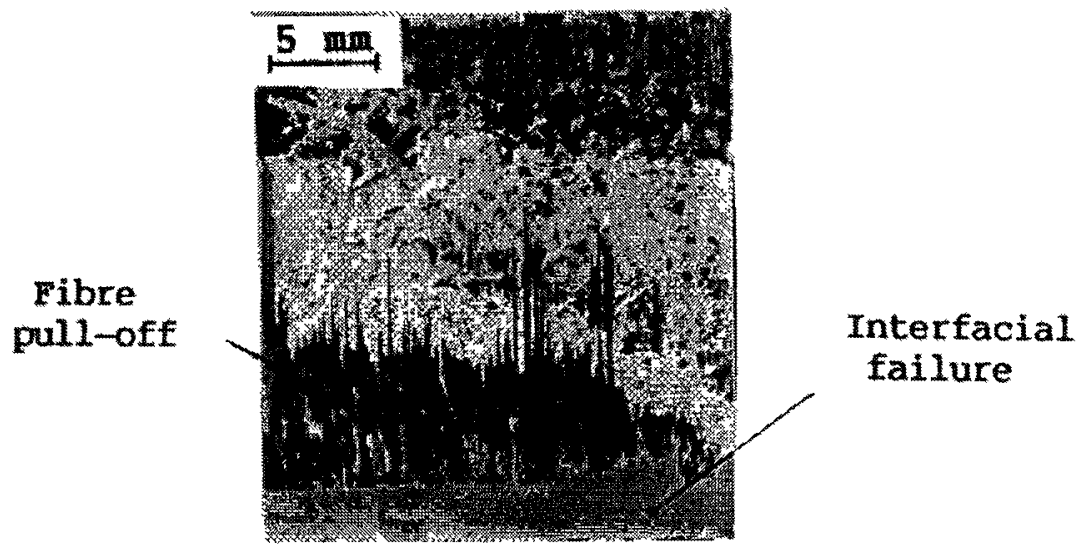

(c)

Figure 5.30: Photographs of fracture surfaces for specimens with aged adhesive showing different failure modes 


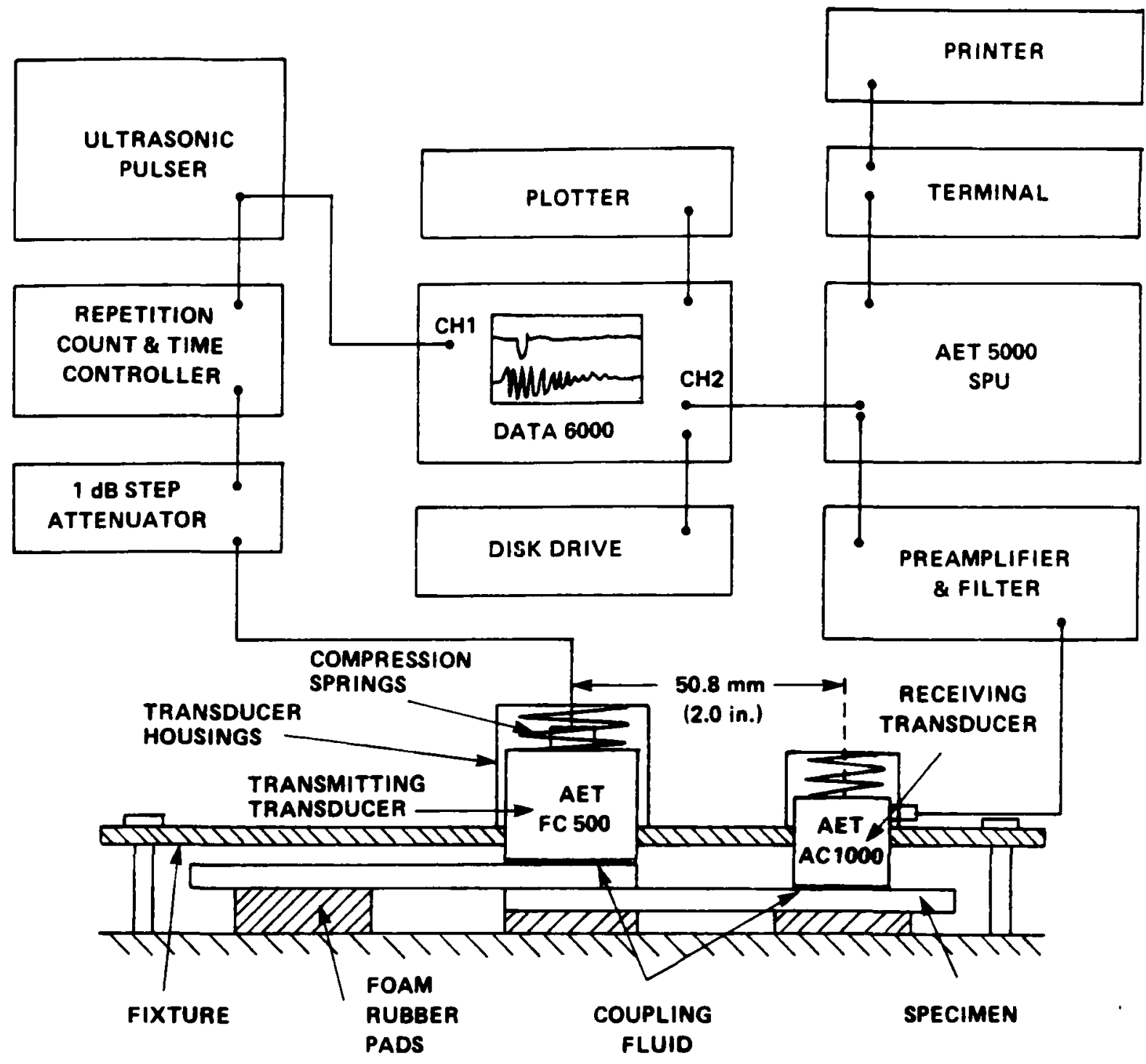

(a)

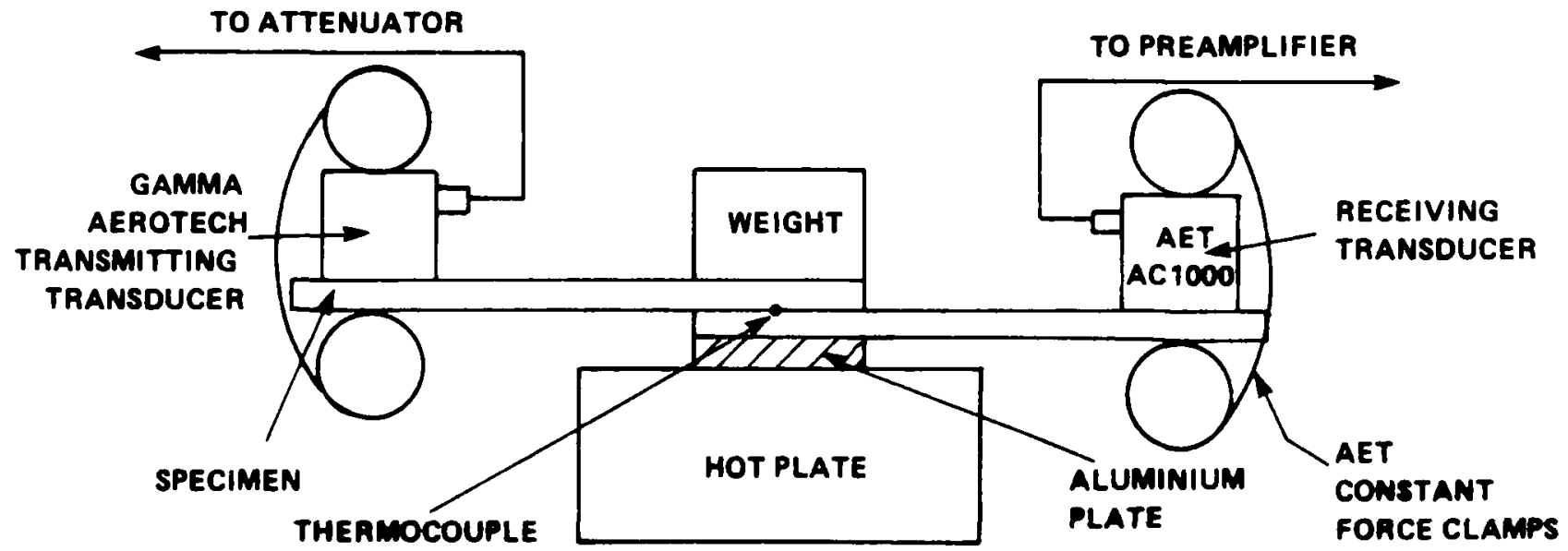

(b) Figure 6.1: Acousto-ultrasonic system (a) for room temperature $A U$ measurements and (b) for elevated temperature AU measurements 


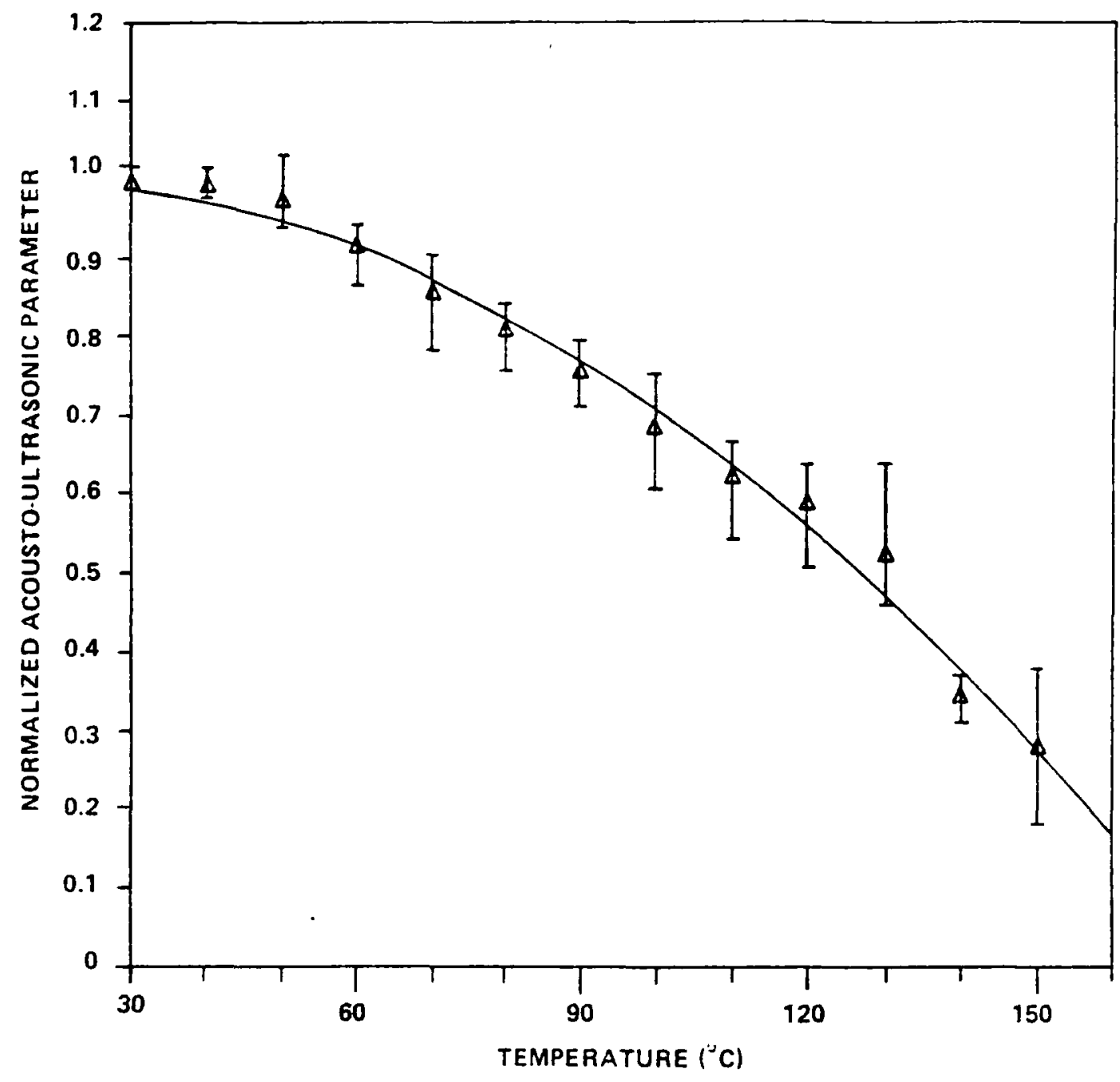

Figure 6.2 : The change in normalized acousto-ultrasonic parameter (AUP) with increasing temperature. The normalized AUP is the ratio of $A U P$ at a given temperature to the value of AUP at $30^{\circ} \mathrm{C}$. The solid line represents the best fit to the data points using a regression polynomial of order 2 


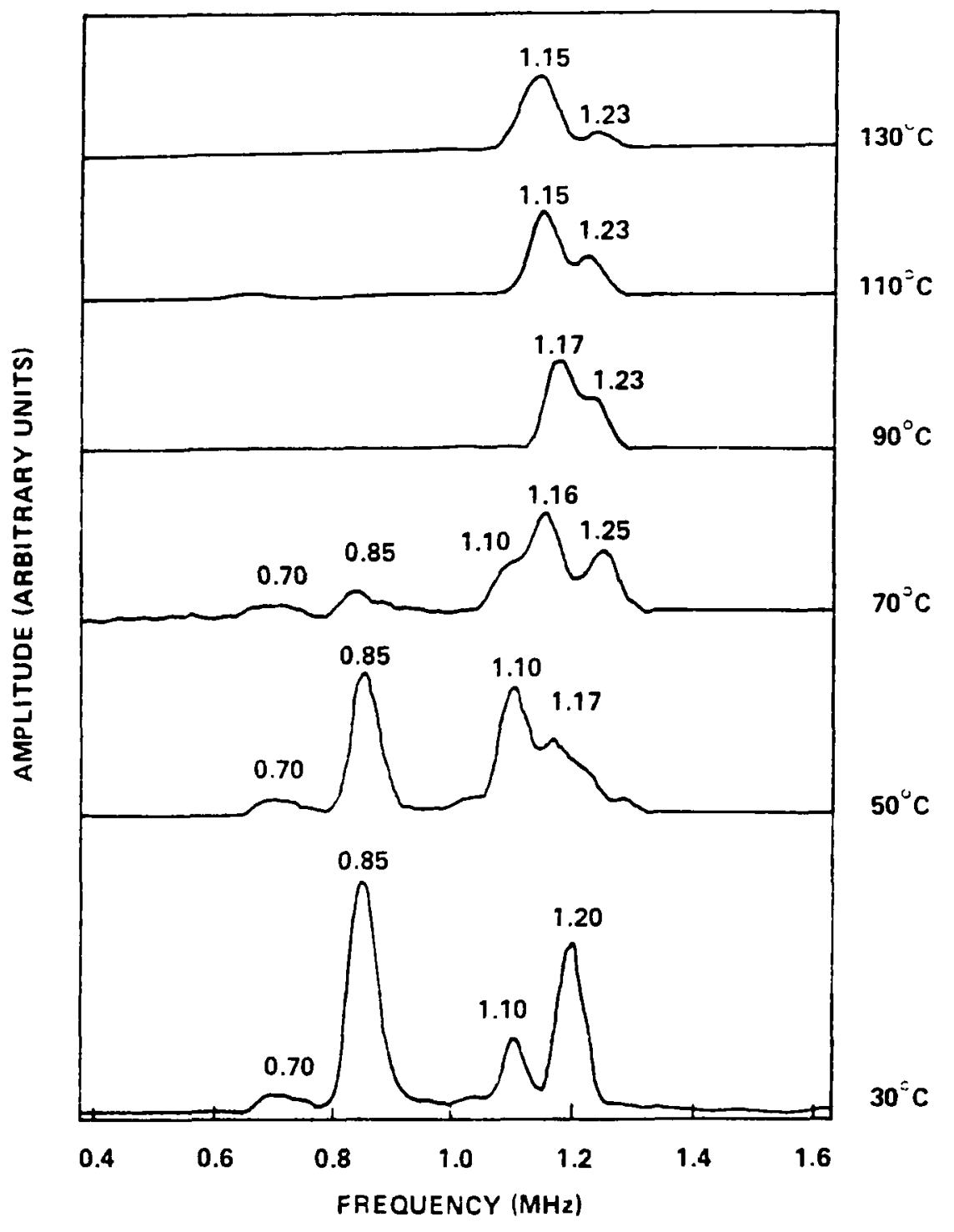

Figure 6.3: The change in frequency spectra of the acousto-ultrasonic signal from an adhesively bonded steel specimen with increasing temperature 


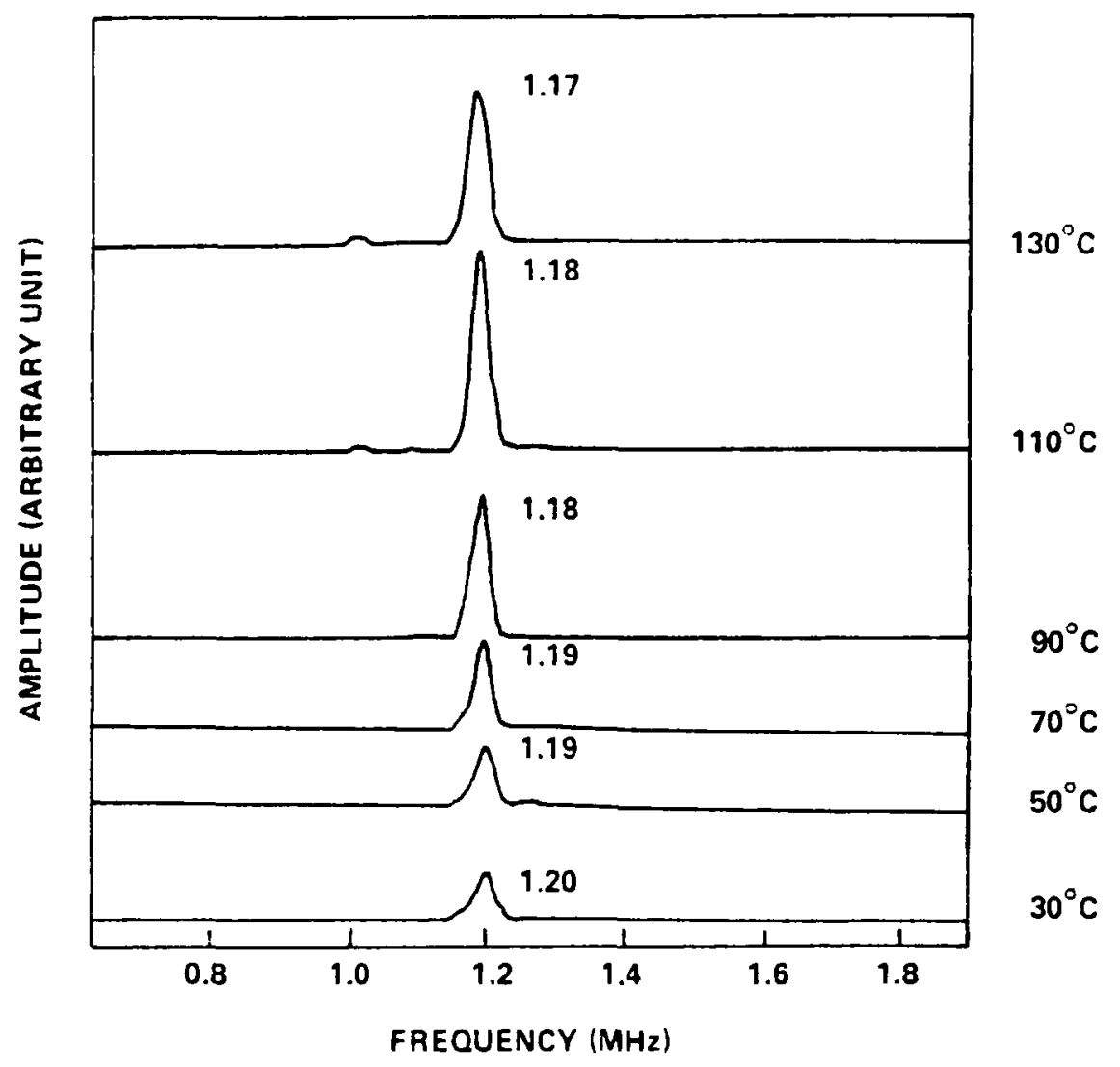

Figure 6.4: The frequency spectra of the acoustoultrasonic signal from a steel specimen without adhesive bond at different temperatures 


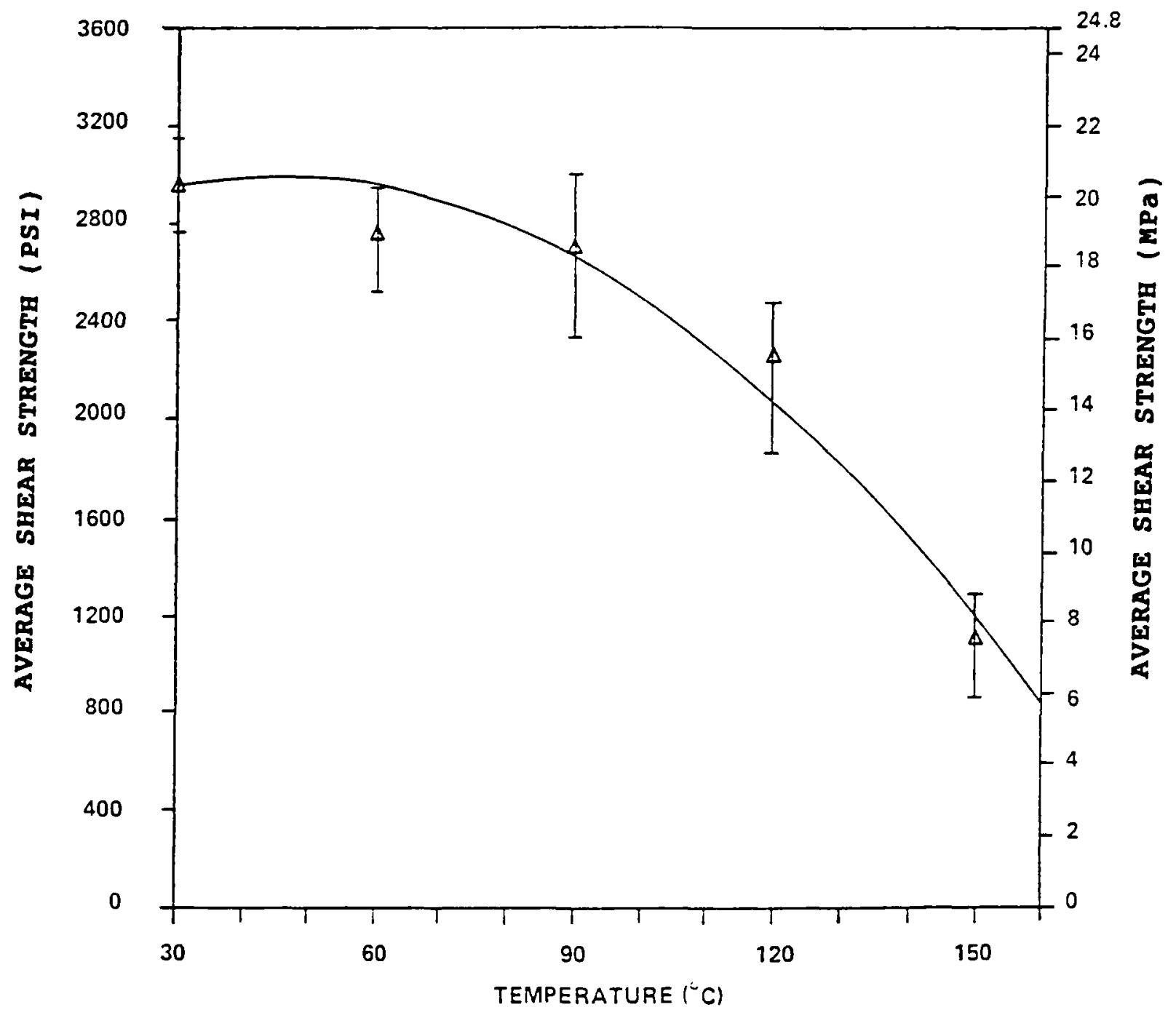

Figure 6.5: The change in average shear strength of the adhesively bonded steel specimens with increasing temperature. The solid line represents the best fit to the data points using a regression polynomial of order 2 


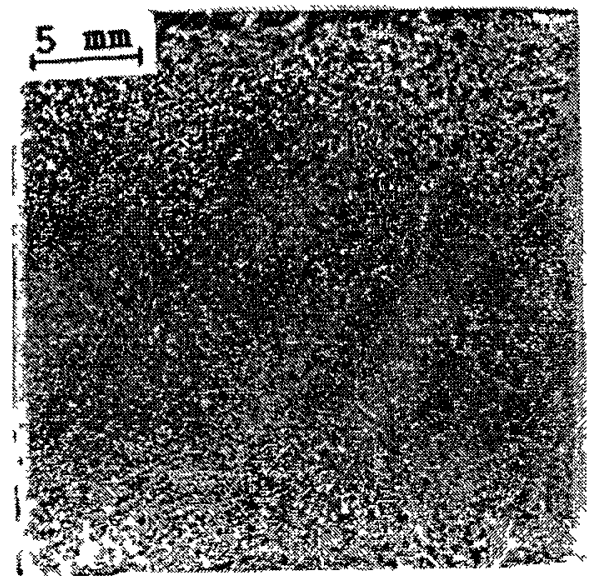

(a)

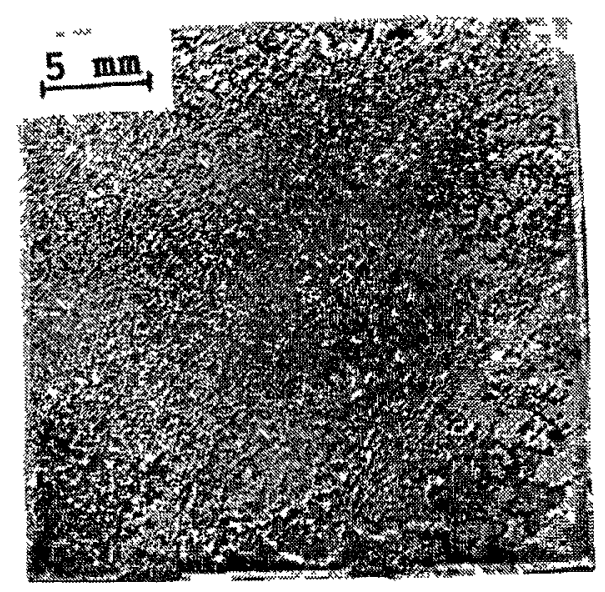

(c)

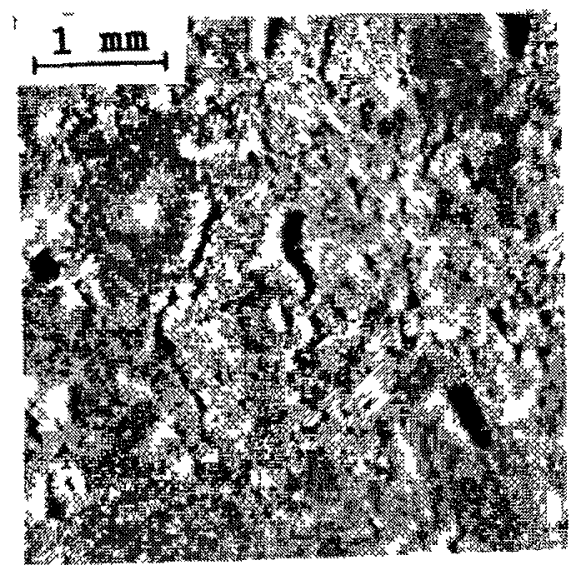

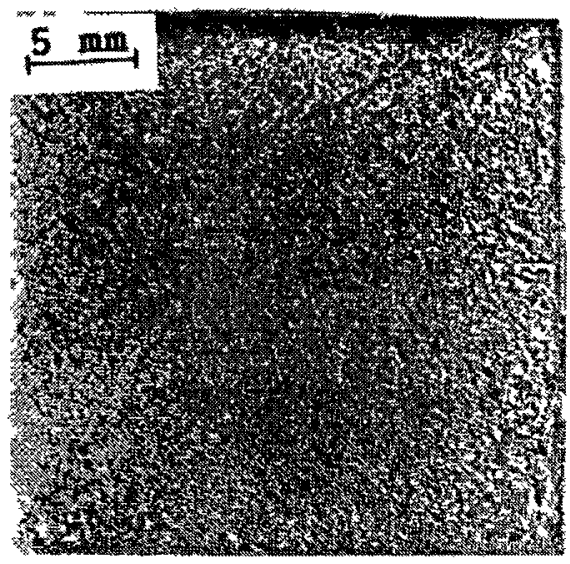

(b)

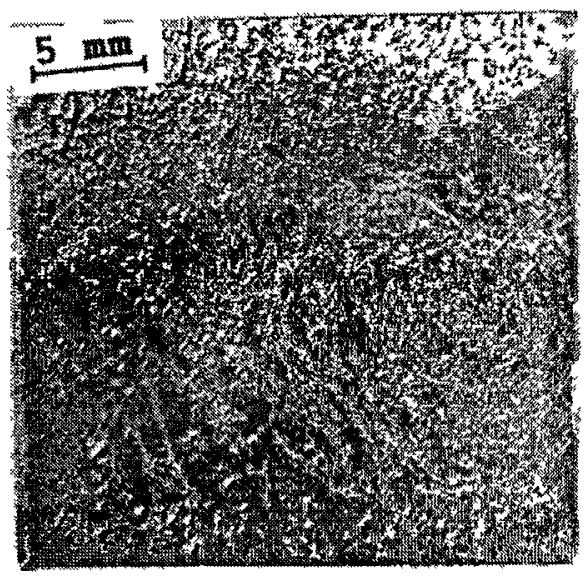

(d)

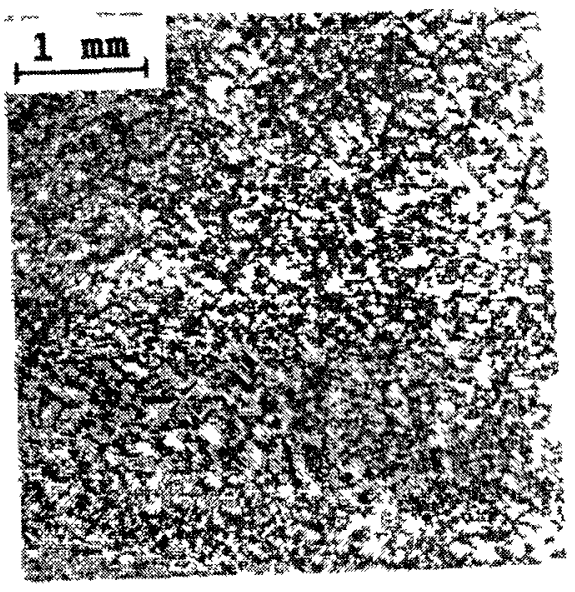

Figure 6.6: Fracture surfaces of the specimens tested at (a) $30^{\circ} \mathrm{C}$, (b) $90^{\circ} \mathrm{C}, \quad$ (c) $120^{\circ} \mathrm{C}$ and (d) $150^{\circ} \mathrm{C}$. The fracture surfaces generally consist of interfacial and cohesive failures as shown at higher magnification in (e) for $150^{\circ} \mathrm{C}$ and in (f) for $30^{\circ} \mathrm{C}$, respectively 


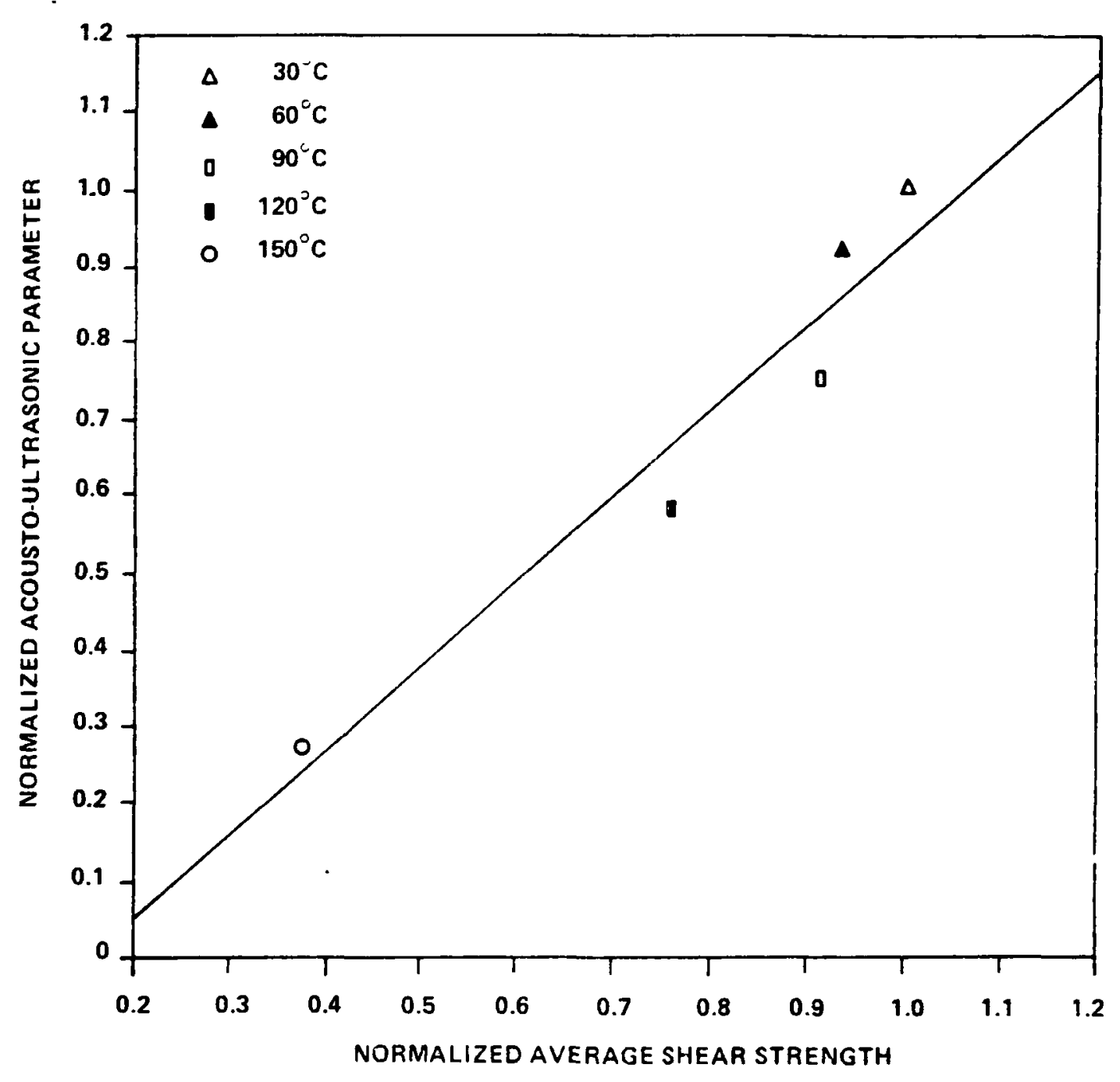

Figure 6.7: Correlation of the acousto-ultrasonic parameter with the average shear strength at different temperatures. The solid line represents the best fit to the data points using the least squares method 
AVERAGE SHEAR STRENGTH (MPa)

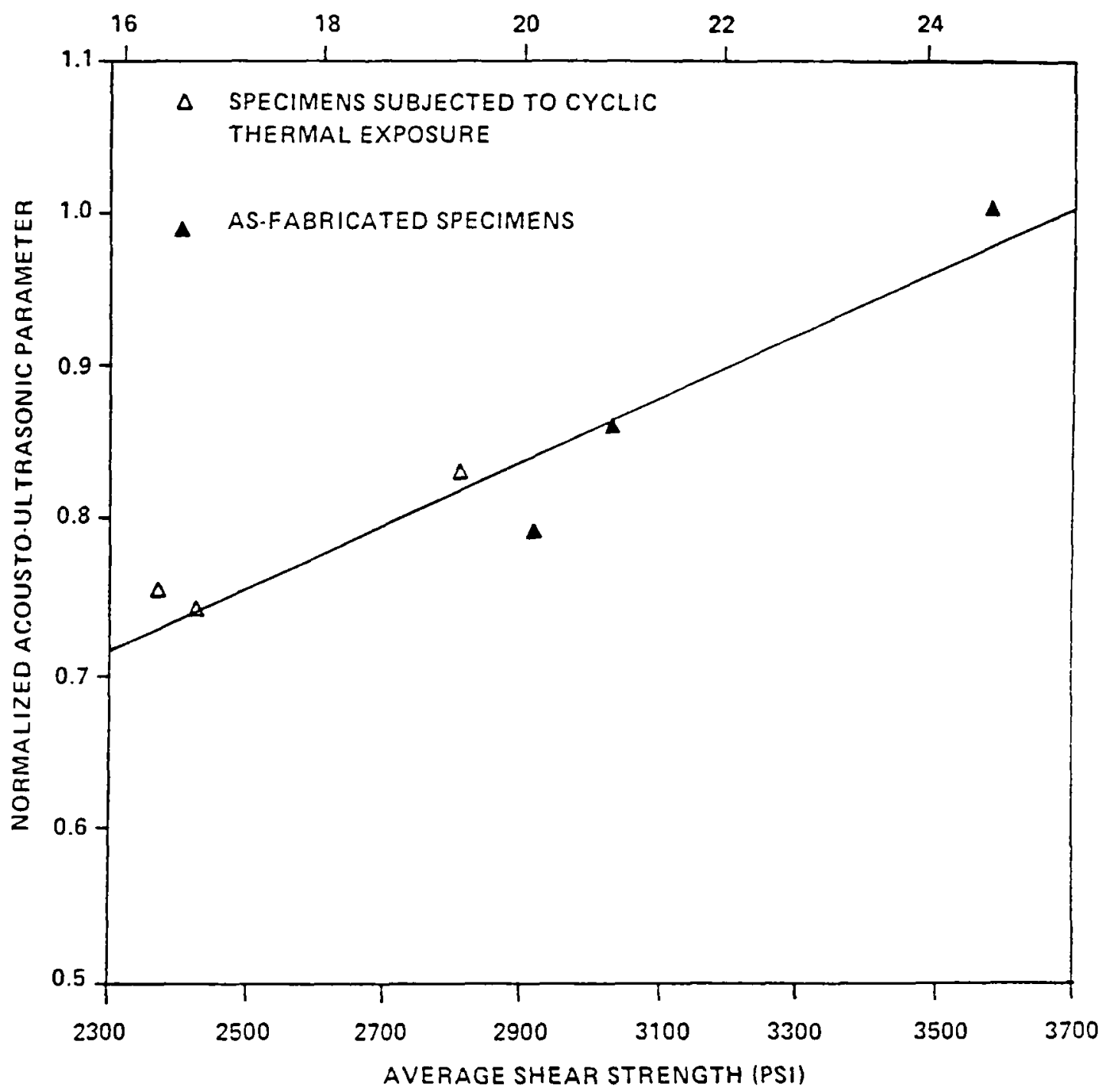

Figure 6.8: Correlation of the acousto-ultrasonic parameter with average shear strength of two groups of specimens; as-fabricated specimens and specimens subjected to cyclic thermal exposure. The solid line indicates the best fit to the data points using the least squares method. 


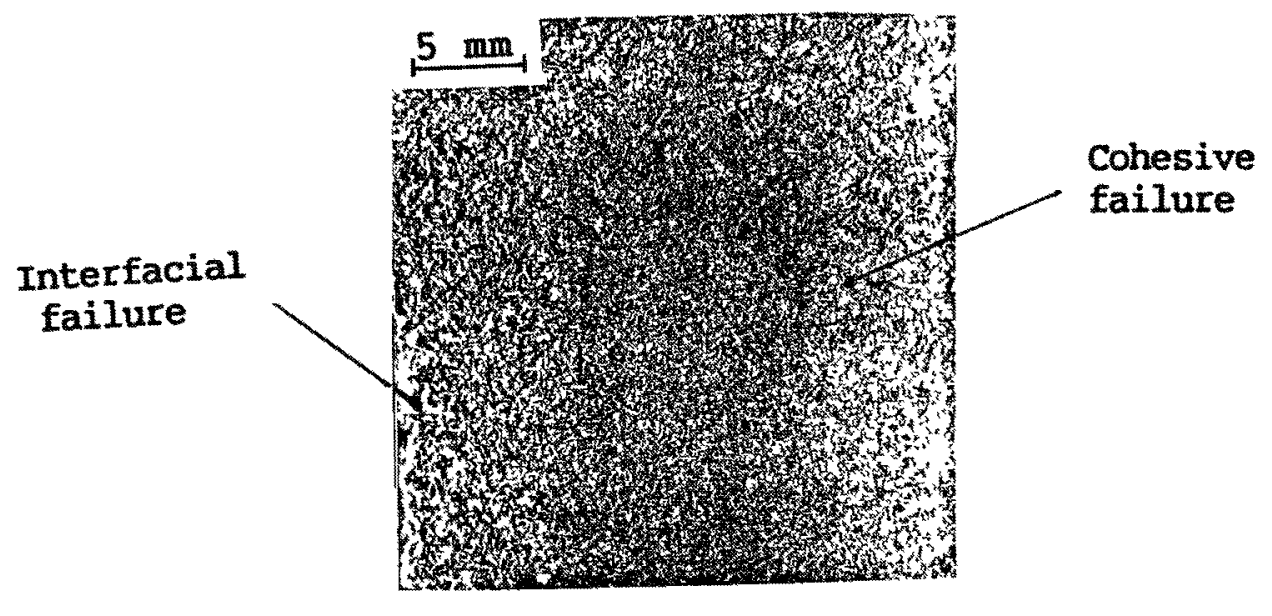

(a)

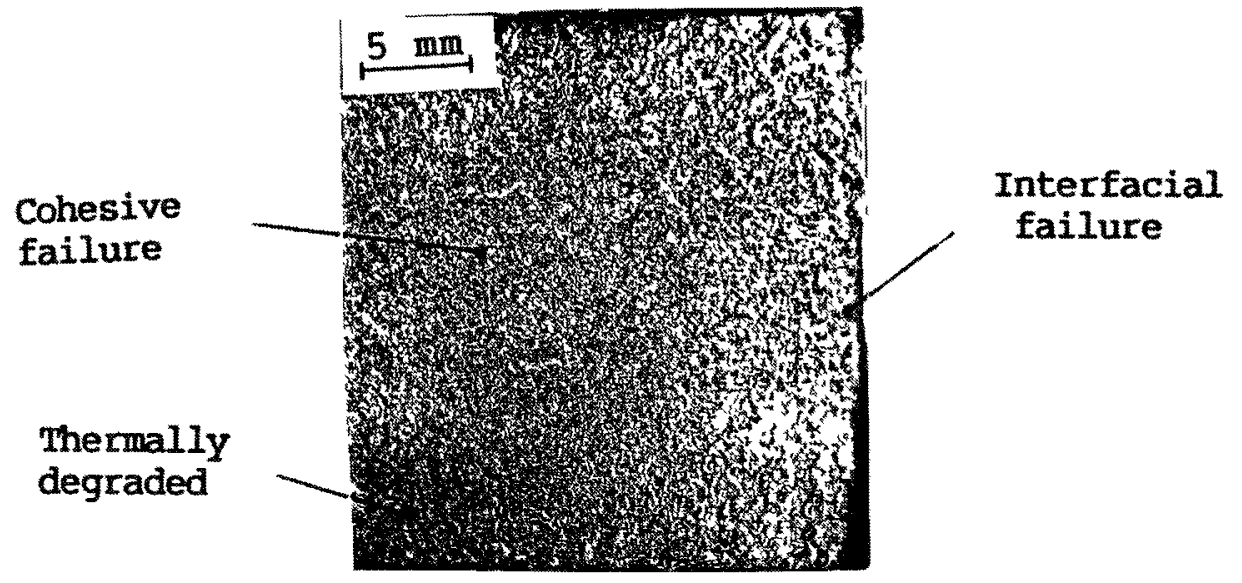

(b)

Figure 6.9: Typical fracture surfaces of (a) an asfabricated specimen as compared with (b) a specimen subjected to cyclic thermal exposure. In both cases, specimens failed predominantly in the cohesive mode but the specimen repeatedly subjected to high temperature showed increased evidence of interfacial failure 


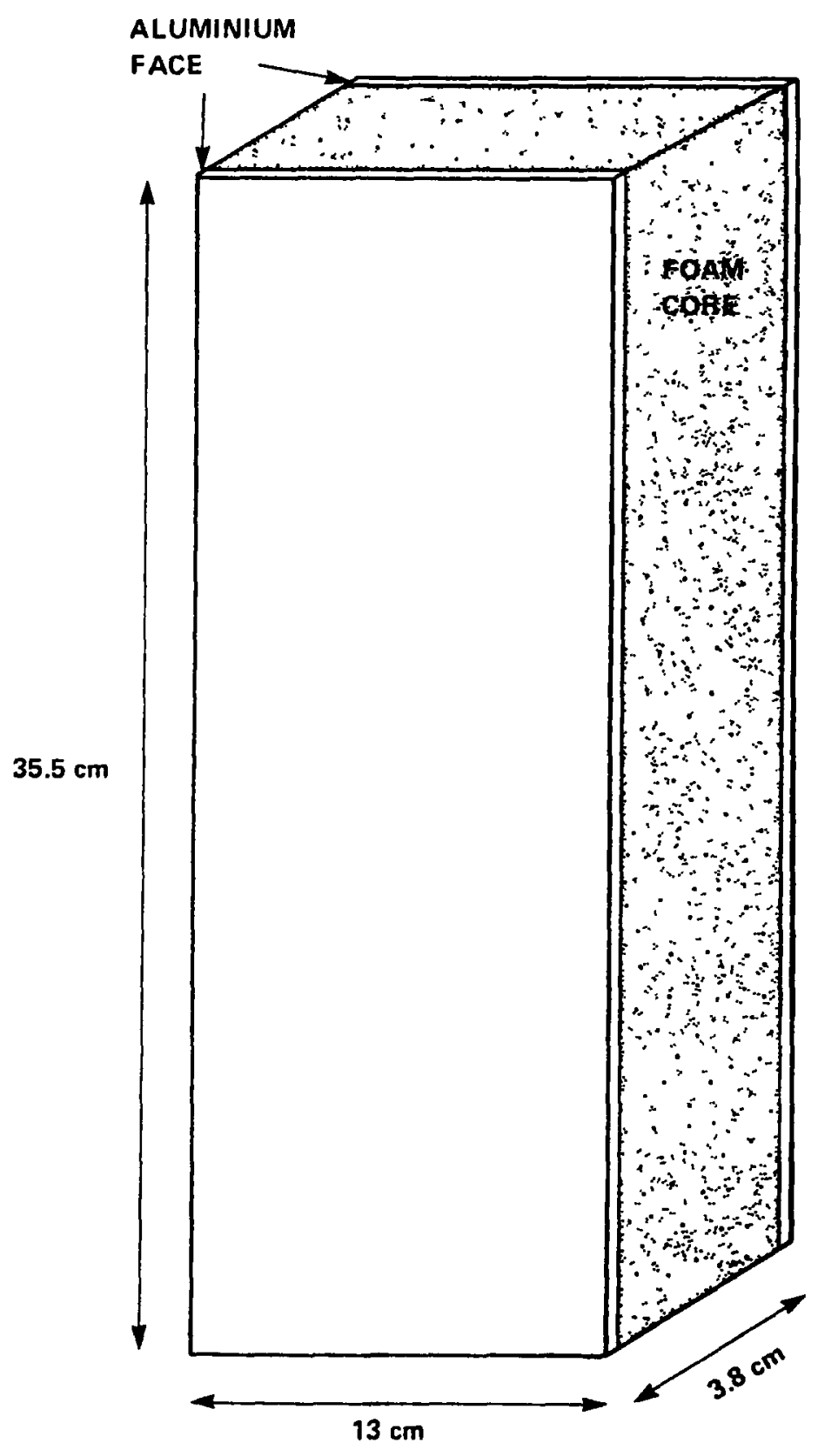

Figure 7.1 : A foam core aluminium test specimen 


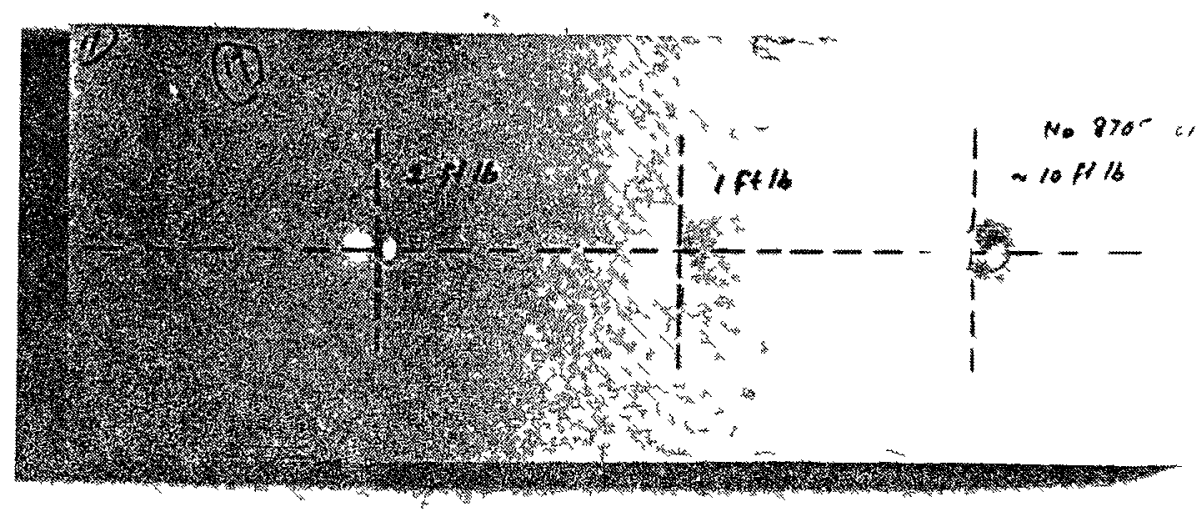

$1.25 \mathrm{~cm}$ dlameter head

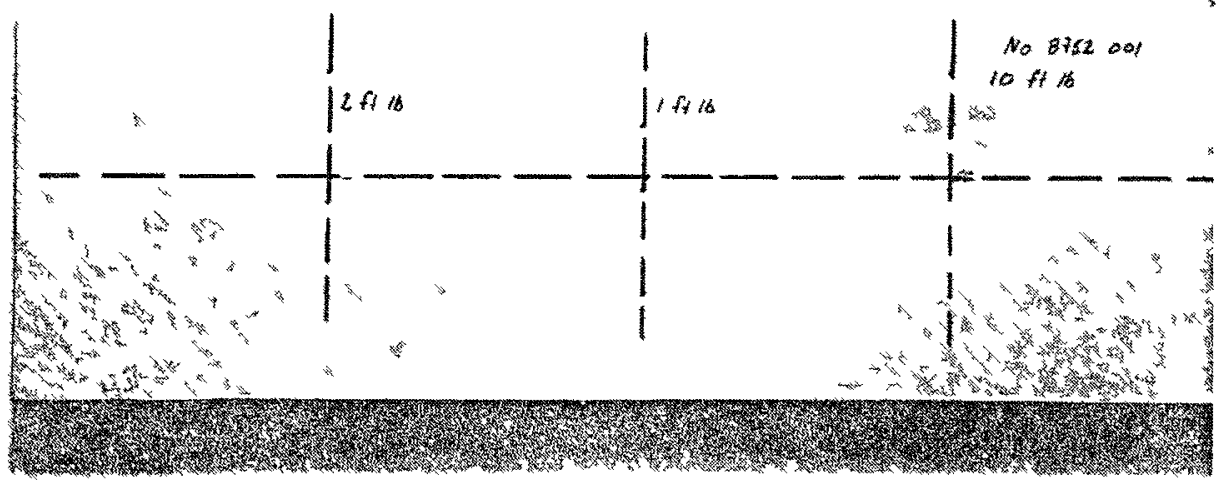

$22.5 \mathrm{~cm}$ drameter indentor

Figure 7.2 : Photographs of test panels after impact at three energy levels using two different impact heads 


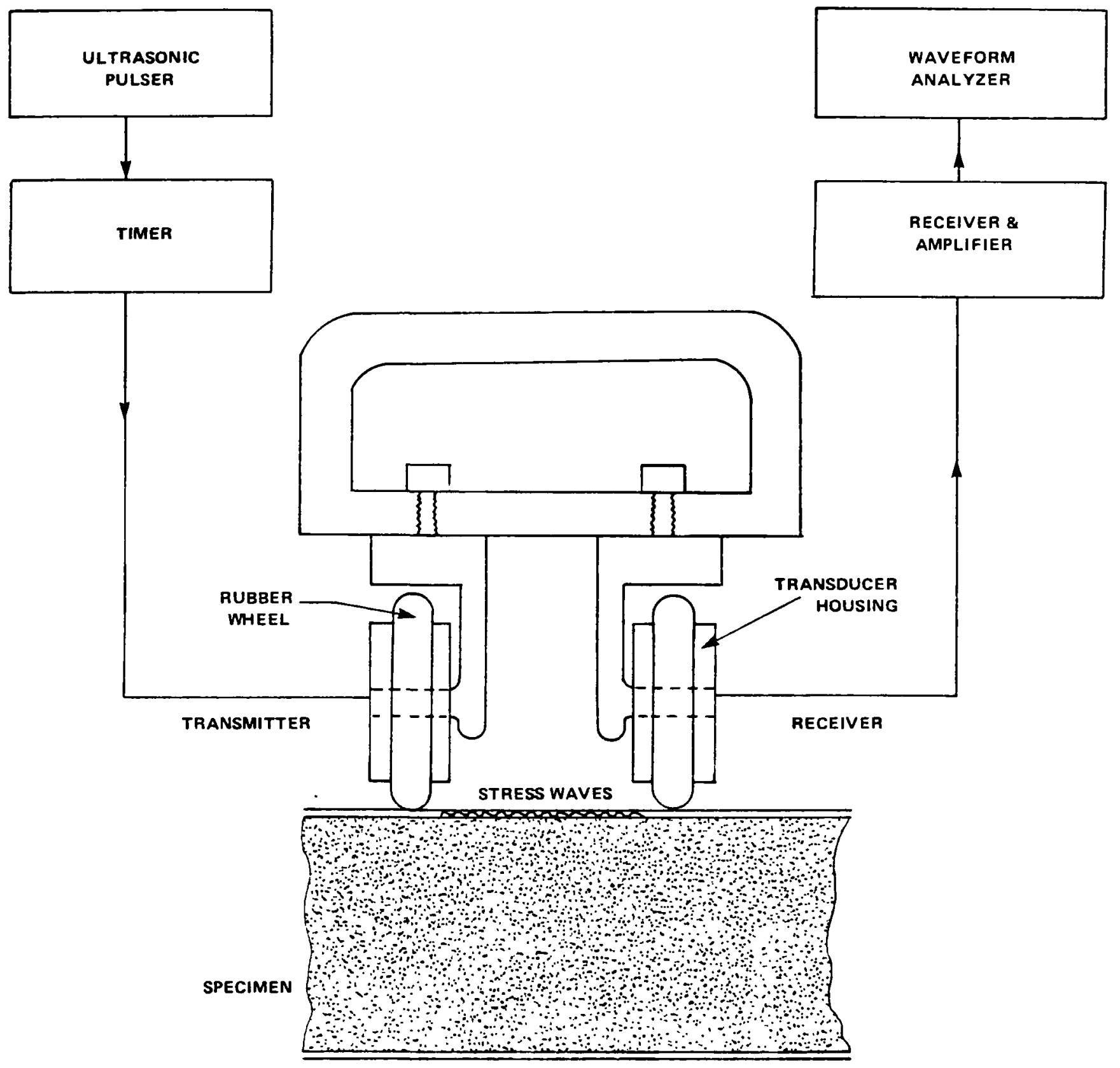

Figure 7.3 : A schematic diagram of the acousto-ultrasonic test set-up for evaluating foam core aluminium panels 


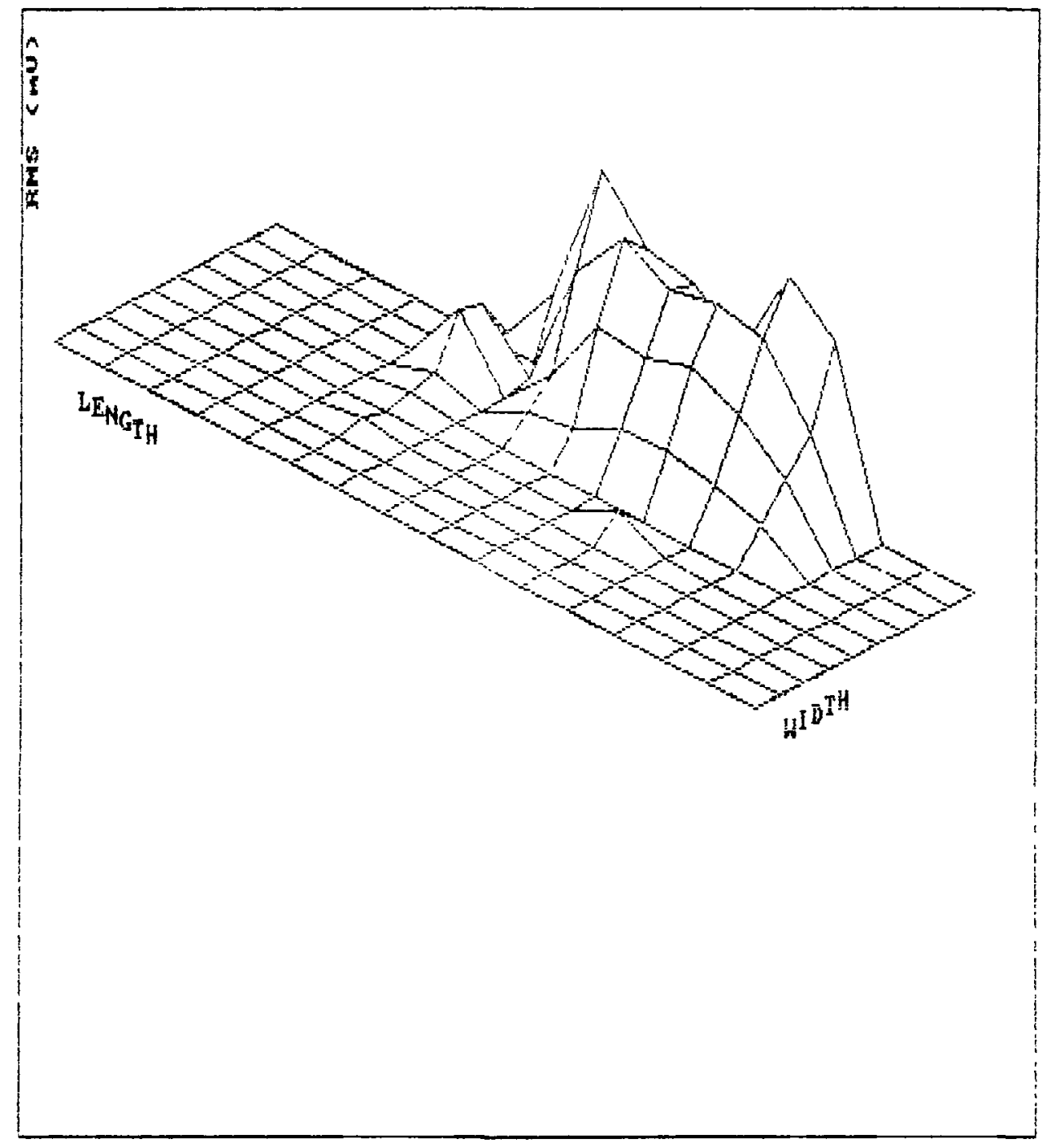
Figure 7.4 : The acousto-ultrasonic test results for the
panel Fo/Al-2 containing fatigue damage 


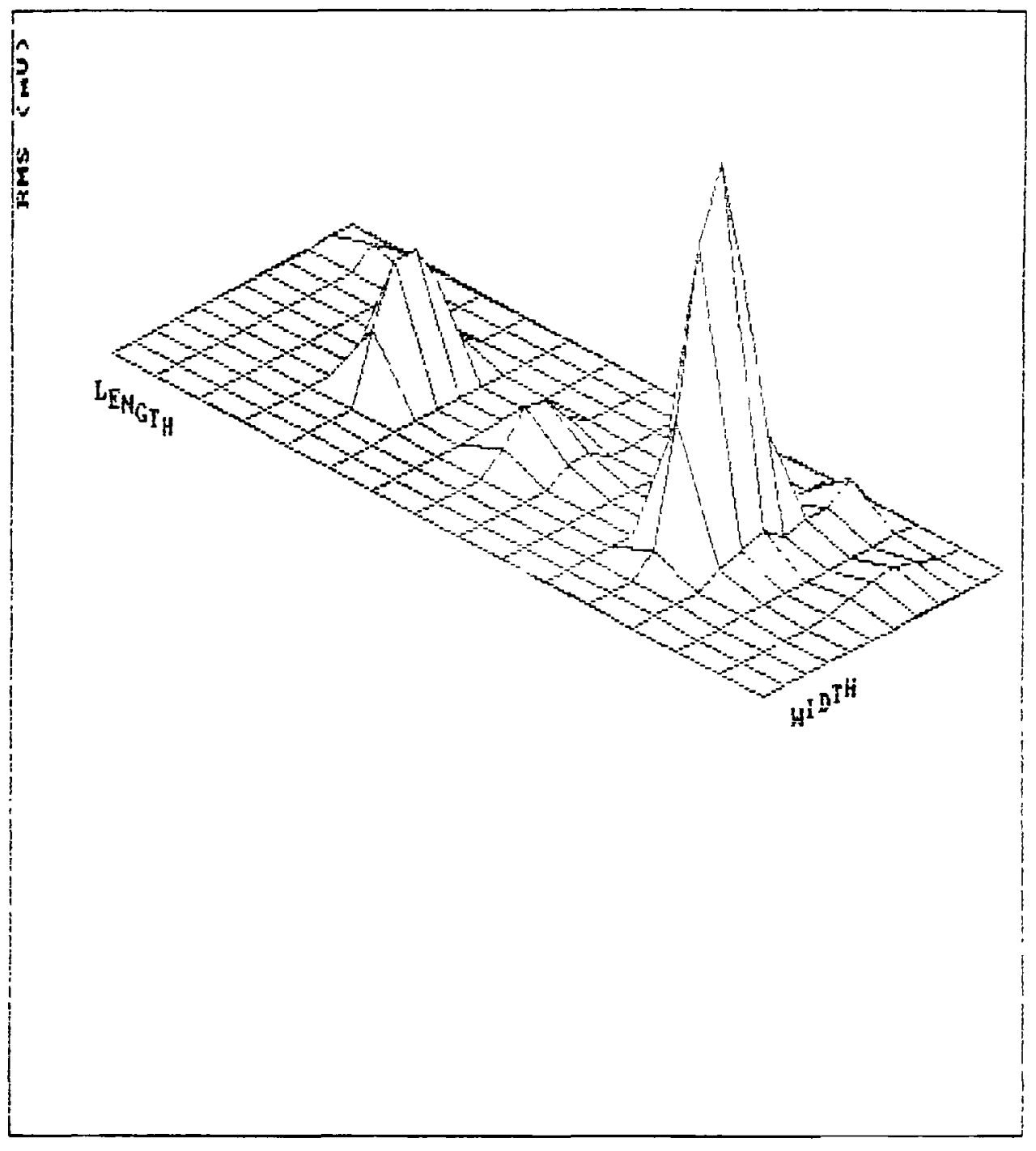

Figure 7.5 : The acousto-ultrasonic test results for the panel Fo/Al-3 containing three visible impact damage sites introduced at $1.5,2.9$ and 14 joules using a $12.5 \mathrm{~mm}$ diameter semispherical impact head 


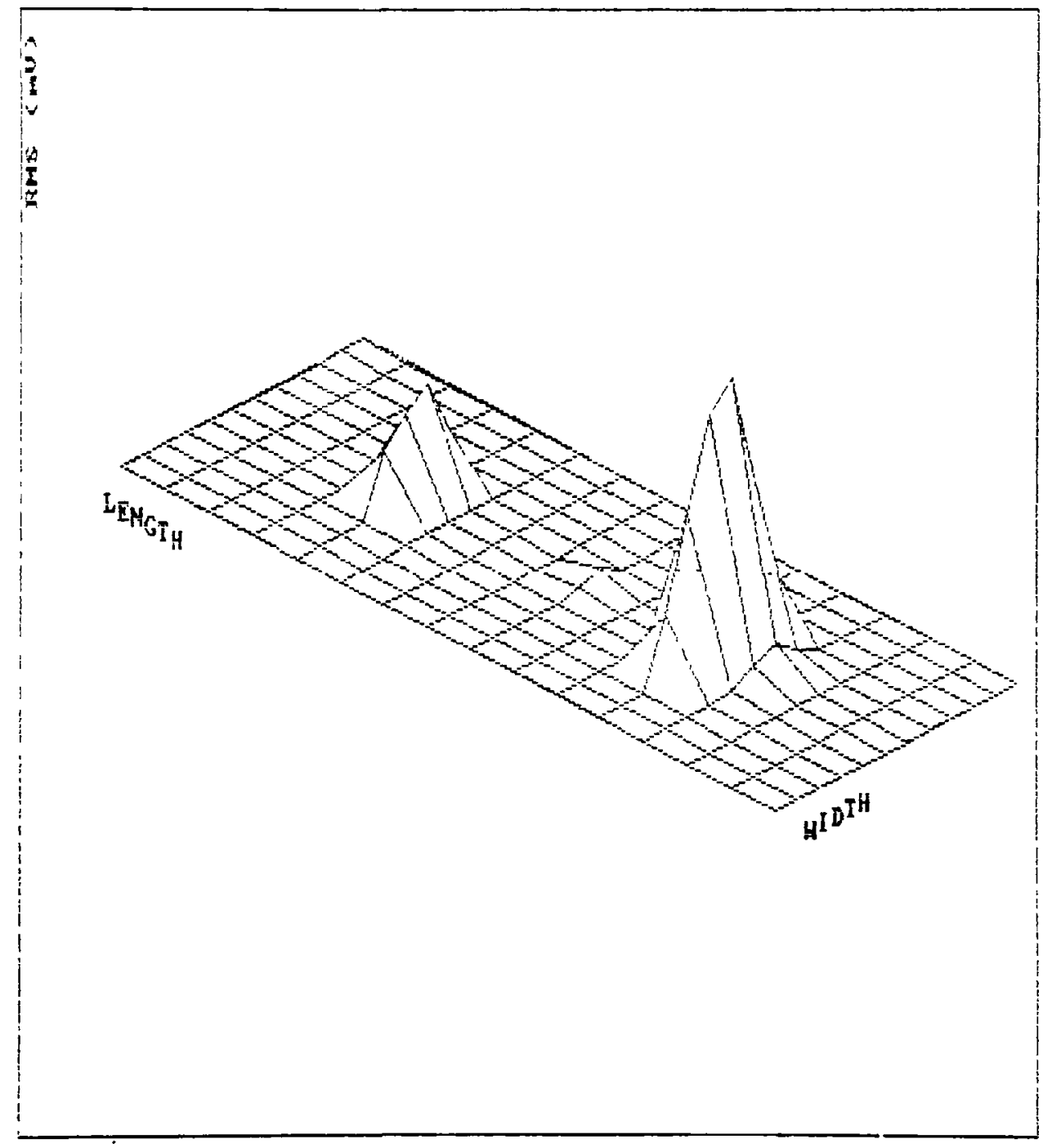

Figure 7.6 : The acousto-ultrasonic test results for the panel $\mathrm{Fo} / \mathrm{Al}-4$ containing two invisible and one visible impact damage sites introduced at $1.4,2.8$ and 13.9 joules using a nearly flat indentor 


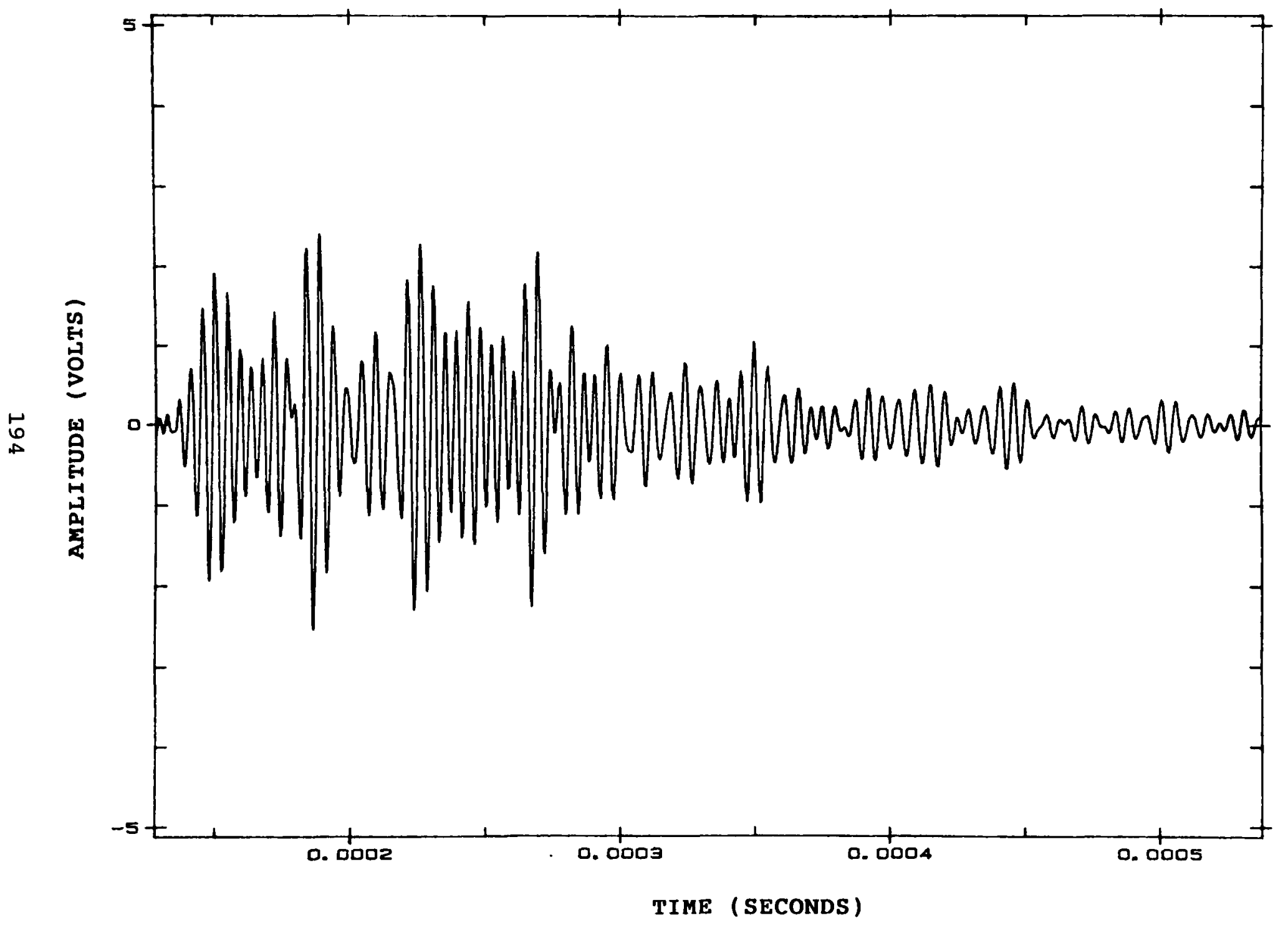

Figure 7.7 : A typical acousto-ultrasonic waveform for the as-received (AR) pane] 


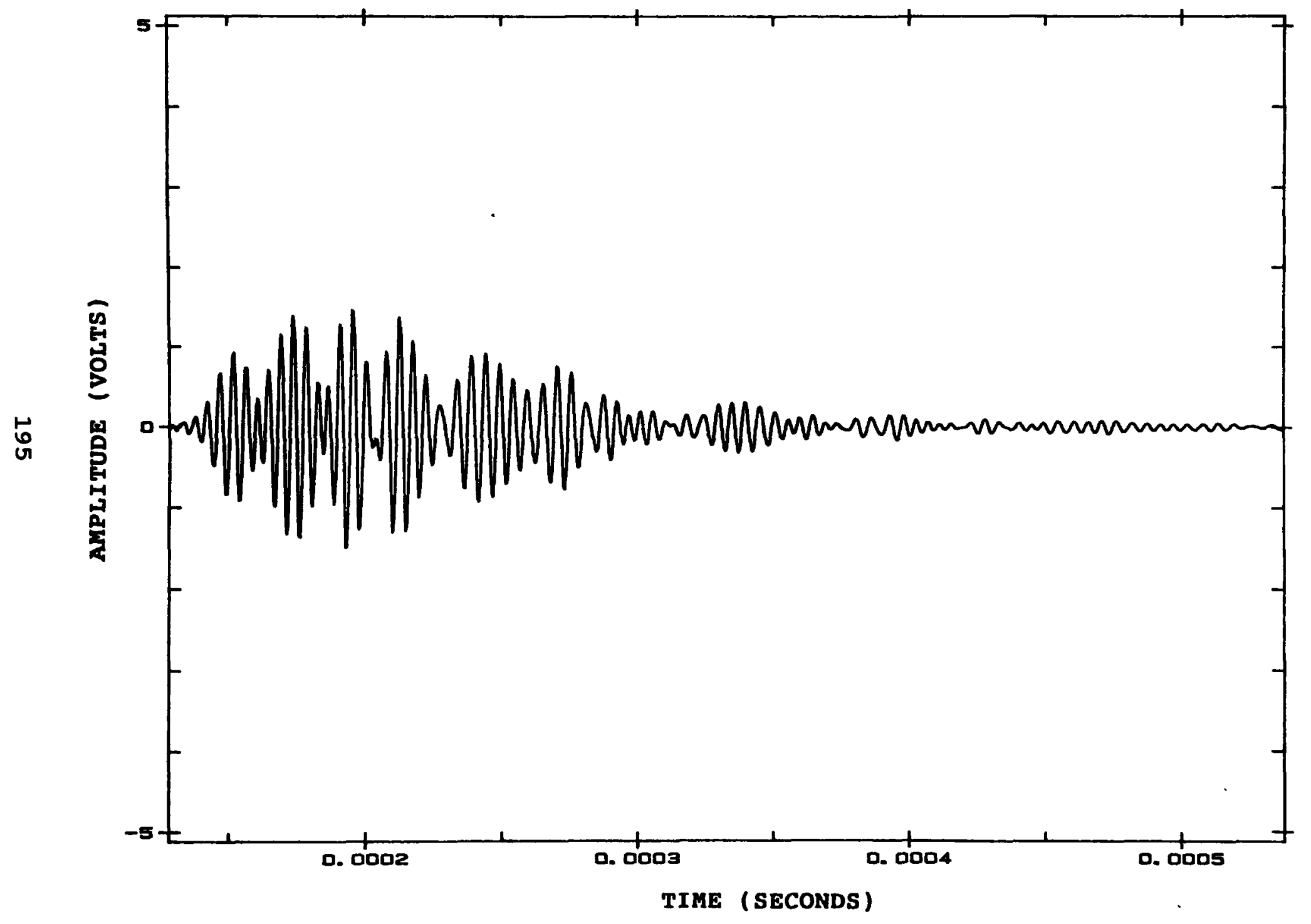

Figure 7.8 : A typical acousto-ultrasonic waveform for the as-received panel with priming (ARP) 


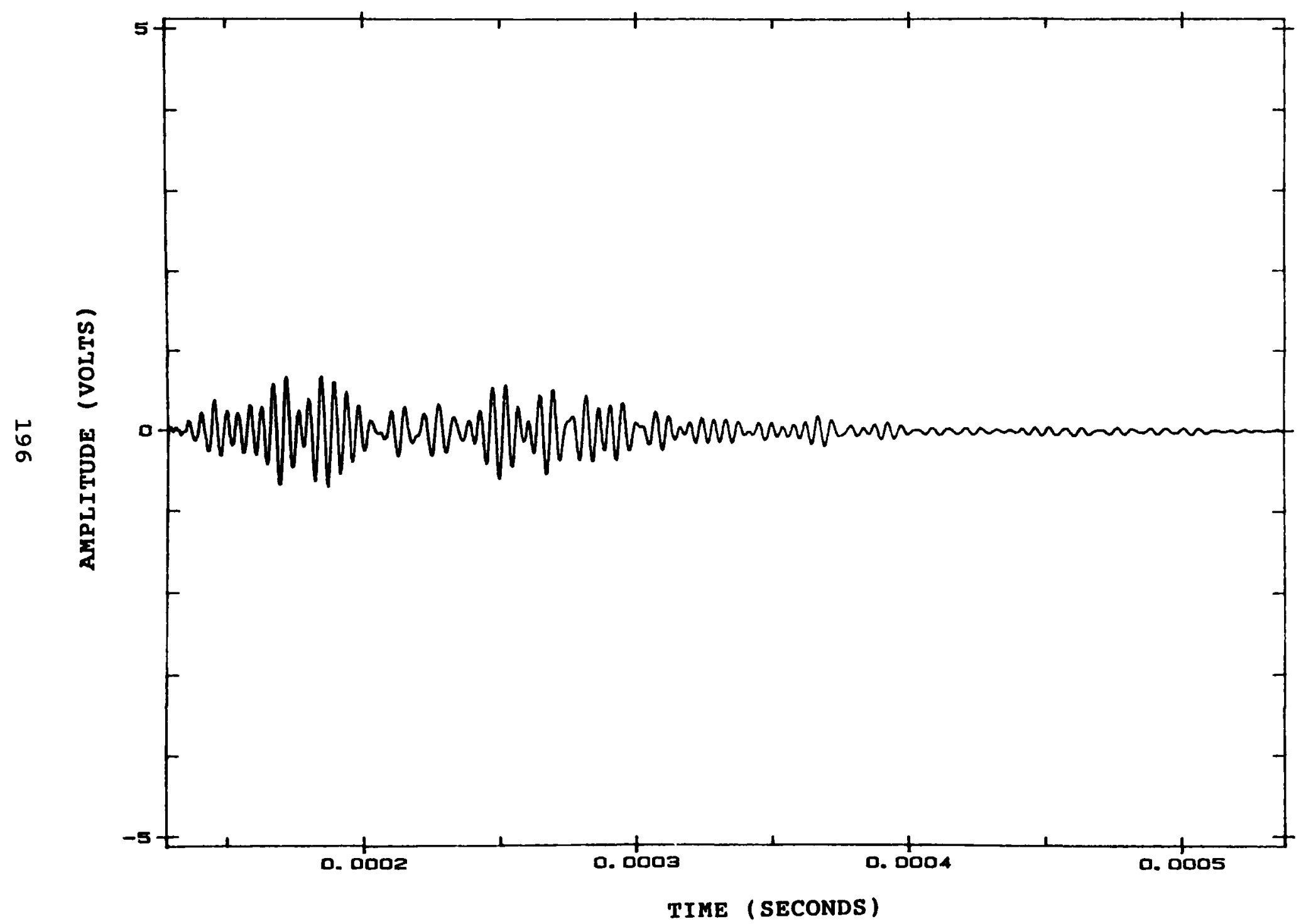

Figure 7.9 : A typical acousto-ultrasonic waveform for the panel with surface preparation technique of NRP (new roller with priming) 


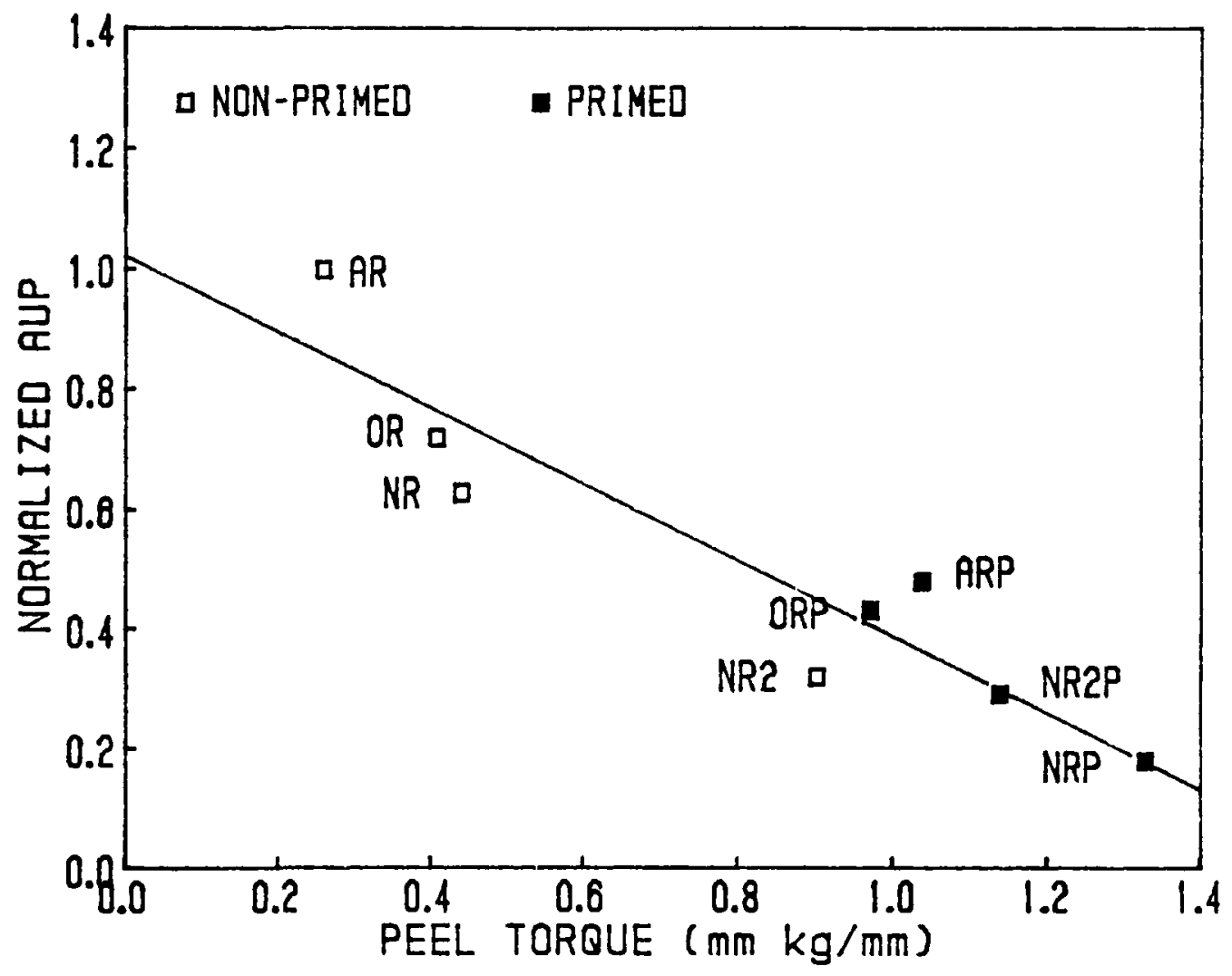

Figure 7.10 : Correlation of acousto-ultrasonic parameter (AUP) with peel torque of foam core aluminium panels. The AUP is normalized relative to the maximum value of AUP in the given data. The solid line represents the best fit to the data points using the least squares method. The specimen code (see Table 7.2) indicates the surface preparation technique. 


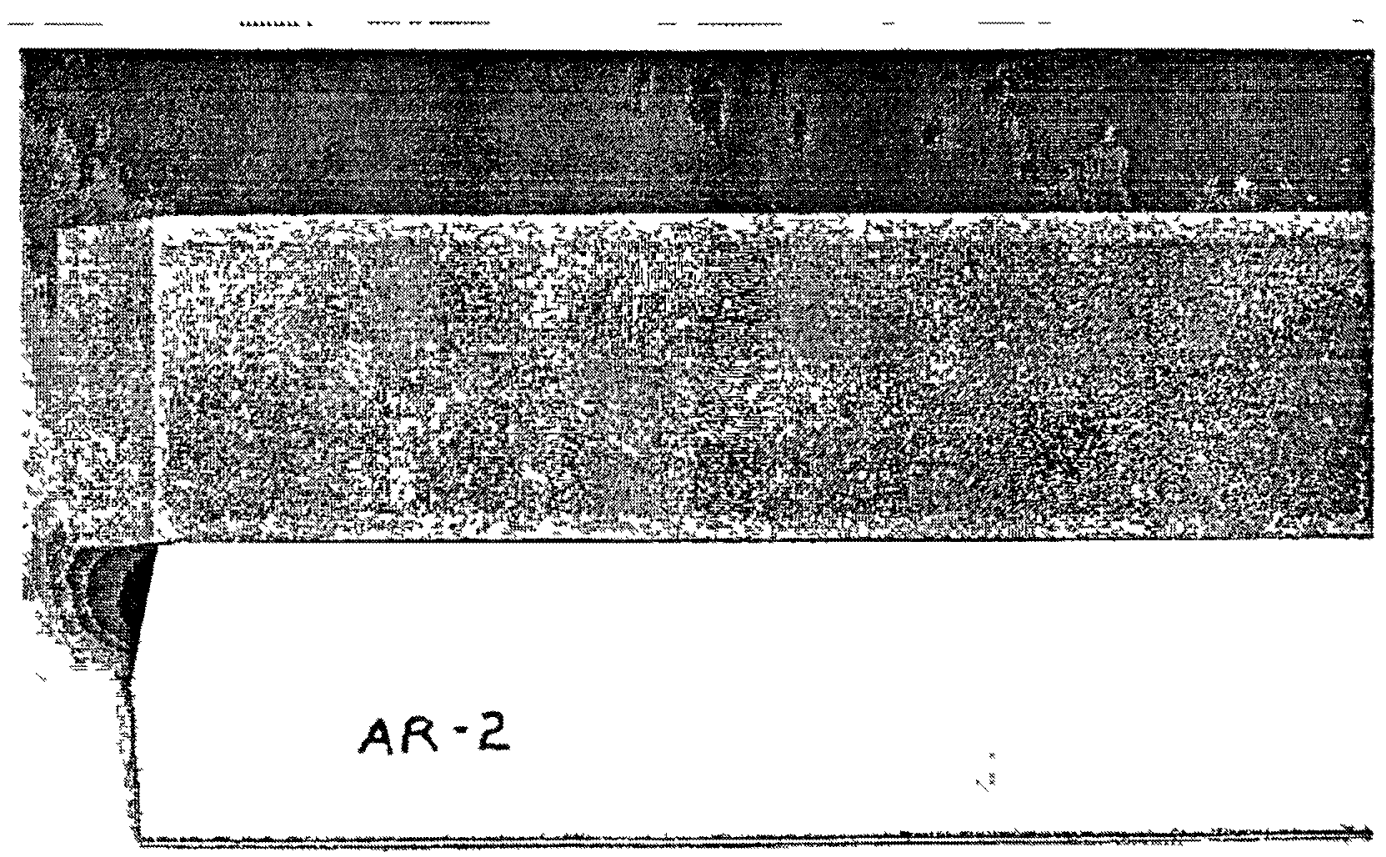

Figure 7.11 : A representative fracture surface for the asreceived (AR) panel. The failure mode was completely by interfacial failure 


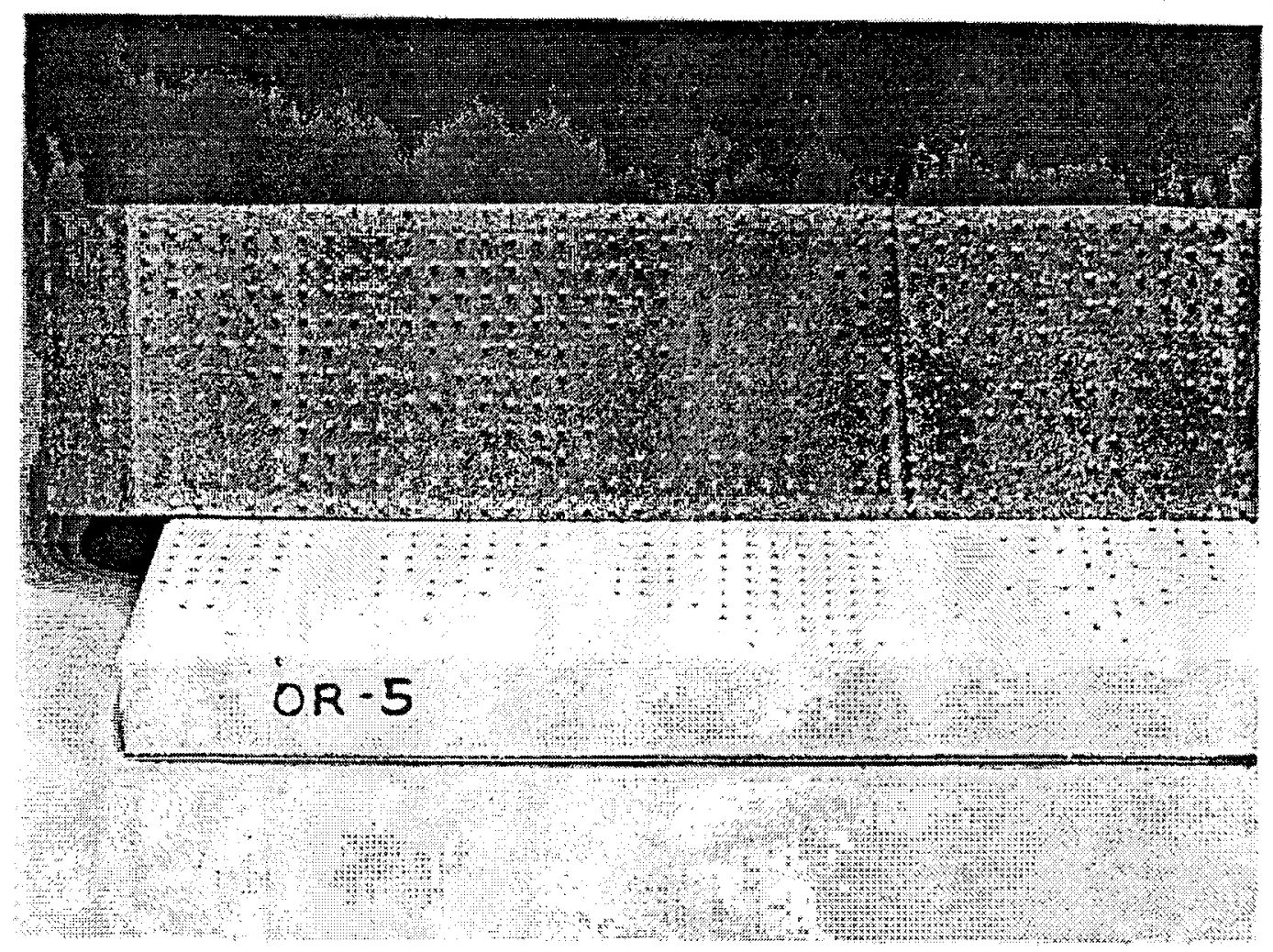

Figure 7.12 : A representative fracture surface for the panel with old roller (OR) surface preparation technique. The dots on the foam core indicate the holes where cohesive failure in the adhesive occurred 


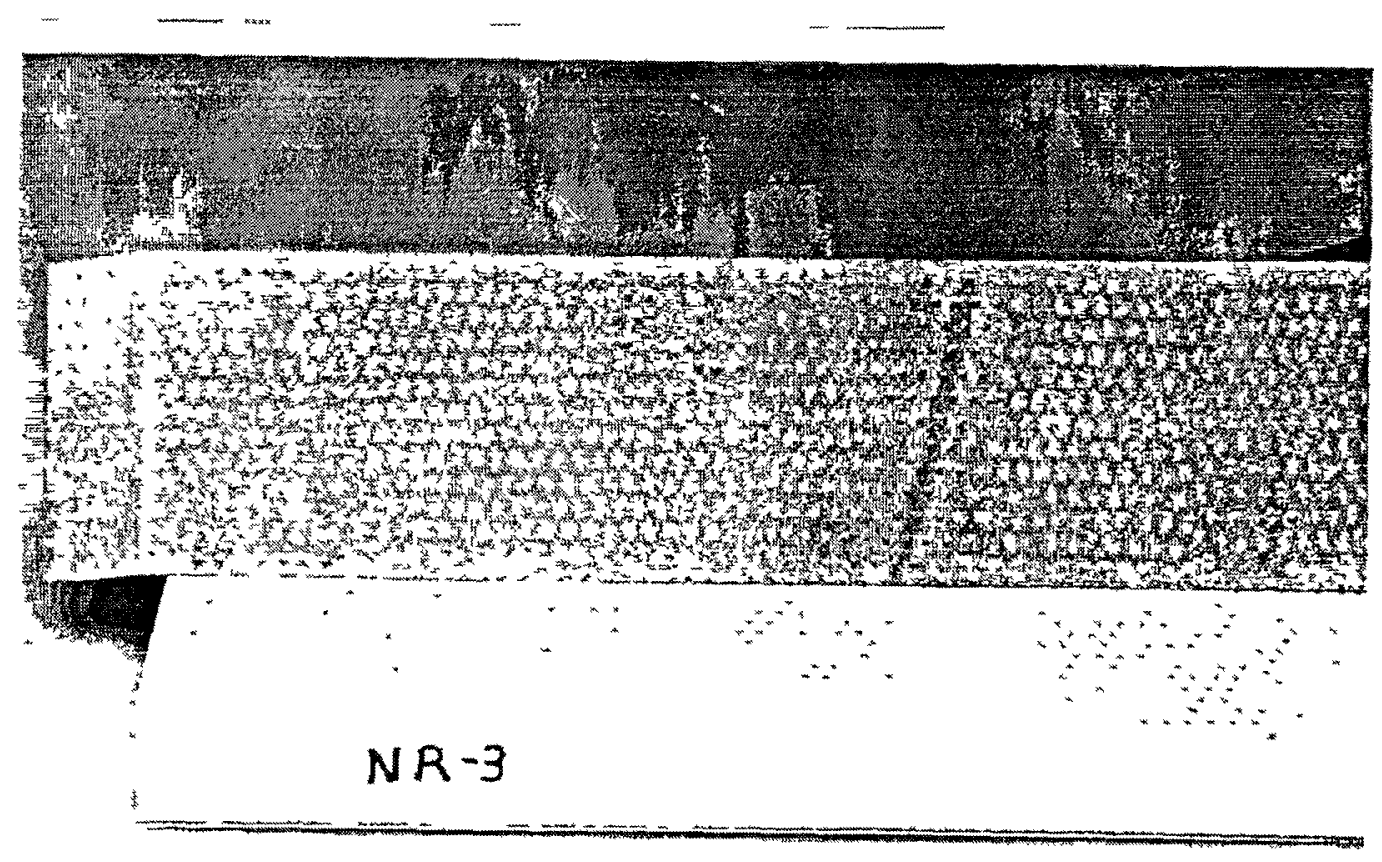

Figure 7.13 : A representative fracture surface for the panel with new roller (NR) surface preparation technique. The dots on the foam core indicate the holes where cohesive failure in the adhesive occurred 


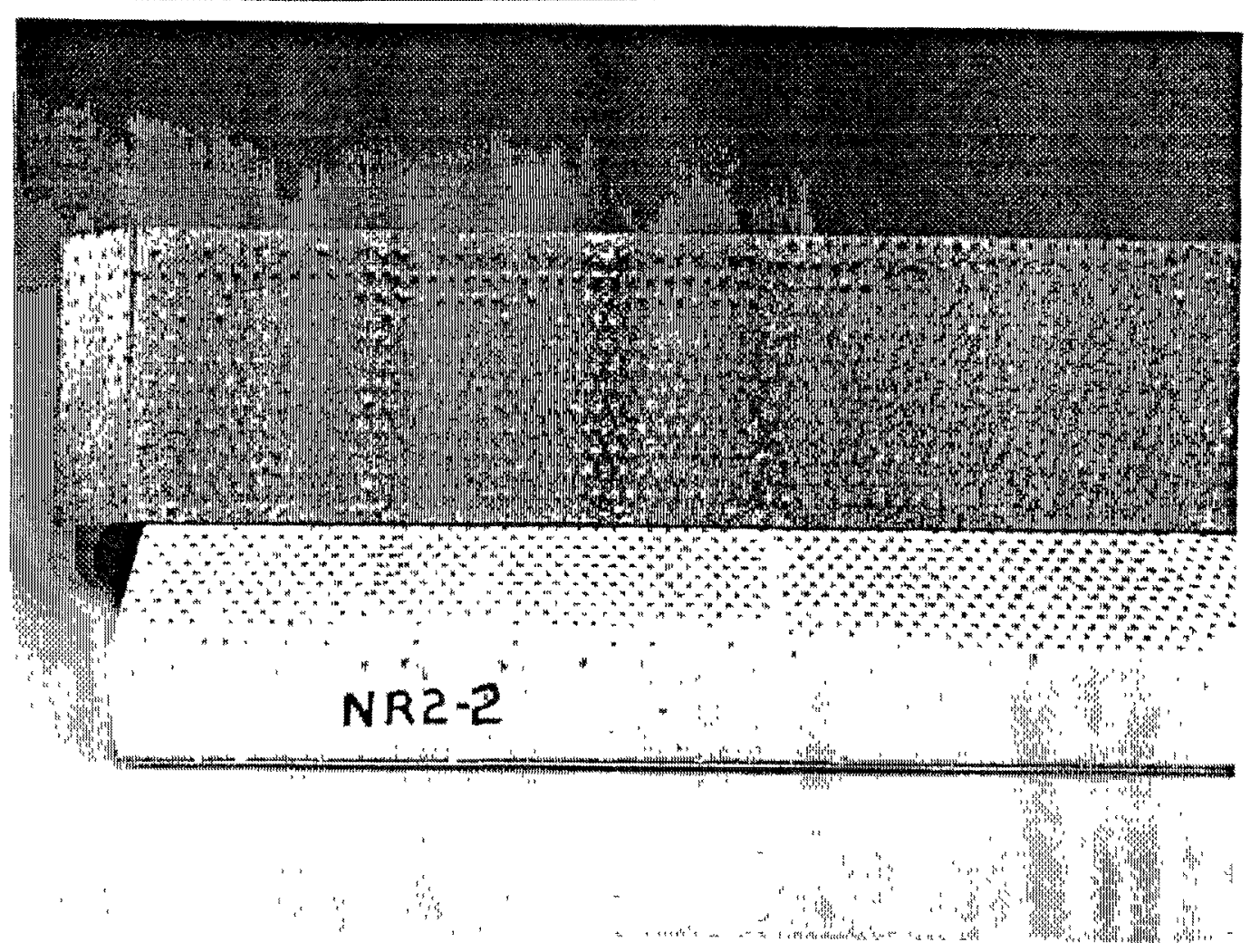

Figure 7.14 : A representative fracture surface for the panel with new roller surface preparation technique and with two layers of adhesive (NR2). The dots on the foam core indicate the holes where cohesive failure in the adhesive occurred 


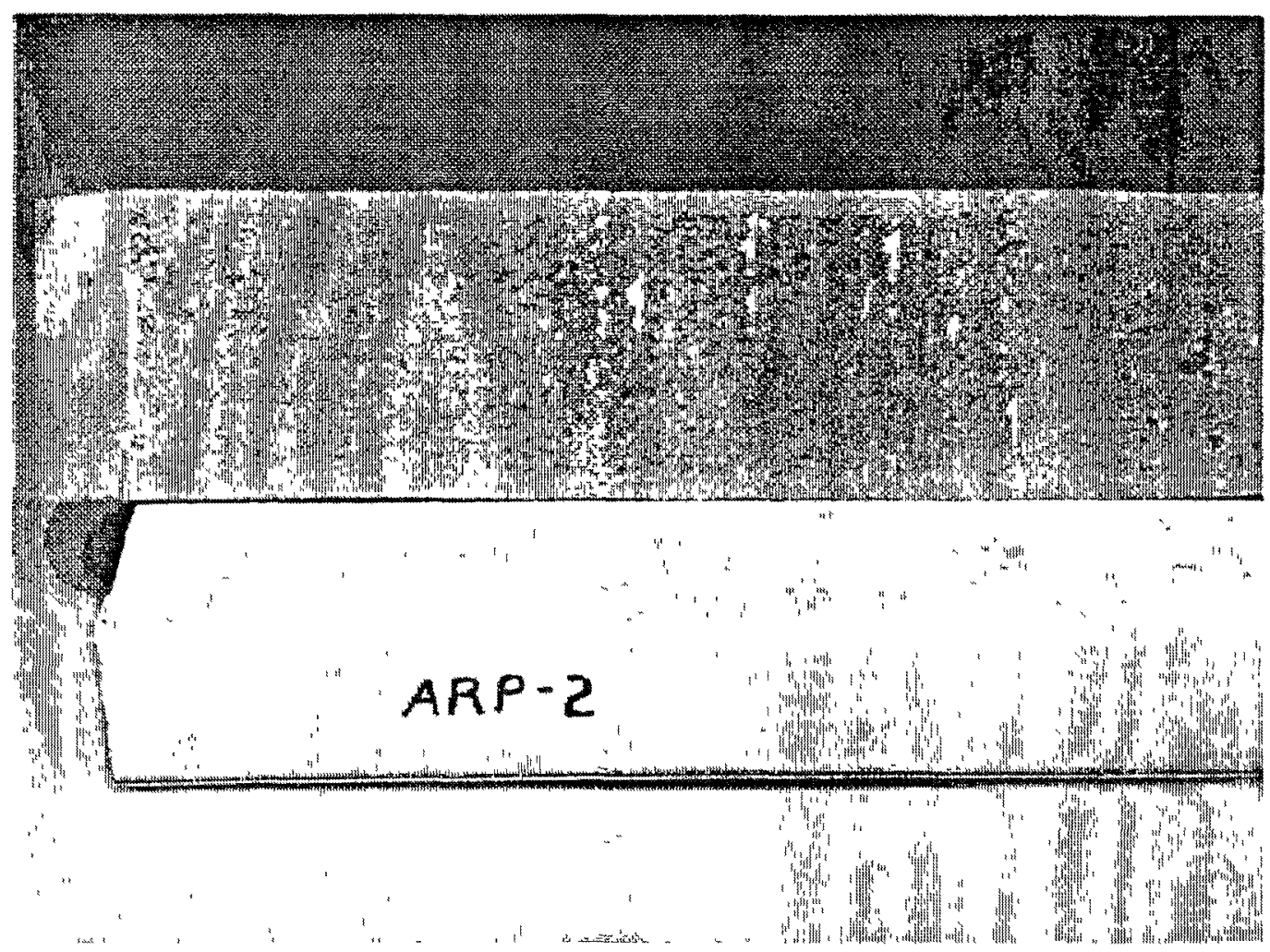

Figure 7.15 : A representative fracture surface for the asreceived panel with priming (ARP). The predominant failure mode was interfacial failure with some cohesive failure in the adhesive and in the foam core 


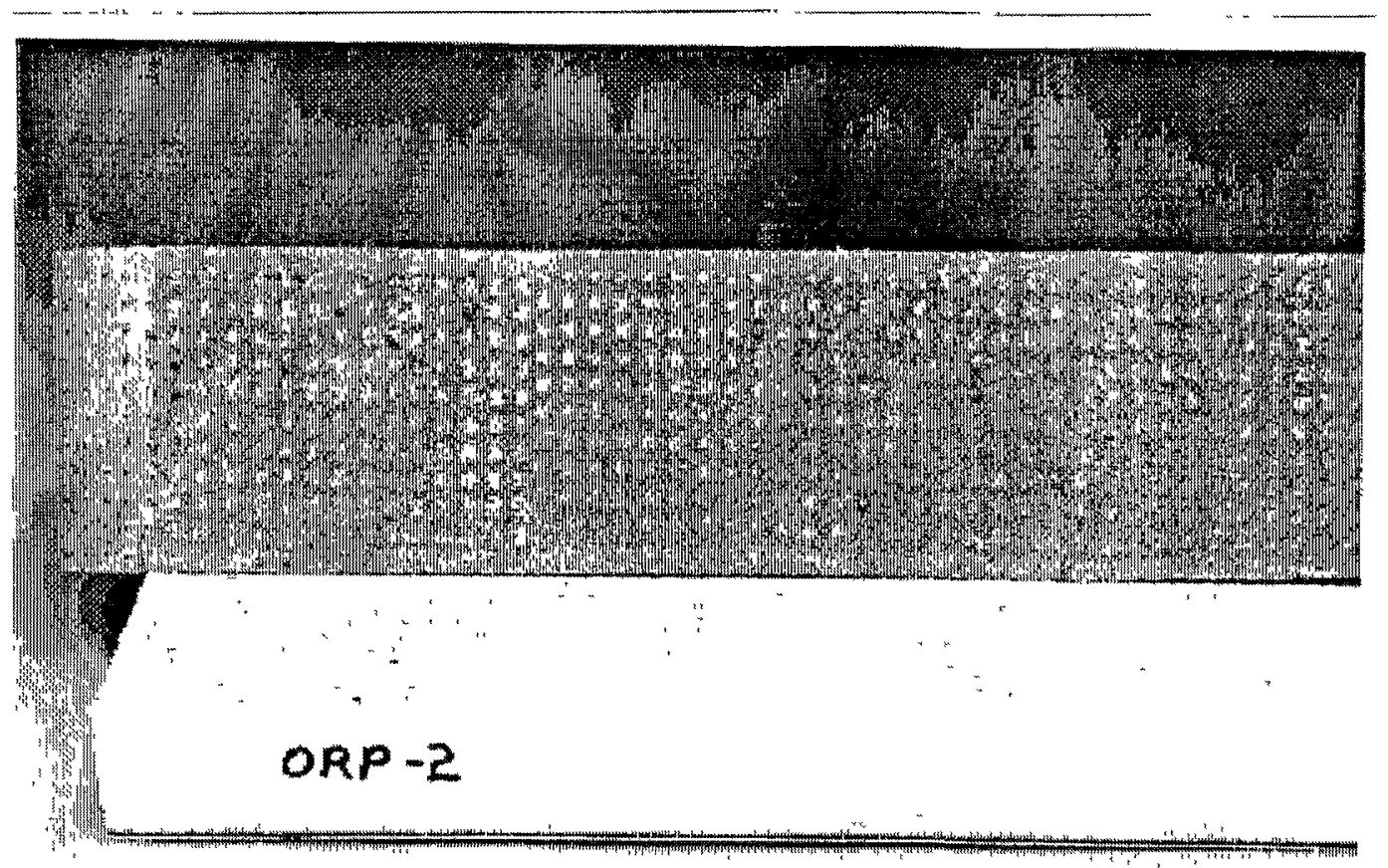

Figure 7.16 : A representative fracture surface for the panel with surface preparation technique of ORP (old roller with priming). The dots on the foam core indicate the holes where cohesive failure in the adhesive occurred 


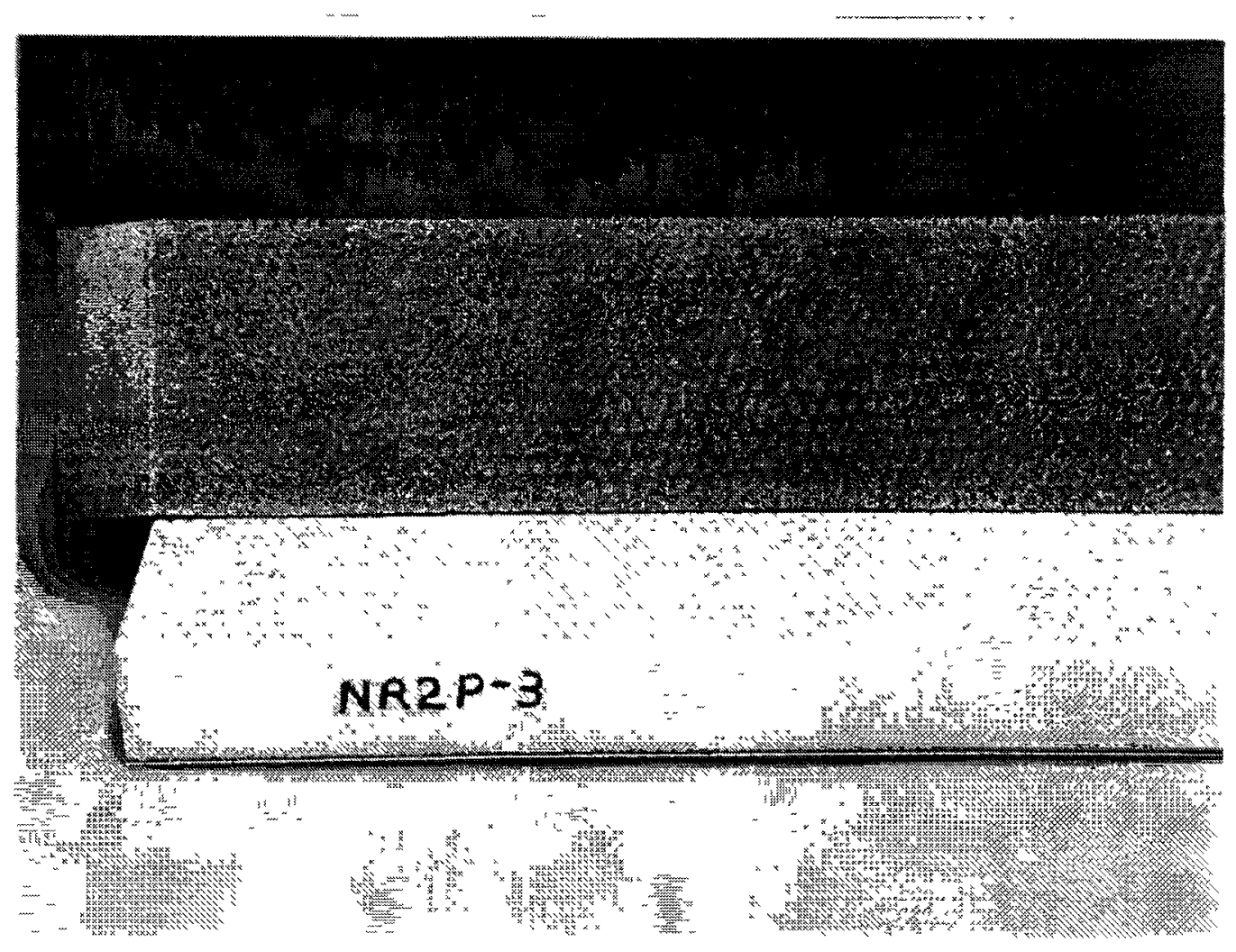

Figure 7.17 : A representative fracture surface for the panel with surface preparation technique of NR2P (new roller with 2 layers of adhesive and with priming). The dots on the foam core indicate the holes where cohesive failure in the adhesive occurred 


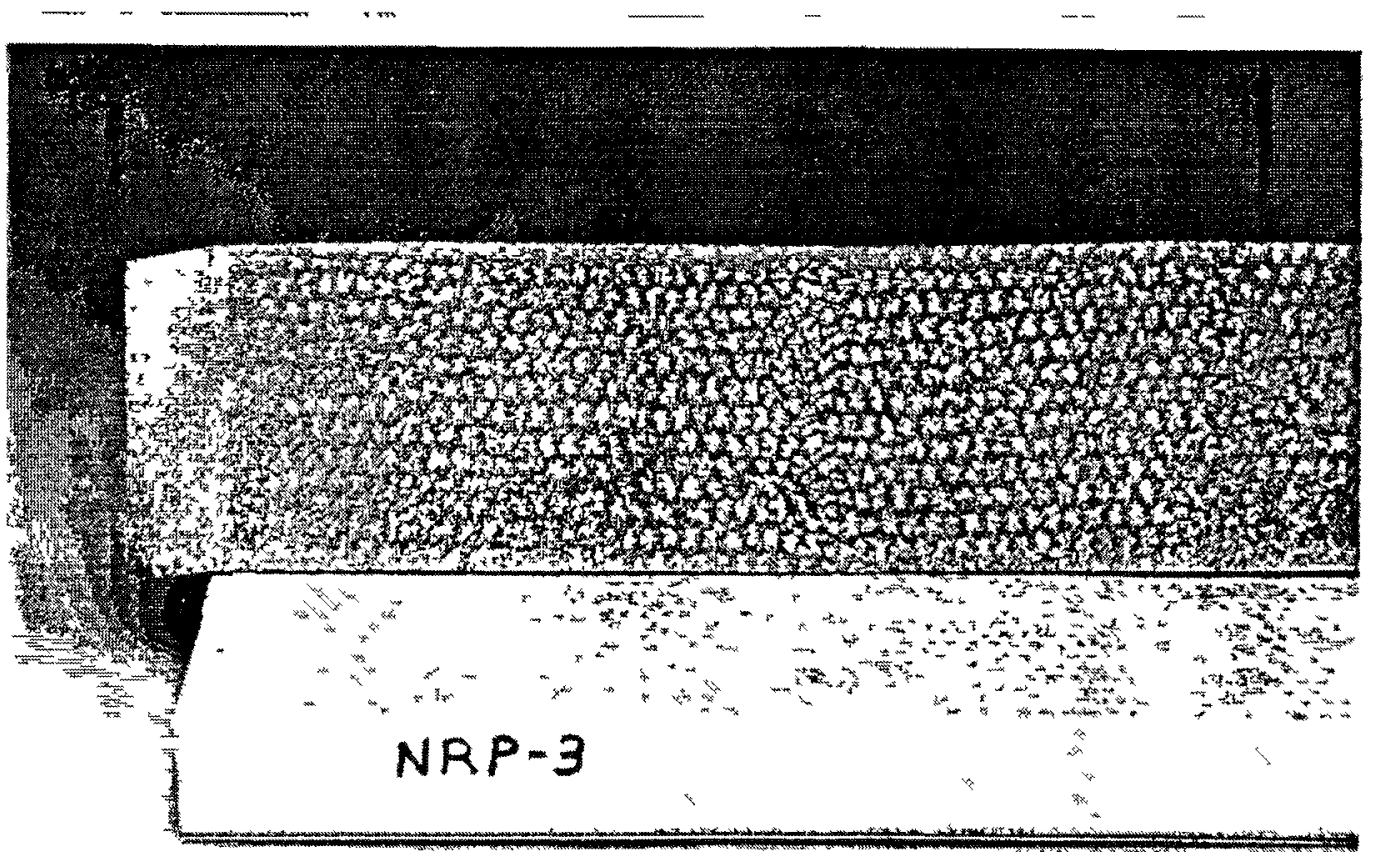

Figure 7.18 : A representative fracture surface for the panel with surface preparation technique of NRP (new roller with priming). The failure mode was predominantly cohesive failure in the adhesive 


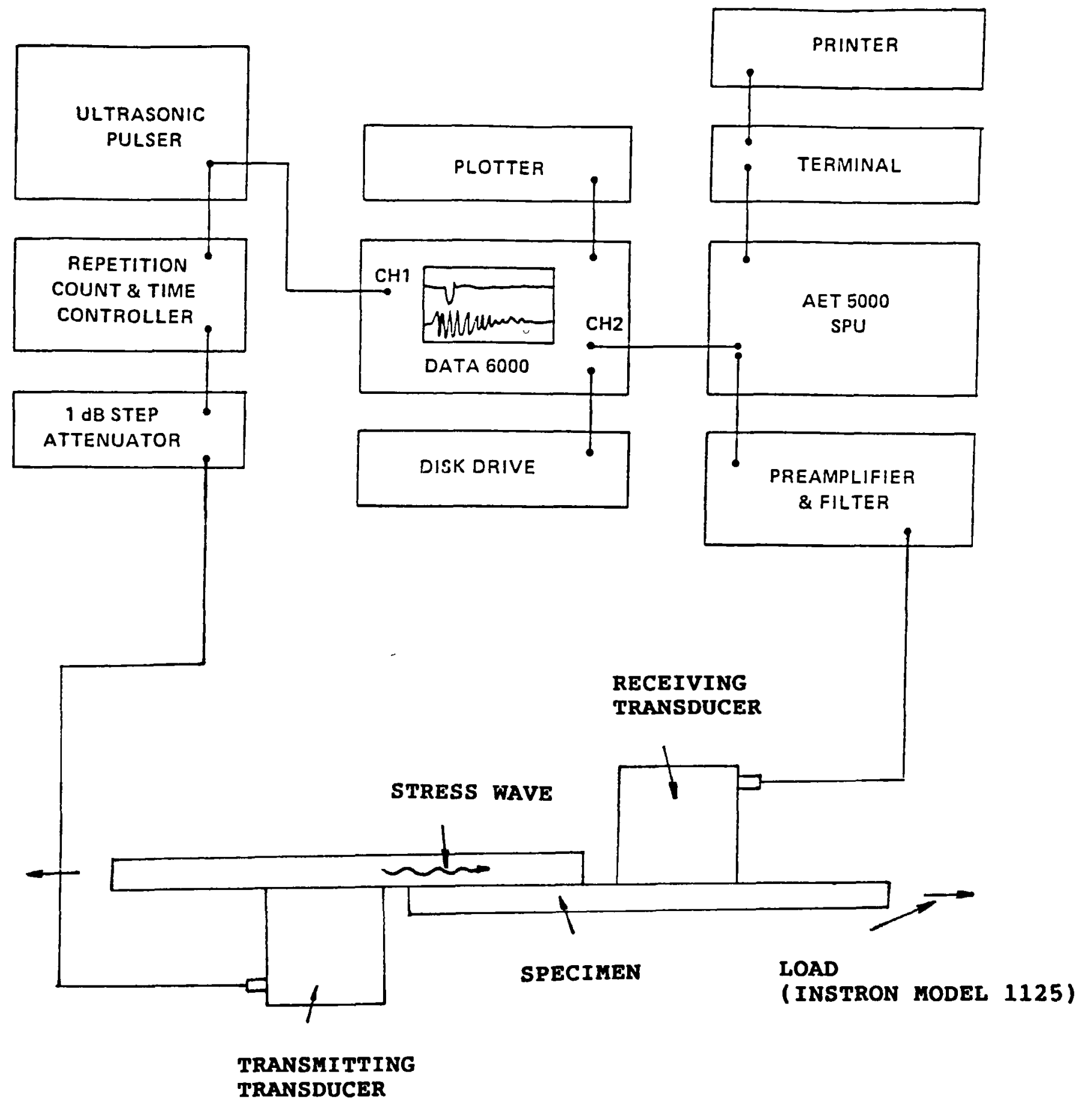

Figure 8.1: A schematic diagram of the acousto-ultrasonic monitoring a joint specimen under tensile loading 


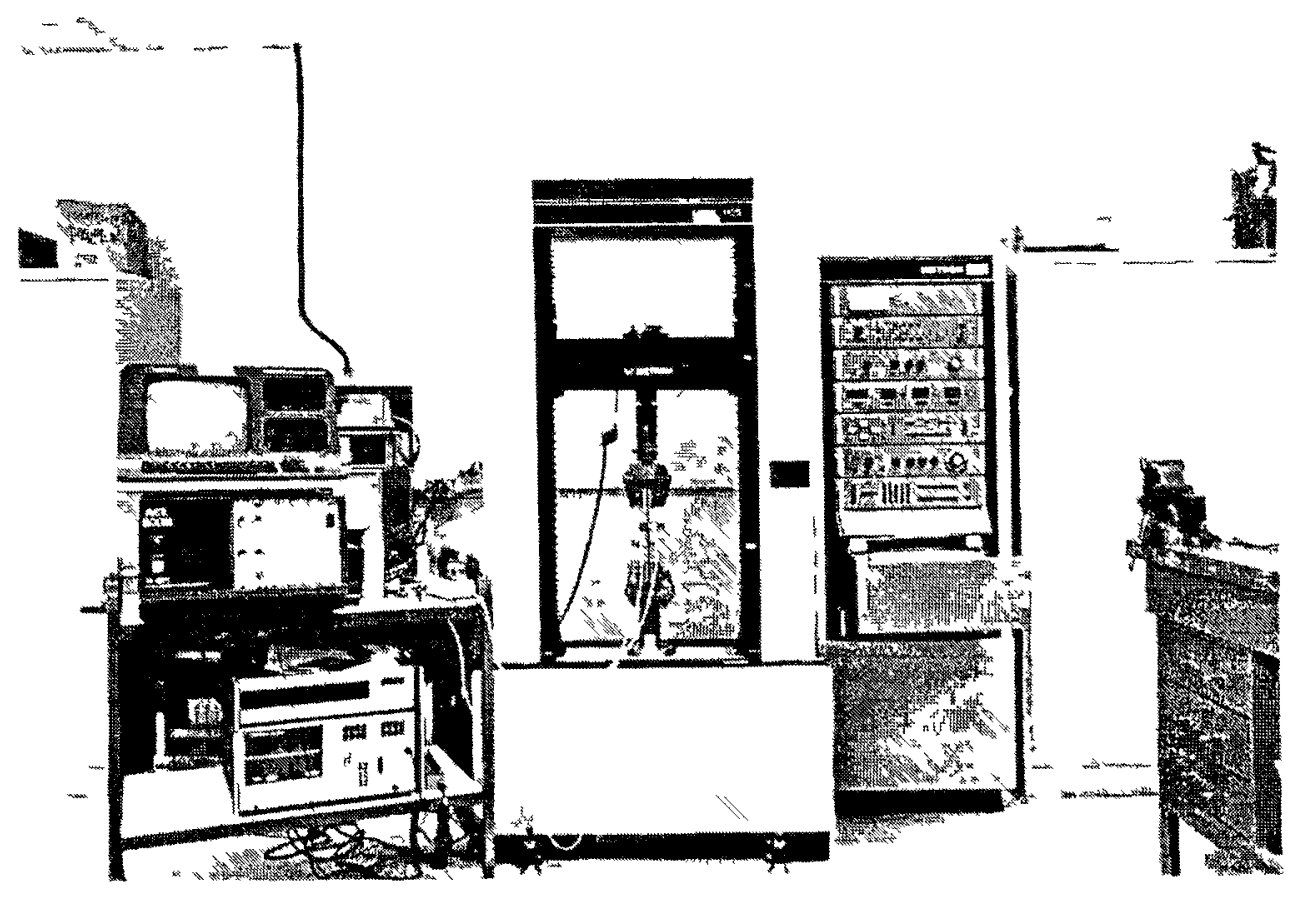

$\begin{aligned} & \text { Figure } 8.2: \text { Apparatus for measuring acousto-ultrasonic } \\ & \text { parameter (AUP) during the tensile loading of } \\ & \text { a joint specimen }\end{aligned}$ 


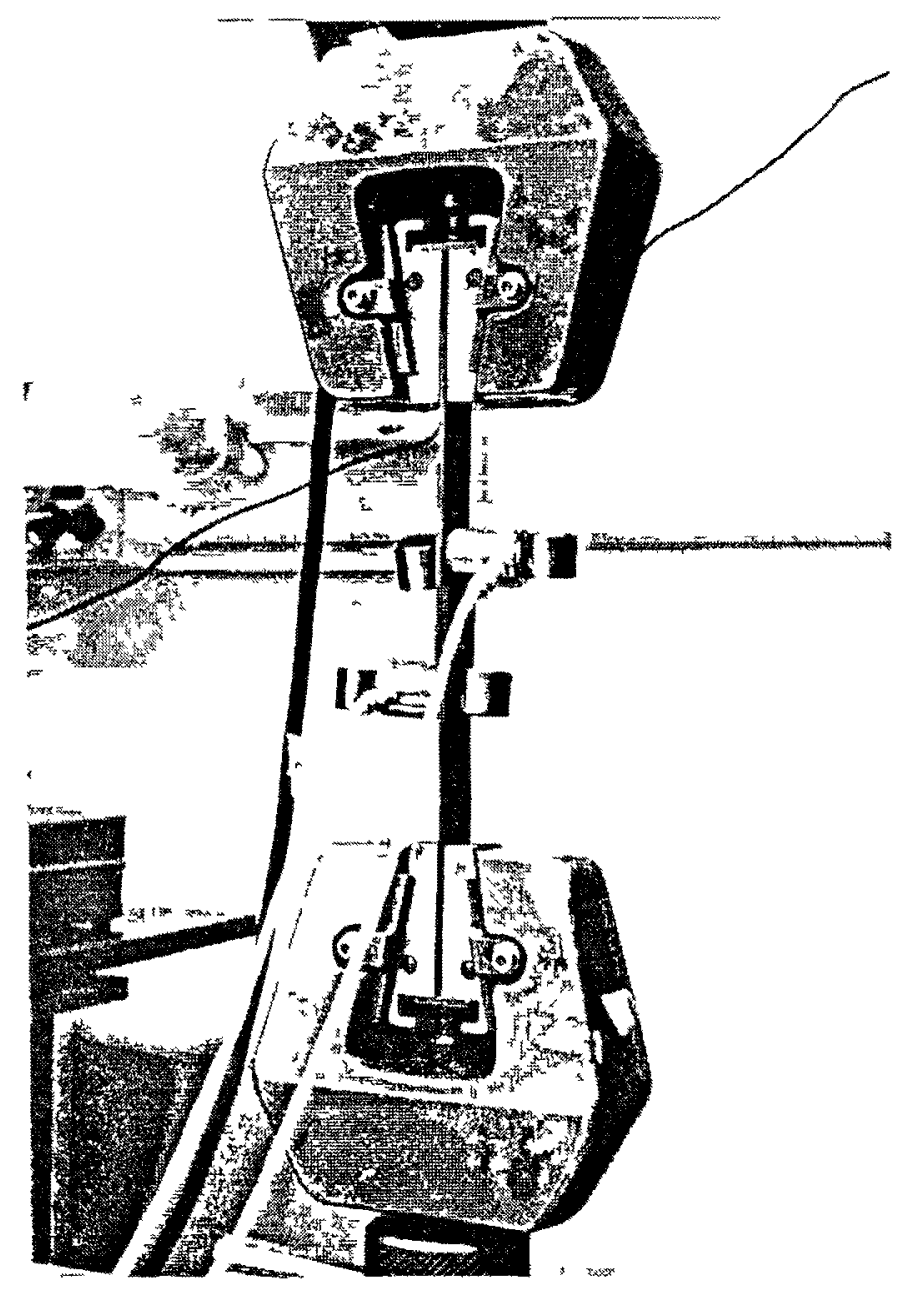

Figure 8.3 : Constant force clamps are used to hold the transducers to the specimen with Ultragel II as the coupling fluid 


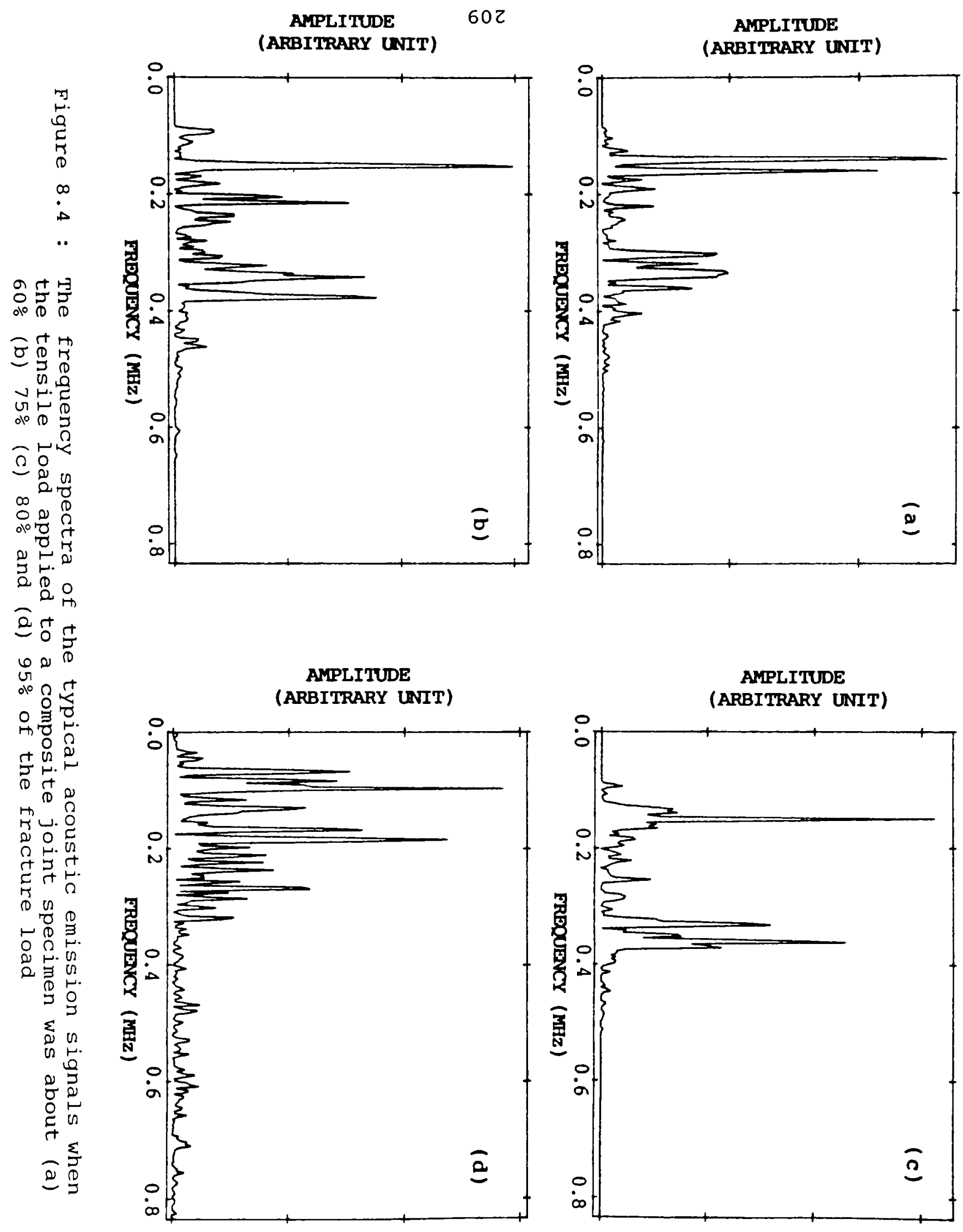




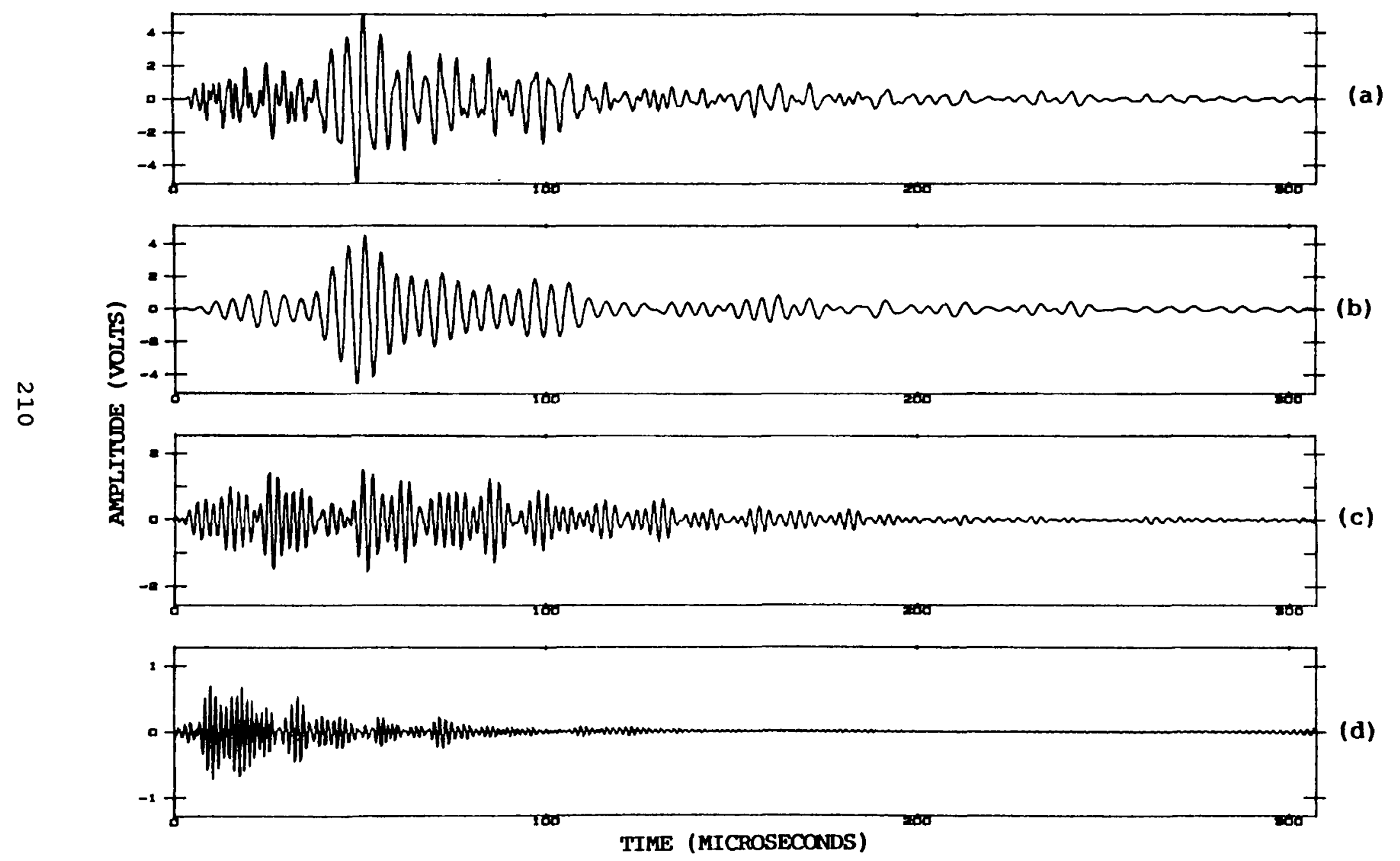

Figure 8.5: Typical AU waveforms with frequency content in the range of (a) 0.0 to $1.7 \mathrm{MHz}$ (b) 0.0 to $0.275 \mathrm{MHz}$ (c) 0.275 to $0.550 \mathrm{MHz}$ and (d) 0.550 to $0.875 \mathrm{MHz}$ for a representative bonded joint in composites 


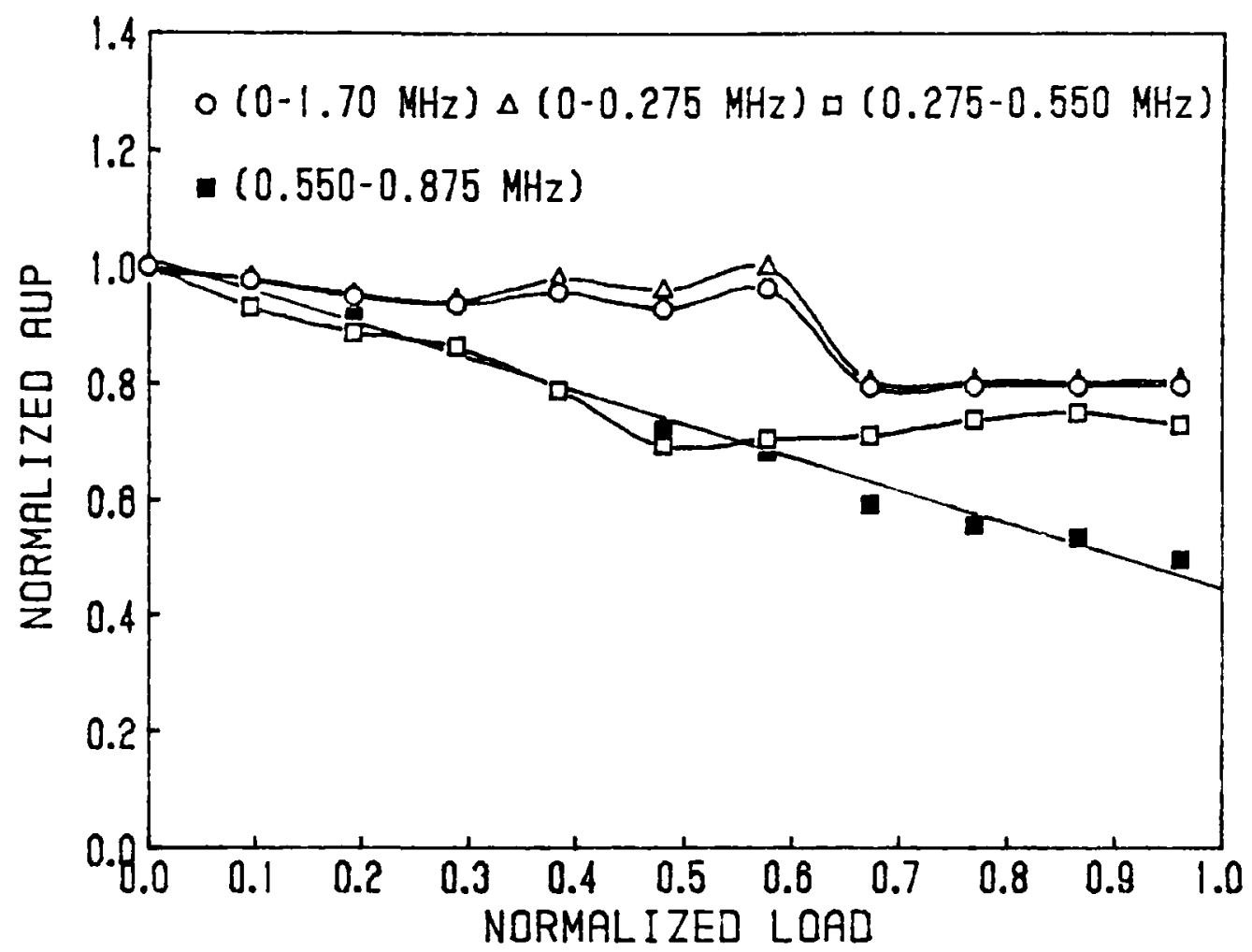

Figure 8.6 : The normalized AUP of the waveforms with four different ranges of frequency content vs the normalized load for a representative composite specimen 


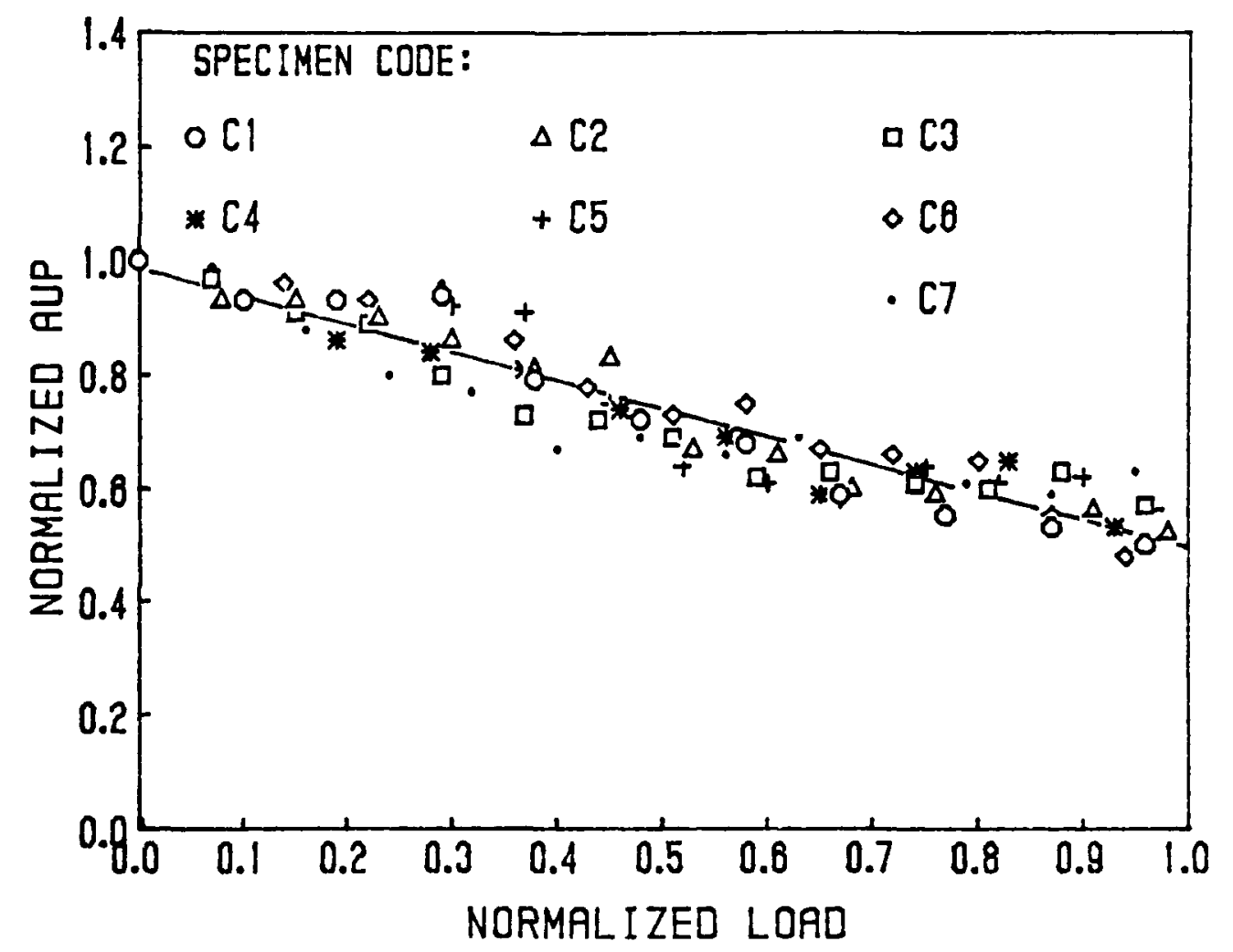

Figure 8.7 : The normalized AUP of the waveforms with frequency content in the range 0.550 to 0.875 $\mathrm{MHz}$ vs the normalized load for composite specimens. The solid line represents the best fit to the data points using the least squares method 


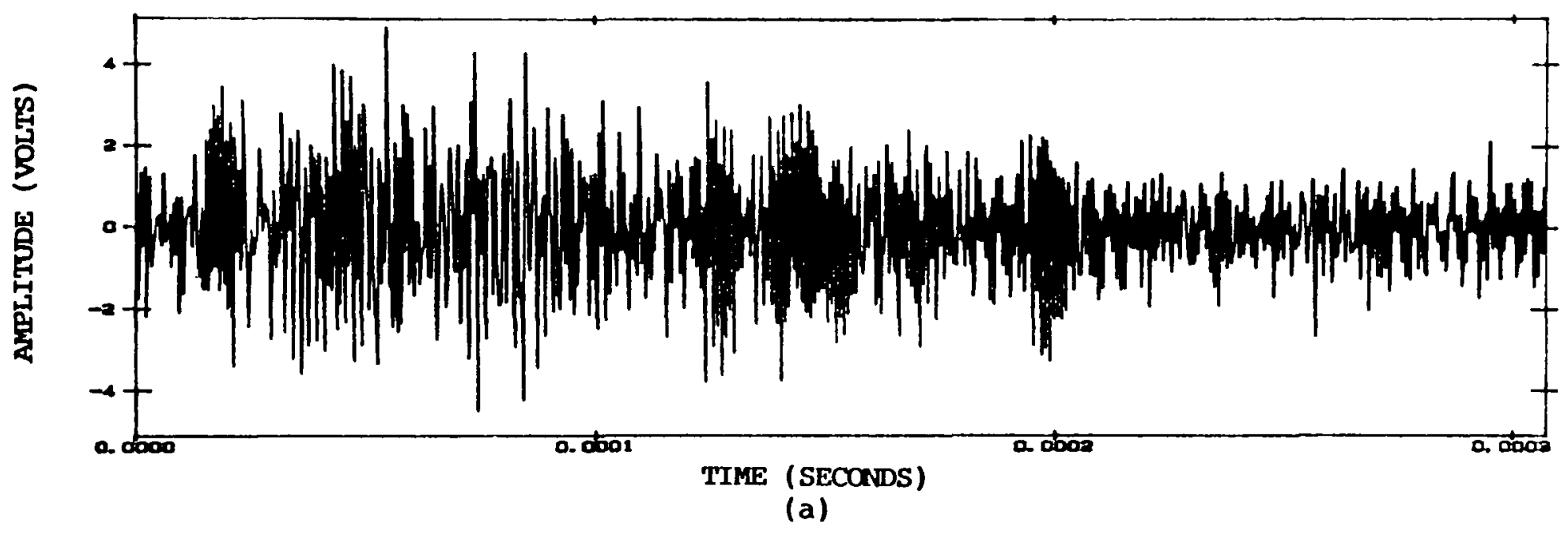

$\stackrel{\sim}{\omega}$

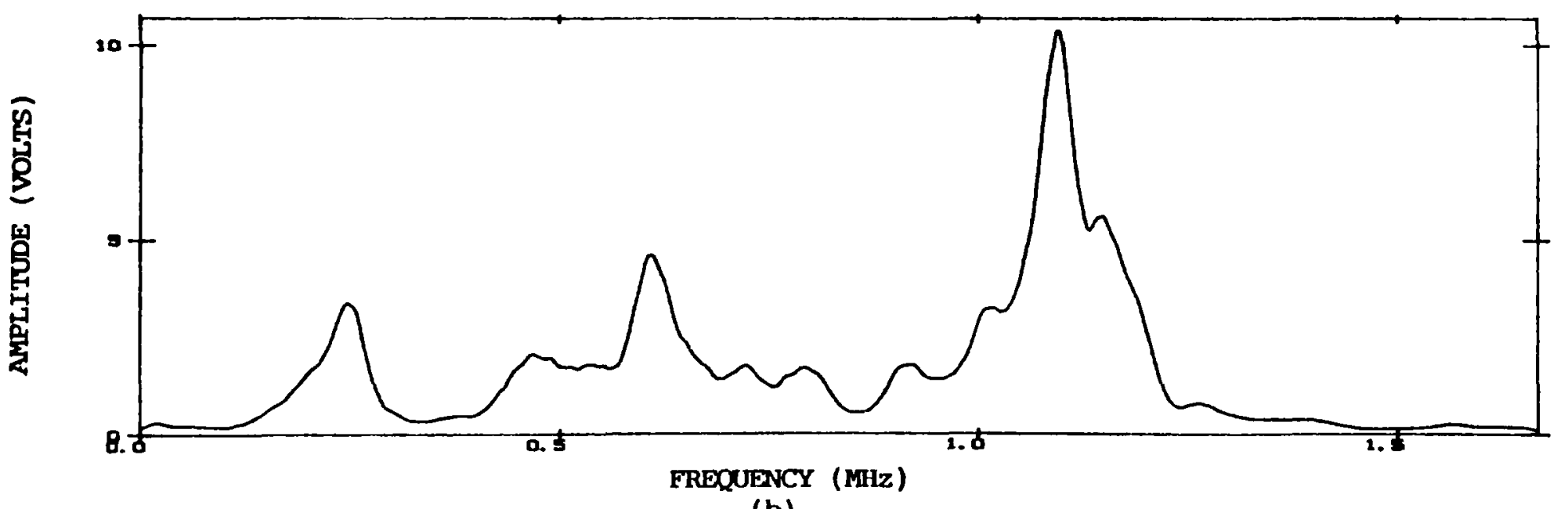

(b)

Figure 8.8: A typical acousto-ultrasonic waveform in (a) time domain and (b) frequency domain signals for a representative bonded joint specimen in $n+\infty 01$ 

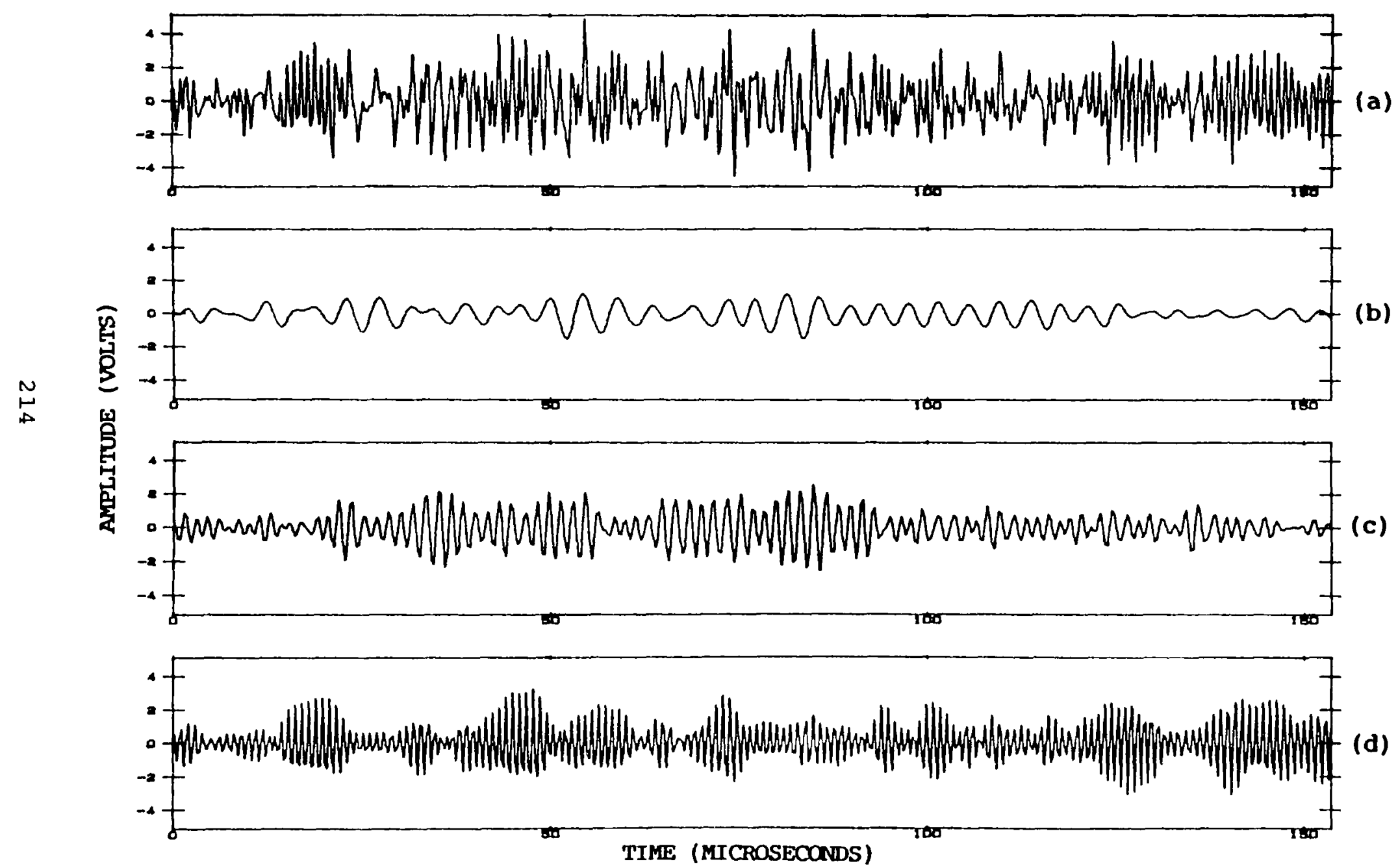

Figure 8.9 : Typical acousto-ultrasonic waveforms with frequency content in the range of (a) 0.0 to $1.7 \mathrm{MHz}$ (b) 0.0 to $0.4 \mathrm{MHz}$ (c) 0.4 to $0.8 \mathrm{MHz}$ and (d) 0.8 to $1.2 \mathrm{MHz}$ for a representative steel bonded joint specimen 


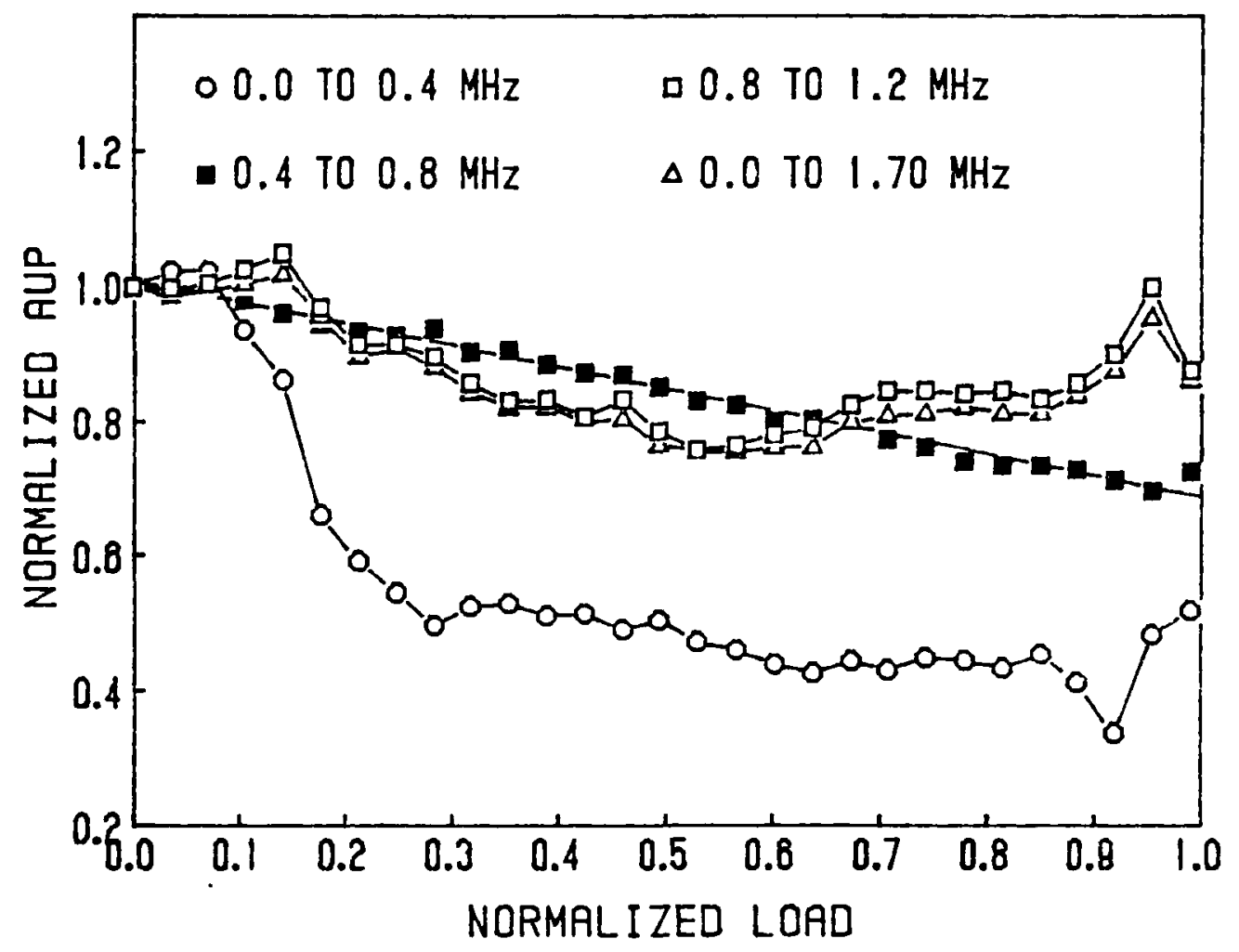

Figure 8.10 : The normalized AUP of the waveforms with four different ranges of frequency content vs the normalized load for a representative steel specimen 


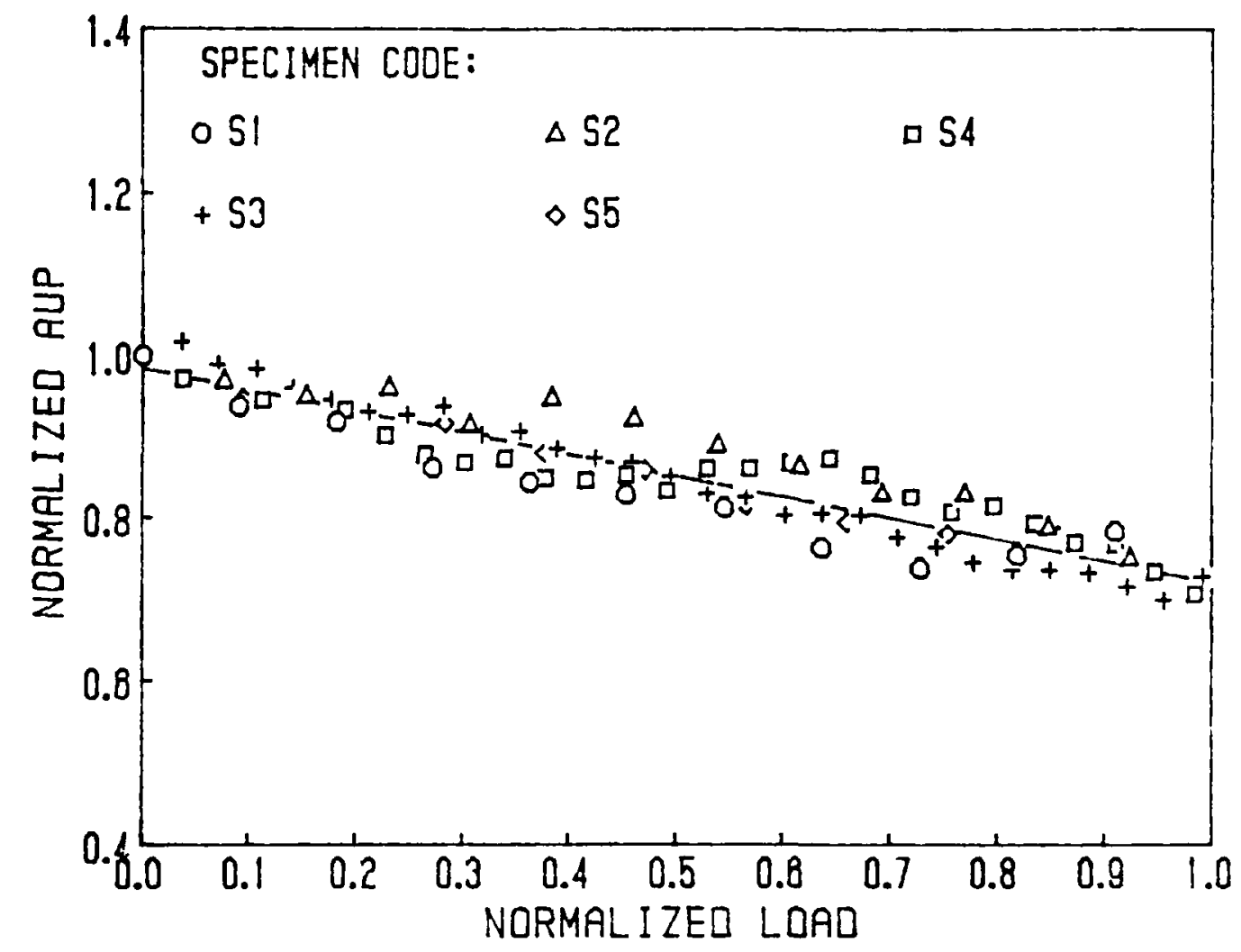

Figure 8.11 : The normalized AUP of the waveforms with frequency content in the range of 0.4 to 0.8 $\mathrm{MHz}$ vs the normalized load for steel specimens. The solid line represents the best fit to the data points using the least squares method 